\title{
Experimental and Analytical
}

\section{Research on the}

\section{Aerodynamics of Wind Turbines}

FINAL REPORT

December, 1977

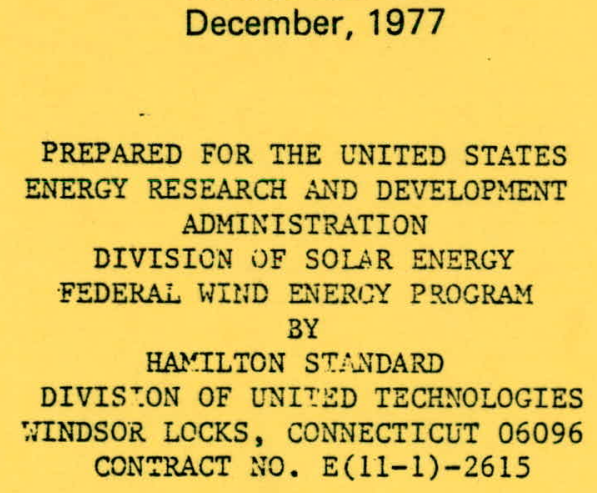

HAMHTON $3 y / 1 / /$ Division of

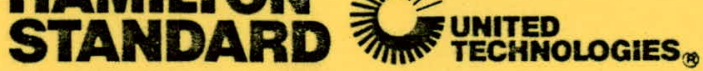




\section{DISCLAIMER}

This report was prepared as an account of work sponsored by an agency of the United States Government. Neither the United States Government nor any agency Thereof, nor any of their employees, makes any warranty, express or implied, or assumes any legal liability or responsibility for the accuracy, completeness, or usefulness of any information, apparatus, product, or process disclosed, or represents that its use would not infringe privately owned rights. Reference herein to any specific commercial product, process, or service by trade name, trademark, manufacturer, or otherwise does not necessarily constitute or imply its endorsement, recommendation, or favoring by the United States Government or any agency thereof. The views and opinions of authors expressed herein do not necessarily state or reflect those of the United States Government or any agency thereof. 


\section{DISCLAIMER}

Portions of this document may be illegible in electronic image products. Images are produced from the best available original document. 


\section{NOTICE}

This report was prepared as an account of work sponsored by an agency of the United States Government. Neither the United States nor any agency thereof, nor any of their employees, makes any warranty, expressed or implied, or assumes any legal liability or responsibility for any third party's use or the results of such use of any information, apparatus, product or process disclosed in this report, or represents that its use by such third party would not infringe privately owned rights. 
EXPERIMENTAL AND ANALYTICAL RESEARCH ON THE

AERODYNAMICS OF WIND DRIVEN TURBINES

FINAL REPORT

C. Rohrbach

H. Wainauski

R. Worobe1

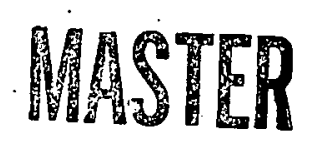

Hamilton Standard

Division of United Technologies Corporation

Windsor Locks, Connecticut 06096

December 1977

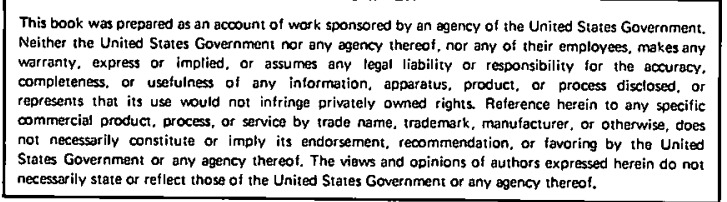

Prepared for the United States

Energy Research and Development

Administration

Division of Solar Energy

Federal Wind Energy Program

ERDA CONTRACT NO. E(11-1)-2615 
$\underline{\text { Section }}$

Page No.

EXECUTIVE SUMMARY . . . . . . . . . . . . . . .

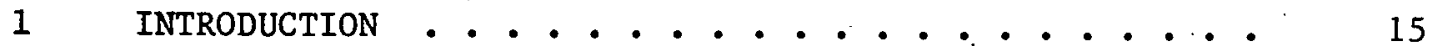

2 PROGRAM DESCRIPTION . . . . . . . . . . 16

2.1 Program Goals .............. 16

2.2 Program Outline ................. 16

3 AERODYNAMIC DESIGN AND PERFORMANCE METHODOLOGY... . . 20

3.1 Introduction . . . . . . . . . . 20

3.2 Goldstein Propeller Method ......... . 20

3.3 Rotor Vortex Wake Methods . . . . . . . . . 22

3.4 Skewed Wake Program ............. 24

3.5 Prescribed Wake Program . . . . . . . . . 24

3.6 Rotor Wake Geometry Program ......... 25

4 WIND TURBINE PARAMETKIC PERFORMANCE STUDY . . . . . . 31

4.1 Introduction . . . . . . . . . . . 31

4.2 Aerodynamics of Wind Turbines ........ . 31

4.3 Wind Turbine Parametric Performance Study . . . 34

5 LITERATURE SEARCH FOR EXPERIMENTAL DATA . . . . . . 79

6 SELECTION OF MODEL WIND TURBINE CONFIGURATIONS . . . 81

7. STRUCTURAL DESIGN OF WIND TURBINE MODELS . . . . . 89

7.1 Philosophy ................... 89

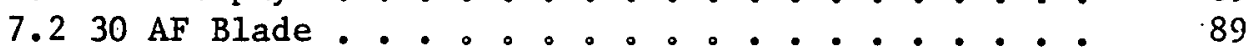

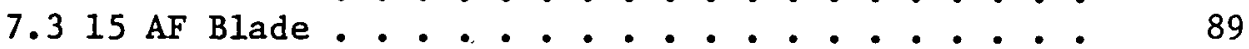

7.460 AF Blade ................... 93

8. MANUFACTURE OF MODELS . . . . . . . . . . 108

9 PREDICTED PERFORMANCE OF THE SELECTED WIND

TURBINE CONFIGURATIONS ................ 109

9.1 Predicted Aerodynamićs Performance ....... 109

9.2 Effect of Inflow on Performance ....... 110

9.3 Effect of Reynolds Number on the Performance of the 2 Blade/30 Activity Factor Model . . . . 110

9.4 Effect of Geometric Variables on Predicted Performance ................. 111

9.5 Effect of Surface Roughness on Performance . . . 111

10 WIND TUNNEL TEST PROGRAM . . . . . . . . 151

10.1 Introduction . . . . . . . . ... 151

10.2 Wind Tunnel Test Facility ........... 151 
10.3 Data Reduction and Accuracy . . . . . . 156

10.4 Experimental Data Package . . . . . . . 158

10.5 Supplemental Test Data .......... 161

11 DATA ANALYSIS AND EVALUATION . . . . . . . 198

11.1 Introduction . . . . . . . . . 198

11.2 Method Validation ............ 198

11.3 Effect of Prime Parameters . . . . . . . 201

11.4 Summary ................ 203

12. IDENTIFICATION OF AERODYNAMIC TECHNOLOGY NEEDS . . • 218

12.1 Introduction . . . . . . . . . . . 218

12.2 Continuation of Model Wind Turbine Test Program 218

12.3 Wind Turbine Performance Computer Program . . 219

12.4 Wind Turbine Airfoil Data . . . . . . . 220

12.5 Environmental Studies ........... 220

13 CONCLUSIONS . . . . . . . . . . . 221

14 REFERENCES ......................... 223

15 ACKNOWLEDGEMENTS . . . . . . . . . . 225

16 SYMBOLS . . . . . . . . . . . . 226

$\begin{array}{ll}\text { APPENDIXES } & 228\end{array}$

A - Original Test Schedule . . . . . . . . . 228

$B$ - Investigation of the Sepsration of a Blade From a Wind Turbine Model During Wind Tunnel Testing . . . . . . . . . . . 228

C - Data Reduction Equations . . . . . . . . 244 
1 Effect of Blade Shape Parameters on Annual. Energy Output ..$\quad 5$ 4-1. Effect of Blade Shape Parameters on Annual Energy Output . . 4 42 10-1 Run Schedule . . . . . . . . . . . . . . . . . . 163 10-2 Statement of Accuracies . . . . . . . . . . . . . . . 164 10-3 Effect of Velocity Measurement Accuracy on Power Ratio . . . 165 10-4 Effect of Power Measurement Accuracy on Power Ratio . . . . 165 10-5 Effect of Thrust Measurement Accuracy on Thrust Ratio • . . . 165 


\section{LIST OF FIGURES}

1 Variation of Power Ratio with Reynolds Number . . . . . . 6

Wind Turbine Rotor Performance for Optimum Blade Designs . . Blade Shape ........................ Blade Characteristics and Planform . . . . . . . . 30AF Model No. 2 in 5.48 Meter (18 Foot) Tunnel . . . . . Effect of Blade Angle Variation on Measured Power Ratio . . Comparison Between Measured and Predicted Power Ratio : . . Comparison. Between Measured and Predicted Power Ratio . . Comparison of the Effect of Different Methodologies on

Performance ..................... Comparison of Thrust Ratio Calculations and Experimental Data

4-1 Effect of Number of Blades on Peak Performance of Optimum Wind Turbines ...................

4-2 Effect of L/D on Peak Performance of Optimum 2-Bladed Wind

4-3 Effect of Velocity Ratio on Activity Factor of Optimum Wind Turbines .......................

4-4 Airfoil Section Lift-to-Drag Ratio Trend With Operating Lift Coefficient . . . . . . . . . . . . . . ..

4-5 Constraint of Activity Factor on Performance Spectrum of Optimum Wind Turbines ..................

4-6 Constraint of Performance and Activity Factor Limits on Performance Spectrum of Optimum 2-Bladed Wind Turbines . . .

4-7 Blade Planforms ...................

4-8 Thickness Distribution.................

4-9 Twist Distribution .................

4-10 Effect of Activity Factor on the Power Output of Near Optimum 2-Bladed Wind Turbines.................

4-11 Typical Velocity Duration Curves for Various Mean Wind Speeds

4-12 Kilowatts Versus Hours of the Year . . . . . . . . . .

4-13 Effect of Activity Factor on the Annual Energy Output of $57.9 \mathrm{M}$ (190 Foot) Diameter 2-Bladed Wind Turbines . . . . . . .

4-14 Effect of Number of Blades on the Power Output of Near Optimum

4-15 Effect of Number of Blades on the Annual Energy Output of 57.9M (190 Foot) Diameter 2-Bladed Wind Turbines...... Width Variations ..................

4-17 Effect of Planform Variations ..............

4-18 Effect of P.lanform on the Annual Energy Output of 57.9M (190 Foot) Diameter 2-Bladed Wind Turbines . . . . . . .

4-19 Thickness Variations ................

4-20 Effect of Thickness Distribution ...............

4-21 Effect of Thickness Ratio on the Annual Energy Output of 57.9M (190 Foot) Diameter 2-Bladed Wind Turbines ......

4-22 Twist Variations .................. 
4-25 Variation of Lift/Drag Ratio With Lift Coefficient....

4-26 Effect of Airfoil Section ................

4-27 Effect of Airfoil Sections on the Annual Energy Output of a 57.9M (190 Foot) Diameter Wind Turbine . . . . . . . . .

4-28 Effect of Reynolds Number on the Performance of the Basic Wind Turbine......... . . . . . . . . . . .

4-29 Effect of Reynolds Number on the Annual Energy Output of a 57.9M (190 Foot) Diameter Wind Turbine . . . . . . . .

4-30 Effect of Reynolds Number and Roughness on the Annual Energy Output of a 57.9M (190 Foot) Diameter Wind Turbine . . . .

4-31 Reynolds Number for Peak Power Ratio for an 8 Foot Diameter Wind Turbine. . . . . . . . . . . . . . . .

4-32 Roughness Effect on Power Ratio .............

4-33 Effect of Roughness on Annual Energy Output of a 57.9M (190 Foot) Diameter Wind Turbine ..............

4-34 Blade Root Cut-off ..................

4-35 Effect of Blade Root Cut-off ................

4-36 Effect on Blade Root Cut-Off on Annual Energy Output of a 57.9M (190 Foot) Diameter Wind Turbine . . . . . . .

6-1 ERDA Wind Turbine Characteristics, 15AF . . . . . . .

6-2 ERDA Wind Turbine Characteristics, 30AF ........

6-3 ERDA Wind Turbine Characterist1cs, 60AF . . . . . . .

6-4 Wind Turbine Rotor Performance for Optimum Blade Designs . .

6-5 Reynolds Number for Peak Power Ratio for an 8 Foot Diameter Wind Turbine....................

7-1 . Stress-Strength Comparison . . . . . . . . . . . .

7-2 Design Revisions of Thickness Distribution . . . . . . .

Critical Speed Diagram - 30AF . . . . . . . . . .

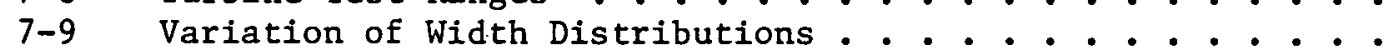

7-10 Blade and Spar Stresses ................

7-11 Critical Speed Diagram, 60AF ..............

7-12 Blade Airfoil Stacking, 15AF, 30AF . . . . . . . . .

7-13 Blade Airfoil stacking, 60AF ..............

9-1 Effect of Reynolds Number and Roughness on Annual Energy Output of a 57.9M (190 Foot) Diameter Wind Turbine . . . .

9-2A Blade Characteristics and Planform, 15AF . ........

9-2B Blade Shape ........ . . . . . . . . . .

9-2C Effect of Blade Angle and Mean Velocity Variation on Predicted Power Ratio ... . . . . . . . . . . . .

9-2D Effect of Blade Angle and Mean Velocity on Predicted Thrust Ratio . . . . . . . . . . . . . . . . .

9-3A Blade Characteristics and Planform, 30AF, 2 Blades.....

9-3B Blade Shape .....................

9-3C Effect of Blade Angle and Mean Velocity Variation on Predicted Power Ratio

9-3D Effect of Blade Angle and Mean Velocity Variation on Predicted 
9-4A Bläde Characteristics and Planform, 30AF, 3 Blades.....

9-4B Blade Shape .....................

9-4C Effect of Blade Angle and Mean Velocity Variation on Predicted Power Ratio . . . . . . . . . . . . . . . . . '

9-4D Effect of Blade Angle and Mean Velocity Variation on Predicted Thrust Ratio . . . . . . . . . . . . . . . . Blade Characteristics and Planform, 30AF, 1 Blade .... Blade Shape . . . . . . . . . . . . . . . . Effect of Blade Angle and Mean Velocity Variation on Predicted Power Ratio ..................... Predicted

9-5D Effect of Blade Angle and Mean Velocity Variation on Predicted Thrust Ratio . . . . . . . . . . . . . . . . Blade Characteristics and Planform, 60AF, 230XX Airfoils . Blade Shape . . . . . . . . . . . . . . . . . Effect of Blade Angle and Mean Velocity Variation on Predicted Power Ratio . . . . . . . . . . . . . . . . . Effect of Blade Angle and Mean Velocity Variation on Predicted Thrust Ratio . . . . . . . . . . . . . . . . Blade Characteristics and Planform, 60AF, 44XX Airfoils... Blade Shape . . . . . . . . . . . . . . . . . Effect of Blade Angle Variation and Mean Velocity on Predicted Power Ratio . . . . . . . . . . . . . . . . . .

9-7D Effect of Blade Angle Variation and Mean Velocity on Predicted Thrust Ratio . . . . . . . . . . . . . . . . . Wind Turbine Forces and Moments . . . . . . . . . . Effect of Inflow on Predicted Power Ratio .......... Effect of Inflow on Predicted Thrust Ratio . . . . . . . Effect of Inflow on Predicted Inplane Horizontal Force Ratio. Effect of Inflow on Predicted Inplane Vertical Force Ratio. • Effect of Inflow on Predicted Horizontal Plane Moment Ratio . Effect of Inflow on Predicted Vertical Plane Moment Ratio. . Effect of Reynolds Number on Predicted Power Ratio ..... Reynolds Number Nomograph . . . . . . . . . . . 
10-6D Effect of Blade Angle Variation on Measured Power Ratio, 30AF, 2 Blades ................... 180

10-6E Effect of Blade Angle Variation on Measured Thrust Ratio, 30AF, 2 Blades ...................... 181

$10-7 \mathrm{~A}$ 30AF Model No. 3 in 5.48 Meter (18 Foot) Tunnel ....... Effect of Repeat Runs on Measured Power Ratio, $8 \mathrm{~m} / \mathrm{s}$ (26 fps) Effect of Repeat Runs on Measured Thrust Ratio, $8 \mathrm{~m} / \mathrm{s}$ ( $26 \mathrm{fps}$ ) Effect of Repeat Runs on Measured Power Ratio, $14 \mathrm{~m} / \mathrm{s}$ (40 fps) Effect of Repeat Runs on Measured Thrust Ratio, $14 \mathrm{~m} / \mathrm{s}$. (46 fps) Effect of Repeat Runs on Measured Power Ratio, $22 \mathrm{~m} / \mathrm{s}$ (73 fps) Effect of Repeat Runs on Measured Thrust Ratio, $22 \mathrm{~m} / \mathrm{s}$ (73 fps) Effect of Velocity on Measured Power Ratio . . . . . . . Effect of Velocity on Measured Thrust Ratio . . . . . . . Effect of Blade Angle Variation on Measured Power Ratio . . Effect of Blade Angle Variation on Measured Thrust Ratio . .

11-1A Comparison Between Measured and Predicted Power Ratio, 15AF,

11-1B Comparison Between Measured and Predicted Thrust Ratio, $15 \dot{1} \dot{A}$, 2 Blades . . . . . . . . . . . . . . . . . .

11-2A Comparison Between Measured and Predicted Power Ratio, 30AF, 2 Blades . . . . . . . . . . . . . . . . . . .

11-2B Comparison Between Measured and Predicted Thrust Ratio, 30AF, 2 Blades . . . . . . . . . . . . . . . . . . "

11-3A Comparison Between Measured and Predicted Power Ratio, 30AF, 3 Blades . . . . . . . . . . . . . . . . .

11-3B Comparison Between Measured and Predicted Thrust Ratio, 30AF, 3 Blades . . . . . . . . . . . . . . . . .

11-4 Comparison Between Measured and Predicted Power Ratio, All Models ...... . . . . . . . . . . . . . . Comparison Between Measured Model and Predicted Full Scale.

11-6 Comparison of the Effect of Different Methodologies on Performance . . . . . . . . . . . . . . . . . Effect of Airfoil Data on Performance . . . . . . . . . Measured Variation of Power Output with Reynolds Number . . 


\section{INTRODUCTION}

The successful development of reliable, cost competitive horizontal axis, propeller-type wind energy conversion systems (WECS) is strongly dependent on the availability of advanced technology for.each of the system components. Past experience and current studies of this type of wind energy conversion system have shown that the wind turbine subsystem most significantly affects the system's cost effectiveness and performance capability. Thus, adequate technology bases are essential for all elements of the wind turbine design. Accordingly, in recognition of this need, ERDA sponsored the analytical and experimental research program on wind turbine aerodynamics conducted by Hamilton Standard under ERDA Contract E(11-1)-2615 and reported herein.

This aerodynamic research program was aimed at providing a reliable, comprehensive data base on a series of wind turbine models covering a broad range of the prime aerodynamic and geometric variables. Such data obtained under controlled laboratory conditions on turbines designed by the same method, of the same size, and tested in the same wind tunnel had not been available in the literature. Moreover, this research program was further aimed at providing a basis for evaluating the adequacy of existing wind turbine aerodynamic design and performance methodology, for assessing the potential of recent advanced theories and for providing a basis for further method development and refinement.

In order to achieve these goals, the program reported herein included several tasks which are discussed in detail in the text. Although not all of the goals were accomplished because the program was abbreviated due to a testing accident, the work completed provides a comprehensive treatise of wind turbine aerodynamic design and performance characteristics for the practical range of operation. A summary of the work accomplished is presented below.

\section{PARAMETRIC STUDY}

The first part of this program was an analytical parametric study of the important wind turbine geometric parameters. In order to accomplish this study, a suitable aerodynamic design and performance prediction method needed to be selected. In this connection several rotor theories were reviewed and discussed. These programs included the Goldstein Propeller Method, the Prescribed Wake Program, the Rotor Wake Geometry Program and the Skewed Wake Program. Each of these programs was reviewed for immediate application to this study. In view of its mature status and many years in basic propeller design analysis, the Hamilton Standard Propeller Method (based on the Goldstein theory) was modified for energy extraction rather than energy input to the fluid medium and utilized as the basic analytical tool for this study and, later, for the aerodynamic design of the test wind turbine models. The method incorporates two-dimensional performance data on several airfoil families, including the effect of Reynolds number and surface roughness. Thus the method permits the thorough study of all geometric parameters as well as scale effects and blade surface conditions.

In addition to this method, another existing program, called the Transition Program, was modified and used for computing the performance of the wind turbine in inclined flow. Finally, the new Prescribed Wake and Free Wake 
Programs were modified for wind turbine application to permit their evaluation against test data.

The parametric study of the prime blade geometric parameters included activity factor, number of blades, planform, thickness and twist distributions, airfoil section, Reynolds number, blade root cut-off, and airfoil surface condition. The results of this study are summarized in Table 1. The effect of the parameters mentioned above on annual energy output is presented as percent change relative to the basic 2 blade $/ 57.9 \mathrm{~m}$ (190 ft) diameter/30 AF/trapezoidal planform/NACA $230 \mathrm{XX}$ airfoil wind turbine. The table indicates that only increasing number of blades and airfoil type resulted in an improvement in annual energy output over the reference wind turbine. Large deviations from the optimum blade shape in planform, thickness distribution, and twist distribution will result in significant losses. Root cut-off beyond $15 \%$ will also result in large losses. The model test data are expected to show lower performance than that predicted for full scale wind turbines because of limitations in test Reynolds number. Figure 1 presents a plot of power ratio at the design velocity ratio as a function of Reynolds number. Further, calculations indicate significant losses due to surface roughness and waviness.

Thus, on the basis of this parametric study, the blade shape parameters deemed most significant and which require experimental confirmation were established as activity factor, number of blades, airfoil section and surface condition.

MODEL WIND TURBINE TEST PROGRAM

The second part of this program covered the wind tunnel testing of $2.44 \mathrm{~m}$ ( $8 \mathrm{ft}$ ) diameter model wind turbines, the analysis of the measured performance, and the comparison with predicted performance. In pursuit of these objectives, six wind turbine models were designed and manufactured. As mentioned previously, the shape characteristics of the test models were selected based on the parametric study reviewed above. An optimum aerodynamic trapezoidal planform was incorporated in each of the models. The other important shape parameters for the six models are listed below.

\begin{tabular}{cc} 
MODEL & ACTIVITY FACTOR \\
\hline 1 & 15 \\
$2 *$ & 30 \\
3 & 30 \\
4 & 30 \\
5 & 60 \\
6 & 60
\end{tabular}

\begin{tabular}{c} 
NO. BLADES \\
\hline 2 \\
2 \\
3 \\
1 \\
2 \\
2
\end{tabular}

AIRFOIL SECTION

* REFERENCE MODEL

The predicted full scale peak power ratio of each model at the design velocity ratio is presented in Figure 2. A photograph of the reference model is shown in Figure 3 and the corresponding shape characteristics are shown in Figure 4. The other models are of the same family, differing in basic parameters as indicated in the above table.

A thorough mechanical design evaluation of each blade shape was made prior to manufacture of the blade. This evaluation considered such factors as strength, critical speed, and stall-flutter. Based on this evaluation, it was necessary to tailor the thickness ratio distribution of each blade design to avoid critical speed and strength problems. 
Each of the listed models was to be tested in the United Technologies Research Center's subsonic, $5.48 \mathrm{~m}$ (18 ft) diameter wind tunnel on a specially designed test rig. The test rig incorporated a wind turbine loading system and torque and rpm sensors. All other components of turbine force were measured on the main tunnel six-component balance. The rig was designed so that by varying the frequency of the loading motor, the turbine speed (rpm) could be controlled. Figure 5 presents a photograph of the reference model installed on the test rig in the wind tunnel.

Each model was tested over a range of tip speeds and tunnel velocities sufficient to completely define the performance from the velocity ratio of zero power output to that for stalled operation. Such data were obtained for several pitch settings representative of variable pitch capability. The data are presented in the familiar terms of power ratio ( $P_{R}$ ) and thrust (drag) ratio (TR) as a function of velocity ratio (VR) for a range of pitch settings. The primary performance data were automatically recorded and reduced on-line to allow continuous perusal of the results. In addition, all measurements were recorded off-line for later, reduction and analysis.

Unfortunately, testing was terminated due to a blade separation before all of the models had been tested. A discussion of the blade accident is covered in the main report. At the time of the separation only three of the models had been completely tested. These were: Model 1 (15 activity factor, two-bladed turbine), Model 2 (30 activity. factor, two-bladed turbine) and Model 3 (30 activity factor, three-bladed turbine) each incorporating the $230 \mathrm{xX}$ airfoil sections.

Excellent test results were obtained on each of the models. These results generally substantiate the predicted trends in performance with changes in solidity and number of blades. The performance map for the reference model is shown in Figure 6 as a plot of $P_{R}$ versus VR. Similar data on the other models together with the corresponding axial force data are presented in the main report. The data are considered to be reliable except at tunnel velocities below $10 \mathrm{~m} / \mathrm{sec}$ where small measurement errors cause large variations in power ratio because of the velocity cubed effect on this parameter.

The test results were compared with the predicted performance and generally showed good correlation in trends. Figure 7 compares the measured and predicted performance for the reference model 2. The plot shows the predicted performance based on model Reynolds number is similar but significantly lower in level compared to the corresponding test data. Similar relationships were observed for the other test models. It is believed that the relatively high turbulence level of the tunnel may have resulted in a turbulent boundary layer on the model. Thus the measurements may have simulated full scale Reynolds numbers. However, the unexpected sharp drop in performance as the velocity ratio is decreased below the peak where the model tip speed was reduced may be a Reynolds number effect. Thus for future tests, it is recommended that the models be run at constant tip speed, thereby holding Reynolds number essentially constant. Figure 8 shows that the comparative performance of the test models is essentially as predicted except for the levels, as mentioned previously. A study of Figure 9 indicates that several available aerodynamic methods utilizing airfoil data at model Reynolds number predict essentially the same performance for model 2, each showing power ratio levels below the test. Similar calculations utilizing airfoil data at full scale RN match test data in level but overestimate performance where the sharp fall-off occurs and at high velocity ratios near zero power ratio. This comparison tends to confirm the belief that the measured performance is representative of full. scale over the important operating range around peak power ratio. 
In conclusion, this wind turbine test program generally confirms the predicted performance trends with the prime blade shape parameters. Moreover, model wind turbine testing provides an accurate measure of full scale wind turbine performance provided that Reynolds numbers of at least $3.5 \times 10^{5}$ are achieved and that tunnel velocities can be within \pm .5 percent. 
TABLE 1

EFFECT OF BLADE SHAPE PARAMETERS ON ANNUAL ENERGY OUTPUT

Reference Wind Turbine: 2 Blades/57.9 m (190 ft.) Diameter/30AF/Trapezoidal Plan/NACA 230XX Airfoil.s

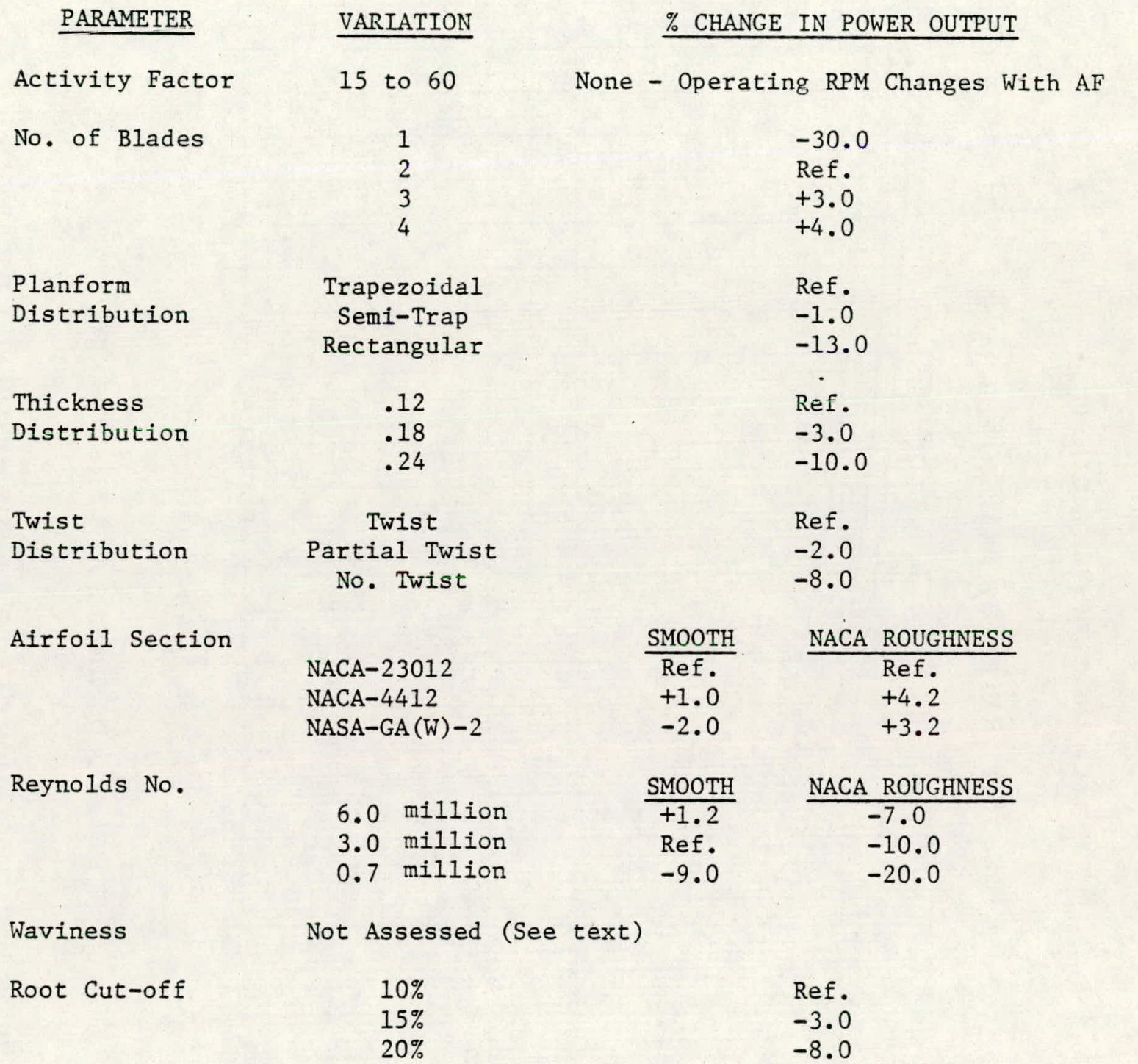


NACA 230XX AIRFOIL SECTIONS

2 BLADES

VELOCITY RATIO FOR PEAK POWER RATIO

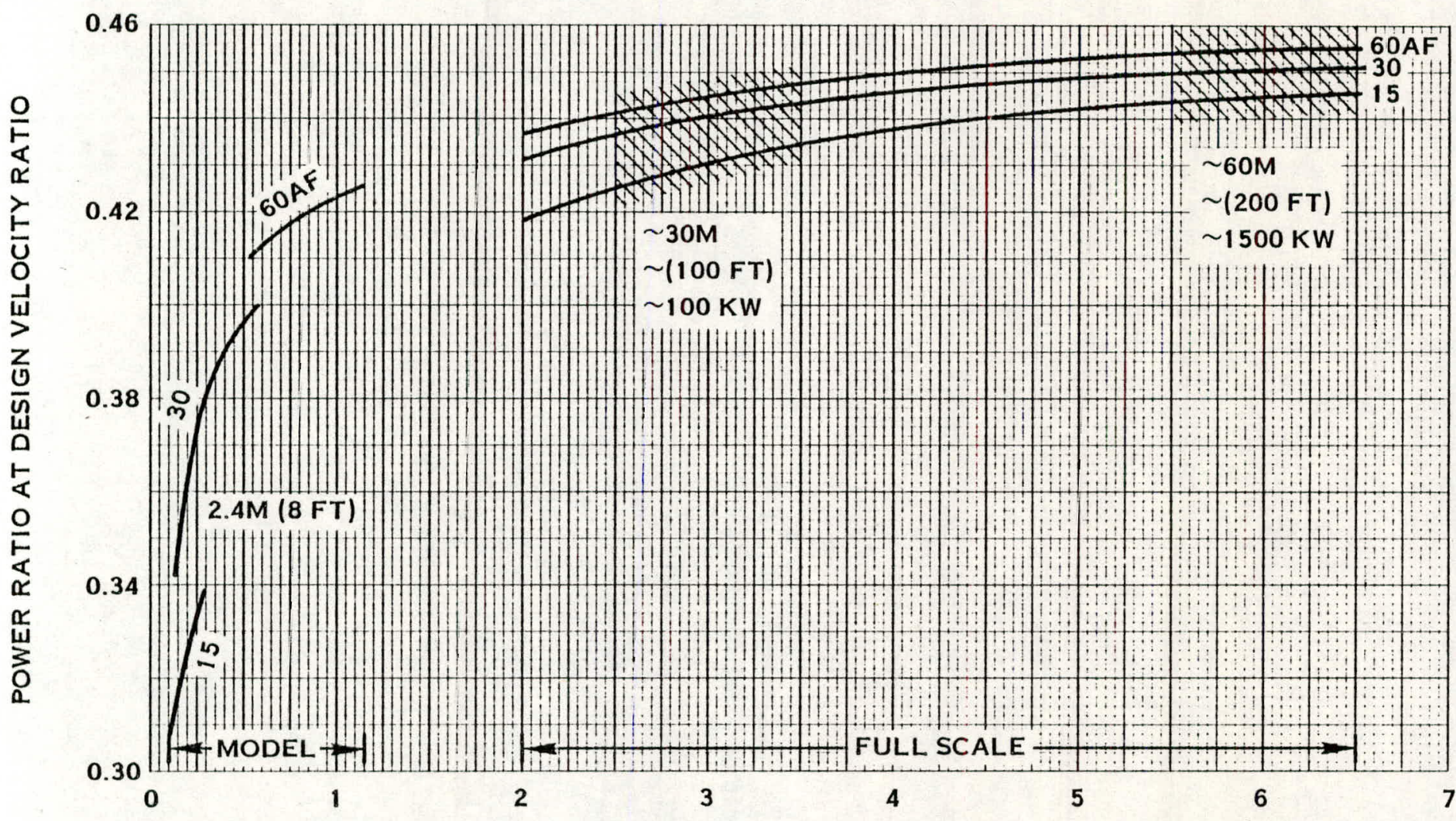

REYNOLDS NUMBER AT 3/4 RADIUS $\times 10^{-6}$

FIGURE 1. VARIATION OF POWER RATIO WITH REYNOLDS NUMBER 
MODELS NO. 2, 3, AND 4

30 ACTIVITY FACTOR

NACA $230 \times X$ AIRFOIL SECTIONS

$2.4 \mathrm{M}(8.0 \mathrm{FT})$ DIAMETER

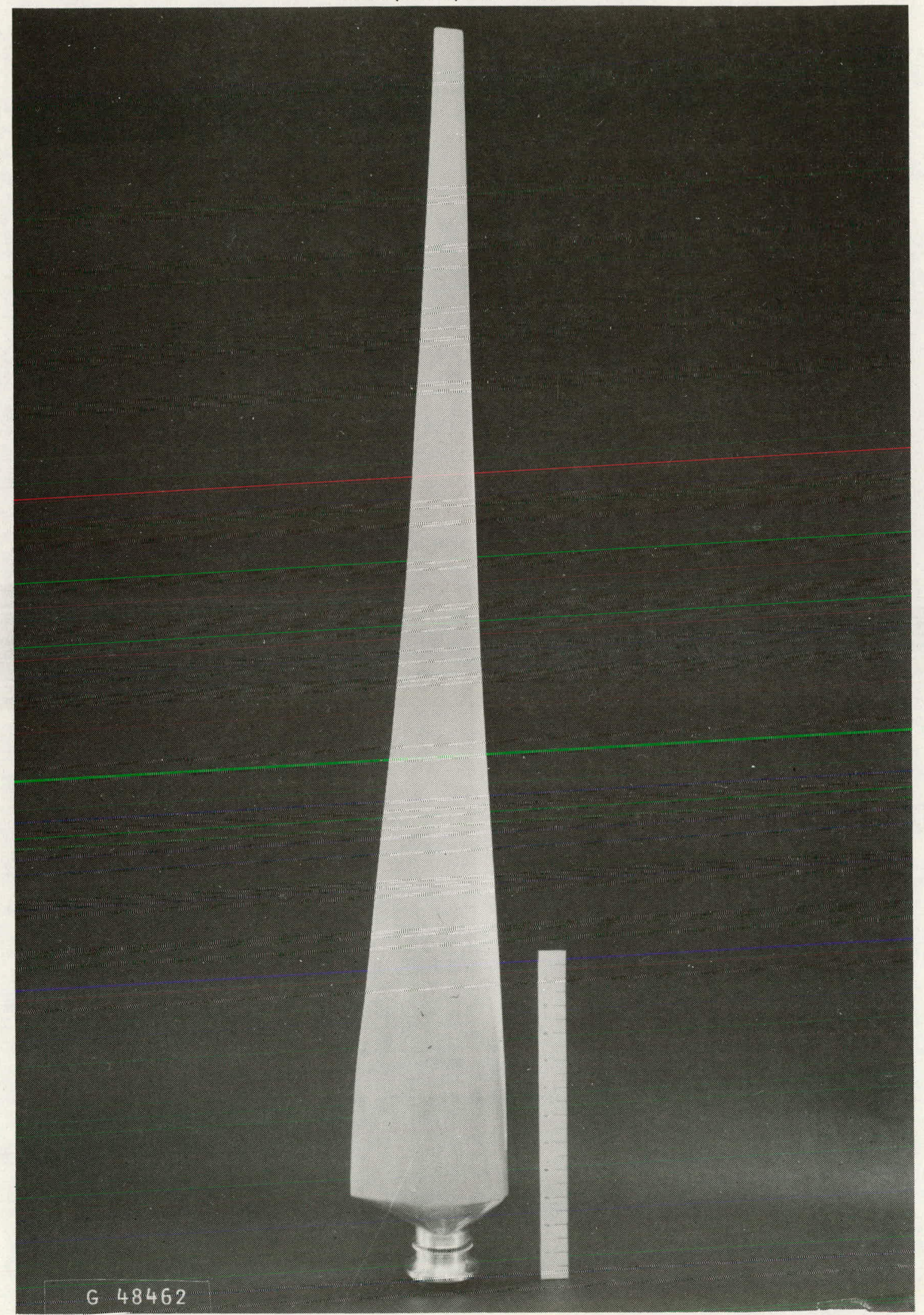

0

।

I

I

I

I

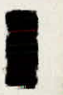

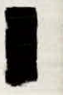

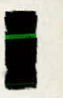

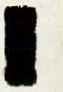

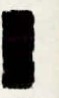

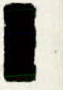

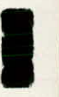

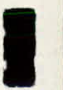

I

$\bullet$

FIGURE 3. BLADE SHAPE

$-8-$ 


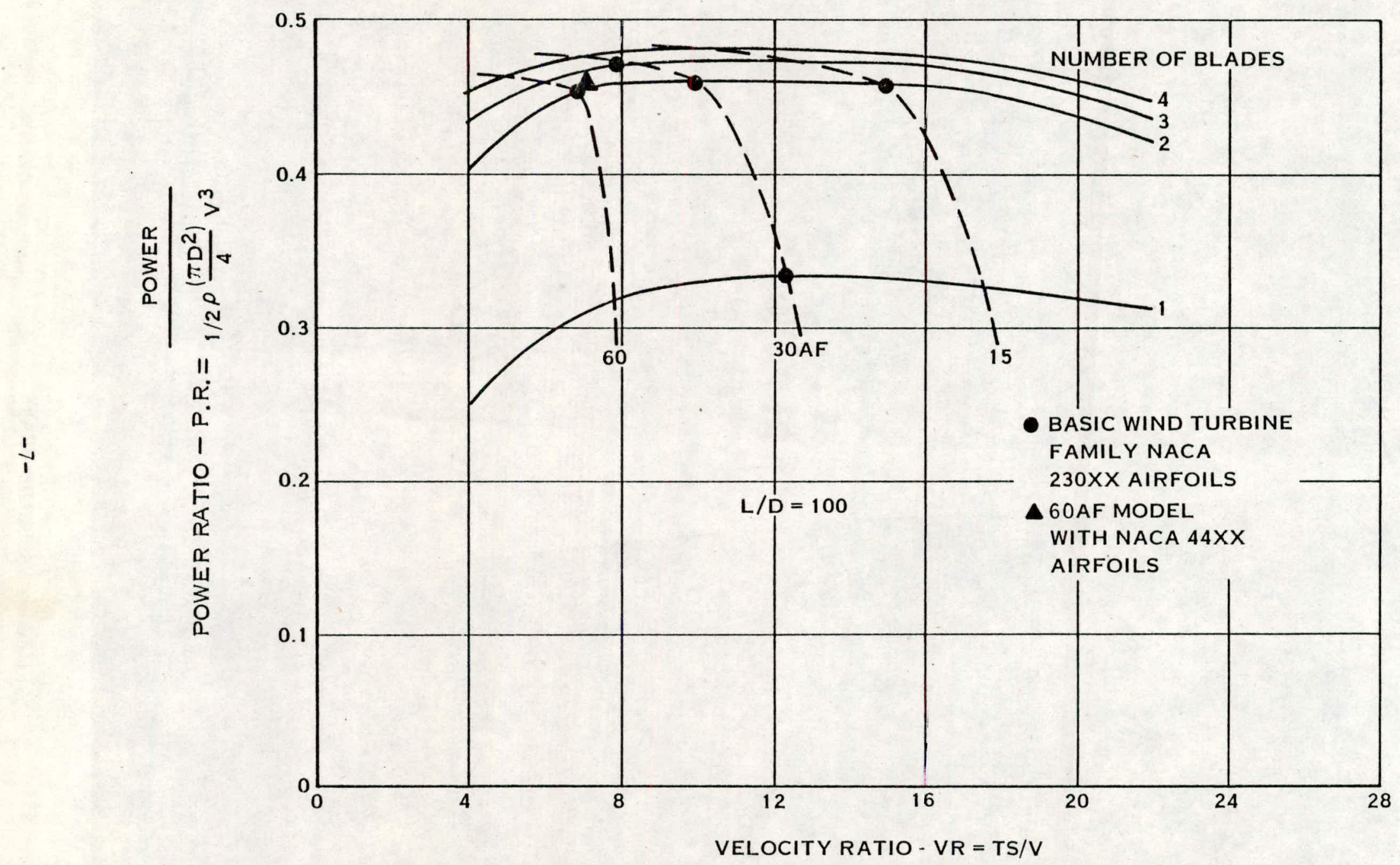

FIGURE 2. WIND TURBINE ROTOR PERFORMANCE FOR OPTIMUM BLADE DESIGNS 

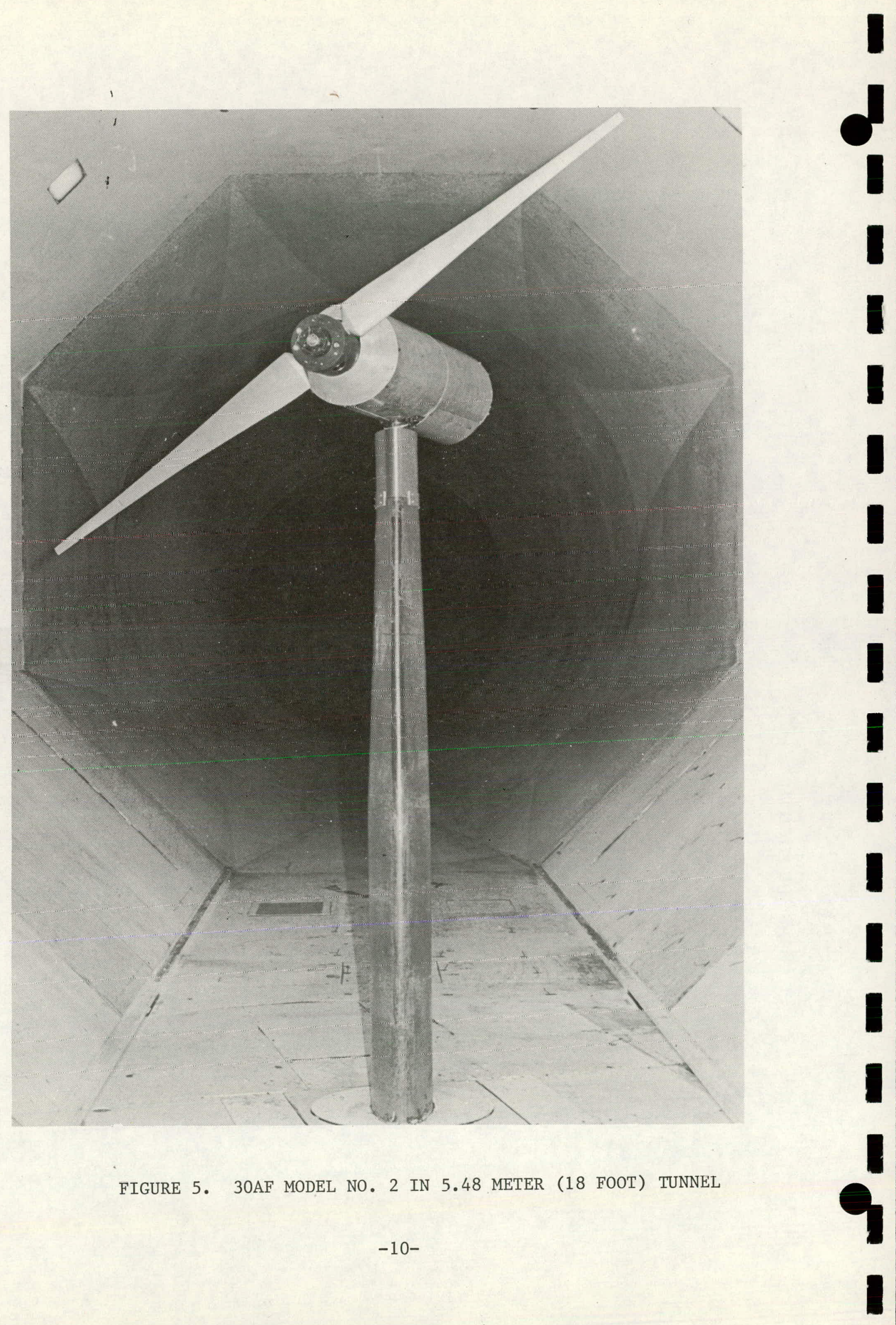

FIGURE 5. 3OAF MODEL NO. 2 IN 5.48 METER (18 FOOT) TUNNEL 
MODEL NO. 2 (REFERENCE MODEL)

\section{ACTIVITY FACTOR \\ NACA $230 X X$ AIRFOIL SECTIONS \\ 2.4 M (8.0 FT) DIAMETER \\ 2 BLADES}

BLADE PLANFORM

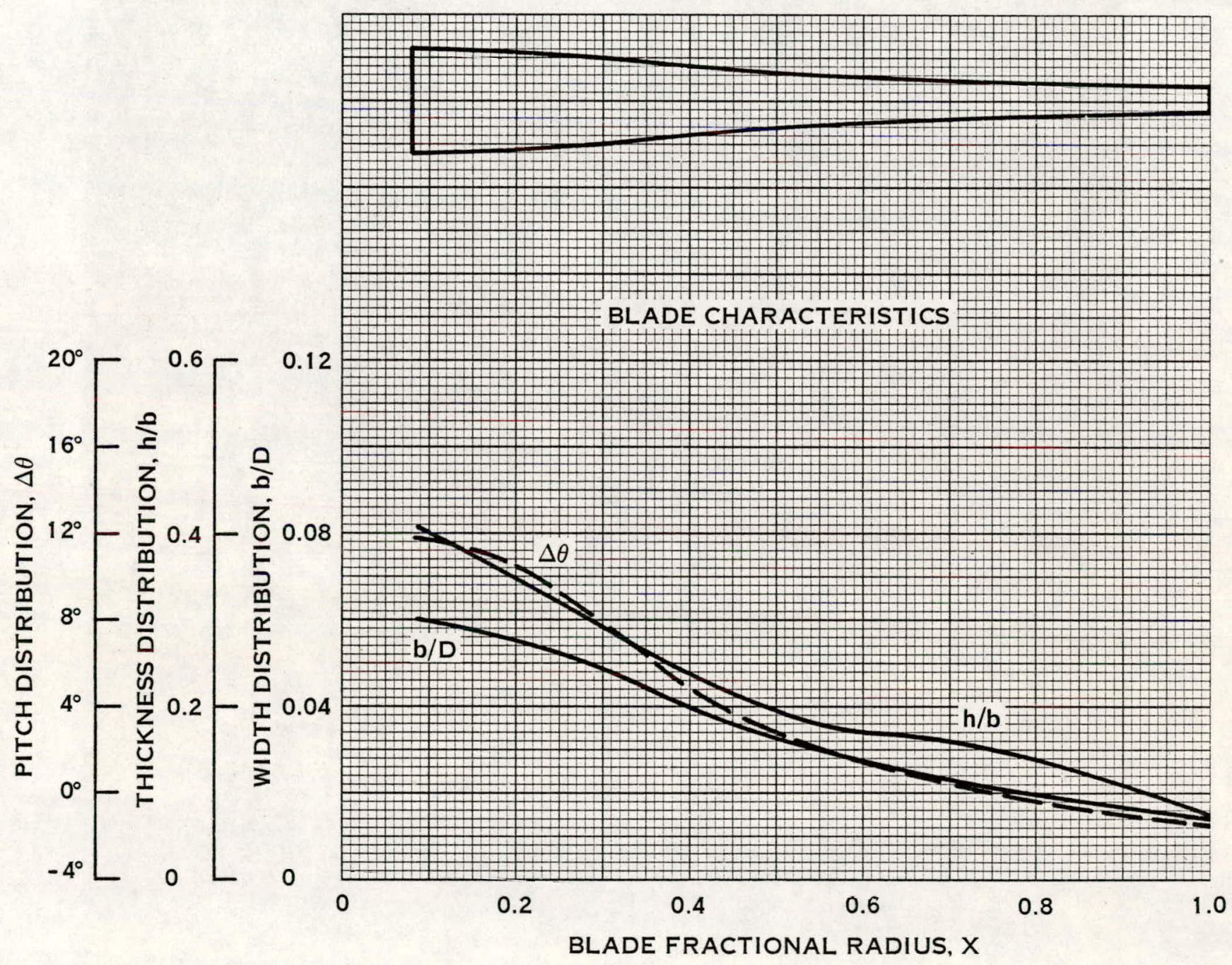

FIGURE 4. BLADE CHARACTERISTICS AND PLANFORM 
MODEL NO. 2

30 ACTIVITY FACTOR

NACA 230XX AIRFOIL SECTIONS

2 BLADES

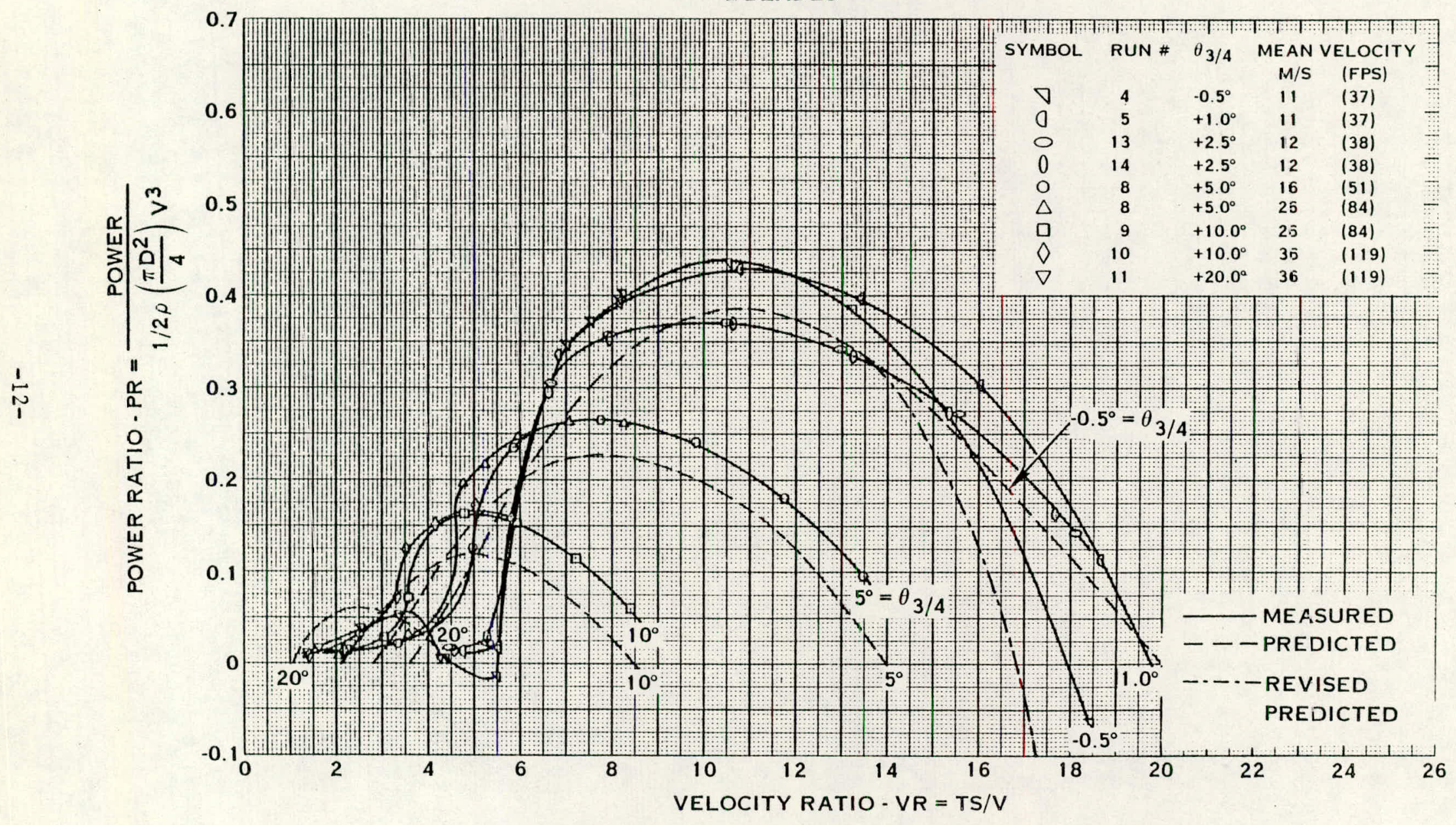

FIGURE 7. COMPARISON BETWEEN MEASURED AND PREDICTED POWER RATIO 
30 ACTIVITY FACTOR

NACA 230XX AIRFOIL SECTIONS

2 BLADES

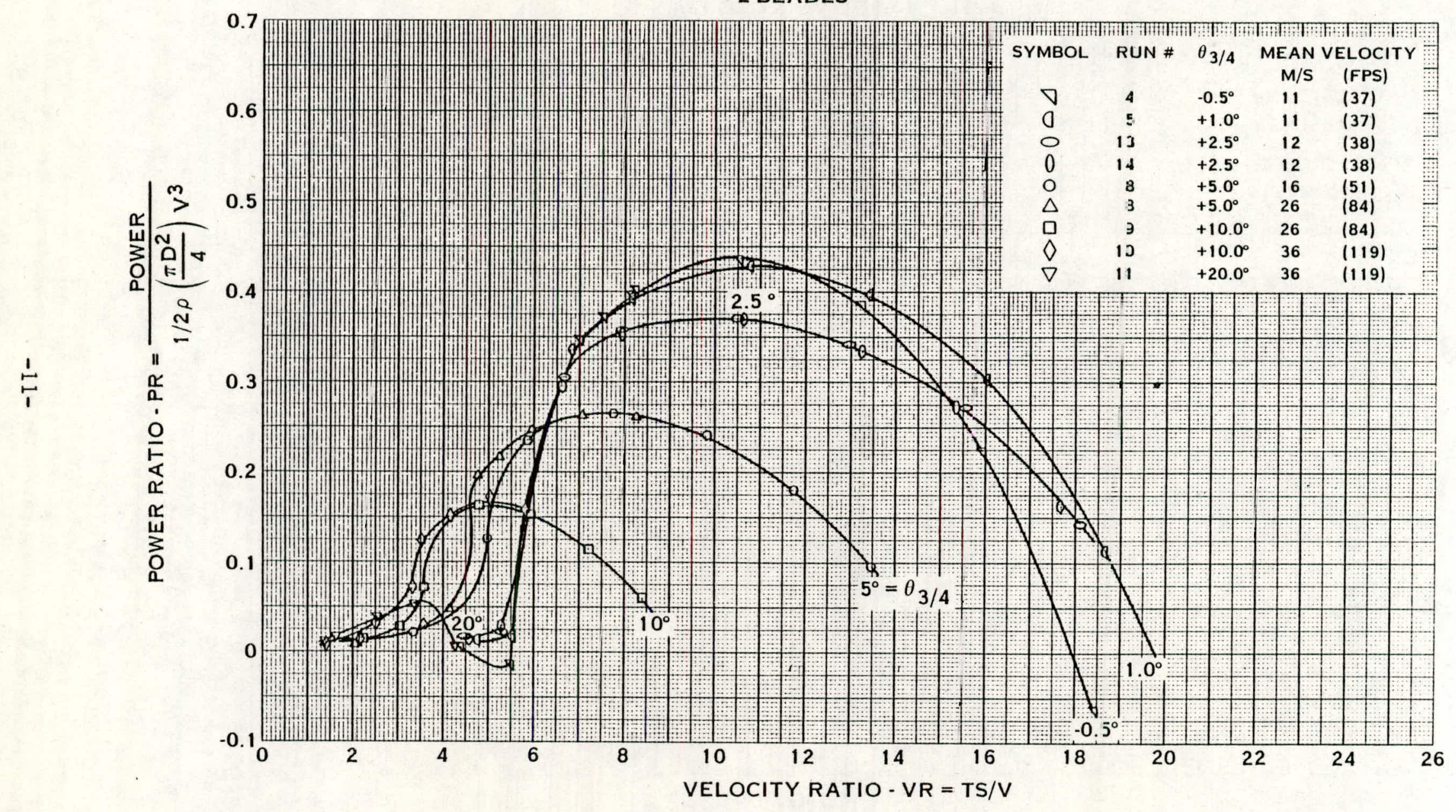

FIGURE 6. EFFECT OF BLADE ANGLE VARIATION ON MEASURED POWER RATIO 


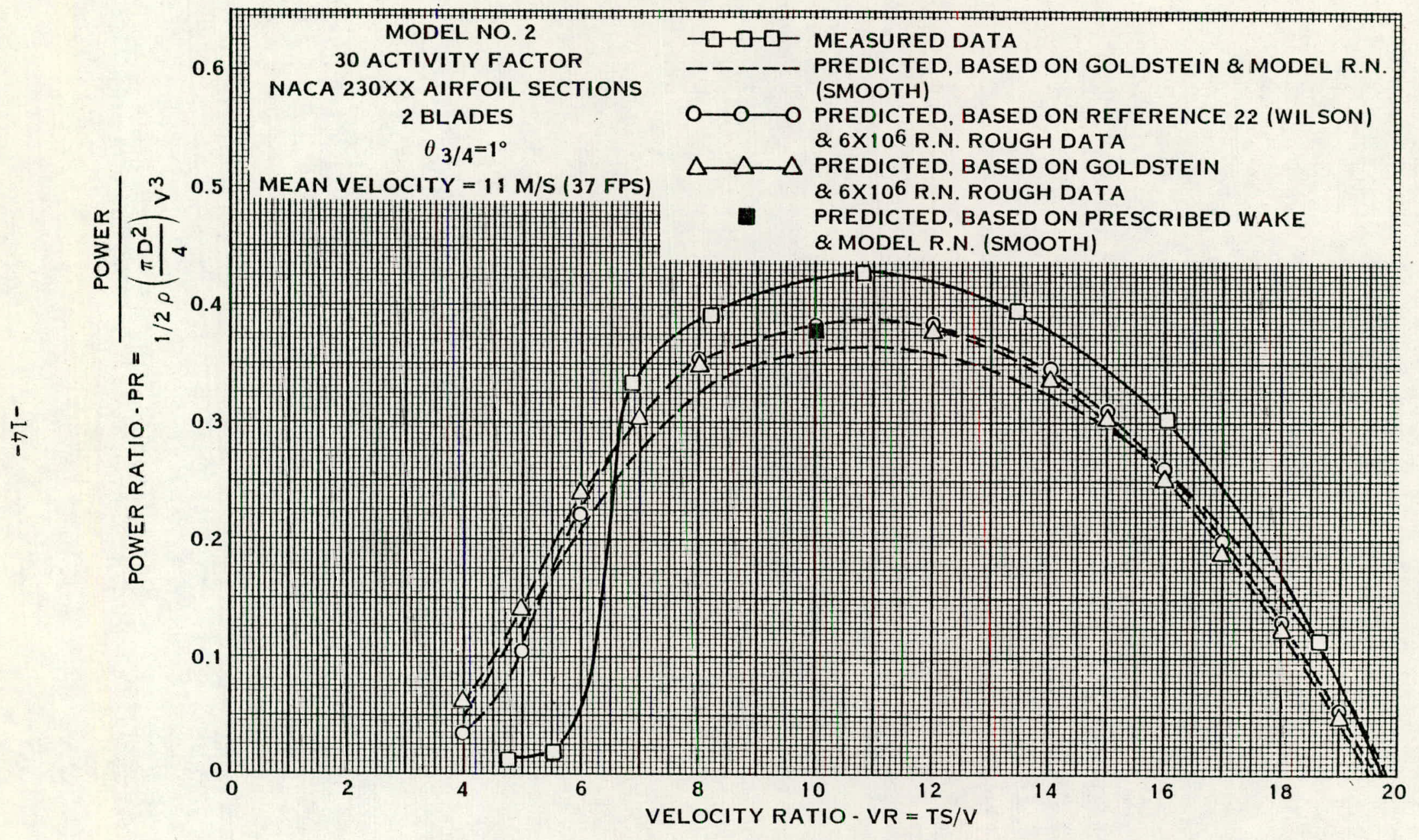

FIGURE 9. COMPARISON OF THE EFFECT OF DIFFERENT METHODOLOGIES ON PERFORMANCE 


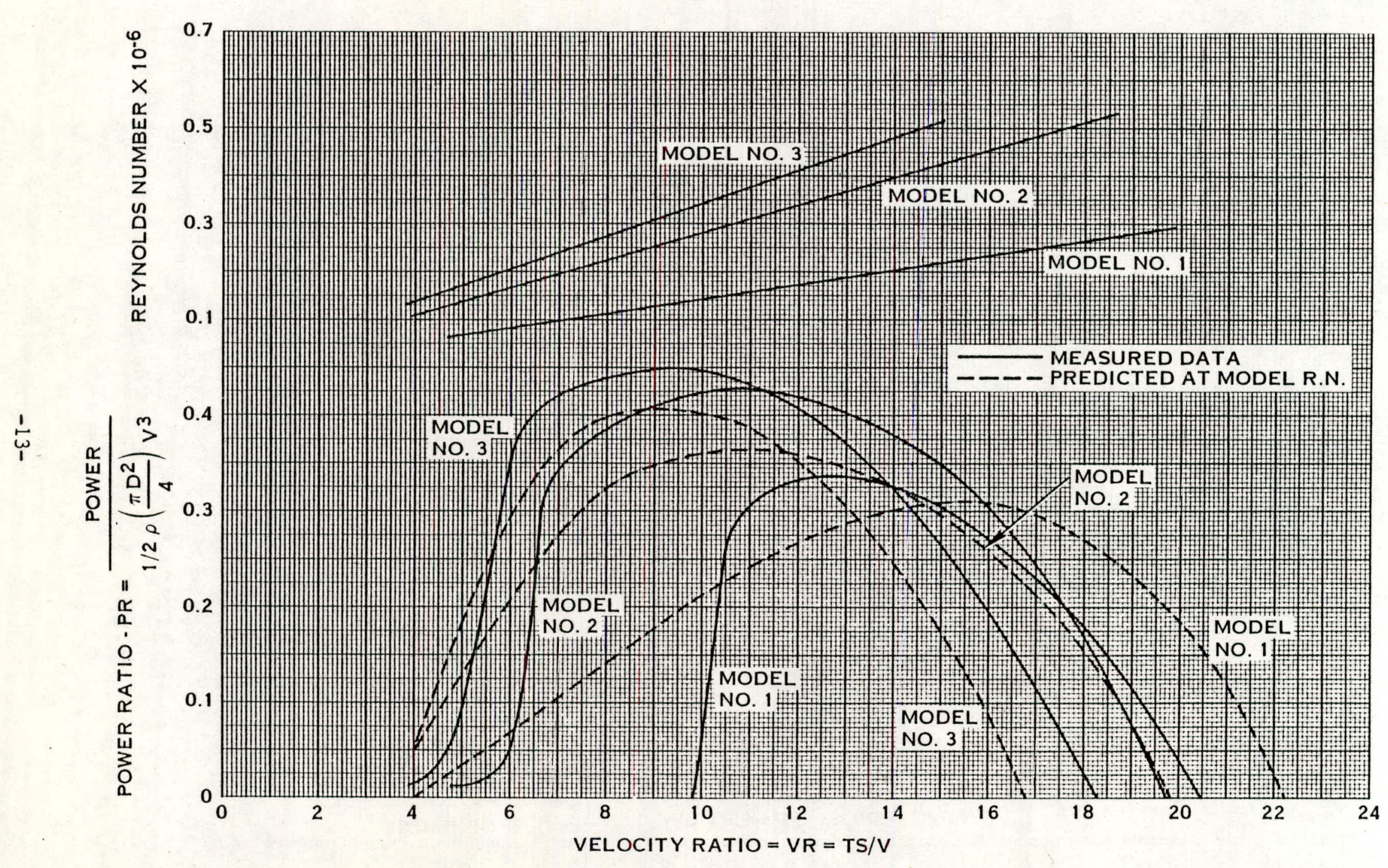

FIGURE 8. COMPARISON BETWEEN MEASURED AND PREDICTED POWER RATIO 


\section{SECTION 1}

\section{INTRODUCTION}

Wind energy conversion systems have long been utilized throughout the world to lighten man's burdens. All through history wind turbines have developed the power to mill grain, pump water and to generate electricity. These wind machines have ranged from the crude designs of ancient times and primitive people to the sophisticated configurations of the post World War II era. In the USA, wind turbine power generators reached maximum popularity and usage during the 1800's and early 1900's in farming areas throughout the country. However, with the coming of low cost electricity through rural electrification of the $1930^{\prime} \mathrm{s}$, the wind turbine virtually disappeared except in rare instances where they have been operated for useful work and as picturesque reminders of earlier times.

Recently, with the petroleum-fuel shortages in the industrialized countries of the world and in recognition of the limited petroleum reserves, renewed interest has been given to the wind turbine power generator as a possible source of energy to supplement fossil fuel and nuclear energy systems.

The successful development of reliable, cost competitive, horizontal axis, propeller-type wind energy conversion systems, (WECS), is strongly dependent on the availability of advanced technology for each of the system components. Past experience and recent studies of this type of WECS have shown that the wind turbine subsystem most significantly effects the systems cost effectiveness and performance capability. Thus adequate technology bases are essential for all aspects of the wind turbine design.

In this connection, proven aerodynamic design criteria for the wind turbine rotor are urgently needed. Accordingly, in recognition of this need the Energy Research and Development Administration, ERDA, has sponsored the experimental and analytical research programs on wind turbine aerodynamics conducted and reported herein by Hamilton Standard, Division of the United Technologies Corporation under ERDA Contract E(11-1)-2615.

Specifically, the analytical effort includes, (1) the conversion of existing propeller aerodynamic programs for the performance prediction and design of wind turbines, (2) the undertaking of a parametric study to investigate the effects of the prime aerodynamic and geometric wind turbine variables on performance, (3) on the basis of this study, the eelcction of a serles of wind turbine configurations for a wind tunnel test, (4) the conduct of a literature search to acquire additional data to supplement the test data obtained in the test phase and (5) the calculation of the complete performance of each test configuration to compare with the measured performance.

The experimental effort includes, (I) the design and manufacture of the test model wind turbines, (2) the planning and conducting of the wind tunnel test program, (3) the analysis of the test results and (4) the identification of additional needed research. 
SECTION 2

PROGRAM DESCRIPTION

\subsection{PROGRAM GOALS}

In undertaking to establish sound aerodynamic design criteria for wind turbine blading, this aerodynamic research program was aimed at providing a reliable, comprehensive data bank on a series of wind turbines covering a broad range of prime geometric and aerodynamic variables. Such data obtained under controlled laboratory conditions on model wind turbines designed by the same methods, of the same size and tested over the same operating conditions and Reynolds number range in the same wind tunnel had not been available in the literature. Furthermore, this research program was aimed at providing a basis for evaluating the adequacy of existing wind turbine aerodynamic design and ferformance prediction methodology and for refining them as required, for assessing the potential application of recent, advanced rotor theories, and for indicating areas requiring further research.

\subsection{PROGRAM OUTLINE}

The program included several specific tasks directed at accomplishing the goals set forth above. Each task is briefly described in the following text.

Task 1 - Aerodynamic Design and Performance Methodology

The initial task was to refine the existing Hamilton Standard computer programs ficr the aercdynamic design and performance prediction methods for wind turbines based on the widely used Goldstein propeller vortex theory and on a non-axial inflow method called the Skewed Vake Program developed within the United Technologies Corporation (UTC). In addition, two new advanced rotor performance methods based on more accurate definitions of the wake also developed within UTC were adapted for wind turbine application. The first of these new methods incorporates a wake shape defined by flow visualization data and the second method incorporates a free wake which is analytically defined. Unfortunately, as will be discussed later, the flow visualization wake data were nct obtalned as originally planned. However, that concept is reviewed in section 3. The free wake program is described and the predicted wind turbine performance compared to that predicted by the modified Goldstein theory.

For the aerodynamic design of the test wind turbine models, existing Hamilton Standard propeller programs were adapted for wind turbine application as discussed in detail later into the text. Moreover, additional two-dimensional (2-D) data on several airfoil families sultable for wind turbines were added to the existing airfoil data bank.

To analyze conditions where the wind direction is inclined to the turbine plane; i.e. during wind shift and tower turning, the inflow program mentioned above was adapted for application to wind turbines. The required modifications to allow for energy extraction effects on the wake shape and position were derived and the computer frogram revised accordingly. Firally the new Prescribed Wake and Free Wake Programs were rodified for wind turbine application and checked out. 
Task 2 - Wind Turbine Parametric Performance Study

In the second task, a wind turbine parametric performance study was conducted to investigate the effects of aerodynamic and geometric vind turbine variables on performance. Aerodynamically optimum turbines were considered as well as effects of off-optimum geometric variations of thickness distributions, planform shape, twist distributions and hub-totip ratio on performance. Variation of blade number, solidity, airfoil type, Reynolds number and blade surface conditions of performance were investigated. This study incorporated the envelope of operating conditions consistent with acceptable performance and practical, cost effective blade structures. The resulting data are presented in terms of output power coefficient versus the ratio of turbine tip speed to wind velocity. These parametric performance data provided a basis for selecting the wind turbine configurations included in this wind tunnel progran.

Task 3 - Literature Search for Experimental Data

In support of the analytical effort to establish the spectra of aerodynamic and geometric parameters on optimu turbines conducted under the previous task, a brief review of the literature was undertaken to acquire available experimental data on horizontal axis wind energy conversion systems. The purpose of this effort was to supplement the parametric study with experimental data on full scale and model wind turbines including performance, structural, and operational characteristics and other observations which could be useful in aiding in the selection of the test models. Moreover, some of these data were included with the wind tunnel test data on the limited number of models in this program for evaluating and correlating with the analytical wind turbine aerodynamic methodology.

Task 4 -Selection of Model Wind Turbine Configurations

A series of four basic wind turbine configurations were defined utilizing the results of the parametric study and the literature search. These $2.44 \mathrm{~m}$ ( $8 \mathrm{ft}$.) diameter models incorporated the geometric variables which have been shown to have the largest effects on performance and on structural design.

Task 5 - Structural Design of Wird Turbine Nodels

The structural design of the selected model blades was based on the use of the most appropriate materials, a retention configuration compatible with existing model propeller hubs, and a structure adequate for testing in the wind tunnel environment. In view of possible structural design constraints, some modification of the blade geometry established on aerodynamic considerations was required. Four different blade configurations were designed plus a counterweight for the one-biaded versions of two blade designs. Blade frequencies were calculated and design iteration undertaken to control placement of restricted operating zones due to critical frequencies which occurred within the test operating range. Each model was designed to minimize operating restrictions due to stall flutter. Detail drawings and templates suitable for manufacturing were prepared.

Task 6 -Predicted Performance of Selected Wind Turbine Configurations

The complete performance spectrum corresponding to the planned test points vas calculated for each model test configuration utilizing the appropriate analytical method to permit an exact comparison of predicted and measured performance. 
Task 7 - Manufacture of the Wind Turbine Models

In consideration of estimated costs and delivery dates, a qualified vendor was selected to manufacture the model wind turbine blades. Liaison was maintained with the vendor to assure adequate drawing definition and to. incorpcrate practical modifications which were indicated by the manufacturing process. Vendors were monitored to insure compliance with technical, schejule, and cost requirements.

A total of nine model turbine blades were manufactured to provide three wind turbine models of two blades each, one model each of three, two, and one blade with the same blade configuration, plus a counterweight. This combination provided siy configurations for the test program.

Task 8 - Wind Tunnel Test Program

The wind tunnel test program was conducted in the United Technologies Research Center's $5.49 \mathrm{~m}$ (18 ft.) throat subsonic wind tunnel. A special test rig and tower was provided for mounting the test turbines. The rig incorporated a turbine loading system mounted on the six-component main tunnel balance. The testing covered the complete operating envelope of each model turbine from no load to maximum power output or stall flutter limit with variations in blade angle and tip speed. The wind velocity was varied to show the effect of Reynolds number on performance.

This testing was accomplished on three models as discussed in Sction 10 below. In addition, the original plan called for further testing which was not completed when the frogram was terminated as the result of a blade separation accident in the wind tunnel. It is appropriate to mention these flanned tests siuce the corresponding analytical phase was accomplished and included in this report. The terminated portion of the program included, (1) testing the three blade, 30AF model with the plane of the turbine yawed over a series of angles to the wind velocity to establish the effect of wind direction on performance and on the resulting force and moment components, (2) investigating the effect of airfoil type on wind turbine performance using the two-blade, 60AF models with NACA 230XX airfoils and with NACA 44XX airfoils, respectively, (3) investigating the effect of surface roughness and waviness on the performance of the two-blade, 60AF model with NACA $230 \mathrm{XX}$ airfoils, and (4) defining the wake geometry of the two-blade, $30 A F$ model by flow visualization photograph of the wake under strobe-1ight. The test results from the first two items were to be compared to the corresponding predicted data presented in Section 9. The wake geometry was to have been incorporated into the prescribed wake program as discussed in Section 3.

Task 9 - Data Analyses and Evaluation

The experimental data are presented in the same wind turbine parameters and formats as the calculated data to permit a direct comparison for evaluating the Hamilton Standard and Skewed Wake Program adapted for application to wind turbines.

The test data on the three models tested are presented in Section 10 and corpared with the corresponding predicted data in Section 11 . In addition to the data acquired in this program, similar data from two other organizations have been included in the comparisions. 
Finally, the effect of number of hlades, hlade solidity and Revnolds number on performance as established by the testing is presented in wind turbire performance parameters.

\section{Task 10 - Identify Areas of Future Research}

As the final task, areas where additional research and development effort could provide further technological refinements have been identified.

The work undertaken to accomplish these tasks is discussed in the following text. 
SECTION 3

AERODYNAMIC DESIGN AND PERFORMANCE METHODOLOGY

\subsection{INTRODUCTION}

Derivation of wind turbine aerodynamics can be traced to the early theoretical work on propellers with recognition of the so-called "windmill" regime of operation. Many of the published theories deal with the case of energy extraction from the fluid medium as a logical extension of the well defined propeller case where shaft energy is absorbed to produce thrust. The accepted wind turbine aerodynamic design and performance prediction methods have been derived from some form of the propeller vortex theories. In these theories, the induced velocities are computed by summing the tangential and the axial velocity increments resulting from the bound and trailing vortex system developed on the blading as a function of input power." Then, with appropriate two dimensional airfoil data, the aerodynamic forces acting on a series of radial elements are calculated and integrated over the blade radius to establish the total forces.

It is the intent in this program to select appropriate, existing propeller and rotor theories for modification to wind turbine application. These programs include the Goldstein Propeller Method, the Prescribed Wake Program, the Rotor Wake Geometry Program and the Skewed Wake Program. The Goldstein method had previously been adapted for wind turbine aerodynamic design and performance prediction. However, additional refinement was accomplished under this program. This program has been utilized for the design of the model wind turbines and to compute the performance trends in the parametric study discussed below. This same program has been extended to cover variations in Reynolds number to predict the complete performance of the test models as tested in the wind tunnel. The Skewed Wake Program will be utilized to predict the forces and moments generated on a model wind turbine when operating with the axis of rotation yawed over a range of angles to the wind stream. The two vortex wake programs are relatively new developments and have not been utilized on a routine basis as has the Goldstein method. However, both represent an advancement in rotor aerodynamic modeling. Thus these methods have been included to assess their applicability to wind turbine design.

A brief description of each of these four aerodynamic programs and the modifications required to adapt each for wind turbine application is presented in the following text.

\subsection{GOLDSTEIN PROPELLER METHOD}

The Goldstein propeller design method is widely used throughout the propeller industry and is based on the work of Goldstein (ref. 1) and Locke (ref, 2). Goldstein obtained the exact sclution for the velocity field around an optimum, lightly loaded propeller with a finite number of blades. His model consisted of a rigid wake moving with a constant velocity. Locke applied the work of Goldstein to formulate a propeller vortex theory which includes the components of the induced velocity. Also, Locke suggested that the Goldstein method be applied without the limiting assumptions. This method when utilized with Hamilton Standard two dimensional airfoil data, shows good agreement with test data over the complete operating spectrum of propellers in forward flight. 
For application to wind turbine design, this same basic method has been modified to account for energy extraction rather than energy input to the fluid medium. Hütter (ref.' 3 ) has shown that the expanding wake of the wind turbine compared to the contracting wake of the propeller results in considerably less drop-off in circulation at the tips with a finite number of blades. This effect could result in blade loadings somewhat different from those based on Goldstein. However, from a comparison of the wind turbine performance data calculated by the Goldstein method and that in reference 3 , it is apparent that both results are similar and indicate that the wake expansion effect may be only secondary and can be neglected when using the Goldstein non-contracting wake method. However, further refinement of the method may be required when more experimental data have been obtained from this test program to compare with predicted performance. This empirical approach to method development and refinement has been applied throughout the history of propellers. Aerodynamic research at Hamilton Standard has effectively combined refinements of theory with experimental investigation to continually improve the aerodynamic design and performance prediction methodology.

The current method has been programmed on a high speed computer to permit extensive design analysis of each propulsor application in a brief time period. The applicability of the modified Hamilton Standard propeller strip analysis method to wind turbines has been substantiated by comparison with limited experimental data on wind turbines and ram air turbines. The calculated data generally agree with the experimental data in predicting the effects of variation in geometric and aerodynamic parameters on performance. An example of these comparisons is shown in figures $3-1$ and $3-2$. Performance is plotted in terms of power ratio versus velocity ratio for a range of blade angles on figure 3-1, with the corresponding rotor thrust (drag) presented as thrust ratio on figure 3-2. for a four bladed, 1.0 meter ( $3.25 \mathrm{ft}$. ) diameter wind turbine at wind velocities between $8 \mathrm{~m} / \mathrm{s}$ (18 MPH) and $20 \mathrm{~m} / \mathrm{s}(45 \mathrm{MPH})$. The experimental data were obtained from the work of Iwasaki presented in reference 4 .

Power ratio is defined as the ratio of output power to the wind power in a stream tube equal to the rotor diameter for a given wind velocity; i.e., $P R=$ Power Output $/\left(1 / 2 p\left(\pi D^{2} / 4\right) V^{3}\right)$. Velocity ratio is the ratio of turbine tip. speed to the wind velocity (VR $=T S / V)$. Similarly, thrust ratio is the ratio of the negative thrust or drag developed by the turbine divided by the wind dynamic force on the area swept by the turbine diameter; i.e:, $\mathrm{TR}=$ Thrust $/\left(1 / 2 \rho\left(\pi D^{2} / 4\right) V^{2}\right)$.

The comparisons presented in figures $3-1$ and 3-2 are for relatively low velocity ratios and large numbers of blades. These characteristics are generally not compatible with large wind energy conversion systems (WECS). As will be discussed later, these large systems operate at velocity ratios above 6 and incorporate only two or three blades for improved efficiency and cost effectiveness. The testing conducted under this program encompassed the maximum velocity ratio consistent with practical blade geometry (see Section 4). The resulting data will permit the modified Goldstein method to be evaluated for geometric and aerodynamic parameters associated with large WECS. 
This method has been utilized for the aerodynamic design and performance prediction of the wind turbines incorporated in the WECS study by General Electric and Hamilton Standard (ref. 5) and in a paper (ref. 6) on the performance of aerodynamically optimum wind turbines.

Under this contract effort, the computer program of the Goldstein method has been further refined for improved operation. $\Lambda$ iterative technique was revised to alleviate a functional problem and the two-dimensional airfoil test data packages for the NACA 230XX, the NACA 44XX and the new general aviation, GA(W)-2 airfoil series were extended to include Reynolds numbers ranging from those corresponding to full-scale, large wind turbines to the $2.44 \mathrm{~m}$ ( $8 \mathrm{ft}$. ) diameter models of this program. This extension was reauired for the parametric study (Section 4) and for predicting the performance of each model over the complete test range.

It should be pointed out that two operating conditions may be encountered which cannot be handled rigorously by the methodology developed to date. These are the vortex ring state of flow and vortex interference. The vortex ring state of flow is associated with an unstable, oscillatory type of flow caused by air interaction between freestream and wind turbine induced velocities. It occurs only when the freestream and induced velocities are of similar magnitude and opposed, as in the case of a helicopter in vertical descent. Second, the vortex interference occurs when the tip vortex of one blade passes close to the vortex of the following blade. It is expected that both of these conditions may occur near start-up. If possible, the wind tunnel program will investigate this region to establish the effect on start-up characteristics and to indicate the need for modification to the method.

\subsection{ROTOR VORTEX WAKE METHODS}

In order to improve method accuracy by eliminating the need for the fixed rigid wake geometry of the Goldstein theory, arbitrary wake geometries can be introduced through the use of finite filament vortex theory. Whereas the Goldstein theory, through the use of simplifying assumptions, is a closed form mathematical soiution, the finite filament vortex theory utilizes a numerical form of a solution.

The basic element in vortex theory is the vortex filament. Its effect on the induced velocity at an arbitrary point in space, the field point (A), is calculated by the classical Bict-Savart law, which relates induced velocity, $V_{A}$, to the filament circulation strength, $\Gamma$, and geometry as shown below.

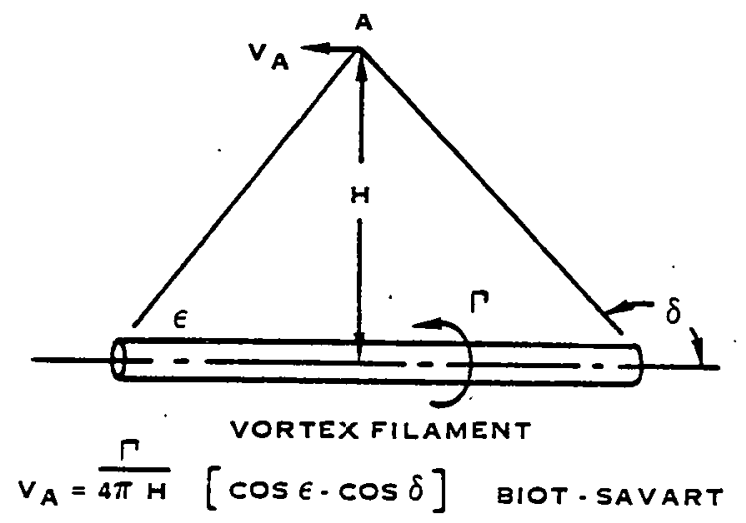


This methodology was first used successfully in aerodynamics many years ago in the development of wing horseshoe vortex theory and its associated induced inflow correction.

In the last five years vortex wake theory has been applied to helicopter rotors, references $7,8,9$, VTOL propellers, reference 10 , and now wind turbines.

The basic concepts can most easily be understood by applying vortex theory to the simplest case, a wing with constant circulation. If the wing is replaced by a lifting line on which the bound circulation and calculated. velocities are located, all of the induced downwash velocity is caused by the tip trailing vortices.

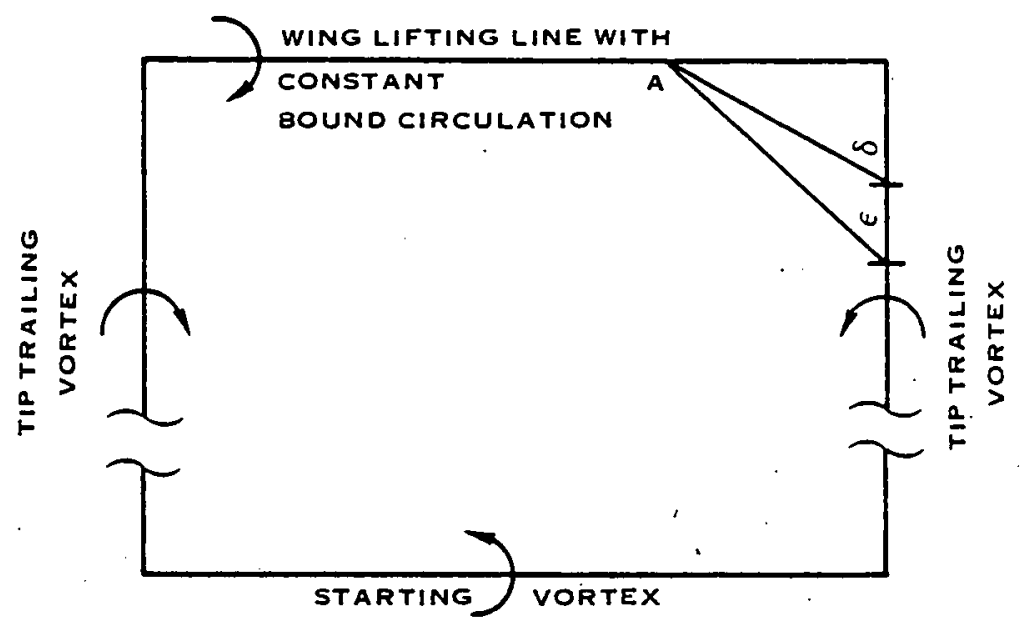

Referring to the above figure, it is noted that the bound circulation does not contribute to the induced velocity since the lifting line passes through its center. The starting vortex moves far enough downstream to where its effect is zero. For a numerical solution, the trailing vortex is then broken up into $\mathrm{N}$ filament segments of equal circulation, $\Gamma$, one such filament being shown in the figure above. The Biot-Savart equation is then applied to each filament segment and summed for each field point (blade station).

One added complication occurs since the calculated induced flow changes the velocity vector diagram and the wing angle of attack. When using two dimensional airfoil data there is an iteration between circulation and induced velocity that must be satisfied using both the Biot-Savart equation and the Kutta-Joukowski equation below:

$$
\Gamma=\frac{\text { CHORD }}{2} C_{\mathrm{L}} \cdot \mathrm{W} . \quad \begin{aligned}
\mathrm{C}_{\mathrm{L}} & =\mathrm{f} \text { (Angle of Attack) } \\
\mathrm{W} & =\mathrm{f}\left(\mathrm{V}_{\mathrm{A}}, \mathrm{V}_{\mathrm{O}}\right)
\end{aligned}
$$

When vorter: theory is applied to rotors, propellers or wind turbines, there are, as expected, many added complexities, especially where the induced velocities are large with respect to freestream velocity. Such is the case for hovering rotors, static or low speed propellers and wind turbines. The major problem occurs in determining the wake shape or the proper location of the trailing vortex segments. For example, the tip vortex is the major contributor to 
inflow since its circulation is proportional to $\mathrm{d} \Gamma / \mathrm{dr}$ which is usually maximum at the rotor tip. However, its precise location in space is difficult to establish by analysis. For a static propeller the tip vortex does not move straight back but in a contracted helical path of varying pitch; conversely the tip vortex path of a wind turbine expands outward.

Thus for helicopter rotors and aircraft propellers, two approaches to defining the wake shape have been developed. The first utilizes flow visualization of the wake from which generalizcd wake parameters have been derived for incorporation in the basic vortex method. In the second approach, the wake shape is analytically defined. Both definitions have been adapted for the expanding wake of a wind turbine.

As pointed out previously, three methods based on the vortex filament wake definition are being investigated in this program. The Skewed Wake Program for inclined flow, and the Prescribed Wake Program utilizing the empirically defined wake shape and the analytically defined rotor wake shape for normal operation are discussed below.

\subsection{SKEWED WAKF PROGRAM}

This method calculates the performance of a rotor when the freestream velocity is at an angle to the rotor shaft centerline. The method was originally developed as a helicopter rotor performance method by United Technologies Research Center (UTRC) and later converted to a VTOL propeller method by Hamilton Standard.

The wake shape utilized in the program is a constant diameter helix skewed at an angle to the rotor centerline. The pitch of the wake is independent of blade radius and advances at a velocity equal to the vector sum of the freestream and average momentum velocities. Once the wake is formed and located, by use of the Biot-Savart equation, the axial induced velocities are computed as a function of the azimuthal and radial location of the blade. A momentum balance is achieved between the wake position and the thrust by iterative adjustments of wake position. Once the iteration is successful and the induced velocities calculated, six component rotor forces and moments are then calculated.

\subsection{PRESCRIBED WAKE PROGRAM}

The Prescribed Wake Program requires as input the actual wake shape of the rotor which can be obtained in one of two ways.

1. By an analytical method (see 3.6 below).

2. From flow visualization test data.

Approach 2 was done successfully in reference 10 by using smoke rakes and measuring wake shapes from photographs. A successful static propeller performance prediction method evolved from the generalization of smoke picture wake geometry inputted into the Prescribed Wake Program. This flow visualization technique will be attempted for wind turbines during the test program.

The Prescribed Wake Program developed by UTRC as a hovering helicopter rotor method, has been extended by Hamilton Standard to propellers and wind turbines including the effect of wind velocity. The program is described in detail in reference 10. 
Briefly, the program incorporates a wake consisting of a finite number of trailing vortex filaments of inputted position. The Kutta-Joukowski and Biot-Savart relations are solved in a matrix solution which yields the blade circulation and corresponding induced velocities. The blade velocity diagram is then constructed and with the use of two-dimensional airfoil data, the loading distribution is obtained. The total thrust and horsepower are ti:sn established through integration.

\subsection{ROTOR WAKE GEOMETRY PROGRAM}

This method, developed by UTRC will calculate the wake shape of a rotor by iterative techniques. Initial inputs to the program are circulation distribution and an assumed wake shape.

By the use of the Biot-Savart law induced, velocities are calculated in the wake. These velocities are integrated over a small increment in time to define a new wake shape. A converged wake shape is obtained when it remains unchanged. The wake shape is then inputted into the Prescribed Wake Program (described above) yielding a new circulation distribution. The above steps are repeated until a compatible wake geometry and circulation distribution is obtained. The method has been recently adapted to calculate wind turbine wake shapes. The first converged wake shape has been calculated and awaits the first calculation of the circulation using the Prescribed Wake Program. The initial wake geometry calculation is described in detail below.

The conversion and checkout of the UTRC rotor wake geometry analysis for application to wind turbines was completed. A modification to the computer program for the handling of program mass storage completed the conversion to the UNIVAC 1110 computer system. A check case, based on a Hamilton Standard wind turbine design and a blade wake circulation distribution from the prescribed wake propeller program has been run to compute the tip vortex geometry. Although experimental data are not available for correlation, the results appear reasonable considering that the cylindrical coordinates of the tip vortices from both blades converged to an identical time-independent solution and the wake is predicted to smoothly expand. For the check case a wake expansion of nine percent of the wind turbine diameter is predicted at a distance of one radial length. from the plane of rotation (two wake revolutions). At a distance of two radial lengths the predicted wake expansion is twelve percent, which is approximately its limiting value.

\section{DESCRIPTION OF CHECK CASE AND RESULTS}

The wind turbine investigated in the check case had the basic design and operating parameters listed below:

$\begin{array}{lll}\text { Number of blades } & 2 & \text { Wind Speed } 6.7 \mathrm{~m} / \mathrm{s} \cdot(13 \mathrm{kt}) \\ \text { Diameter } & 30.5 \mathrm{~m}(100 \mathrm{ft}) & \text { Tip Speed } 67.1 \mathrm{~m} / \mathrm{s}(219.8 \mathrm{fps})(42 \mathrm{rpm}) \\ \text { Airfoil } & \text { HSD Series } 16 & \text { Velocity Ratio }=10,\end{array}$

Planform . Early HSD Design 
The UTRC rotor wake geometry analysis as adapted for the wind turbine was applied to compute the tip vortex geometry. The basic approach of this analysis involves the following procedure. First, an undistorted wake model is defined along with the distribution of circulation strengths of the various vortex elements used to represent the wake. The circulation strengths are obtained from the blade circulation distribution previously calculated using the HSD/UTRC Prescribed Wake Program which has also been adapted for the wind turbine. The Biot-Savart law is then applied to determine the velocities induced by each vortex element at the end points of all vortex elements. These distorting velocities are then numerically integrated over a small time increment to obtain new wake element positions. The process of alternately computing new velocities and positions is continued as the blades rotate to equally spaced azimuth positions. The final time step is reached when a converged distorted wake geometry is attained.

Front and top views of the tip vortex geometry calculated in the check case are presented in figure 3-3. For clarity, the tip vortex of only one blade is shown (the vortex of the second blade is identical except for the 180 degree phase shift). The straight vortex elements used to represent the rolled up tip vortex filament are shown rather than the actual curved elements which can be easily perceived by mentally fairing through the element end points. The undistorted tip vortex geometry used to define the initial tip vortex coordinates in the wake geometry analysis are included in figure 3-3 for comparison. The predicted tip vortex geometry defines a wake boundary which is shown to smoothly expand from the cylindrical boundary of the undistorted wake. A predicted expansion of the wake is consistent with what is expected for the unpowered wind turbine.

The tip vortex coordinates are cross plotted in figure 3-4. The wake boundary is depicted by the radial versus axial coordinate plot. For the wind turbine design and velocity ratio of the check case, a wake expansion of nine percent is predicted at a distance of one radial length from the plane of rotation. At a distance of two radial lengths, the wake expansion is twelve percent. It appears that the wake expansion is asymptotically approaching a limiting value of approximately thirteen percent. The axial coordinate is shown to linearly vary with tip vortex azimuth. This differs from the characteristic tip vortex feature of 'a powered propeller or rotor which is the increase in the slope of the axial coordinate - azimuth curve. following the passage of the following blade. Since the tip vortex of the wind turbine does not pass under the following blade, an axial acceleration produced by that blade is not predicted.

It is noted that an iteration between the Prescribed Wake Program and the wake geometry analysis remains to be conducted to compute the wind turbine power generated based on the predicted wake geometry and insure the consistency of the performance and wake solutions. However, based on the predicted wake geometry results, the influence of tip vortex distortions relative to the undistorted helical geometry on performance (generated power) is anticipated to be small due to the following reasons:

1. The wind positions the tip vortices sufficiently behind the wind turbine to make the distortion of each point on a tip vortex small relative to its distance to the wind turbine blades. 
2. The distortions are in a direction away from the wind turbine. This is opposite to the tip vortex distortions for a powered propeller or rotor where the tip vortex is distorted toward the blade which can lead to large aerodynamic interference effects where the tip vortex of one blade passes close to the following blade.

3. Since the wake expands rather than contracts as for a powered propeller or rotor, the tip vortices do not pass directly under the blades. This eliminates the strong velocity gradient near the blade tip associated with opposite directions of the axial component of induced velocity on opposite sides of a near tip vortex. This wake expansion should result in a smoother blade axial velocity distribution and a lesser influence on power. 


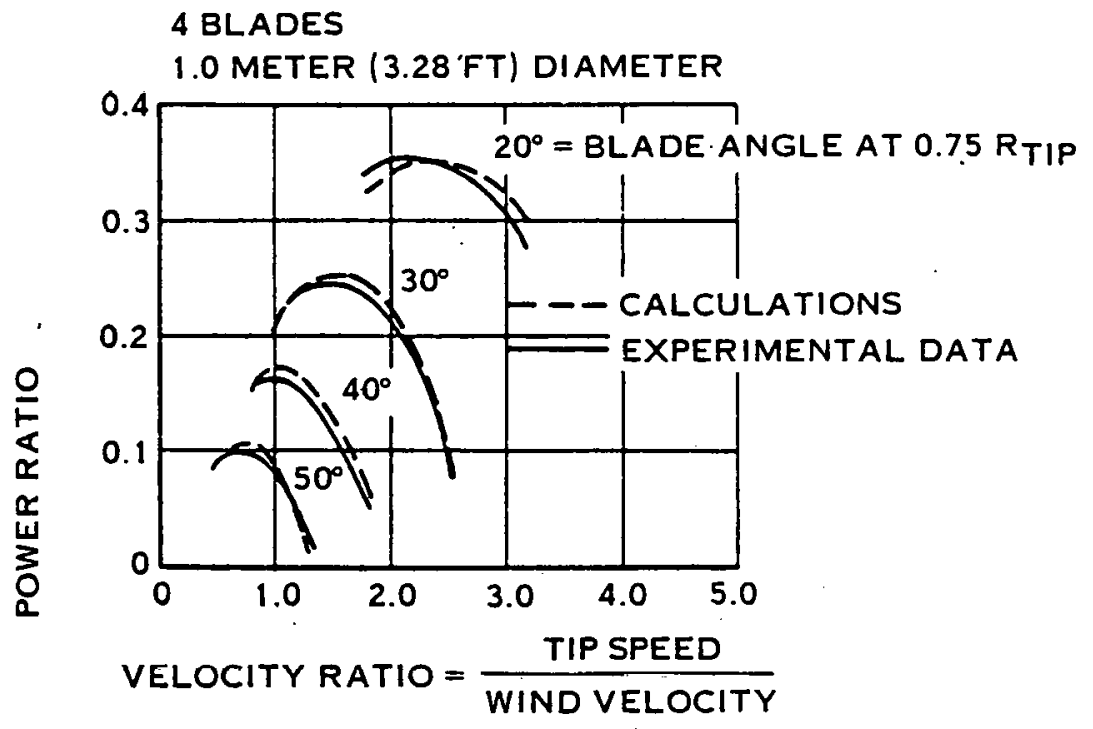

FIGURE 3-1. COMPARISON OF POWER RATIO CALCULATIONS AND EXPERIMENTAL DATA

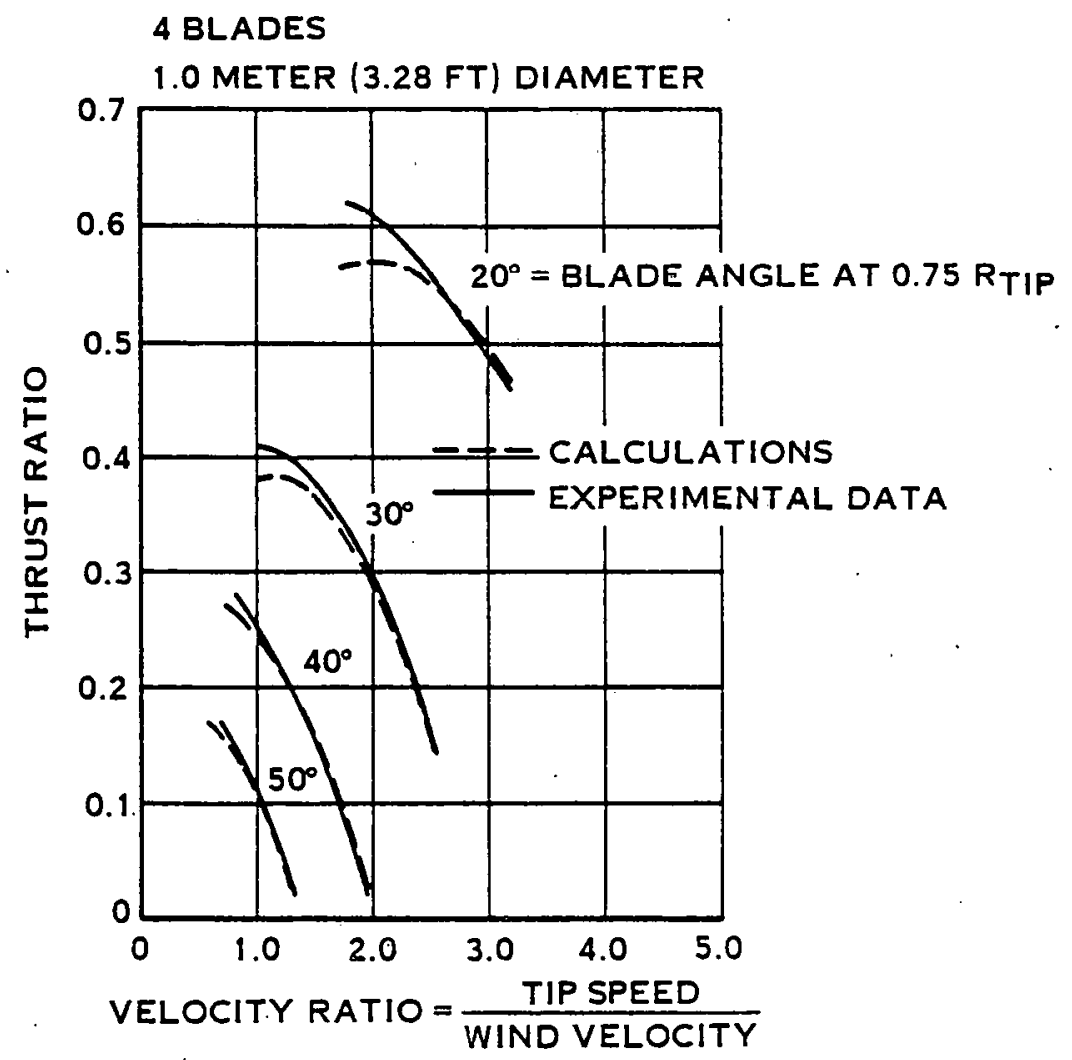

FIGURE 3-2. COMPARISON OF THRUST RATIO CALCULATIONS AND EXPERIMENTAL DATA 
- - - INITIAL UNDISTORTED TIP VORTEX

PREDICTED, UTRC ROTOR WAKE GEOMETRY ANALYSIS

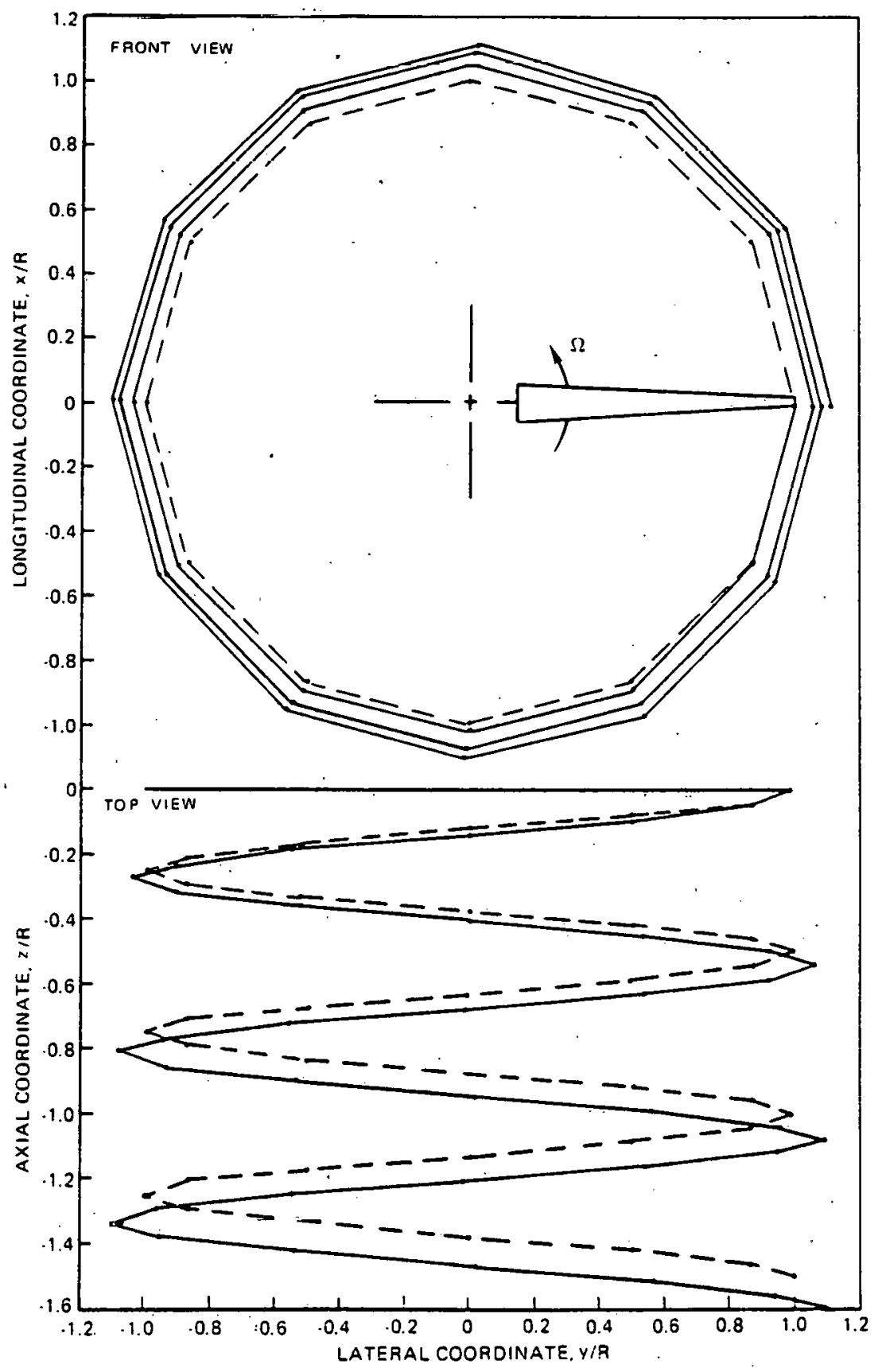

FIGURE 3-3. TIP VORTEX GEOMETRY FOR WIND TURBINE BLADE 


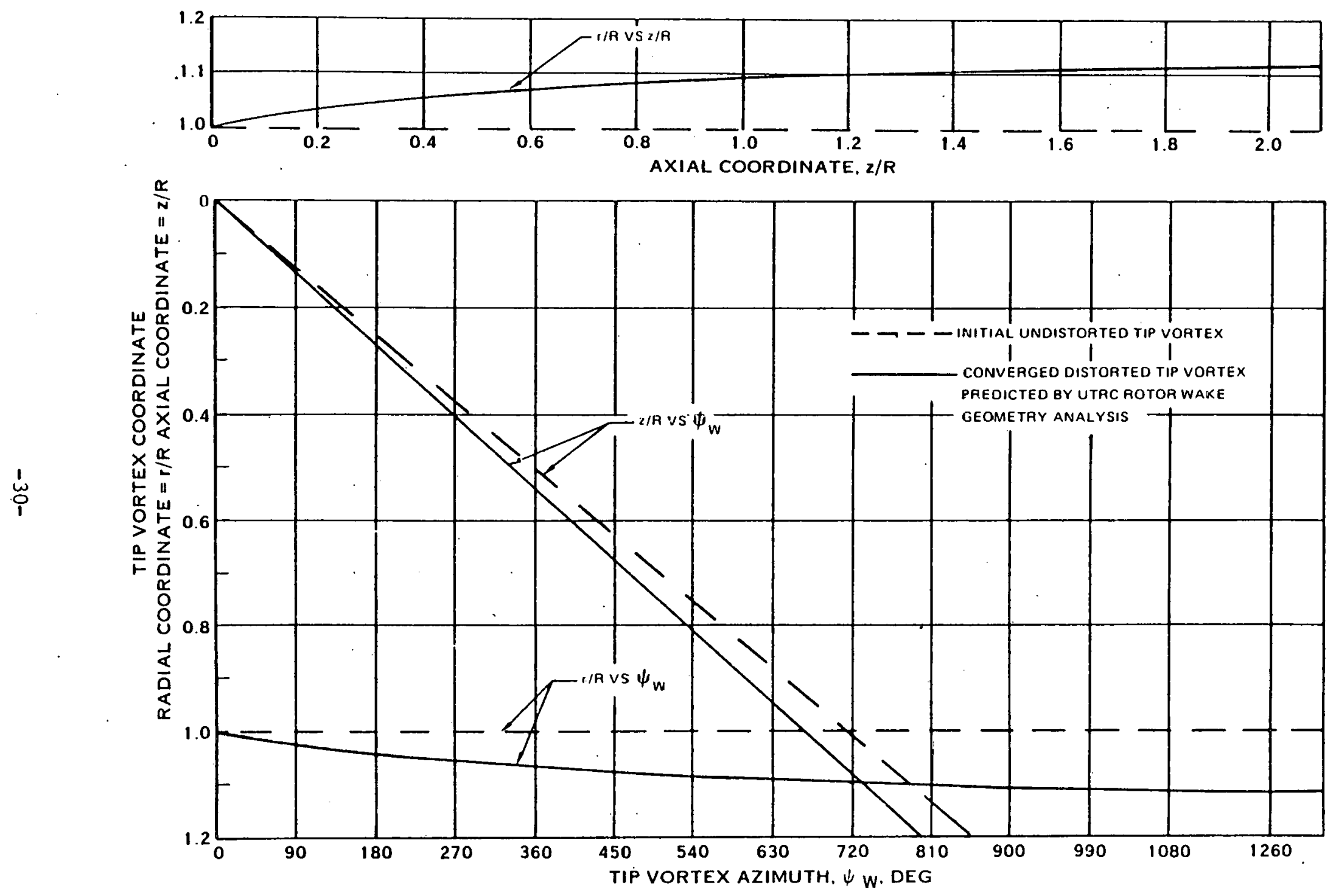

FIGURE 3-4. TIP VORTEX COORDINATES FOR WIND TURBINE 
SECTION 4

WIND TURBINE PARAMETRIC PERFORMANCE STUDY

\subsection{INTRODUCTION}

The parametric performance study was undertaken to investigate the effects of the prime wind turbine aerodynamic and geometric variables on performance covering the operating spectrum permitted by practical blade geometry and consistant with cost effective wECS concepts. The results of this analytical study would then serve to provide a basis for selecting the model wind turbines incorporating those variables deemed most significant and in need of experimental investigation.

Prior to discussing the results of this study it is appropriate to first review the aerodynamics of wind turbines with particular focus on the interrelationships of the aerodynamic and turbine geometric variables as influenced by operating requirements of the complete system.

\subsection{AERODYNAMICS OF WIND TURBINES}

The performance of any turbine can be completely defined in terms of the two basic parameters defined in the previous Section, i.e., power ratio and velocity ratio. In terms of these parameters, Glauert (ref. 11) has defined the variation of ideal envelope performance of wind turbines with velocity ratio and has shown that power ratio approaches a maximum value of 0.592 at high values of velocity ratio as shown in figure 4-1.

The velocity ratio combines the two most important variables affecting the rotor design, the diameter and the wind velocity, as well as the most important drive train variable, the shaft rpm. This latter variable is particularly important when the wind power rotors are applied to the generation of a.c. electrical systems. Thus the primary aerodynamic and geometric parameters as well as power output can be plotted versus velocity ratio. The blade geometry parameters having the greatest effect on performance and turbine costs are number of blades and airfoil section as expressed by design lift to drag ratio, L/D. The effect of these parameters on peak rotor performance has been defined (ref. 5 and 6 ) for a matrix of optimum rotors and a range of velocity ratios using the Goldstein propeller method. The resulting performance envelope for dragless wind turbines, one to four blades are shown in figure 4-1. This figure indicates that the performance of these wind turbines increases with velocity ratio and with number of blades. It should be noted that the theoretical performance of the one bladed wind turbine has not been well defined. At high velocity ratios the performance of the two to four bladed wind turbines approaches to within two to four percent of the Glauert ideal. Figure 4-2 presents the effect of airfoil operating lift to drag ratio (L/D) on the performance of optimum two bladed turbines for the velocity ratio range investigated. The curve shows a very strong adverse effect of decreasing L/D on.performance which becomes more severe with increasing velocity ratio. Thus the need to design for L/D levels above 80 is obvious from a study of this figure. Similar trends with $L / D$ can be shown for other blade numbers. 
The corresponding trend in blade width distribution with velocity ratio is presented for the matrix of optimum wind turbines in terms of a solidity parameter, Activity Factor (AF), for a range of operating lift coefficients and number of blades in figure 4-3. AF is a blade area weighted solidity term which accounts for the radial distribution of chord width. Thus:

$$
A F=\frac{100,000}{16} \int_{\text {hub }}^{\text {tip }} b / D(r / R)^{3} d(r / R)
$$

where $b$ is the section chord, $D$ is the wind turbine diameter, $r$ is the local radius and $R$ is the radius of the wind turbine. In general, increasing $A F$ means wider blades as depicted by the blade sketches in figure 4-7. Thus, this parameter significantly affects structural design and cost as well as performance.

The design point performance and blade geometry for optimum turbines may be selected from these basic charts for any design wind velocity and wind turbine tip speed as functions of number of blades and design L/D. From a study of figures $4-3$, it is observed that as the design velocity ratio increases for a given wind turbine diameter, solidity decreases to remain optimum. Since the corresponding blade structural design and construction techniques may become more difficult as blade chord becomes extremely narrow, a trade-off between performance and costs is implied as the options of reduced number of blades or non-optimum configurations are considered. Preliminary structural design analysis has indicated a minimum $A F$ of 15 as shown in figure 4-3. Moreover, the step-up gearing required for the generation of a.c. electrical power becomes less of a design problem and cost item with increased design velocity ratio which further favors higher tip speed, lower solidity turbines. It should be pointed out that the transmission requirement may not be a big factor in other wind power generator applications.

With the low AF structural limit of 15 , the trends in figure $4-3$ show that to design for velocity ratios above 12 in order to minimize gearing size, weight and costs, operating lift coefficients $\left(C_{L}\right)$ less than 1.5 are required. Moreover, the number of blades needs to be the minimum consistent with acceptable performance to avoid impractically low activity factor blades. From these considerations, the selection of practical optimum turbines can now be simplified by defining performance and blade geometry boundaries on the operating envelopes of figures $4-1$ and $4-2$.

At both ends of the power ratio/velocity ratio spectrum, limitations can be defined which place bounds on the useful range of design velocity ratio. The low velocity ratio is bounded by rapidly reduced performance and high rotor solidity, while the high velocity ratio is bounded by structural limitations on low $\mathrm{AF}$ and reduced performance.

As mentioned previously, the curves in figure 4-3 indicate a trade-off in number of blades, operating $C_{L}$ and design velocity ratio in view of a low limit on AF. This trade-off can be somewhat resolved by consideration of the data in figure 4-4 (ref. 12, 13, 14 and 15). Here the airfoil section lift to drag ratio trend with operating lift coefficient is presented for a variety of airfoil types. The data is for 3 million Reynolds number which is representative for 22.9-45.7 meter $(75-150 \mathrm{ft}$ ) diameter wind turbines and mean wind velocities $5.4-6.7 \mathrm{~m} / \mathrm{s}$ (12-15 MPH) range. 
The selection of airfoil type is strongly influenced by operating lift coefficient and design Reynolds number. Although performance improves with high values of $L / D$, the corresponding high values of operating $C_{L}$ rapidly reduce solidity below the limits established in figure $4-3$. Thus the selection of the airfoil type must be based on the maximum L/D consistent with practical blade geometries and performance levels. As previously pointed out, cost considerations of the step-up gear lead to high values of velocity ratio which require low AF blades. Thus from figure 4-3 an operating lift coefficient of 1.0 appears to be a good choice for two bladed wind turbines. For higher blade numbers the operating $C_{L}$ would need to be reduced. A one blade wind turbine appears to be required for an operating $C_{L}$ of 1.50 . As indicated in figure 4-4, conventional airfoils like the NACA $230 \mathrm{XX}$ series, the NACA $44 \mathrm{XX}$ and the NACA 6 Series show $\mathrm{L} / \mathrm{D}$ in the 100-120 range at an operating $C_{L}$ of 1.0. Advanced airfoils like the Wortmann and Liebeck designs show L/D's above 150 at an operating $C_{L}$ of 1.50 , whereas sail type airfoil data indicate unacceptably low L/D's (below 20).

On the basis of the data shown in figure 4-4, limitations on the maximum value of velocity ratio for each number of blades due to the activity factor limit of 15 . is shown in figure $4-5$ for a range of operating $C_{L}$ 's. From an inspection of this figure, it should be noted that the highest values of velocity ratio are obtained with low operating $C_{L}$ 's with lower values of this parameter as number of blades are increased. Since the maximum L/D values are associated with the higher values of operating $C_{L}$, it is apparent that there is a trade-off between design velocity ratio and $\mathrm{L} / \mathrm{D}$.

Accordingly, the minimum 15 AF constraint on velocity ratio for the operating CI range from 0.5 to 1.5 as shown in figure 4-6 along with a lower velocity ratio limit of 6 for two blade wind turbines. The high velocity ratio boundary indicates the performance level associated with the $L / D$ level at each operating $C_{L}$. Thus the highest value of velocity ratio corresponds to a low L/D with low performance while the highest performance corresponds to a lower value of velocity ratio. At this point, the optimum trade-of $f$ appears to be an operating envelope for practical turbines between a low velocity ratio of 6 and the 15 AF boundary at high velocity ratios.

The variables can be further reduced by the selection of an airfoil type. Although the high L/D Wortmann section warrants further study and research, the limited experience with these airfoils and their reported sensitivity to surface conditions as well as the limitation on velocity ratio at the required high operating $C_{L}$ would lead to the conclusion that these airfoils should not be utilized for first generation wind rotors: On the other hand, well proven NACA airfoils including the $230 \mathrm{XX}$ and $44 \mathrm{XX}$ series show L/D's at the 120 level, resulting in high wind turbine power ratios and design velocity ratios as high as 16. Moreover, these airfoils (ref. 12) have demonstrated high L/D over a. wide range of lift coefficients at Reynolds numbers appropriate to large wind turbines. Thus either of these NASA airfoil series may be selected for high performance and minimum risk. It should be pointed out that the $\mathrm{L} / \mathrm{D}$ levels for all airfoils are significantly reduced by surface roughness.

Thus it is seen from a review of figure $4-6$ and the foregoing discussion on airfoils that the spectrum for peak performance for horizontal axis, propellertype wind turbines lay in a rather narrow band bounded by velocity ratios of 6 and 18 and by airfoil lift to drag ratios of 80 and 160 . Up to this point, the discussion has dealt only with the design point for a matrix of optimum 
wind turbines. Of course, under actual operation the wind turbine will be operating at peak efficiency only occasionally, particularly for systems generating electricity with synchronous generators, due, to the variation in wind velocity. Thus the off-design performance, particularly in wind velocities below the rated value, has a strong influence on the yearly output power of the system. Accordingly, in the parametric performance study discussed below, the complete performance characteristics of the wind turbine as influenced by aerodynamic and geometric variables has been investigated. Moreover, in establishing the most cost effective blade configuration, it is necessary to consider that the optimum shape for structurally adequate designs and minimum cost fabrication techniques may differ from the aerodynamic optimum shapes. Thus non-optimum wind turbines were also investigated.

\subsection{WIND TURBINE PARAMETRIC PERFORMANCE STUDY}

In the foregoing general discussion of wind turbine aerodynamics, the relationships of the aerodynamic and the geometric variables were brought into proper perspective. With this picture in mind, it is now appropriate to discuss the results of the parametric performance study. As indicated above, the analysis based on the Hamilton Standard wind turbine performance method (Goldstein) covered the full operating range from start-up to blade stall for each geometric parameter investigated. Both optimum and nonoptimum aerodynamic configurations were considered. The results are presented in terms of aerodynamic parameters, i.e., power ratio versus velocity ratio and, for a typical annual wind regime in terms of the annual energy output. This latter presentation is perhaps the most meaningful measure of the effect of turbine geometry since it is the real end product of the system.

Following considerable preliminary review of previous aerodynamic studies, available literature and structural requirements, the following parameters were investigated.

\author{
- Blade Activity Factor \\ - Number of Blades \\ - Blade Planform Distribution \\ - Blade Thickness Distribution \\ - Blade Twist Distribution
}

\author{
- Blade Airfoil Section \\ - Reynolds Number \\ - Roughness \\ -Waviness \\ - Blade Root Cut-off
}

Obviously, several of these same variables were investigated under the contract of reference 5. Accordingly, where appropriate, the results of this reference study were used to supplement the parametric study being. discussed herein. However, the focus of the current study is on a family of near-optimum wind turbines, whereas the reference study covered somewhat random configurations which varied from the optimum as a broad spectrum of structural and fabrication concepts were investigated. It should be pointed out that on the basis of the reference 5 program findings, the filament winding fabrication process will require no significant compromise to optimum blade geometry. Thus basing this program on near-optimum geometry is entirely consistent with current concepts for cost effective designs. 
A discussion of variations in each of the prime parameters listed above on wind turbine performance and operation is presented below.

\subsubsection{Blade Activity Factor}

On the basis of the operating envelope and solidities established above; a series of near-optimum two bladed wind turbines of 15 to $60 \mathrm{AF}$ were designed to span a range of velocity ratios. The $15 \mathrm{AF}$ turbine was selected as the low AF limit because of structural limitations as previously discussed and the $60 \mathrm{AF}$ was selected as the high AF limit because it corresponds to the minimum velocity ratio on figure 4-6. These blades were designed utilizing the Hanilton Standard propeller method modified to account for energy extraction rather than energy input to the fluid medium as discussed in Section 3. The distributions of planform (fig. 4-7), thickness (fig. 4-8), and twist shape (fig. 4-9) are optimum with deviations from the optimum in the shank region to represent more realistic distributions for structural. reasons. The planforms with the associated $A F$ and design velocity ratio for peak power ratio are shown in figure 4-7. AF is reduced with increased design velocity ratio as predicted in figure 4-3. As AF is increased, the amount of twist required is also increased as is shown in figure 4-9. The well proven NASA Series $230 \mathrm{XX}$ airfoil family was selected because these airfoil sections have demonstrated high $\mathrm{L} / \mathrm{D}$ over a wide range of lift coefficients at Reynolds numbers appropriate to large wind turbines. The airfoil selection is further discussed under the section on airfoils.

For these near-optimum wind turbines, the power ratios for the design blade angle were plotted versus velocity ratio in figure 4-10. As previously stated the peak power ratio is essentially constant over the entire AF range.

The data in figure 4-10 were tabulated for use in a computer program to investigate the annual wind turbine energy output for a representative yearly wind spectrum (fig. 4-11) and a range of mean velocities from $3.35 \mathrm{~m} / \mathrm{s}(7.5 \mathrm{MPH})$ to $11.8 \mathrm{~m} / \mathrm{s}(25 \mathrm{MPH})$. The data of figure $4-11$ was compiled by NASA Lewis. Research Center. This curve shows that for a given mean value, the wind velocity attains high values only a relatively few hours out of a year compared to the mean velocity. This presentation was included to show the effect of design velocity ratio and $\mathrm{rpm}$ on the annual energy output of the family of wind turbines.

Based on the preliminary design analysis presented in reference 5 , a 57.9 meter $(190 \mathrm{ft})$ diameter turbine with a 15 meter $(50 \mathrm{ft})$ ground tip clearance was selected as a reference size for this investigation into the effect of $A F$ on performance. By interpolation, an $8.0 \mathrm{~m} / \mathrm{s}(18 \mathrm{MPH})$ mean wind duration curve (fig. 4-11) was uecd with a rated veloc1zy of 11$) .3 \mathrm{~m} / \mathrm{s}$ ( $23 \mathrm{MPH}$ ). These velocities are based on measured values at 9.1 meters $(30 \mathrm{ft}$ ) above the ground and were reflected to a velocity at the turbine shaft by the following equation:

$$
\left.v_{(\text {shaft })}=v\left(\frac{\left(\frac{D}{2}+K_{1}\right.}{K_{2}}\right)\right)^{167}
$$




$$
\text { where } \begin{aligned}
\mathrm{K}_{1} & =15 \mathrm{~m}(50 \mathrm{ft}) \\
\mathrm{K}_{2} & =9 \mathrm{~m}(30 \mathrm{ft}) \\
\mathrm{V}(\text { shaft }) & =\mathrm{m} / \mathrm{s}(\mathrm{MPH}) \\
\mathrm{V} & =\mathrm{m} / \mathrm{s}(\mathrm{MPH}) \text { at } 9 \mathrm{~m}(30 \mathrm{ft})
\end{aligned}
$$

The velocity at the turbine shaft was used for the study to represent a mean value of the wind shear over the wind turbine diameter since it was shown in reference 5 that the wind shear has a small effect on performance.

The variations of power output for a one year time period were computed. An example is presented on figure 4-12. The assumption was made that these were zero friction systems. Moreover, once rated velocity is reached, the power output remains constant by spilling power with variable pitch blading or by tower turning.

The annual energy output is obtained by integrating the curve (fig. 4-12) of power versus hours. The kilowatts corresponding to the rated velocity and the corresponding kilowatt hours/year are plotted for a range of rpm's on figure 4-13.

It may be noted, that the maximum annual output and rated kilowatts are independent of $\mathrm{AF}$, $1 . e$. , the $15 \mathrm{AF}$ through $60 \mathrm{AF}$ wind turbines produce almost identical yearly output and rated kilowatts although different rpm's are required. This is clearly shown in figure 4-13. Thus it appears that, the selection of a rotor AF for a given WECS installation should be made for minimum cost per unit of power rather than overall performance. This selection process must consider gearing and transmission effectiveness as well as turbine performance.

The $30 \mathrm{AF}$ wind turbine was used to investigate the effects of the other parameter variations. Thus, it is referred to as the "basic turbine".

\subsubsection{Number of Blades}

The study discussed above for AF variations was repeated for the basic $30 \mathrm{AF}$ turbine for a range of number of blades from 1 to 4 . The effect of varying the number of blades of the "basic turbine" is shown as a function of power coefficient and velocity ratio in tigure 4-14. The calculatlon for the vue bladed turbine is shown as a dashed line because of the uncertainty in the calculation procedure. As was previously predicted, the peak power ratio increased with increase in number of blades. The rated kilowatts and annual output powers are plotted in figure 4-15. As for the peak power ratio, the rated kilowatts and annual output are a direct function of number of blades, decreasing as number of blades is reduced. Again the curve for the one bladed turbine is dashed because of the uncertainty of the calculation. It is apparent from figure 4-15 that the performance gains of 3 and 4 bladed wind turbines over the 2-blades are only 3 and $4 \%$, respectively. This performance increase can be reflected as a diameter reduction of 1 and $2 \%$, respectively, for the same performance as the 2-bladed wind turbine. The performance of the one bladed turbine is approximated as $30 \%$ lower. Since the calculated performance may be inaccurate due to the uncertainty of the analytical model, the one bladed wind turbine will be included in the test program. By increasing the diameter, the annual energy output of the one-bladed wind turbine can be increased and may still be economically competitive with a turbine of more blades since the blades are the major cost element of the wind energy conversion system. 


\subsubsection{Planform Distribution}

The effect of blade chord distribution on performance has been evaluated for rectangular blades and semi-trapezoidal blades as defined on figure 4-16. The semi-trapezoidal blades are similar to the basic blades over the outer $50 \%$ of the radius. It can be seen from figure 4-17 that there is a slight reduction in peak power ratio for the semi-trapezoidal and a large reduction plus a shift to a higher velocity ratio for the rectangular planform. This can be reflected as a $1 \%$ reduction in annual energy output due to the semi-trapezoidal planform and a $13 \%$ reduction in annual energy output due to a rectangular planform as shown in figure 4-18. Thus it can be concluded that devlacions from the optimum planform over the inner $50 \%$ of the blade span cause only small reductions in performance.

The study of reference 5 indicates that the performance penalty in deviating from the optimum planform to the rectangular planform is much reduced as the $\mathrm{AF}$ is increased from 30 to 60 . Thus, it can be concluded that the higher the design velocity ratio and consequently reduced $A F$, the greater is the performance penalty for deviating from the optimum trapezoidal planform.

\subsubsection{Blade Thickness Distribution}

The effect on power ratio of successively increasing the thickness ratio distribution in the manner shown in figure 4-19 is presented in figure 4-20 and on the annual energy output is shown in figure 4-21. It can be seen that the annual energy output is reduced $3 \%$ by increasing the $\mathrm{h} / \mathrm{b}$ to .18 and $10 \%$ by increasing the $\mathrm{h} / \mathrm{b}$ to .24 . For structural considerations and or cost reduction, the thickness ratio can be varied slightly in the tip region and to a greater extent in the mid-portion as shown in figure 4-19. It can be seen from figures 4-20 and 4-21 that the annual power output is reduced $2 \%$. Thus, the general trend is to reduce performance with increasing thickness. However, thickness is a powerful factor in reducing the weight and cost of hollow blade structures.

\subsubsection{Blade Twist Distributions}

The twist distributions shown in figure 4-22 were studied. Variations 1 and 2 incorporate changes in shank twist only. Version 3 eliminates the twist in the tip region and version 4 has no twist. It can be seen from figure 4-23 that the twist changes do affect the power ratio versus velocity ratio with the no-twist version being the poorest performer. It is shown in figure 4-24 that the large twist change in the inner $50 \%$ of the blades reduces annual energy output $2 \%$. Eliminating the tip twist also reduces annual energy output $2 \%$. However, there is a $7 \%$ loss in annual energy output with no twist. Thus, twist has a significant effect on performance.

As for the planform variation, in the study of reference 5 , it was found that at $60 \mathrm{AF}$, the reduction in annual output due to no twist was significantly less than that shown at $30 \mathrm{AF}$. Thus, as AF is reduced, the need to incorporate twist becomes essential for high performance.

\subsubsection{Airfoil Sections}

As was shown in figure 4-2, wind turbine performance is improved as lift to drag rat1o, $L / D$, is increased. The magnitude of $L / D$ is a function of airfoil section and operating lift, $C_{I}$, as shown on figure 4-4. As indicaced in this figure, increases in $L / D$ are obtained by increasing $C_{L}$. . Conventional airfoils 
like the NASA 230XX Series (ref. 12), the NACA 44XX (ref. 12) and the NACA 6 (ref. 12) Series show $L / D^{\prime}$ 's in the 100-120 range at an operating $C_{L}$ of 1.0. Advanced airfoils like the Wortmann (ref. 13) and Liebeck (ref. 14) designs show L/D's above 150 at an operating $C_{L}$ of 150 , and the Whitcomb GA(W) Series (ref. 15) airfoils show 85 to $90 \mathrm{~L} / \mathrm{D}^{\prime} \mathrm{s}$ for .8 to $1.0 \mathrm{CL}$, whereas sail type airfoil data indicate unacceptably low L/D's.

As previously stated, the selection of airfoil type is strongly influenced by the desired operating lift coefficient. Although wind turbine performance improves with high values of $L / D$, the corresponding high values of operating $C_{L}$ rapidly reduces the required $A F$ as shown on figure 4-3. Moreover, cost considerations of the transmission system lead to high design velocity ratios which require blades of low $\mathrm{AF}$. Accordingly, the selection of the airfoil type must be based on the maximum L/D consistent with practical blade geometries and high performance levels as discussed above in Section 4-2. From that discussion and a review of figures 4-3, an operating lift coefficient of 1.0 appears to be a good choice for two bladed wind turbines. Thus, in consideration of this operating $C_{I}$ level, the NACA $230 \mathrm{XX}$, the NACA $44 X X$ and the GA(W)-2 airfoil series were chosen for the parametric study. The NACA 230XX Series (ref. 12) was selected as the basic design because of the high $L / D$ levels extending cover a wide range of lift coefficients at Reynolds numbers appropriate to large wind turbines. Moreover, the section profiles incorporate no concavity which significantly eases fabrication when the very attractive, cost effective filament winding process is utilized. Nearly all of the other candidate airfoils exhibit concavity on the pressure surface. As a point of clarification, earlier data (ref. 16) for the NACA $230 \mathrm{XX}$ airfoil series used in some wind turbine analysis indicated reduced performance (fig. 4-25). The two sets of data presented in figure 4-25, were tested in different wind tunnel facilities. The data of reference 16 were obtained in an airfoil test facility which required corrections for: 1) conversion to free-air, 2) conversion to infinite aspect ratio, 3) support interference, 4) allowance for the flow at the tip of the rectangular airfoil, and 5) a correction to an effective Reynolds number. On the other hand, the data of reference 12 were obtained in a facility that required corrections for wall effect and constraint only. The reference 12 measurements are the more accurate and are generally recognized as being the standard against which other airfoils are compared.

It should also be noted that the stall characteristics of the NACA $230 \mathrm{XX}$ are more abrupt than those of the NACA 44XX and the GA(W) Series. However, aerodynamic analyses have shown that between start-up and rated wind velocities, wind turbines are designed to operate at angles of attack up to values well below the stall angle. For velocities greater than the rated, with variable pitch capability, the blade angle will be increased to maintain a constant output power and angles of attack will be below stall. Only at shutdown when the blades are driven to the feather position is stall encountered. However, in this case the effect of stall is unimportant.

The NACA $44 \mathrm{XX}$ airfoil series was included because it provides a higher $L / D$ and is less sensitive to surface roughness than the NACA 230XX Series. The Whitcomb GA(W) Series was included because of its relative insensitivity to surface roughness over the Reynolds number range for large wind turbines. Although the Wortmann and Liebeck airfoils show very high L/D's at high operating lift coefficients, these airfoils were not included in this study 
since it was judged that their true effectiveness could not have been demonstrated in the low scale of the test models.

From the power ratio plots presented in figure 4-26 the annual output power curves (figure 4-27) were derived. A study of these data indicates that at maximum performance, the annual energy output is increased $1.0 \%$ over the basic wind turbine by incorporating the NACA 44XX sections and reduced $2.0 \%$ by utilizing the Whitcomb GA(W) sections.

The wind turbine performance study described above is based on 3 million Reynolds number and smooth airfoil sections. The parametric variations discussed previously, with the exception of airfoil sections, are essentially independent of Reynolds number and roughness. Thus it is now appropriate to investigate the effect of Reynolds number and surface finish on wind turbines to complete the airfoil study. The effect of these parameters will be covered in the following text.

\subsubsection{Reynolds Number}

The wind turbine performance study described above is based on 3 million Reynolds number (RN) and smooth airfoil sections. For the RN range of 3 to 11 million which is representative of large wind turbines in the 30.561 meters (100-200 ft) diameter range, the parametric variations discussed previously are essentially independent of RN.

Since the test models cannot be tested at full scale RN, a study was made to determine the effect of RN for smooth sections on performance. The summary of the power ratio for the basic turbine is presented on figure 4-28 for a RN range of 0.7 to 6 million. It can be seen from figure 4-28 that the effect of $\mathrm{RN}$ above 3 million is negligible. Below this value, the power ratio decreases with increasing rate as $\mathrm{RN}$ is reduced to the minimum value. Consequently, the annual energy output and the rated kilowatts are correspondingly reduced with decreasing $\mathrm{RN}$ as shown in figure 4-29. A similar study was made for the basic wind turbine incorporating NACA 4412 sections and incorporating Whitcomb GA-W(2) sections. The corresponding percentage changes in annual energy output from the basic wind turbine with NACA 23012 sections are shown on figure 4-30.

As previously shown for 3 million RN, figure 4-30 indicates that over the complete range of RN investigated, wind turbine performances increases slightly with the NACA 4412 section and decreases slightly with the Whitcomb GA(W) sections compared to that for the basic wind turbine incorporating NACA 23012 sections.

A 2.44 meter ( $\mathrm{ft}$ ) diameter was chosen for the model wind turbines because it is the largest diameter compatible with existing test hardware and the

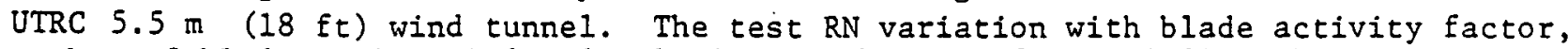
number of blades, tip speed and velocity is shown on figure 4-31. The models have been designed for a maximum tip speed of $700 \mathrm{fps}$ to avoid compressibility losses. The test RN range will be from .1 to 1.1 million as is shown on figure 4-31. Therefore, the performance of the model wind turbines is expected to be significantly less than that of full scale wind. turbines where RN range from 3 to 9 million representative of 23-61M (75-200 $f t$ ) diameters. The expected performance levels of the models will be calculated later in this program as discussed in Section 9. 


\subsubsection{Roughness}

The effects of blade surface roughness on performance has always been of concern for propellers and helicopter rotors. NACA (ref. 12) had established a procedure for simulating roughness by applying number 60 grit wrapped the leading edge on both surfaces back to 0.08 chord. More recently NASA has revised the roughness tests according to reference 17. Now, commercial grit number 120 is applied at 6 million RN and grit number 60 at 3 million in transition strips $0.25 \mathrm{~cm}(0.10 \mathrm{in})$ wide. The roughness is sparsely spaced and attached to the airfoil surface with lacquer. The more recently adapted NASA roughness criteria is less severe than the one previously used by NACA.

For the airfoil variation discussed previously, a roughness study was made based on the NACA criteria because of available airfoil data. The reductions in performance due to roughness are shown for the basic turbine (NACA 23012 sections) in figure 4-32 showing the effect on power ratio and figure 4-33 shows the effect on the rated kilowatts and annual energy output. From figure 4-33 it can be shown that roughness reduces the performance of the basic turbine with NACA 23012 sections approximately $10 \%$ below that of the smooth section over the entire Reynolds number range. For the turbine with NACA 4412 sections, the roughness reduced performance $6 \%$ over the corresponding smooth sections while the similar number of the Whitcomb GA(W)-2 is $4 \%$. However, although the roughness effect is the least with the GA(W) -2 airfoil sections, the performance of the turbines with the NACA 4412 sections is slightly less. These conclusions are sumnarized in figure 4-30.

The roughness criteria discussed above is based upon propeller and helicopter rotor experience where most surface erosion is due to stone and gravel damage picked up from the ground during take-off. Since most large wind turbines will be placed relatively high above the ground where the stone and gravel pick up will be negligible, it is safe to conclude that the erosion damage will be less severe than that considered above. In this regard it is interesting to note that Professor Hutter indicated that his blades showed no deterioration in performance after many hours of running.

\subsubsection{Waviness}

Since surface waviness may be encountered in the manufacturing of turbine blades, its effect on performance should be considered. Waviness, if sufficiently large to effect the chordwise pressure distribution such that laminar separation occurs, may result in premature transition with a deteriorating effect on airfoil performance characteristics.

An example of the effect of waviness on airfoil performance characteristics is presented in reference 18. Waviness was simulated by skin wrinkles on a NACA 66, 1-115 and a NACA 23015 airfoil sections. The wave had a height/ chord ratio of .0011 and a length/chord ratio of .06 . Although the normal force (lift) and the pitching moment were not appreciable changed, the L/D was reduced approximately thirty percent from that for a smooth section. As a point of reference, the $L / D$ was reduced approximately fifty percent for a NACA 23015 section with NACA roughness.- Thus the simulated waviness incorporated in the above mentioned airfoils have less adverse effect on wind turbine performance than that predicted for NACA roughness. It is expected that if waviness is eliminated over approximately twenty-five percent of the leading edge, the effect on drag should become negligible. 
The wave height, shape and number per unit length must be considered in evaluating the effect on performance. Due to insufficient experimental data, it was not possible to include the effect of waviness on wind turbine performance in this study. However in view of possible waviness occuring on the blade surface within realistic tolerances of the more cost effective, candidate fabrication techniques, its effect on performance will be investigated as part of the wind tunnel program.

\subsubsection{Blade Root Cut-off}

Blade root cut-off is of interest as a potential for reducing costs since a spar is less costly than blading. Therefore, the effect on performance of progressively reducing blading as shown on figure 4-34 was investigated. The reduction in power ratio is due to losses associated with the reduced blade area and the drag of the exposed spar. The power ratio data is shown in figure 4-35 and the annual output in figure 4-36. It can be seen from figure 4-36 that the reductions in annual energy output are as follows:

\section{Blade Root Cut-off}

$10 \%$ (Reference)
$25 \%$
$35 \%$
$50 \%$

\section{$\%$ Reduction Performance}

$0 \%$
$8 \%$
$19 \%$
$42 \%$

Thus it can be seen that there is a significant penalty in performance when the blade root cut-off is increased.

\subsubsection{Summary}

In summary, it is pertinent to briefly summarize the results of the parameter study in Table 4-1. The effect of the parameters discussed previously on annual energy output is presented in Table 4-1 in terms of percent change in annual energy output relative to the basix 2 blade/57.9 m (190 ft.) diameter/30AF/ trapezoidal planform/NACA 230XX airfoils wind turbine.

It is interesting to note that only increasing number of blades and airfoil type resulted in an improvement in annual energy output over the reference wind turbine. It is predicted that the largest losses will occur for a one bladed turbine. Large deviations from the optimum configurations in planform distribution, thickness distribution, and twist distribution will result is significant losses. Root cut-off beyond $15 \%$ will also result. in large losses. The model wind turbine test data are expected to show lower performance than that predicted for full scale wind turbines because of the limitations in test RN. Although the calculations indicate that losses due to roughness may be significant, as indicated previously, it is expected that these results are pessimistic. Similarly, waviness is expected to have some adverse effect on performance. However, because of insufficient data, it was not possible to analytically evaluate this effect. Accordingly, the effects on wind turbine performance of RN, surface roughness and waviness will be investigated in the wind tunnel as well as the prime, geometric parameters discussed in Section 6.0 . 
TABLE 1

EFFECT OF BLADE SHAPE PARAMETERS ON ANNUAL ENERGY OUTPUT

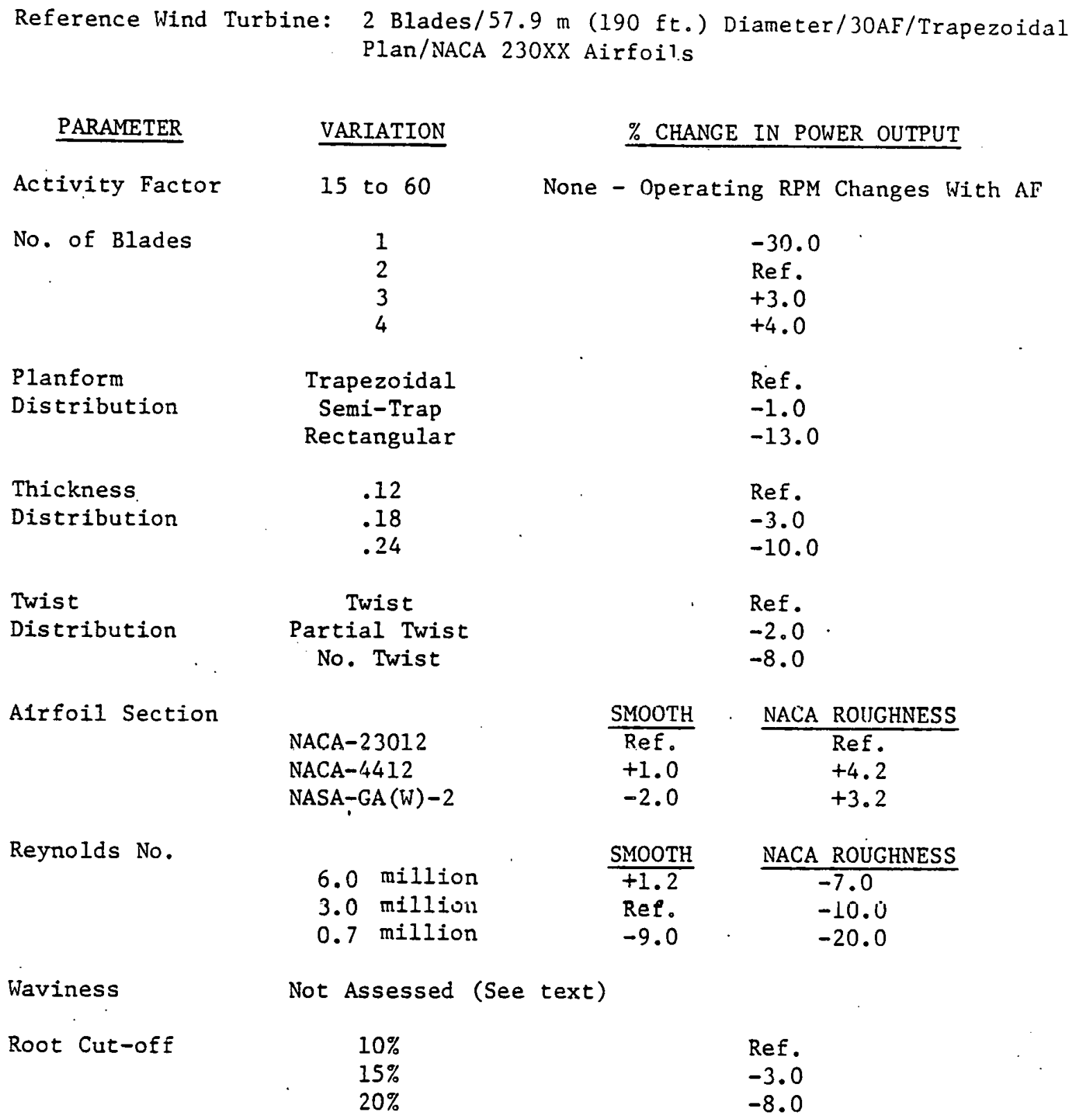




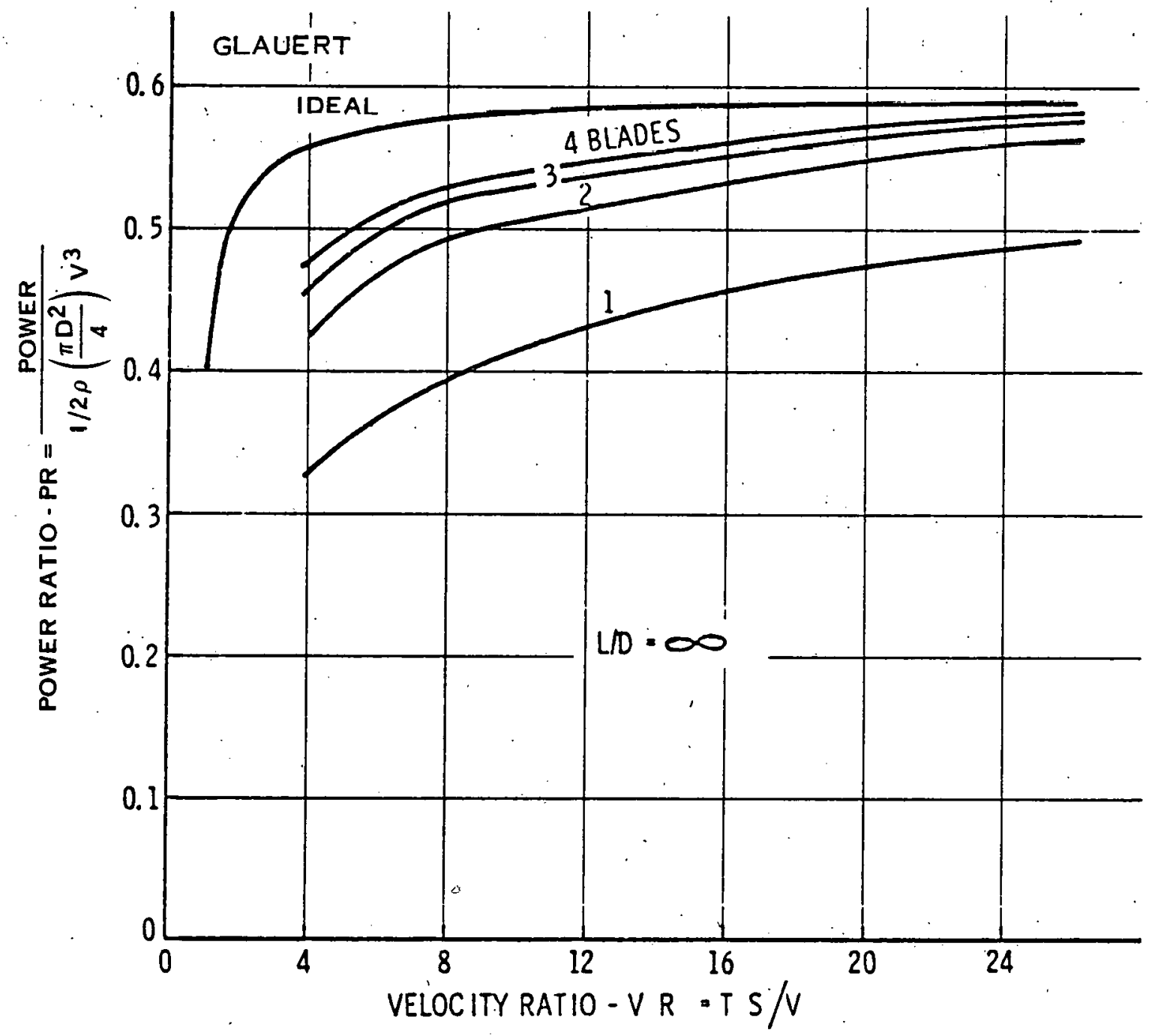

FIGURE 4-1. EFFECT OF NUMBER OF BLADES ON PEAK PERFORMANCE OF OPTIMUM WIND TURBINES 


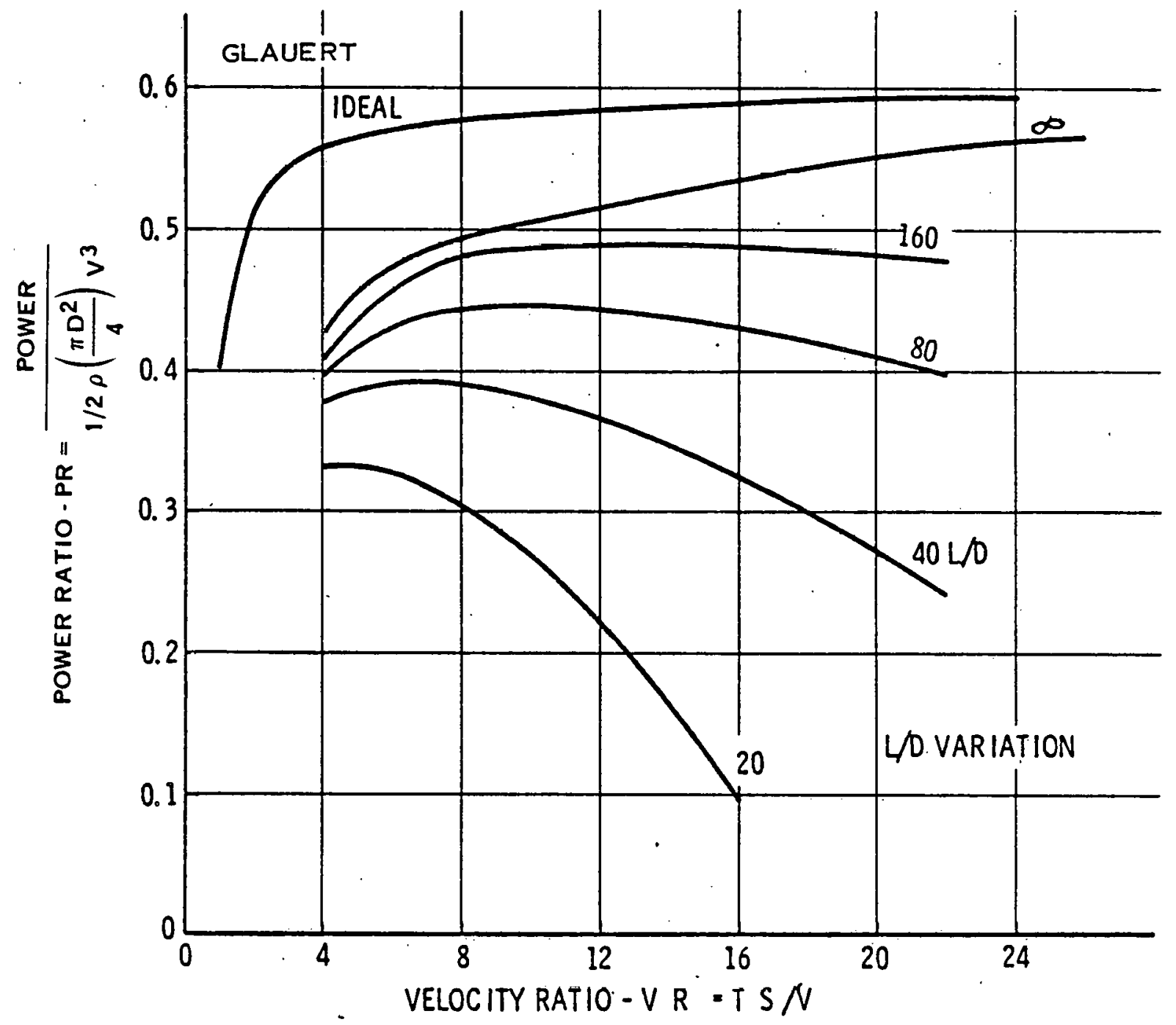

FIGURE 4-2. EFFECT OF L/D ON PEAK PERFORMANCE OF OPTIMUM, 2-BLADED WIND TURBINES 

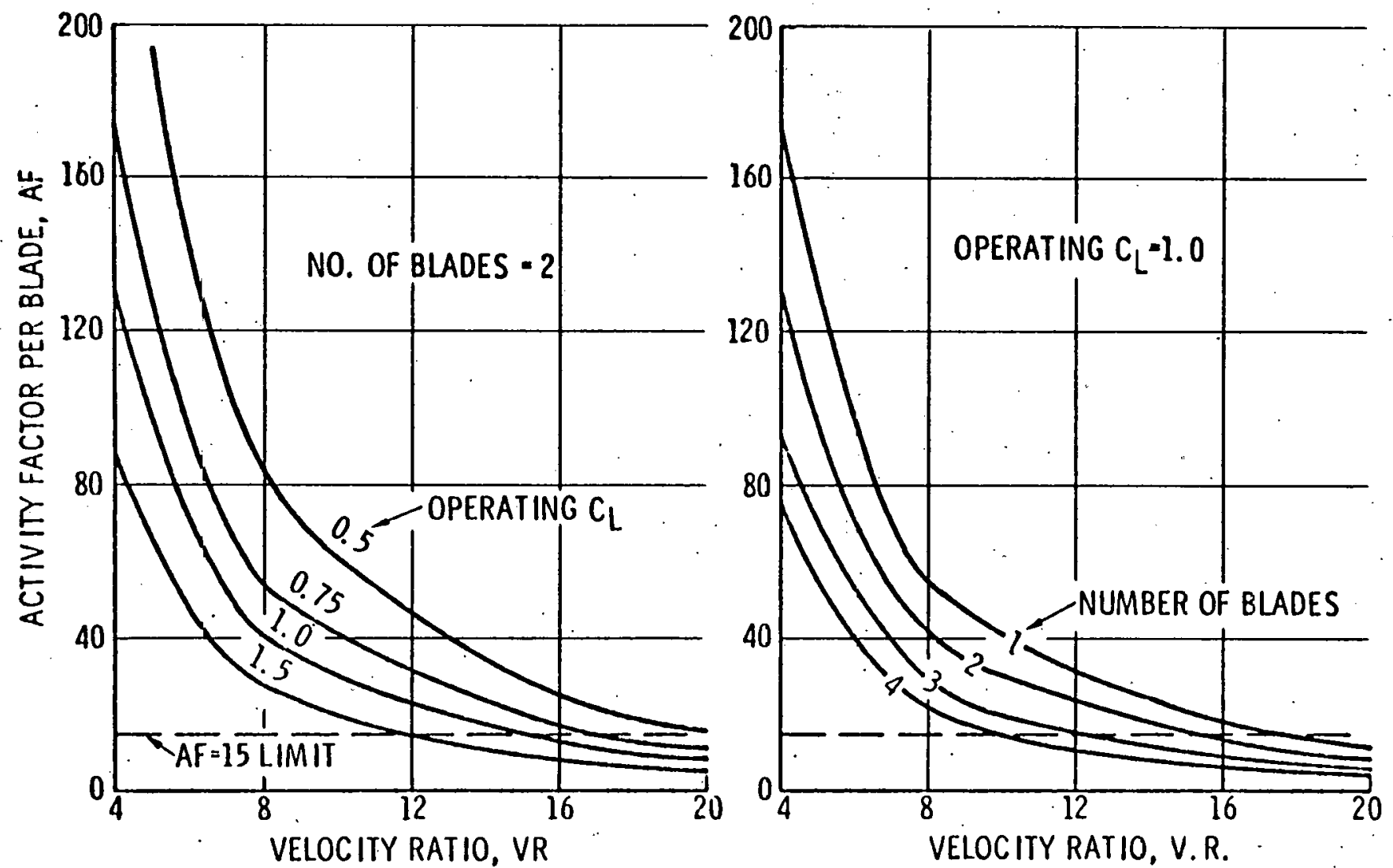

FIGURE 4-3. EFFECT OF VELOCITY RATIO ON ACTIVITY FACTOR OF OPTIMUM WIND TURBINES 


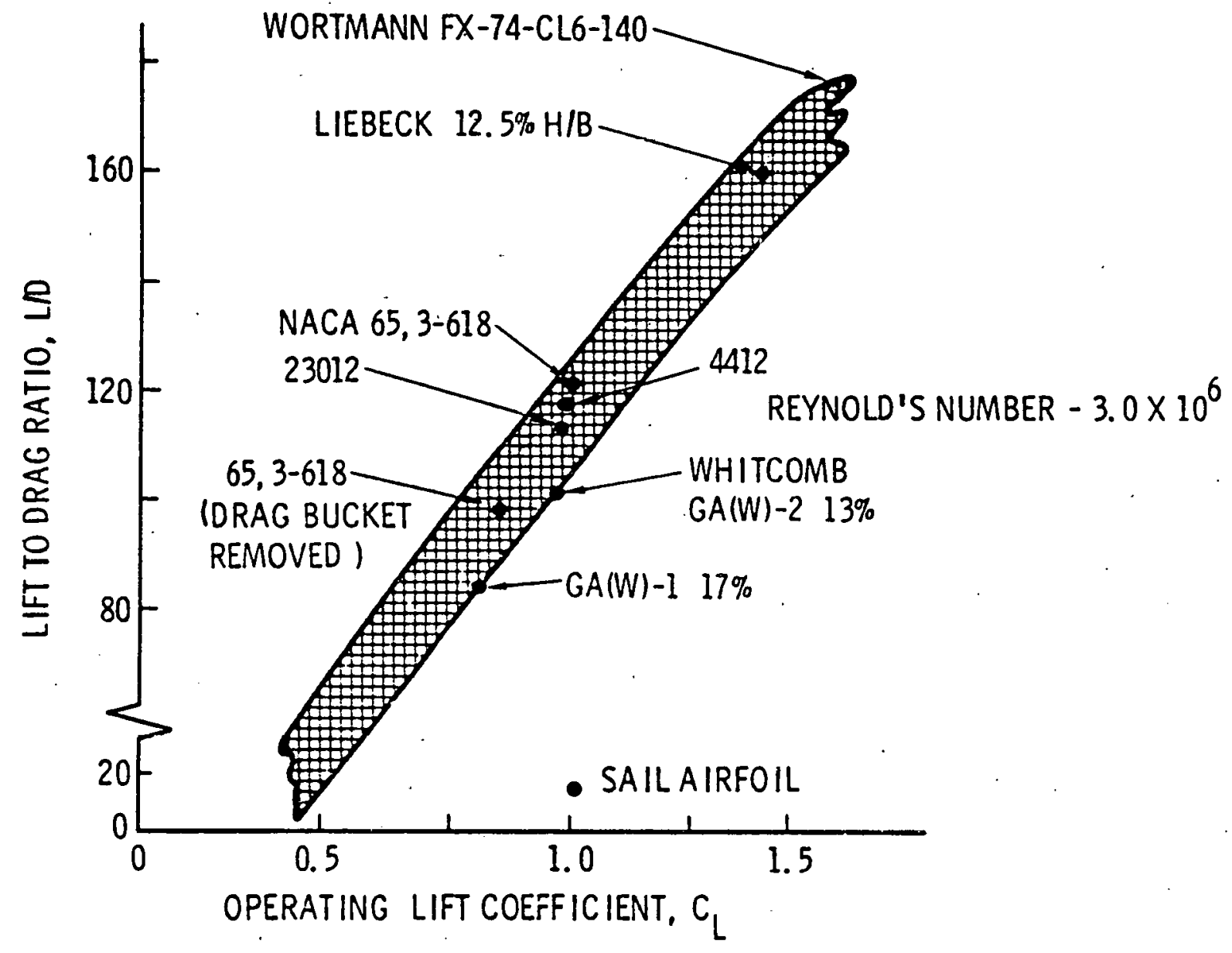

FIGURE 4-4. AIRFOIL SECTION LIFT-TO-DRAG RATIO TREND WITH OPERATING LIFT COEFFICIENT 


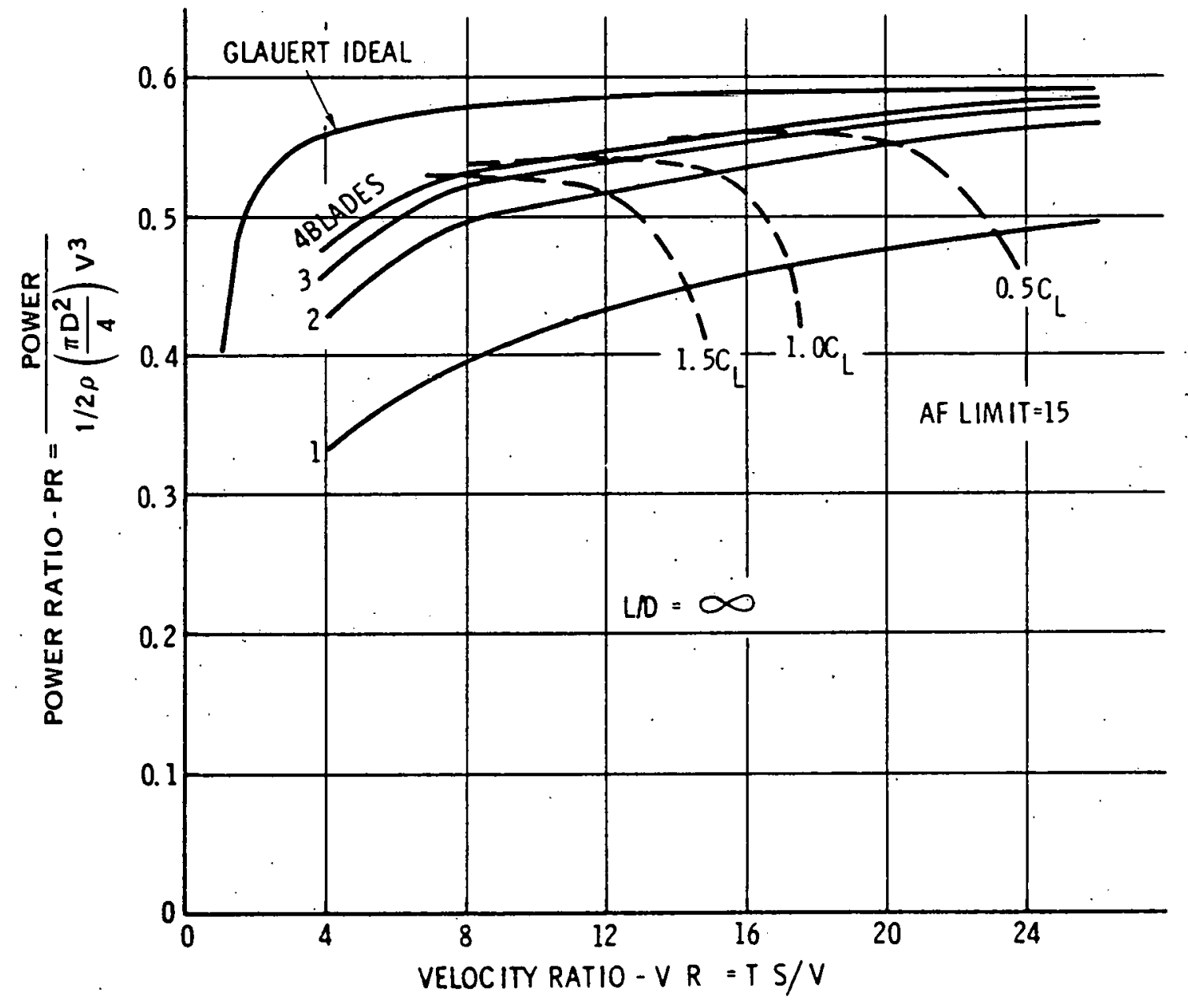

FIGURE 4-5. CONSTRAINT OF ACTIVITY FACTOR LIMIT ON PERFORMANCE SPECTRUM OF OPTIMUM WIND TURBINES 


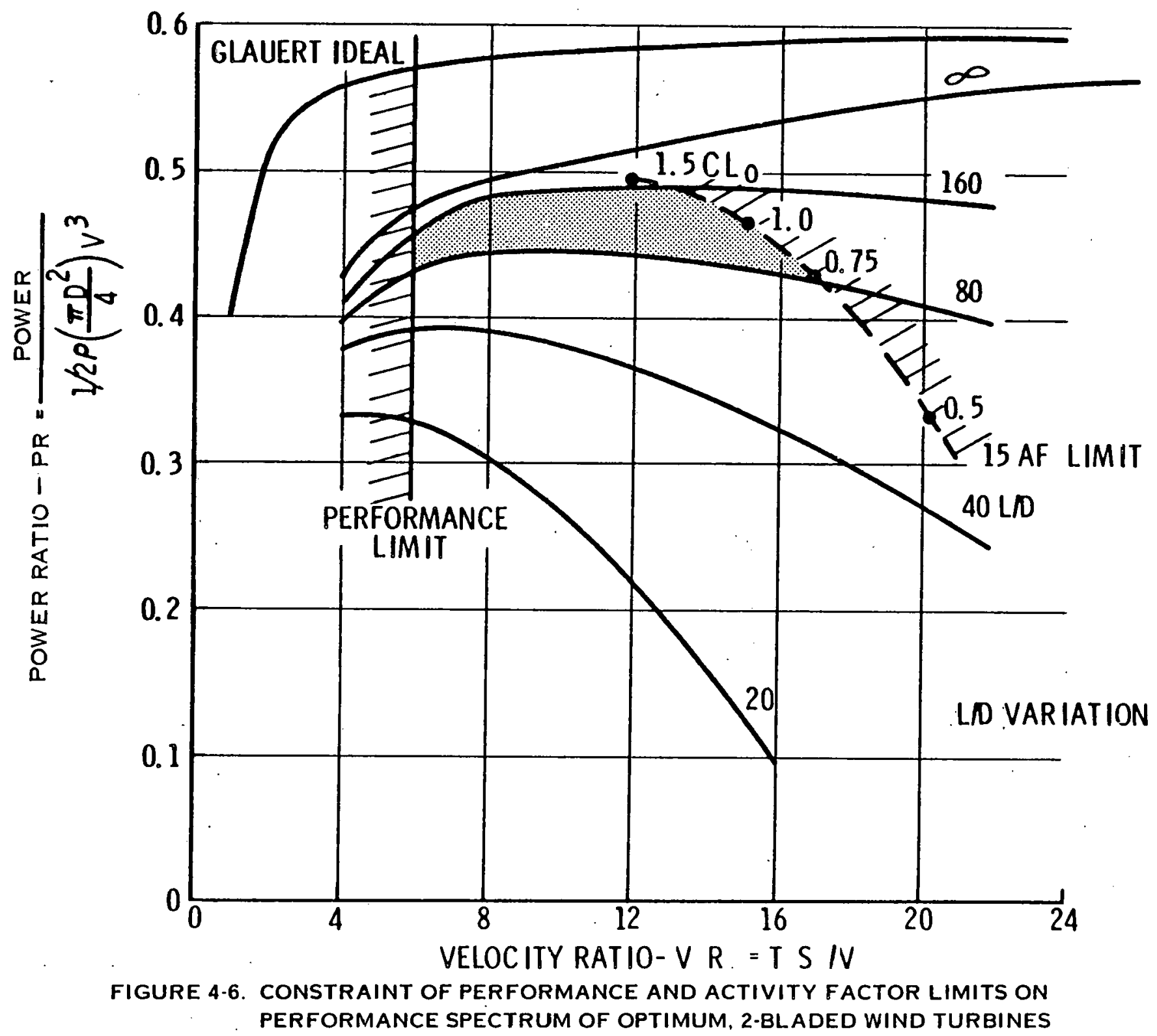



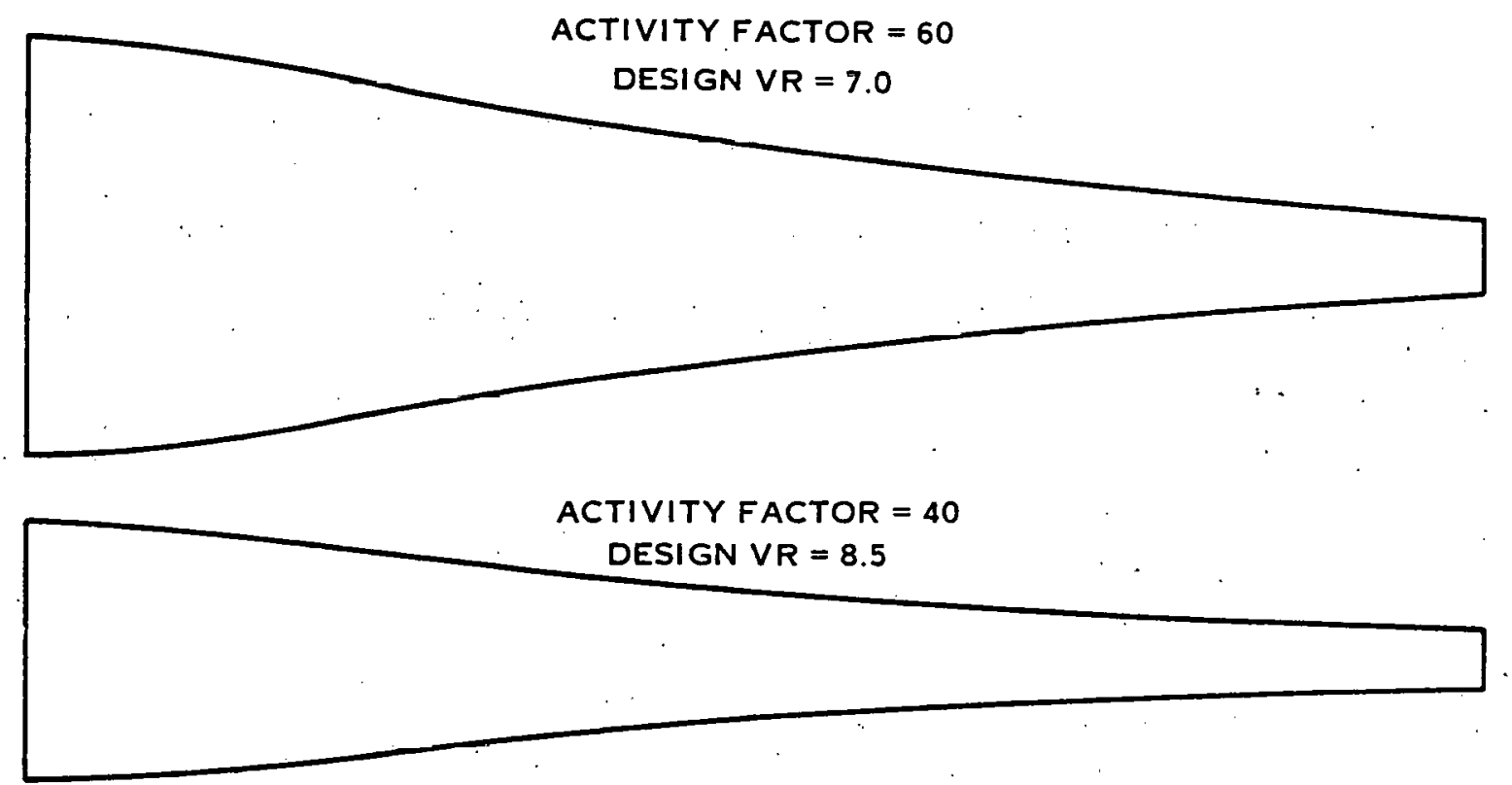

ACTIVITY FACTOR $=30$

DESIGN VR $=10$

ACTIVITY FACTOR $=20$

DESIGN VR $=12$

ACTIVITY FACTOR $=15$

DESIGN VR $=15.0$

FIGURE 4-7. BLADE PLANFORMS 


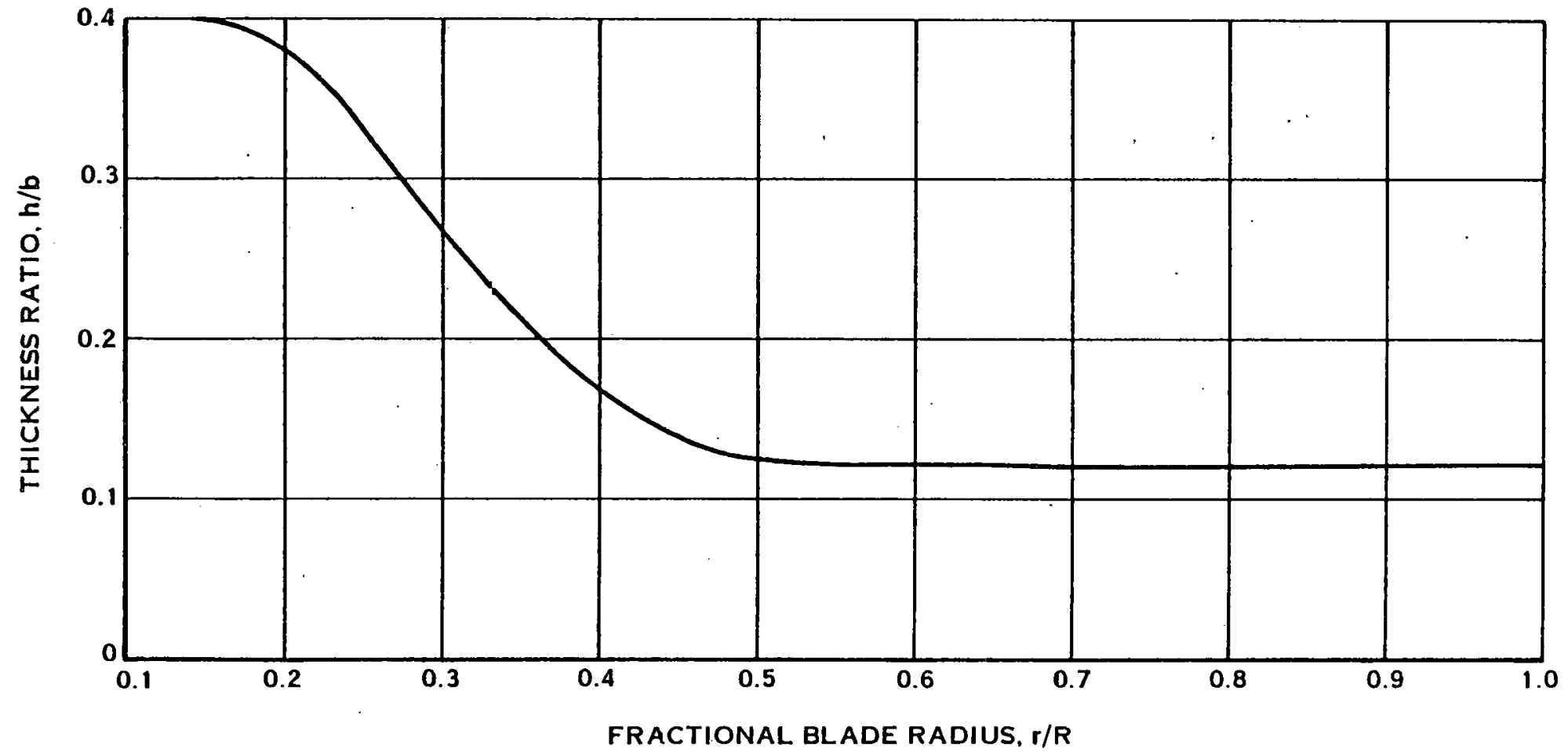

FIGURE 4-8. THICKNESS DISTRIBUTION 


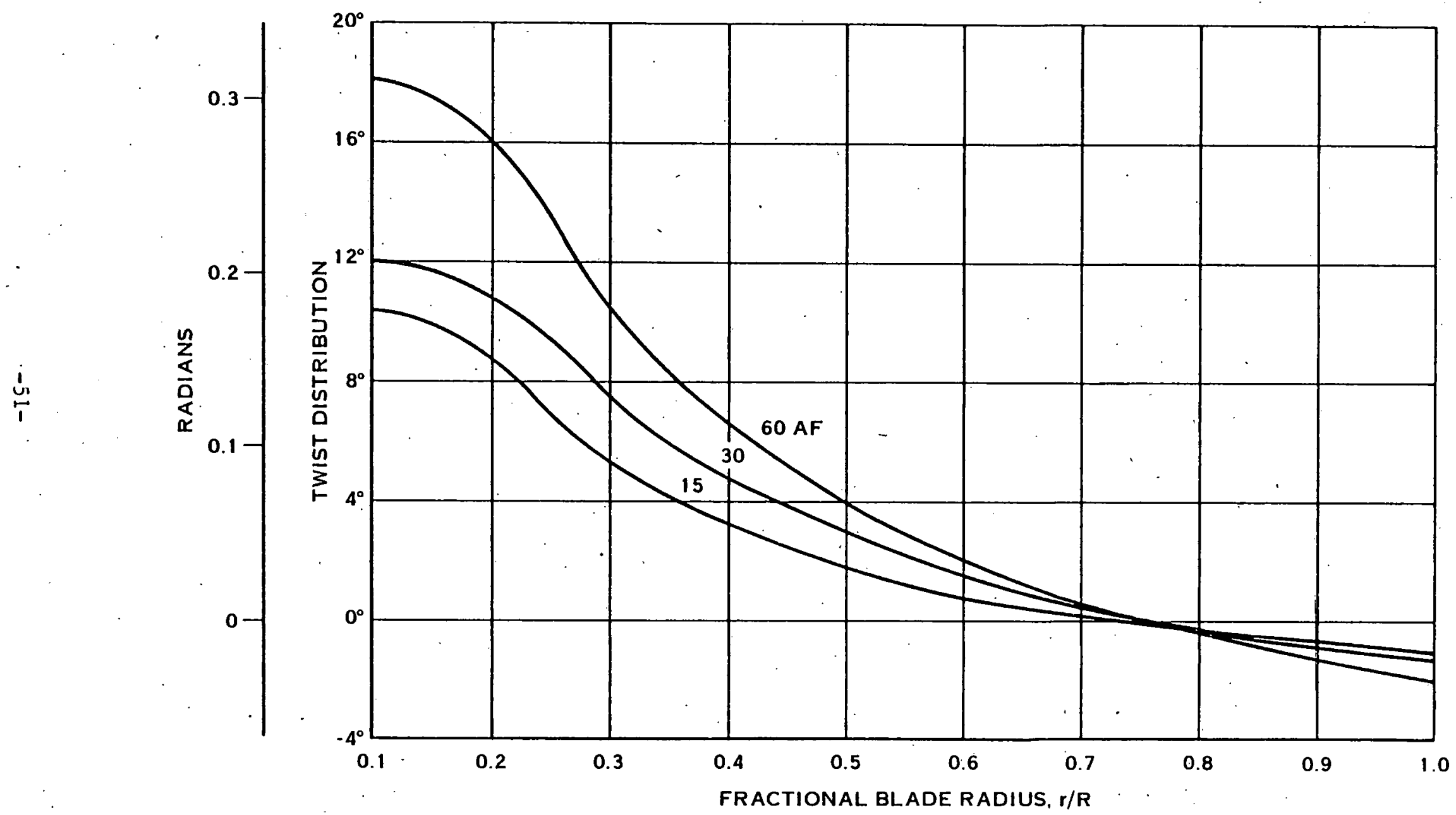

FIGURE 4-9. TWIST DISTRIBUTION 


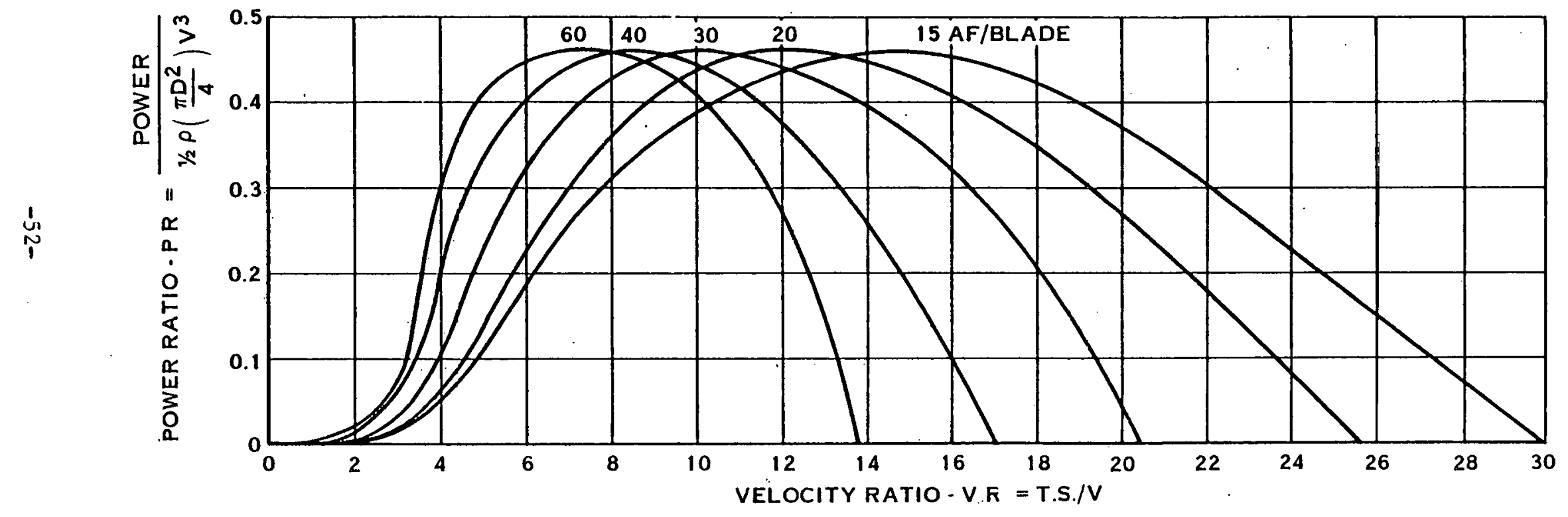

FIGURE 4-10. EFFECT OF ACTIVITY FACTOR ON THE POWER RATIO OF NEAR OPTIMUM 2-BLADED WIND TURBINES 


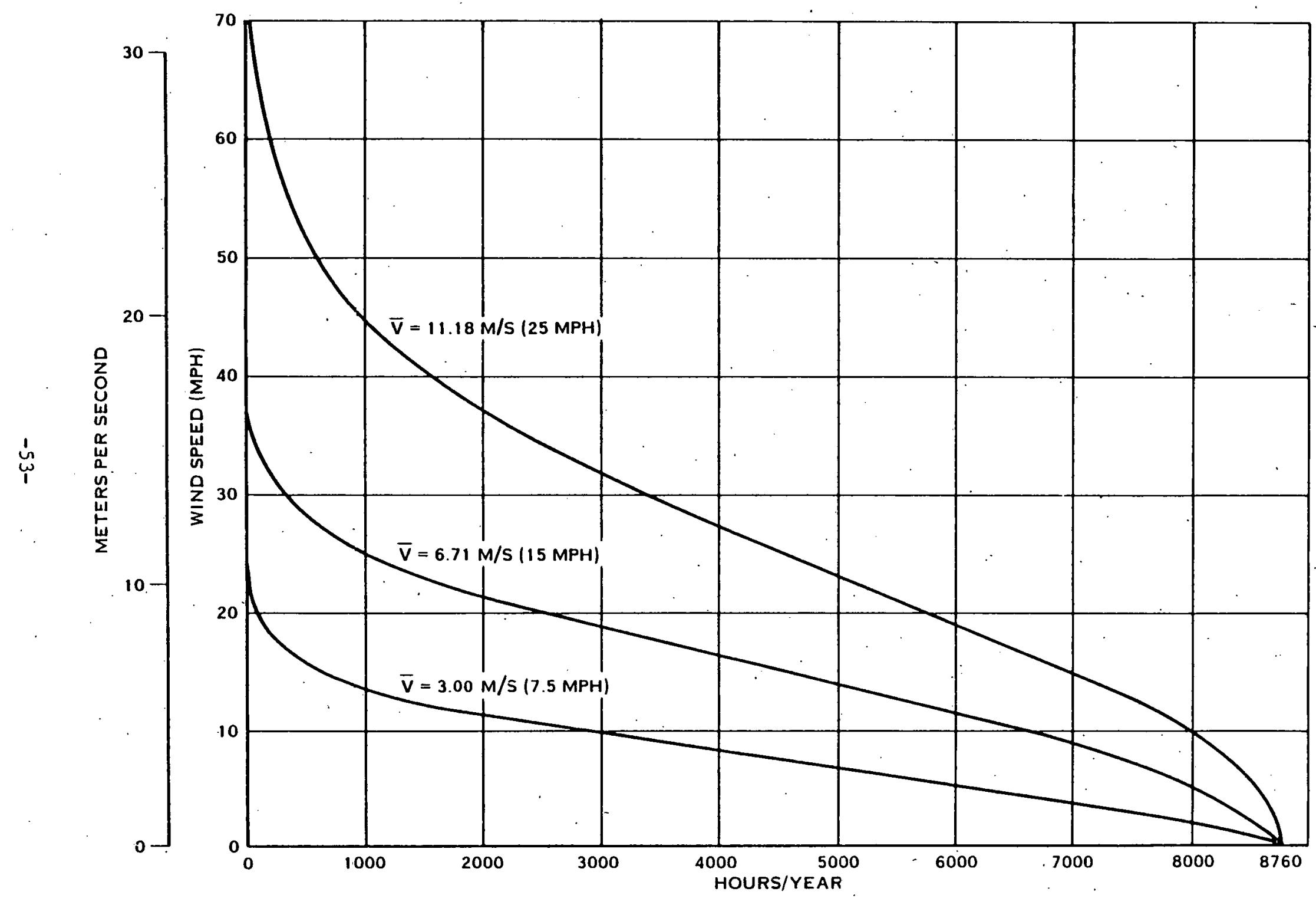

FIGURE 4-11. TYPICAL VELOCITY DURATION CURVES FOR VARIOUS MEAN WIND SPEEDS 


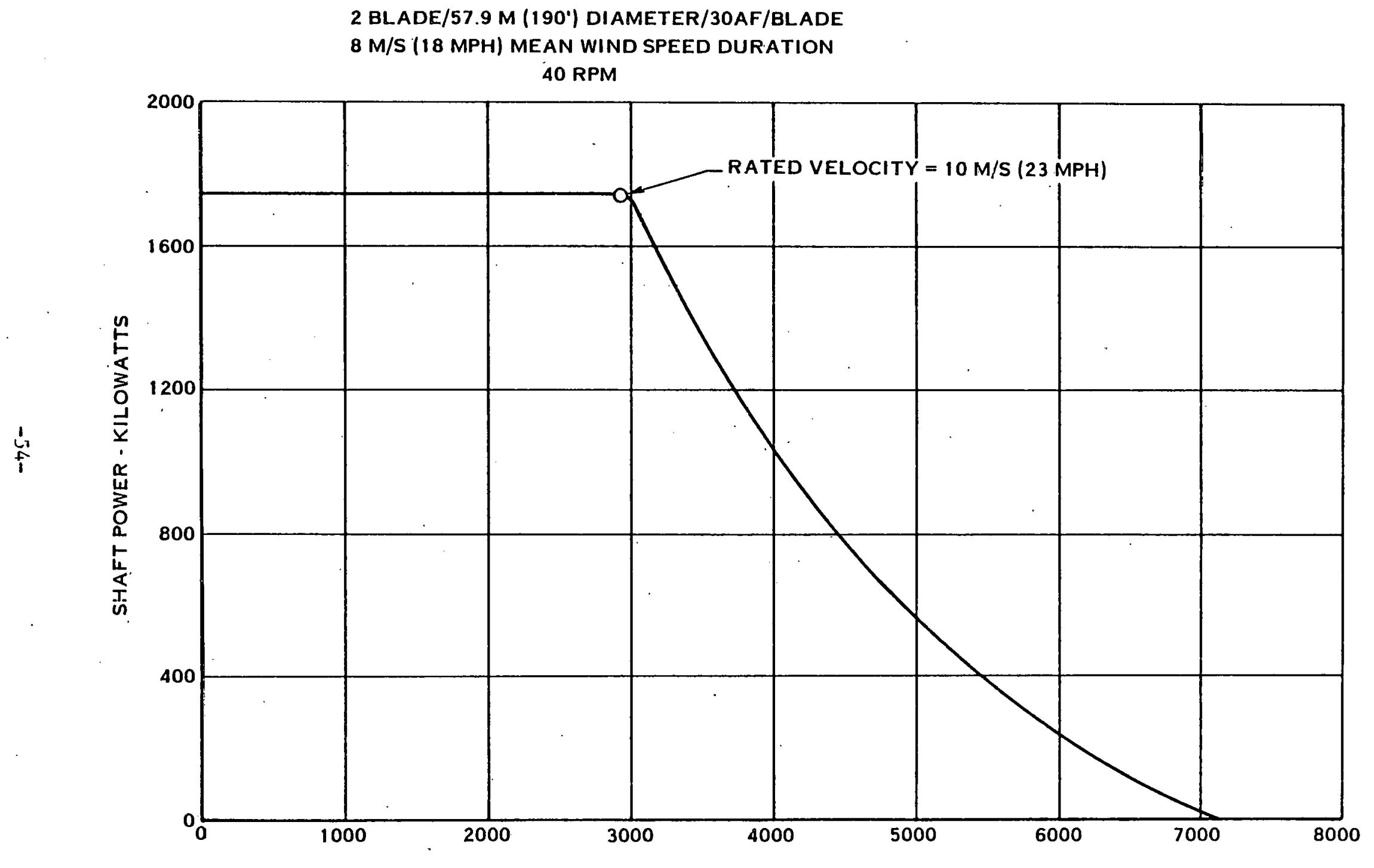

FIGURE 4-12. KILOWATTS VERSUS HOURS OF THE YEAR 


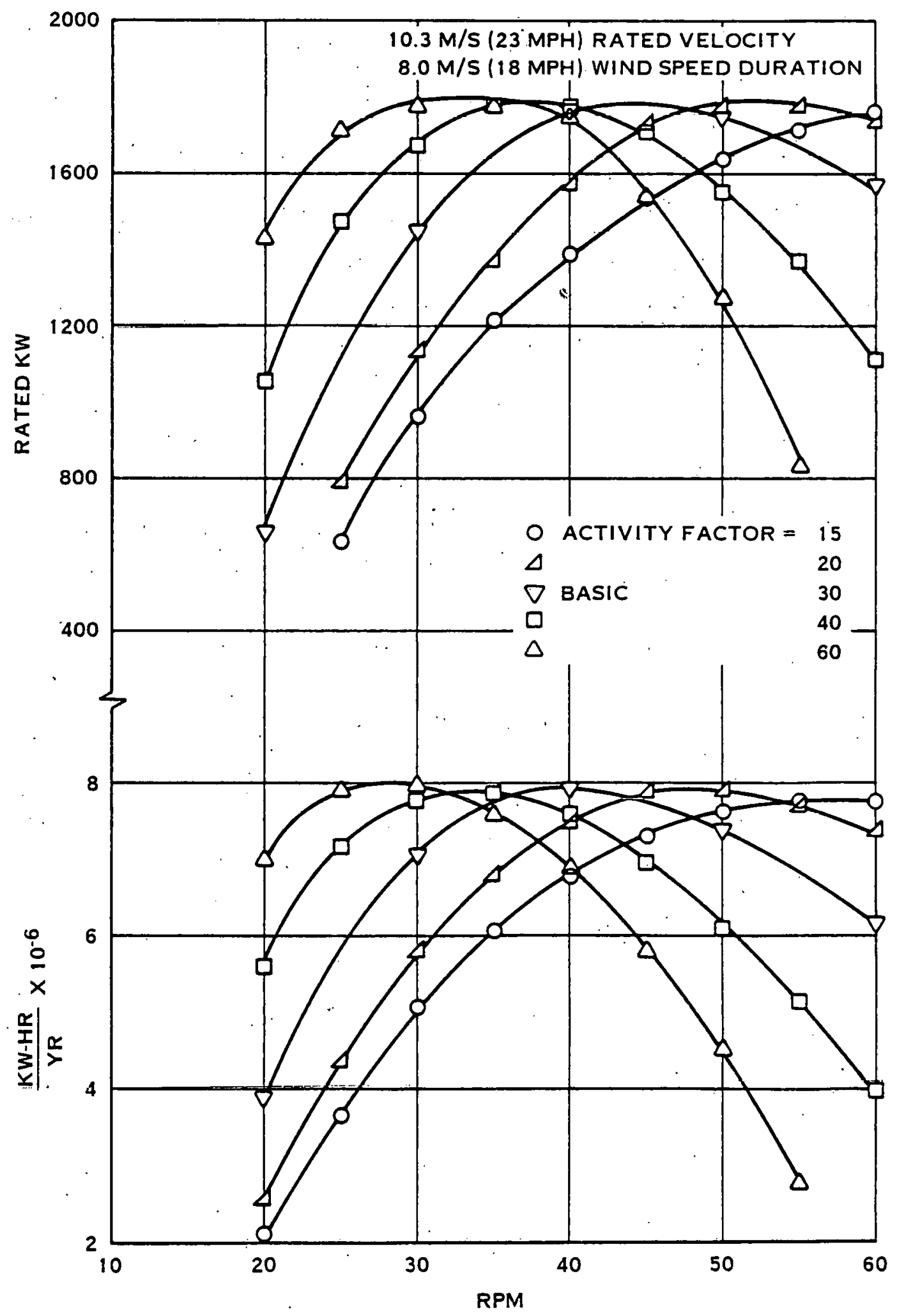

FIGURE 4-13. EFFECT OF ACTIVITY TACTOR ON THE ANNUAL ENERGY OUTPUT OF 57.9M (190 FOOT) DIAMETER, 2-BLADED WIND TURBINES 
30 ACTIVITY FACTOR

NACA 230XX AIRFOIL SECTIONS

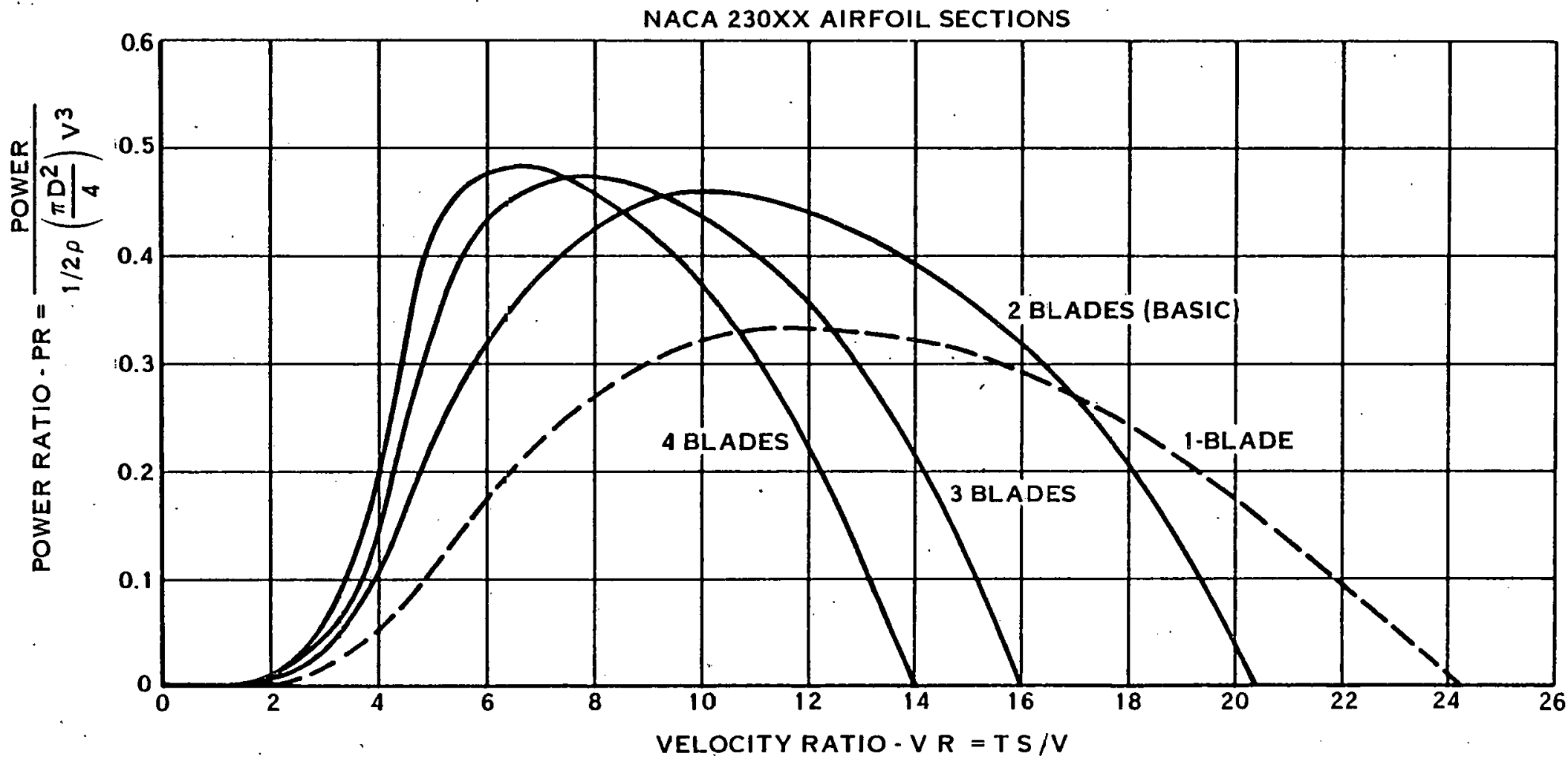

FIGURE 4-14. EFFECT OF NUMBER OF BLADES ON THE POWER RATIO OF NEAR OPTIMUM, 2-BLADED WIND TURBINES 


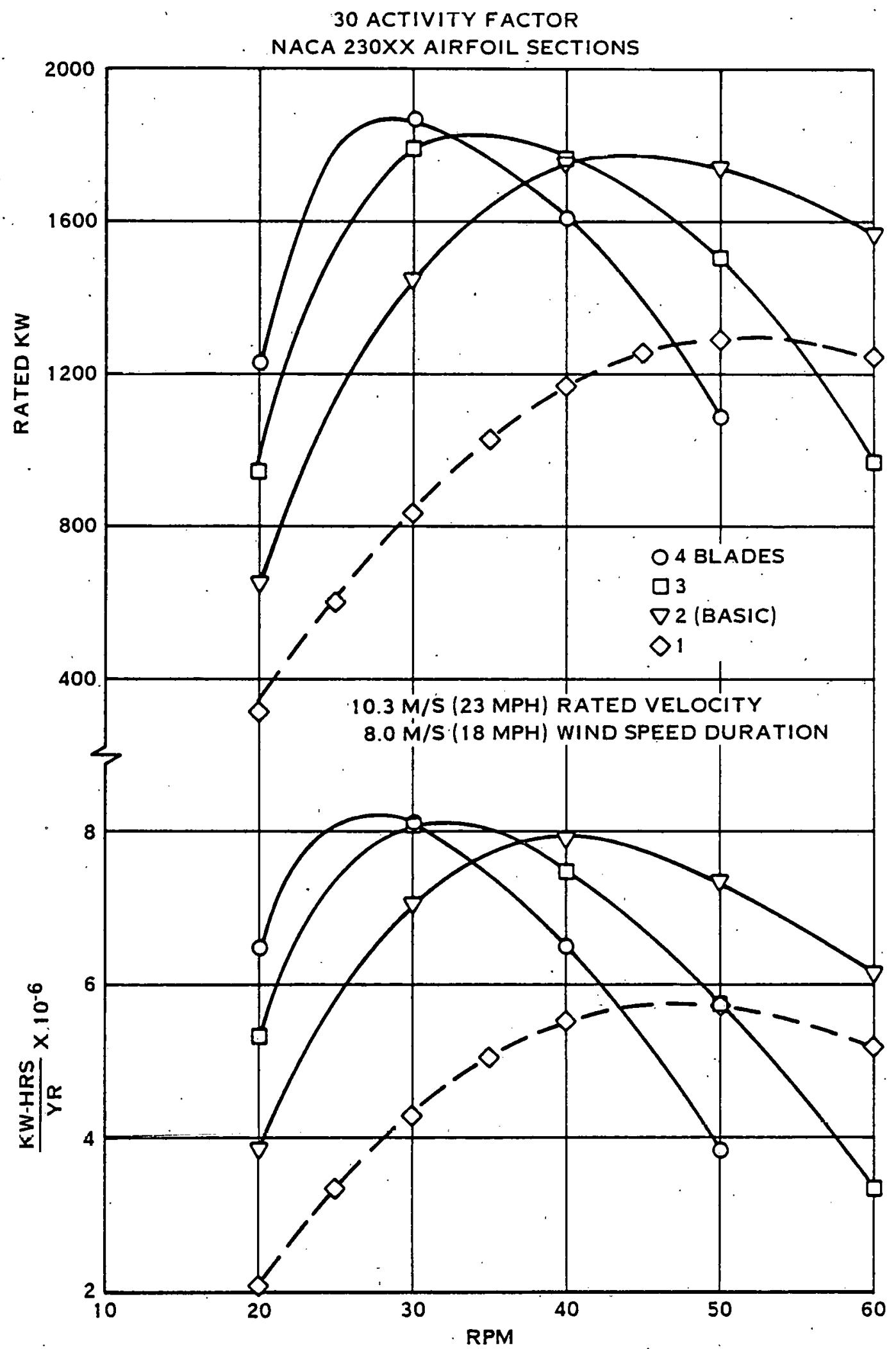

FIGURE 4-15. EFFECT OF NUMBER OF BLADES ON THE ANNUAL ENERGY OUTPUT OF $57.9 \mathrm{M}$ (190-FOOT) DIAMETER,2-BLADED WIND TURBINES 
30 ACTIVITY FACTOR

NACA 230XX AIRFOIL SECTIONS

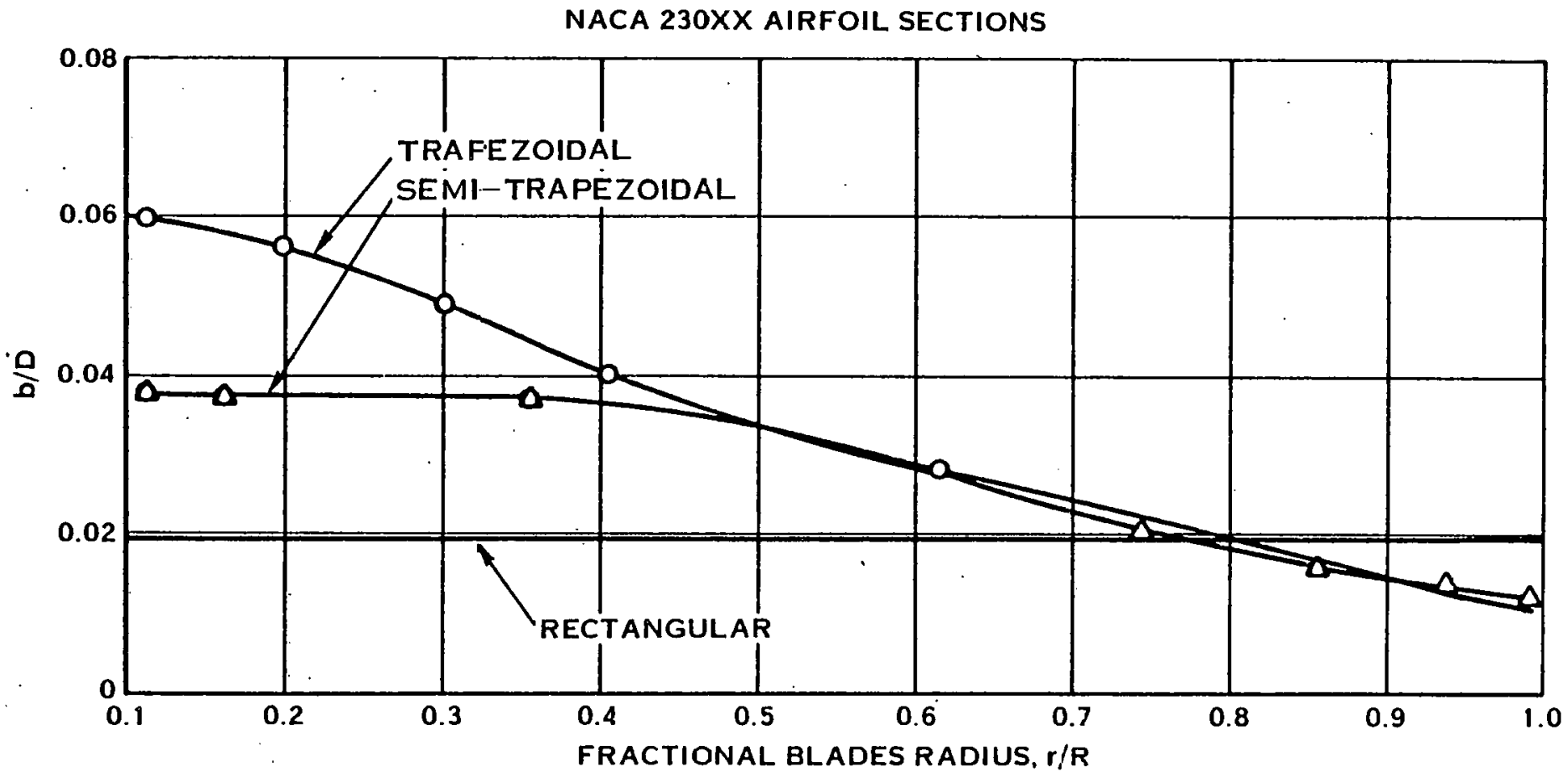

FIGURE 4-16. WIDTH VARIATIONS 


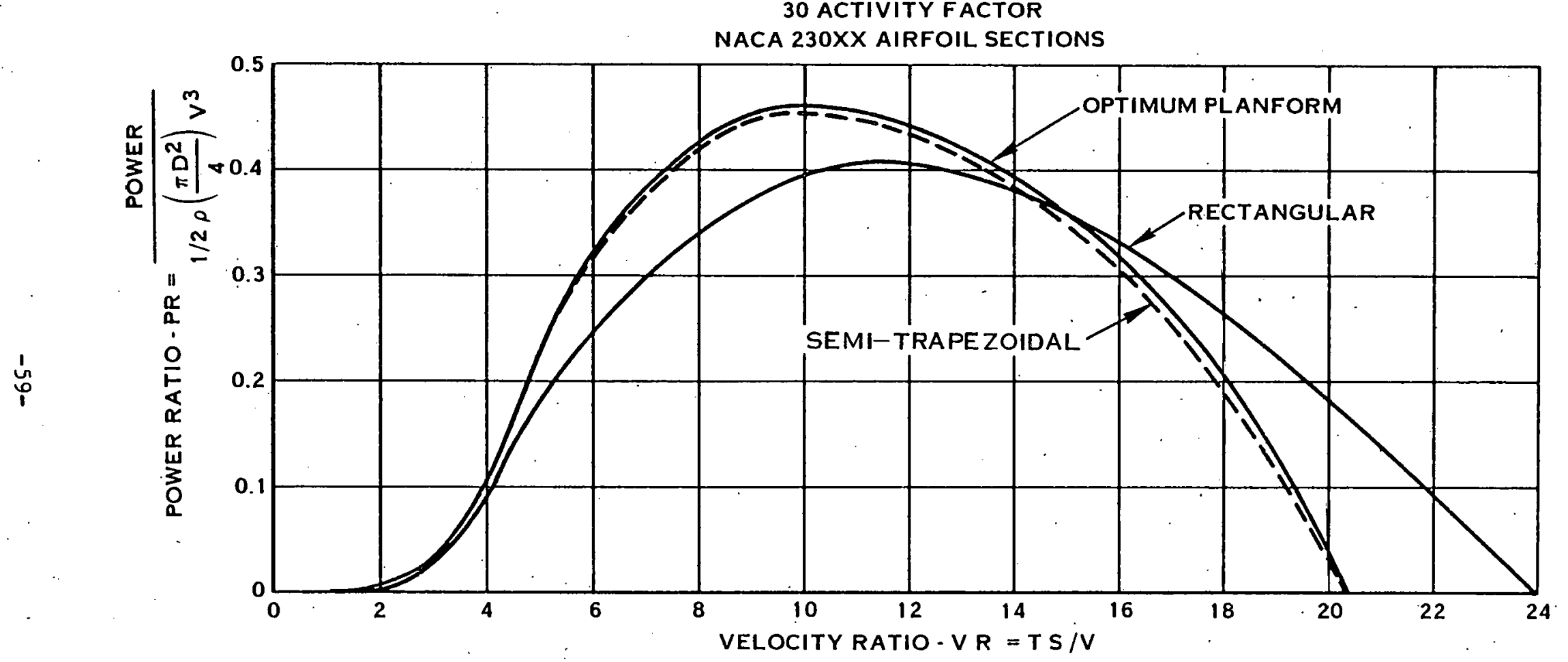

FIGURE 4-17. EFFECT OF PLANFORM VARIATIONS 


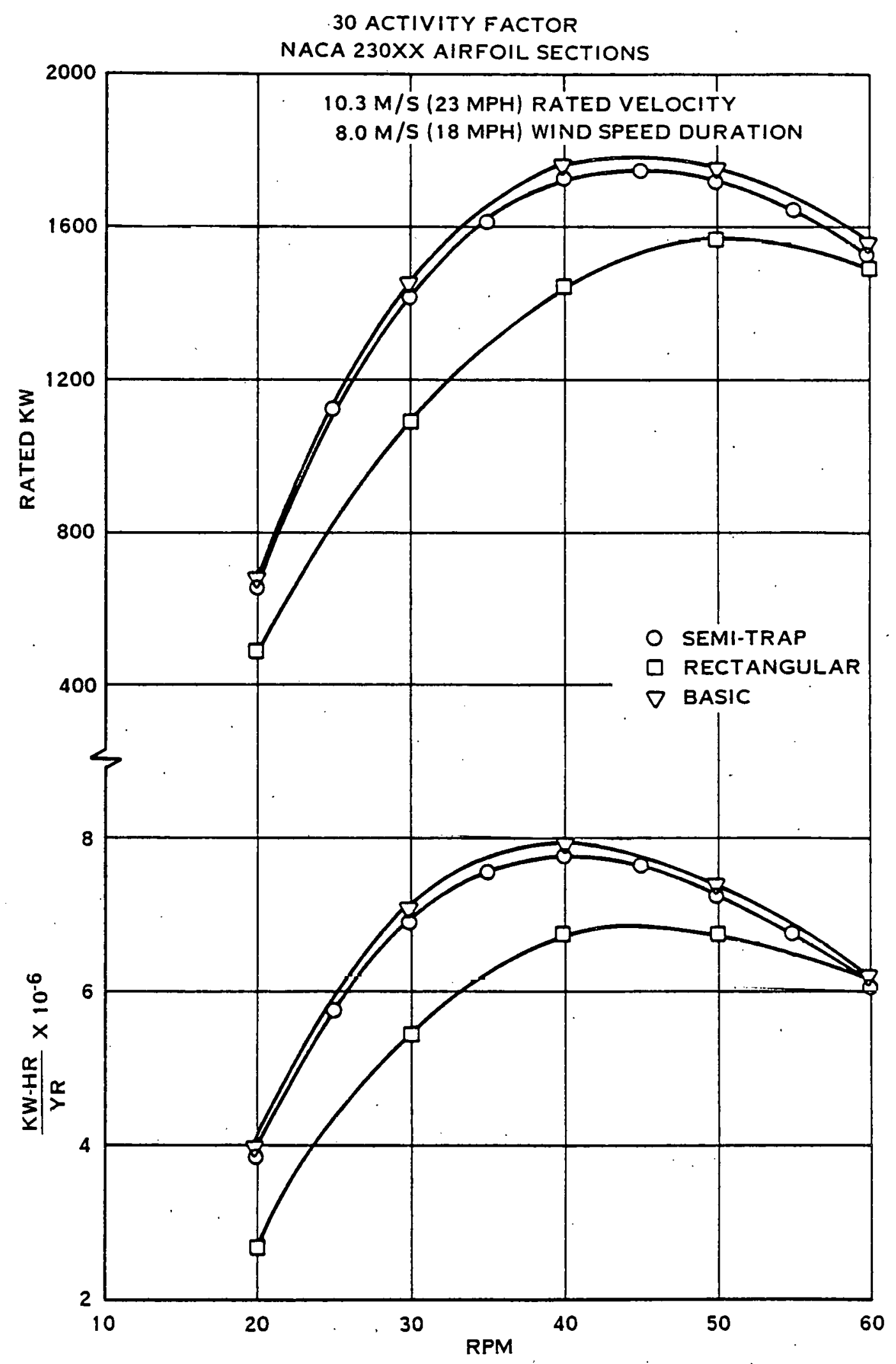

FIGURE 4-18. EFFECT OF PLANFORM ON ANNUAL ENERGY OUTPUT OF $57.9 \mathrm{M}$ (190-FOOT) DIA.METER,2-BLADED WIND TUR3INES 


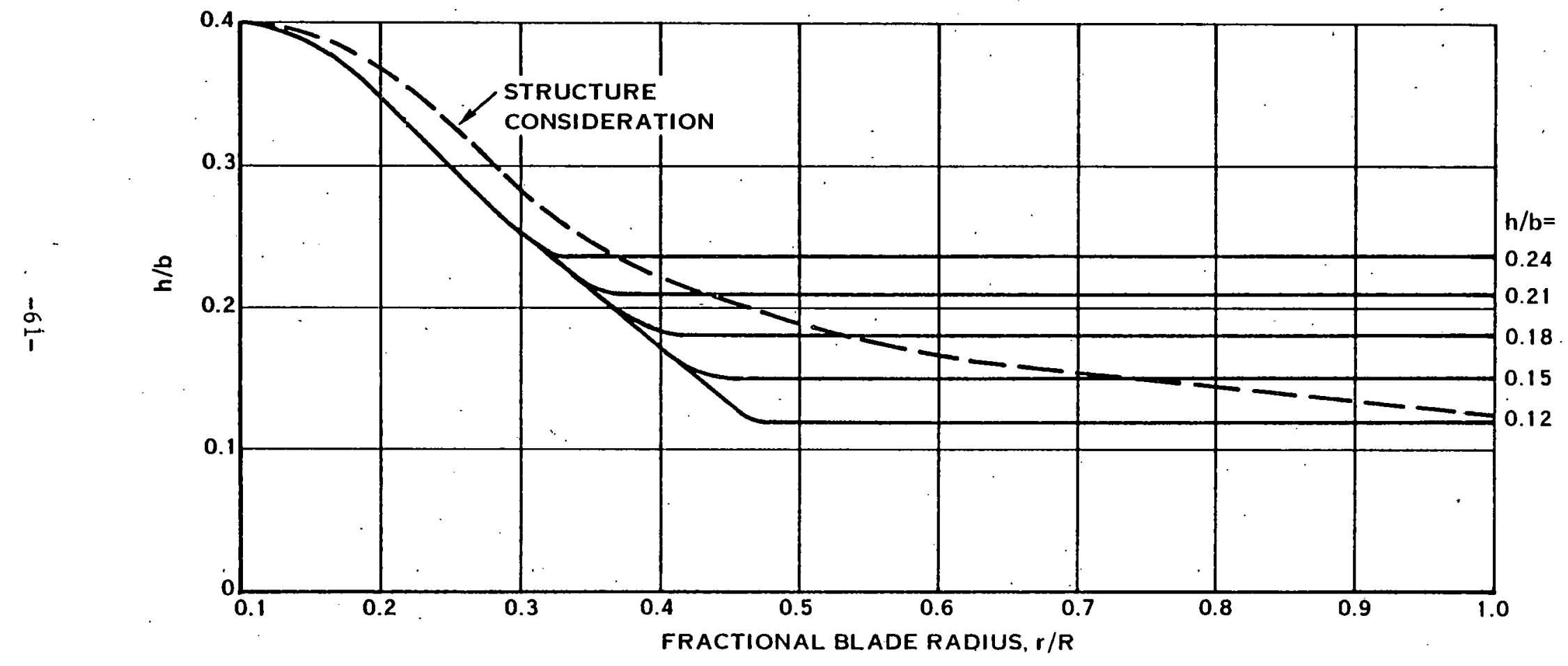

FIGURE 4-19. THICKNESS VARIATIONS 


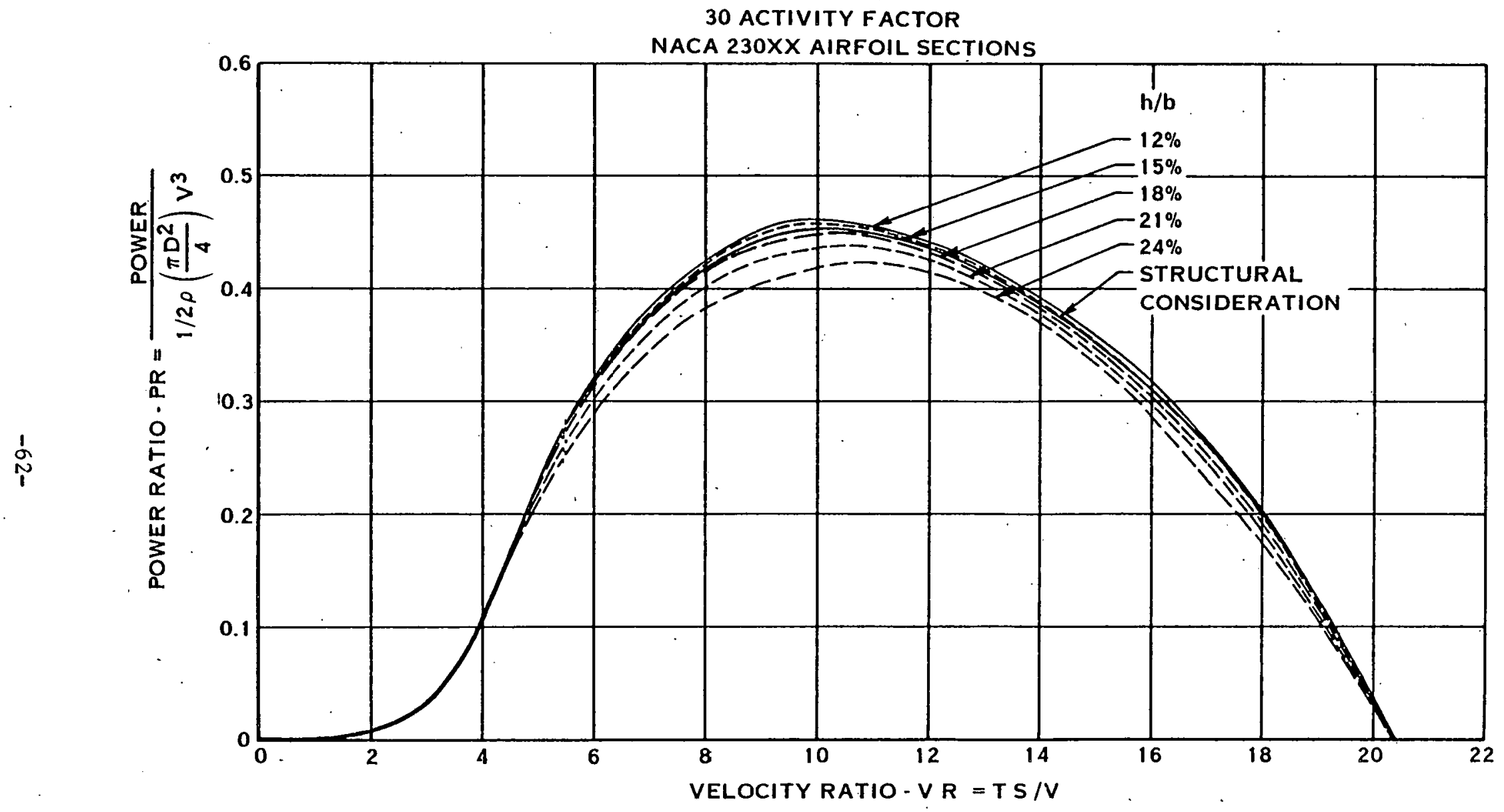

FIGURE 4-20. EFFECT OF THICKNESS DISTRIBUTION 
30 ACTIVITY FACTOR

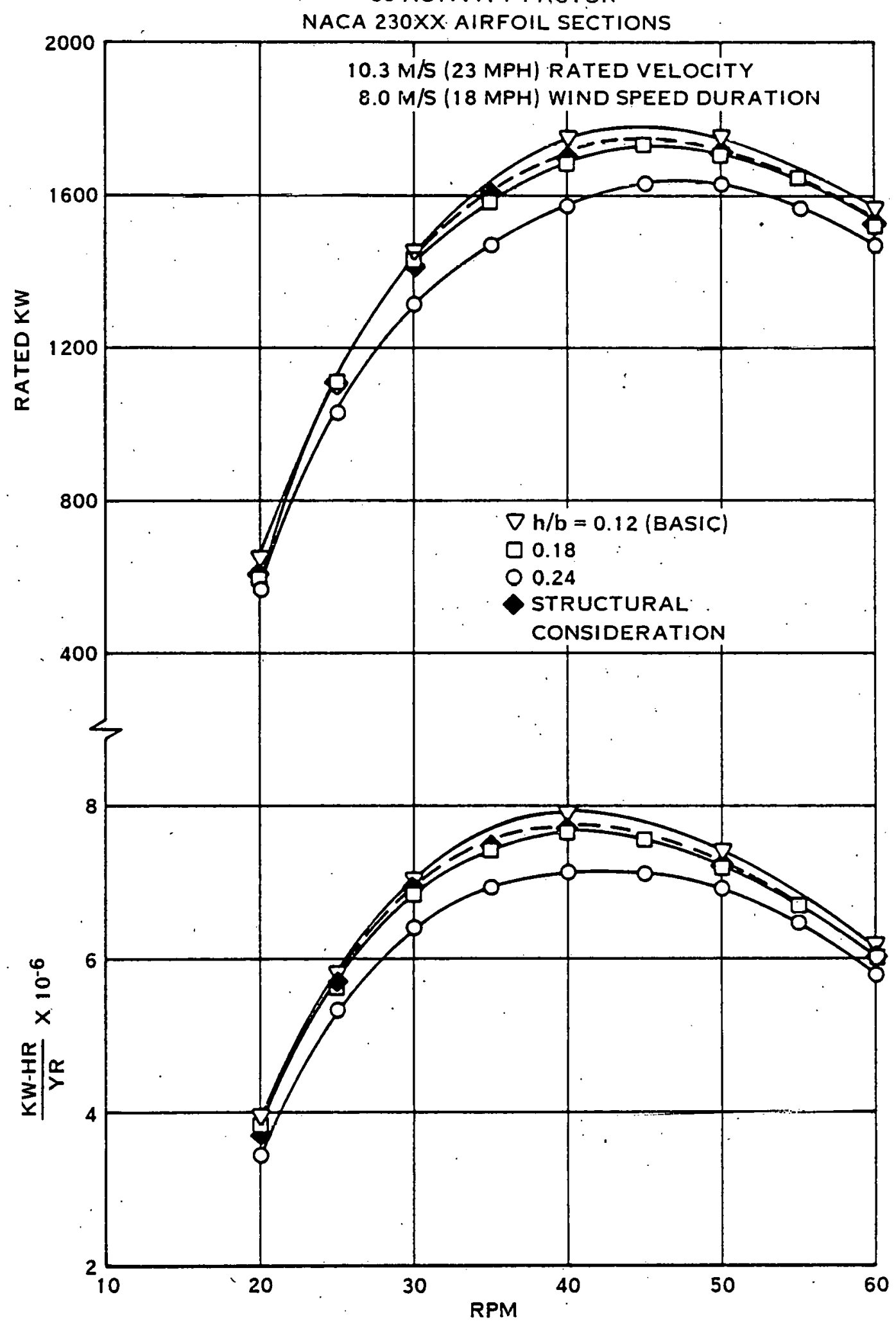

FIGURE 4-21. EFFECT OF THICKNESS RATIO ON THE ANNUAL ENERGY OUTPUT OF 57.9 M (190-FOOT) DIAMETER,2-BLADED WIND TURBINES 


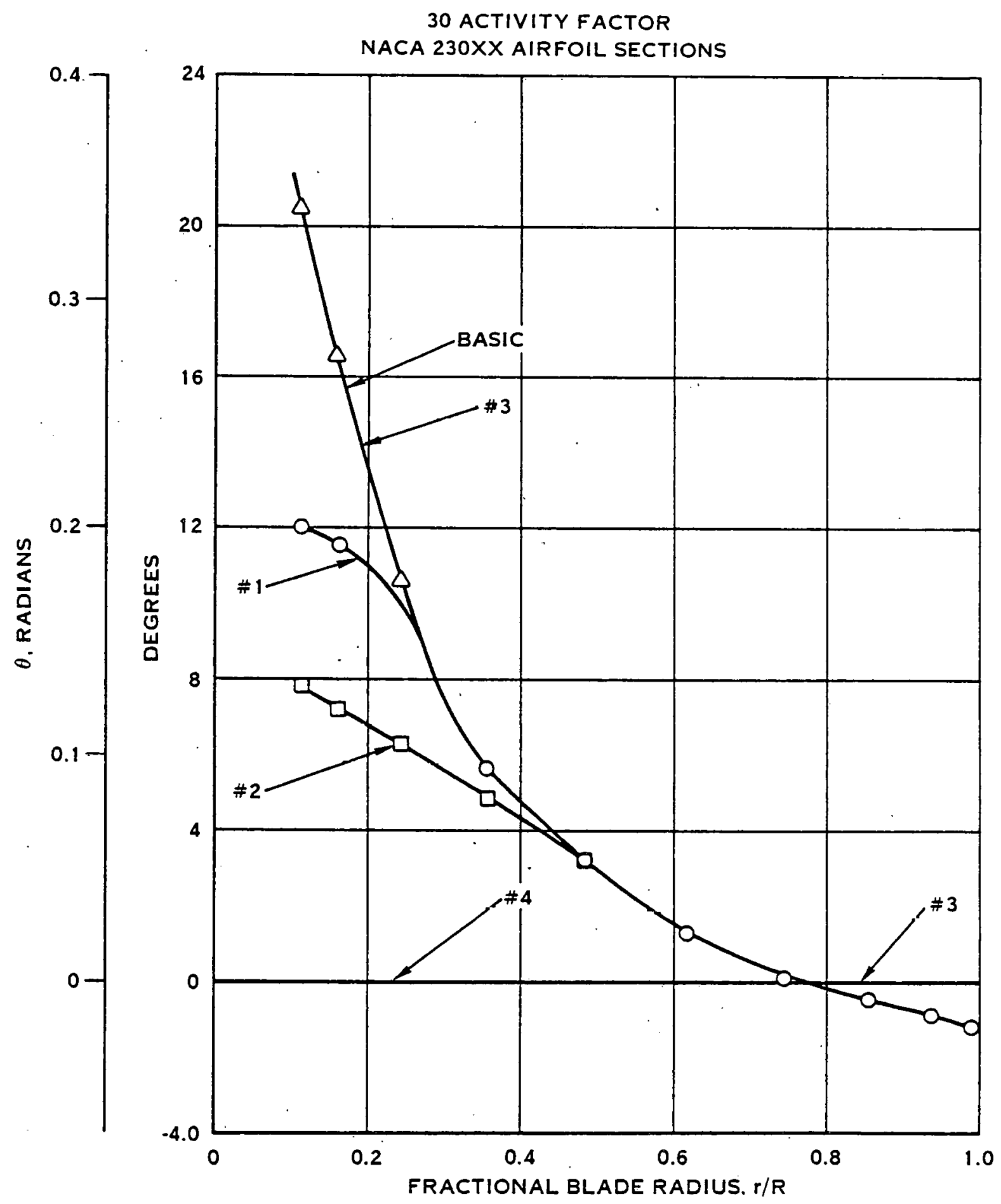

FIGURE 4-22. TWIST VARIATIONS 
30 ACTIVITY FACTOR

NACA 230XX AIRFOIL SECTIONS

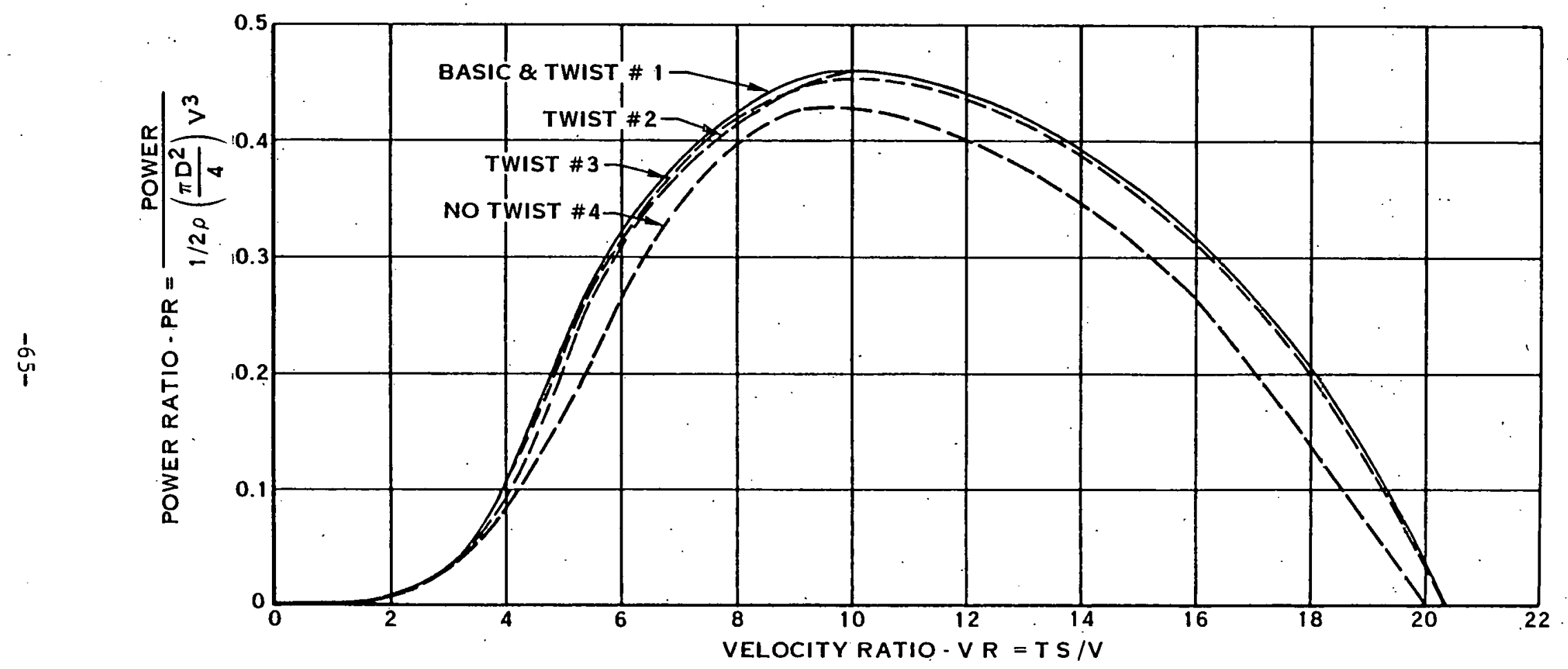

FIGURE 4-23. EFFECT OF TWIST VARIATION 


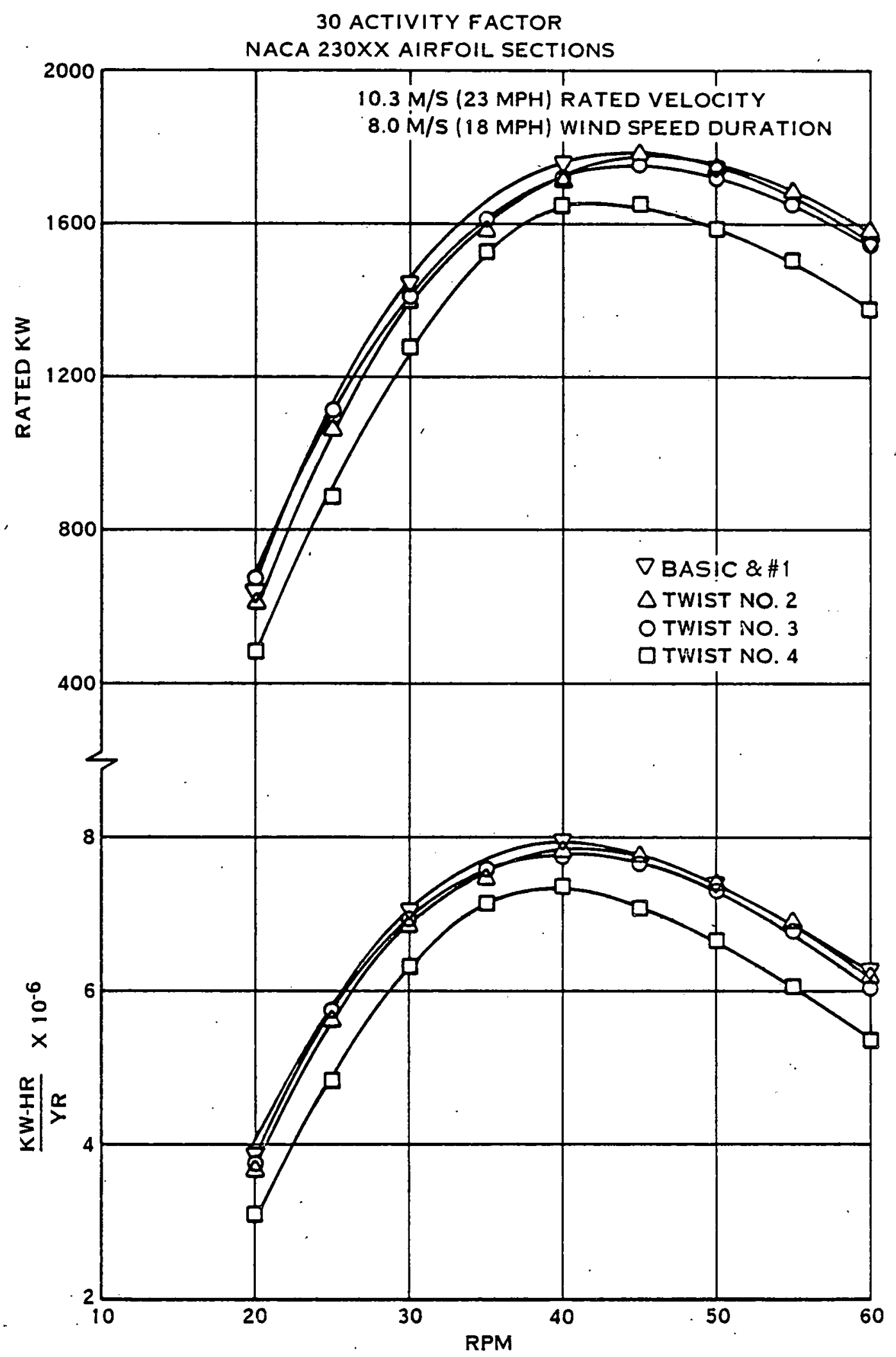

F!GURE 4-24. EFFECT OF TWIST ON ANNUAL ENERGY OUTPUT OF A $57.9 \mathrm{M}$ (190-FOOT) DIAMETER WIND TURBINE 
NACA 23012 AIRFOIL SECTIONS

3 MILLION R.N.

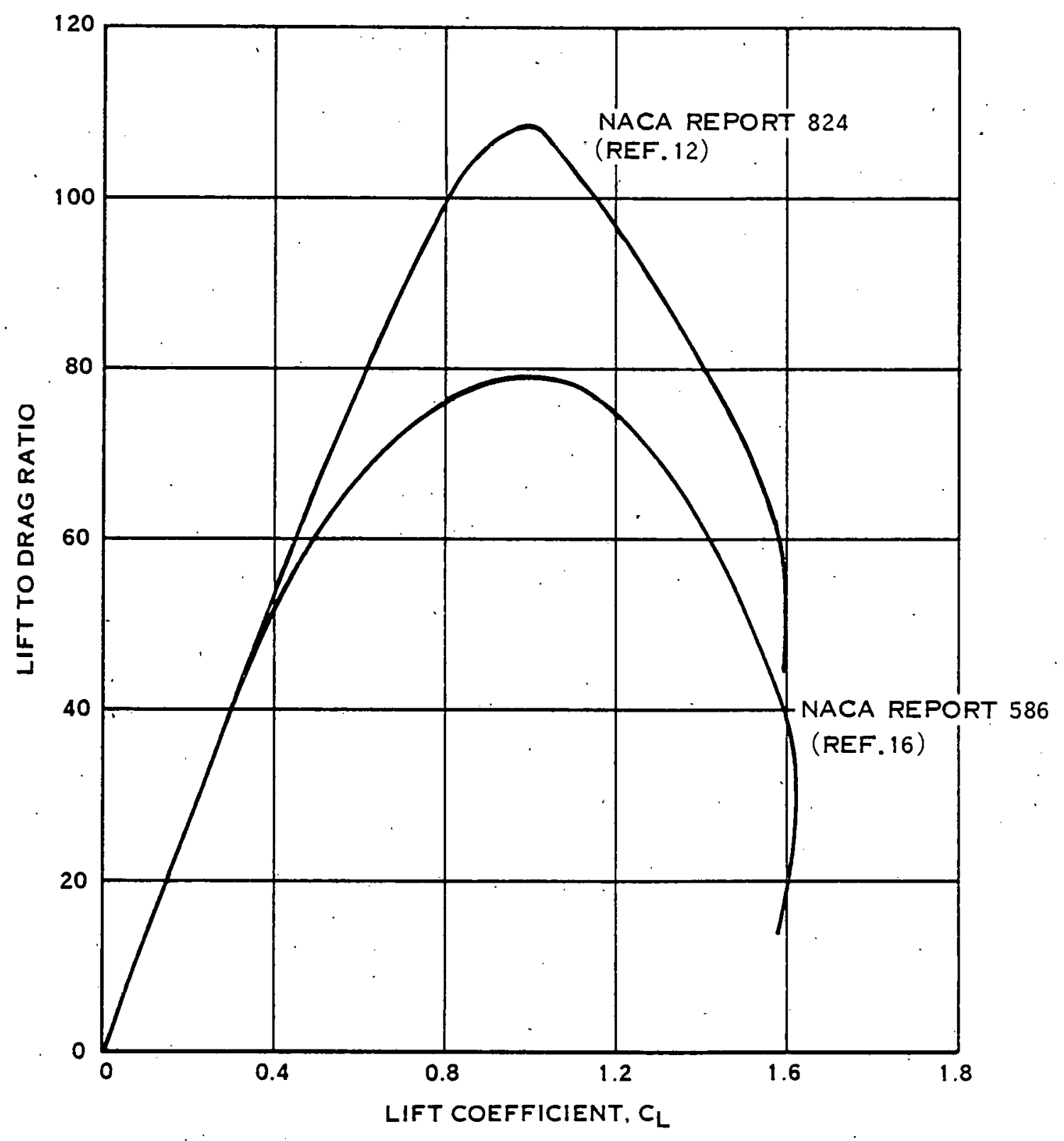

FIGURE 4-25. VARIATION OF LIFT/DRAG RATIO WITH LIFT COEFFICIENT 


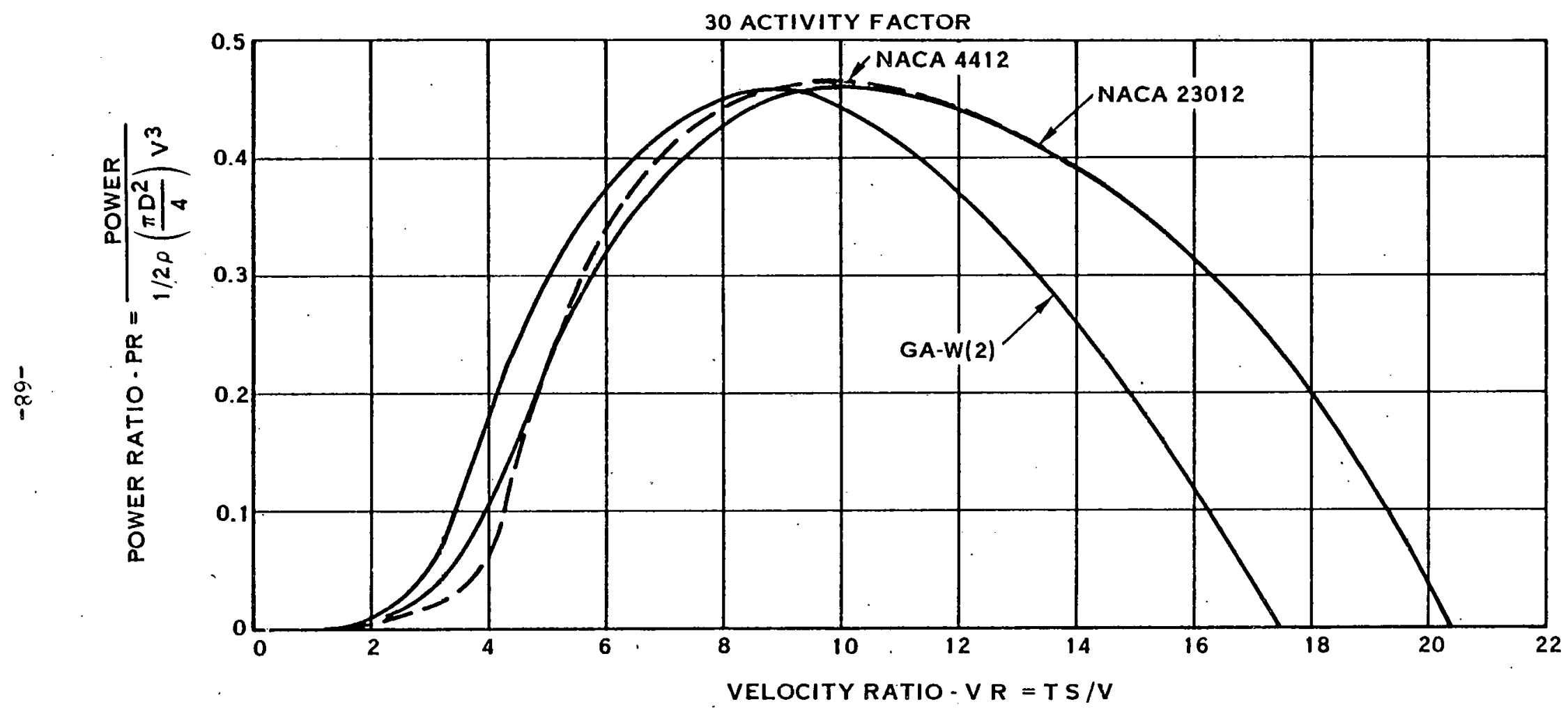

FIGURE 4-26. EFFECT OF AIRFOIL SECTION 


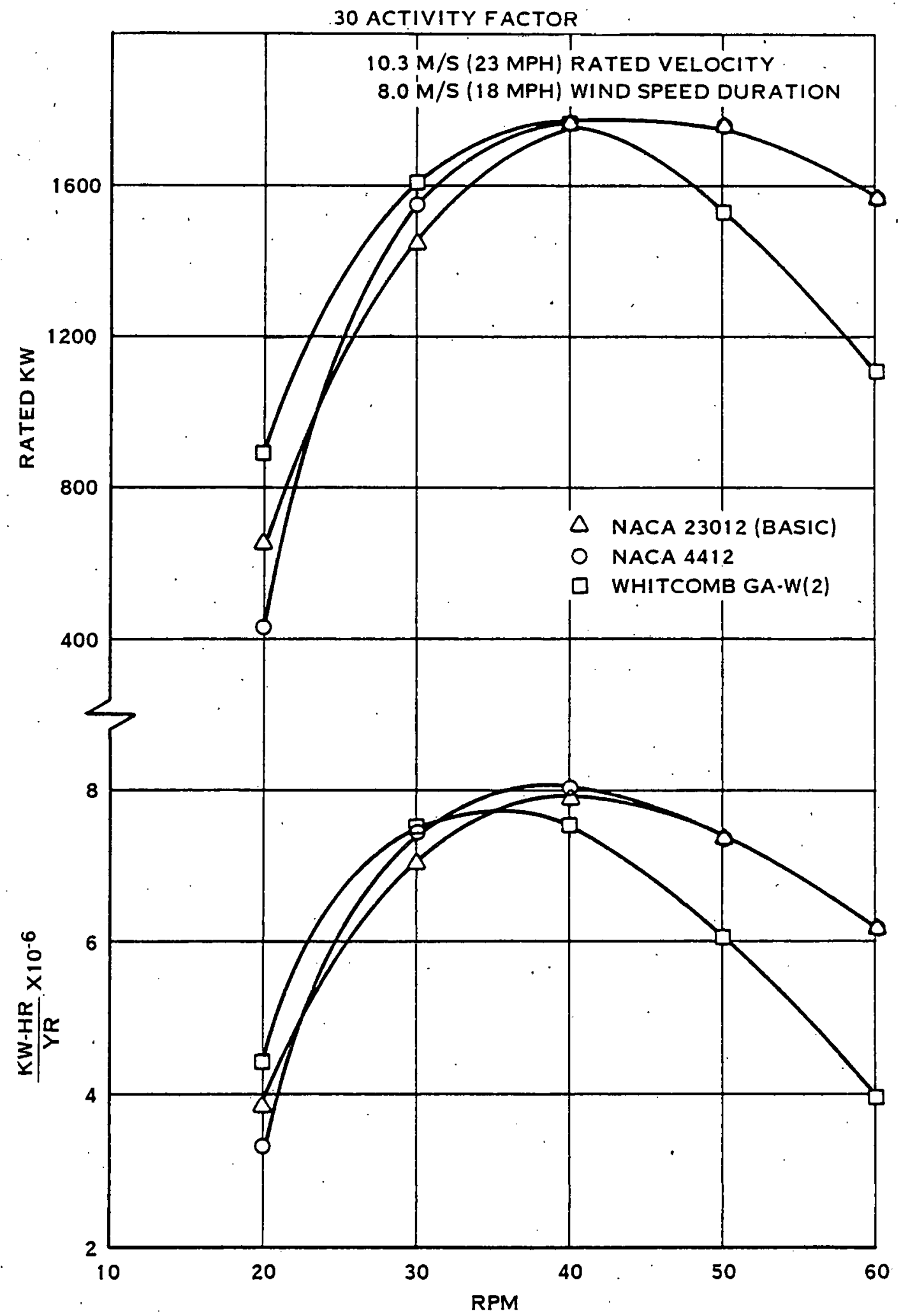

FIGURE 4-27. EFFECT OF AIRFOIL SECTION ON ANNUAL ENERGY OUTPUT FOR A $57.9 \mathrm{M}$ (190 FOOT) DIAMETER WIND TURBINE 
30 ACTIVITY FACTOR

NACA $230 \times X$ AIRFOIL SECTIONS

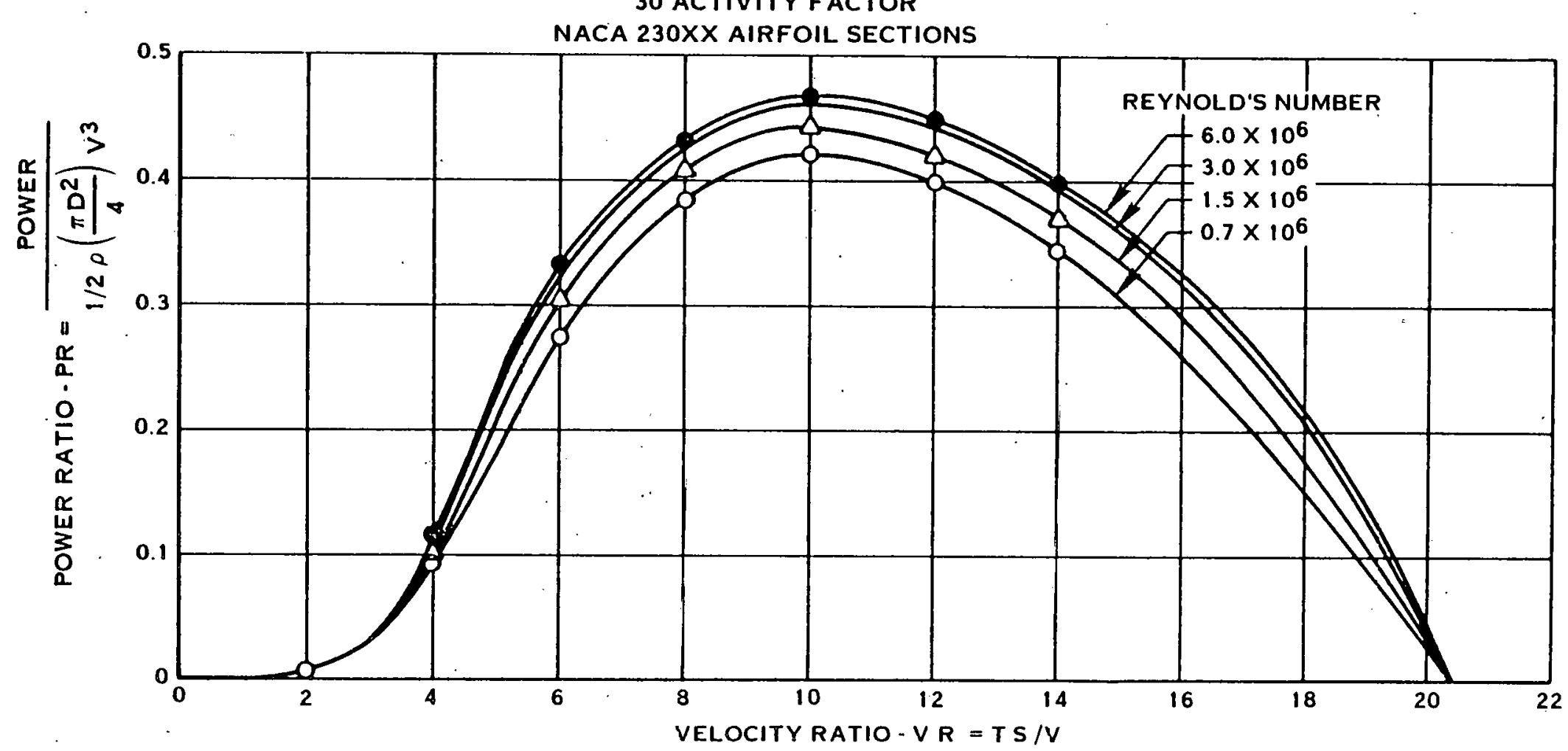

FIGURE 4-28. EFFECT OF REYNOLD'S NUMBER ON THE PERFORMANCE OF THE BASIC WIND TURBINE 

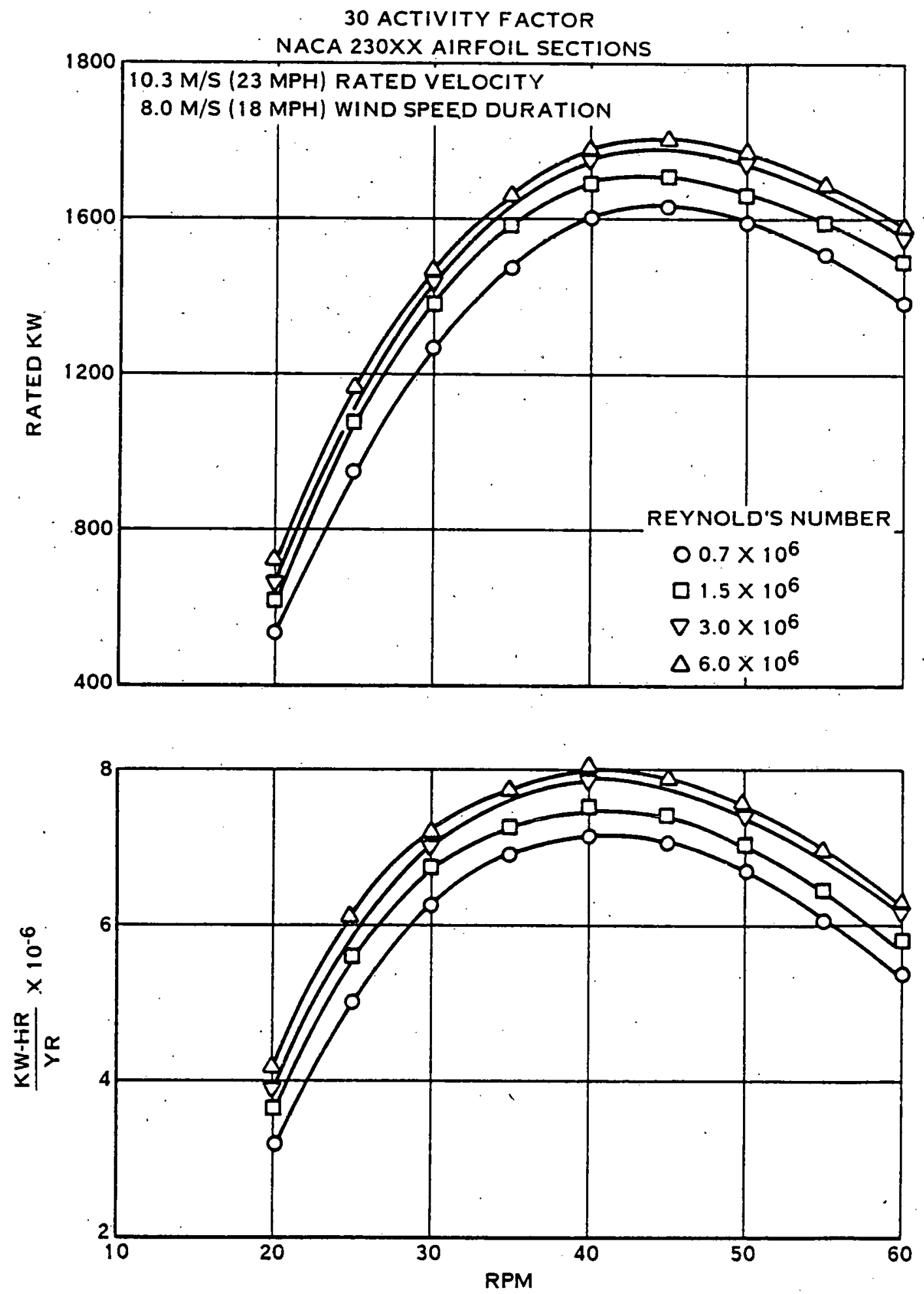

FIGURE 4-29. EFFECT OF REYNOLD'S NUMBER ON ANNUAL ENERGY OUTPUT OF A $57.9 \mathrm{M}$ (190 FOOT) DIAMETER WIND TURBINE 


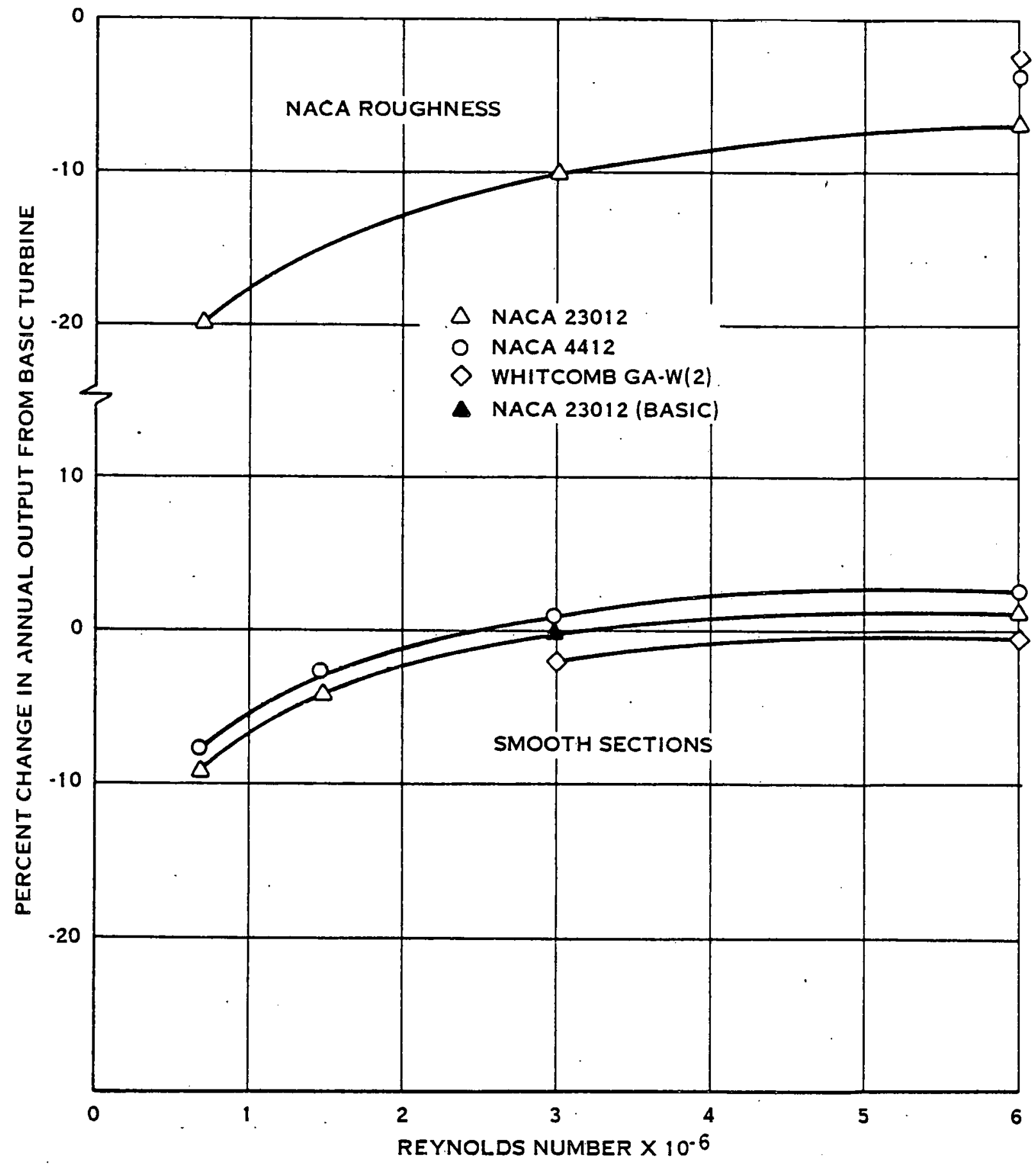

FIGURE 4-30. EFFECT OF REYNOLDS NUMBER AND ROUGHNESS ON ANNUAL ENERGY OUTPUT OF A $57.9 \mathrm{M}$ (190 FOOT) DIAMETER WIND TURBINE 


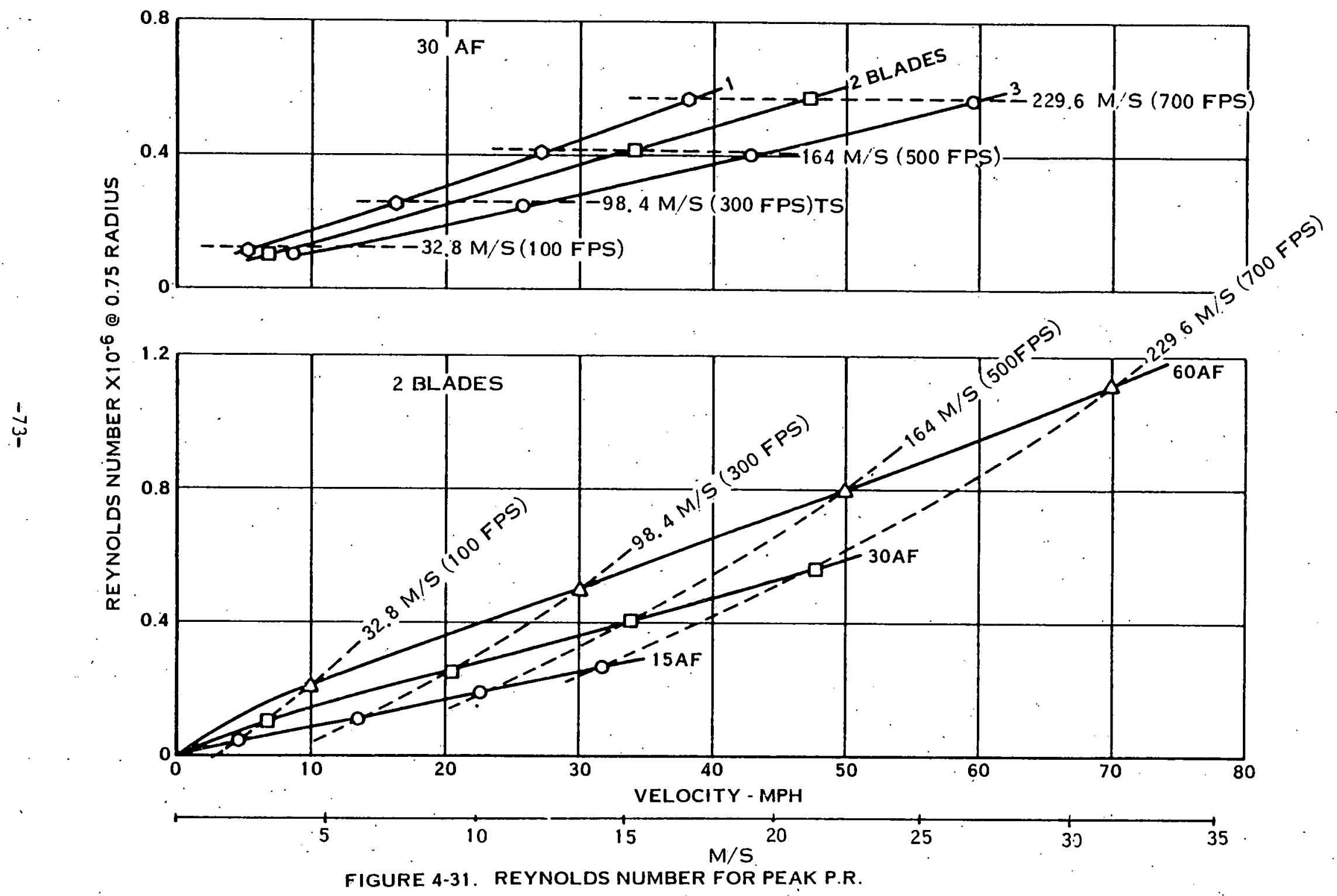

2. $44 \mathrm{M}(8$ FOOT) DIAMETER TURBINE 


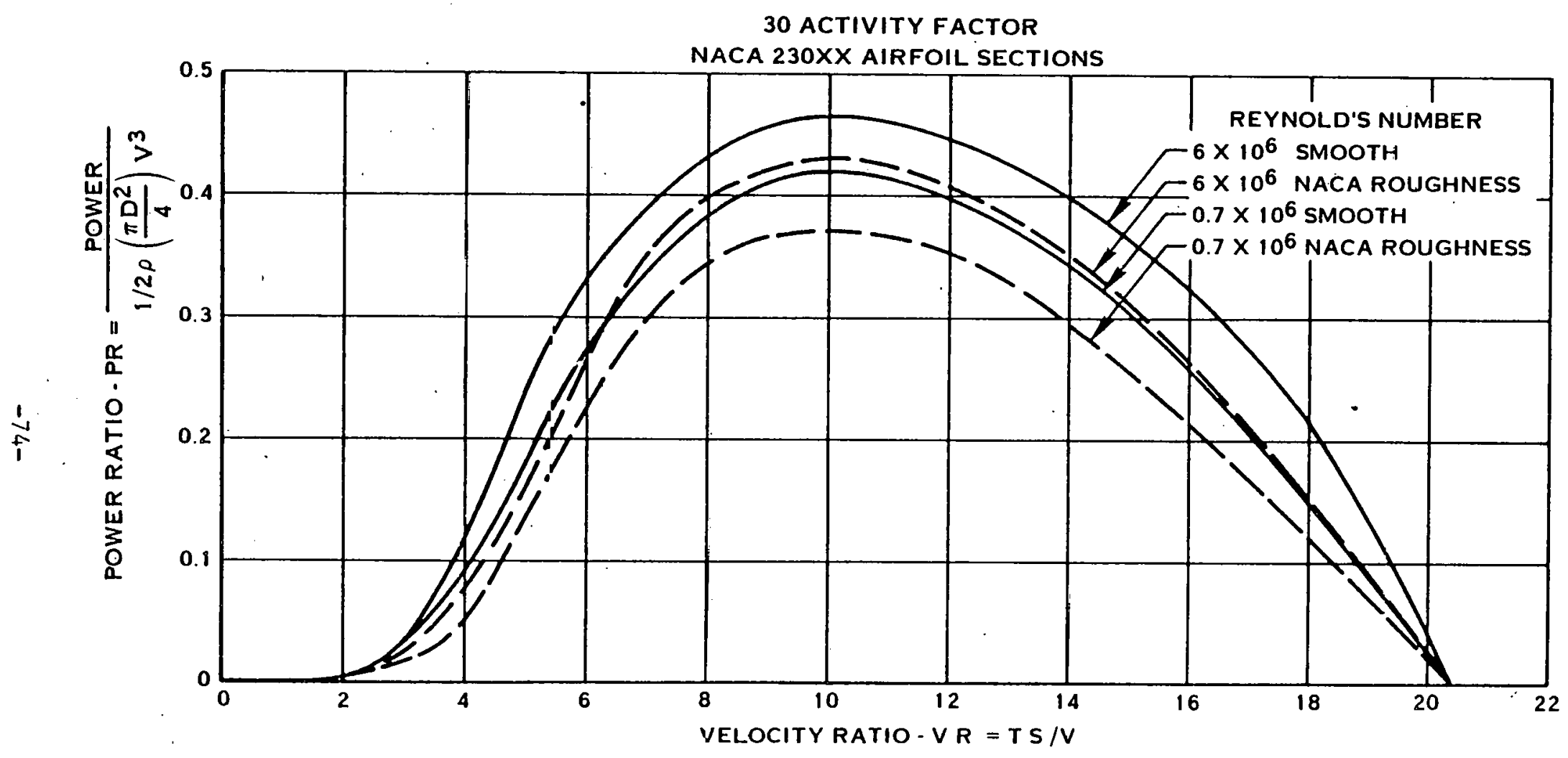

FIGL:RE 4-32. ROUGHNESS EFFECT ON POWER RATIO 

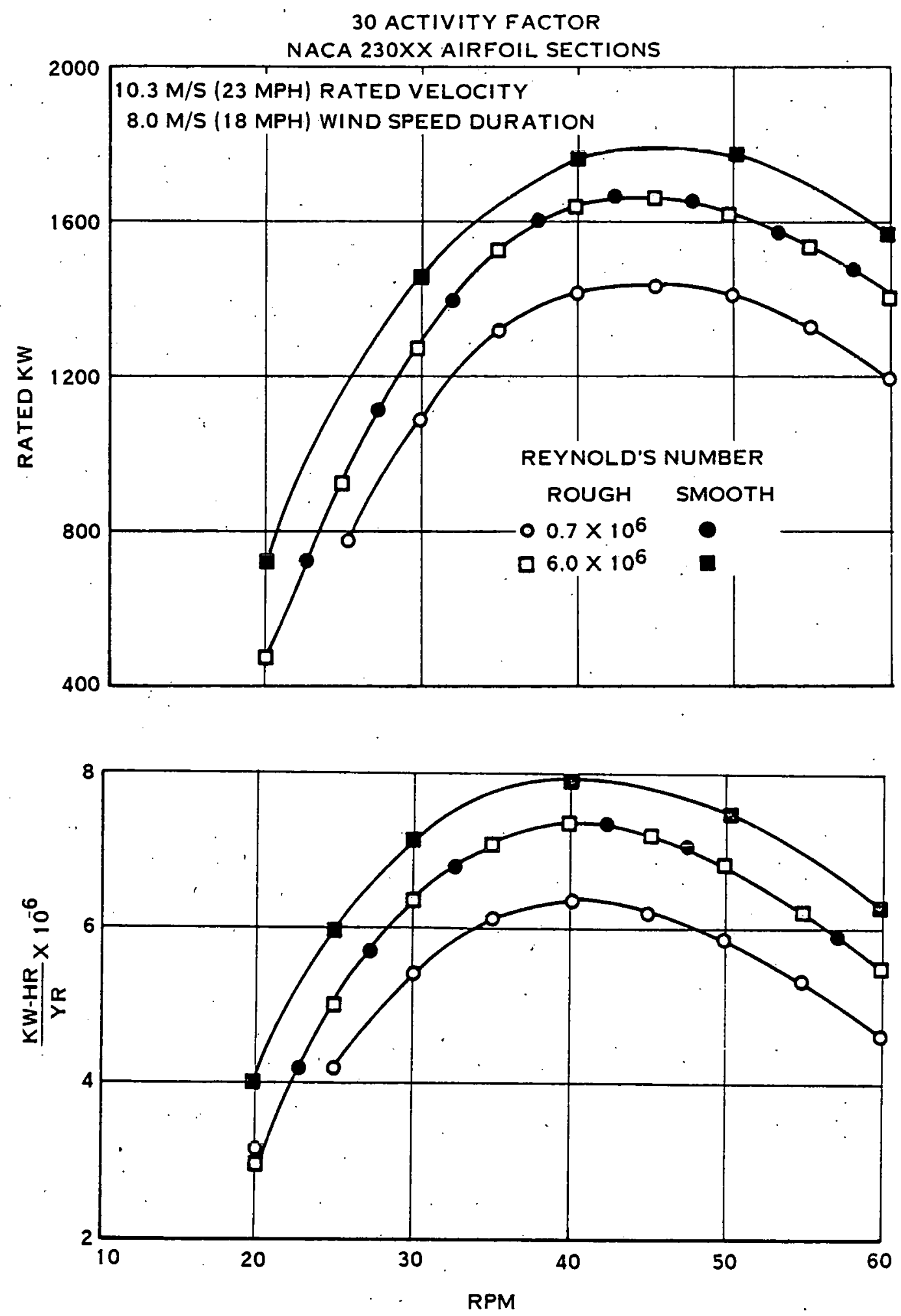

FIGURE 4-33. EFFECT OF ROUGHNESS ON ANNUAL ENERGY OUTPUT OF A 57.9 M (190 FOOT) DIAMETER WIND TURBINE 


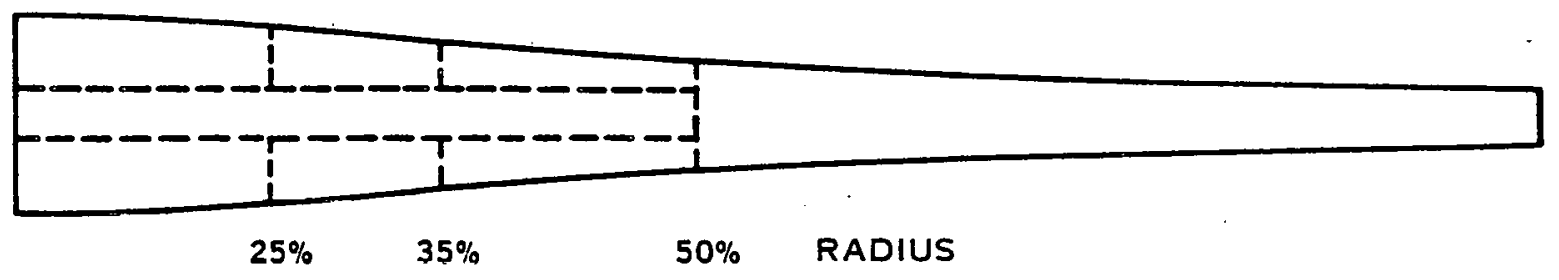

FIGURE 4-34. BLADE ROOT CUT-OFF 


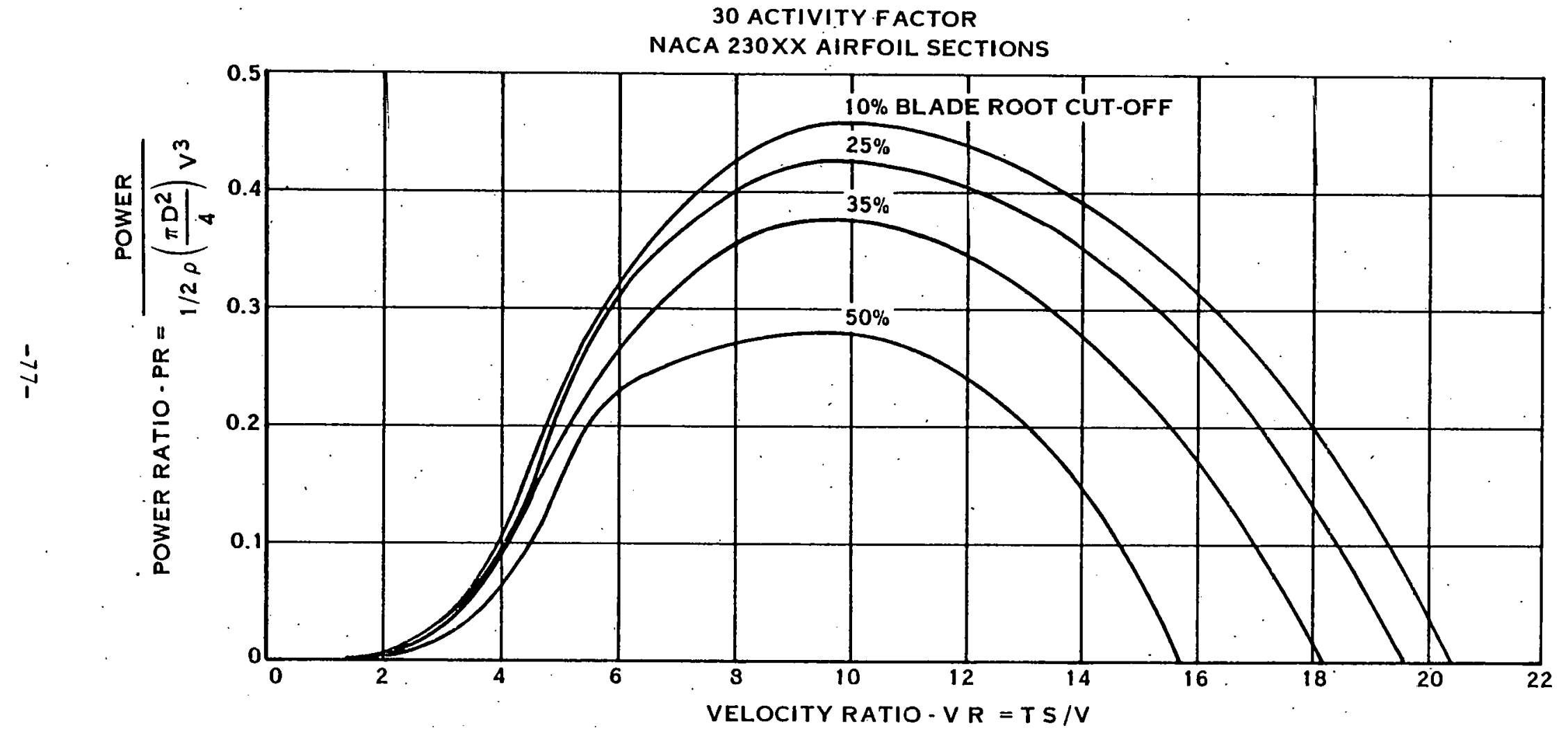

FIGURE 4-35. EFFECT OF BLADE ROOT CUT-OFF 


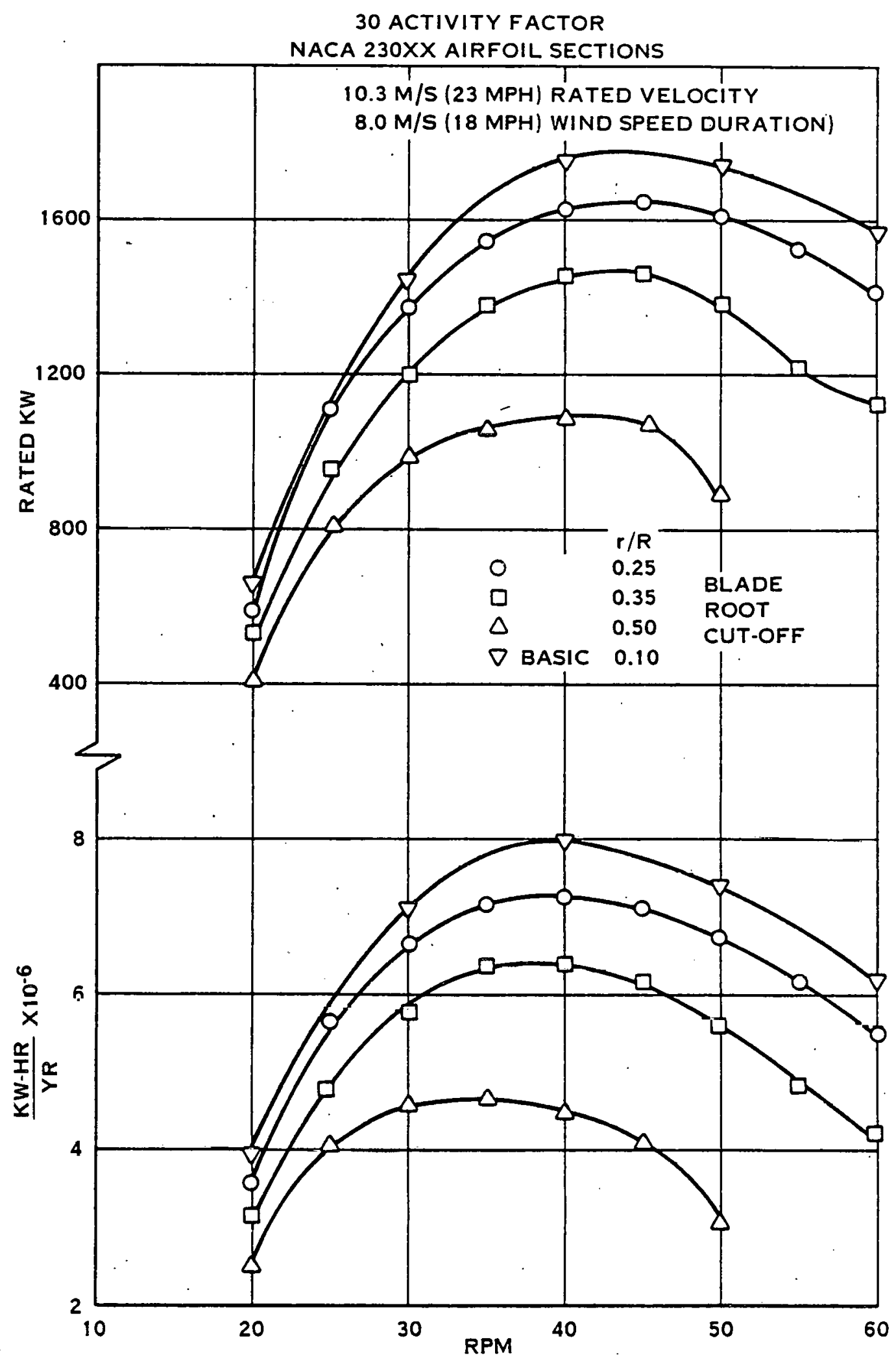

FIGURE 4-36. EFFECT OF BLADE ROOT CUT-OFF ON ANNUAL ENERGY OUTPUT OF A 57.9 M (190 FOOT) DIAMETER WIND TURBINE 


\title{
SECTION 5
}

\author{
LITERATURE SEARCH FOR EXPERIMENTAL DATA
}

In support of the analytical and experimental effort to establish the prime aerodynamic and geometric parameters for wind turbines and to supplement the experimental data obtained from the wind tunnel testing, a continuing review of available 11terature was undertaken. The focus of this effort was to gather pertinent experimental data on full scale and model wind turbines. These data include aerodynamic performance, general operating characteristics and any special observations which could supplement the parametric study and later, the experimental data obtained on the limited number of test configurations included in this program.

The most useful data for this purpose is that obtained under controlled, laboratory conditions covering systematic variations of the aerodynamic and geometric wind turbine parameters and accurately measured performance data on full scale, operating WECS in a natural wind environment. To this end, the available bibliographics from industry, ERDA and other government agencies on wind energy have been searched for such experimental data. Although these bibliographics includes hundreds of references on many categories pertaining to wind energy, only a relatively few contain fully documented experimental data including a description of the test configurations, the test conditions, instrumentation and a complete listing of the test data. Even fewer reports involve systematic testing of the prime variables. Moreover, in some cases, the data are presented without a description of the blade shape characteristics. Thus it is apparent that very little wind turbine performance data of the kind obtained in this test program are available in the literature. Those reports which contain some experimental data are listed in the following bibliography. In addition to these references, some unpublished data have been obtained for use in this program.

Of particular interest in connection with this test program are the data published in items 10 and 15 listed in the bibliography at the end of this section. Moreover they are listed as references 20 and 21 , respectively. In these reports the geometric characteristics of the model tested turbines as well as their performance are presented. Accordingly, these references have been included along with the corresponding data from the models tested in this program and are discussed in Section 11. 


\section{BIBLIOGRAPHY}

1. Culver, F. S., "Performance of Two Successful Windmill Generating Plants," Electrical World, Vol.69, pg. 367-9, February 24, 1917.

2. Blazdell, C., "A Six-Foot Experimental Wind Motor," Model Engineer and Electrician, Vol. 41, pg. 145-51, August 14; pg. 171-8, August 21, 1919.

3. Pancratz, F. J., "Wind Power for Farm Electric Plants," Mechanical Engineering, Vol. 46, pg. 675-82, November 1924.

4. Amans, -; "Sur I'aerodynameque des moulins a vent," Comptes Rendus, Vol. 182, pg. 841-5, March 29, 1926.

5. Prytz, K., "Statens redskabsudvalgs arbejdspr $\phi$ ve med vindm $\phi 11$ er og med enkelte vandloftningsredakaber I aarene 1921-24," Tekniske Forenings Tidsskrift, Vol。50, pg. 113-45, April 1926.

6. Baumhauer, A. G., "Onderzoek von molen-modellen," Ingenieur, Vo1. 48, pg. 160-4, September 1, 1933.

7. Havinga, A., "Proefnemingen met modellen van wind-molens," Ingenieur, Vo1. 49; pg. 17-23, February 23, 1934.

8. Ackeret, J. and Chaille, G., "Untersuchungen am Modell einer Windkraftwerkes," Schweizerische Bauzeitung, Vol. 114, Pg. 41-42, July 22, 1939.

9. Giauert, H., The Analysis of Experimental Results in the Windmill Brake and Vortex Ring States of an Airscrew, Br. R \& M 1026, 1926.

10. N.Y.U., Final Report on the Wind Turbine. Office of Production, Research and Development, War Production Board, PB25370, Washington, -D.C., January $31,1946$.

11. Hütter, U., "Influence of Wind Frequency on Rotational Speed Adjustments of Windmil1 Generators," NASA TT F-15,184, November 1973.

12. Danske, E. F., "Report of the Wind Power Comimittee," NASA TT F-16,062, January 1975.

13. Sweeney, F. E., et al, "Sailwing Windmill Characteristics and Related Topics," Princeton University Report AMS Report No. 1240, NSF/RANN/GI-41891/PR/74/4, Spring 1975.

14. Iwasaki, M., "The Experimental and Theoretical Investigation of Windmills," Research Institute of Applied Mechanics, Kyushu University, Vol. II, No. 8, December 1953.

15. Sulzer, P. Q., Unpublished data on $1.8 \mathrm{~m}(6 \mathrm{ft}$ ) wind turbines. 
SECTION 6

SELECTION OF MODEL WIND TURBINE CONFIGURATIONS

The objectives of this program include the establishment of the performance trends of a series of optimum rotors incorporating variations of the prime geometric parameters, the evaluation of the wind turbine design and performance prediction methods and the assessment of two advance aerodynamic methods.

In consideration of these objectives, the results of the parametric study discussed in Section 4 were carefully analyzed on the basis of establishing the wind turbine models to be tested in the wind tunnel. The blade shape parameters deemed most significant in fulfilling the program objectives and which represent those most requiring experimental confirmation were selected after careful. deliberation. As pointed out in the parametric study discussion, the 2 bladed/30 AF/Trapezoidal Planform/NACA 230XX airfoils is an optimum design and has been designated the reference or basic configuration. Moreover, this design is essentially the same as that established in the study conducted by General Electric and Hamilton Standard on large WECS (ref. 5). Accordingly, this configuration has been selected as the reference model wind turbine.

From a review of Table 4-1 from Section 4 , it is noted that only increasing number of blades and airfoil type resulted in an improvement in annual power output over the reference wind turbine. Large deviations from the reference design in twist distribution, thickness ratio distribution and planform distribution could reduce output power from 7 to 13 percent, while blade root cut-offs greater than 15 percent of the radius rapidly reduce performance. However, design and manufacturing studies do not indicate any significant cost advantage in deviating grossly from the optimum blade shape characteristics with the exception that thickness ratio distribution is usually dictated by structural and cost requirements. Thus, in view of the limited number of models, it has been judged that these latter parameters should not be investigated in this test program.

Accordingly, in addition to the basic model wind turbine selected above, three additional models incorporating the optimum trapezoidal planform with activity factors per blade of 15 and 60 and NACA Series $230 \mathrm{XX}$ airfoil sections and of $60 \mathrm{AF}$ with NACA $44 \mathrm{XX}$ airfoils were selected for wind tunnel testing. With these models many of the prime variables studied in the parametric study can be experimentally investigated. These include number of blades, activity factor, airfoil type, Reynolds number, and blade surface roughness and waviness. Moreover, these models encompass the practical range of design velocity ratio. The blade characteristics for these optimum model wind turbines are shown in figures $6-1,6-2$, and $6-3$ and summarized in the following table.

Model Configurations

\begin{tabular}{cccc} 
MODEL & ACTIVITY FACTOR & NO. BLADES & AIRFOIL SECTION \\
\hline 1 & 15 & 2 & $230 \times \mathrm{XX}$ \\
$2 \star$ & 30 & 2 & $230 \mathrm{XX}$ \\
3 & 30 & 3 & $230 \times \mathrm{XX}$
\end{tabular}




\begin{tabular}{|c|c|c|c|}
\hline MODEL & ACTIVITY FACTOR & NO. BLADES & AIRFOIL SECTION \\
\hline 4 & 30 & 1 & $230 \mathrm{xx}$ \\
\hline 5 & 60 & 2 & $230 x x$ \\
\hline 6 & 60 & 2 & $44 X X$ \\
\hline
\end{tabular}

Each model incorporates the twist distribution required for optimum loading. The thickness ratio distribution for all models is that corresponding to practical structure consistent with cost effective fabrication and materials.

It should be pointed out, that some modification to these blade shape characteristics have been required to provide structurally sound models. These modifications are not expected to compromise the objectives of the program and are discussed later in Section 7.

The curves in figure 6-4 present the peak performance spectrum of a family of optimum wind turbines in terms of power coefficlent versus velocity ratio for a range of turbine blade number. The points noted on the curves indicate the design points for the test models.

As indicated above in Section 4, the reliability of the performance analysis for the one-bladed wind turbine is considered to be questionable. The literature shows a wide variation in the predicted performance level of this wind turbine. Thus, at least one model one-bladed wind turbine has been included in the testing.

The three bladed, model has been included because of the high predicted performance and low sensitivity to tower interaction albeit a less cost effective configuration.

It has been shown that as design velocity ratio is increased, the wind turbine $A F$ must be decreased. This, together with the increasing tendency of the blade wakes to interfere with the flow through the rotating blading as velocity ratio increases makes the selection of the $15 \mathrm{AF}$ model of prime importance in pinning down the maximum practical design velocity ratio.

The well proven NACA $230 \mathrm{XX}$ airfoil series was selected as the basic airfoil for the wind turbine models because of the demonstrated high lift to drag ratios over a wide range of lift coefficients at $\mathrm{RN}$ appropriate to large WECS. It is recognized that the forward loading of this airfoil series makes it more sensitive to surface roughness than one with more uniformly distributed chordwise loading. Thus the NACA $44 \mathrm{XX}$ airfoil series and the new Whitcomb, GA(W)-2 airfoils were investigated as reported in the parameteric study. In view of the available experimental data on the NACA 44XX series at low RN and its attractive performance characteristics, this airfoil was included as a test variable. The $60 \mathrm{AF}$ model will be tested with both NACA 230XX and NACA 44XX airfoil sections since this model will permit the comparison of airfoil types to be made at the highest test RN.

Since wind tunnel testing of practical wind turbine model sizes cannot attain full scale $\mathrm{RN}$, it is important that the largest diameter models be tested to minimize RN effects (fig. 4-30). Therefore, for this test program, a $2.44 \mathrm{~m}$ ( $8 \mathrm{ft.}$ ) diameter was chosen for the models; the largest 
diameter compatible with the wind tunnel test section and existing test hardware. In this connection, figure 6-6 shows the variation of RN with tip speed, $A F$, number of blades and wind velocity. Inasmuch as increased $A F$ provides the highest value of $\mathrm{RN}$ for a given operating condition, the $60 \mathrm{AF}$ models were considered important configurations for the test program.

Both blade surface roughness and waviness were included on the 60 AF model in consideration of manufacturing tolerances due to cost effective blade fabrication processes and possible erosion under severe environmental conditions which could have a deleterious effect on performance.

Thus, the experimental data produced on these four different blade designs (providing six or seven test configurations) will establish a comparison with predicted performance of each test configuration and fulfill the program objectives stated above.

Because of the large number of variables in wind turbine geometry as discussed in Section. 4 and other installation variables not considered in this program, the selection of the four blade designs described herein required considerable deliberation to best assure that the objectives of the program would be accomplished. Thus it may be appropriate to investigate several other of these parameters as additional models to this program or in later programs. 


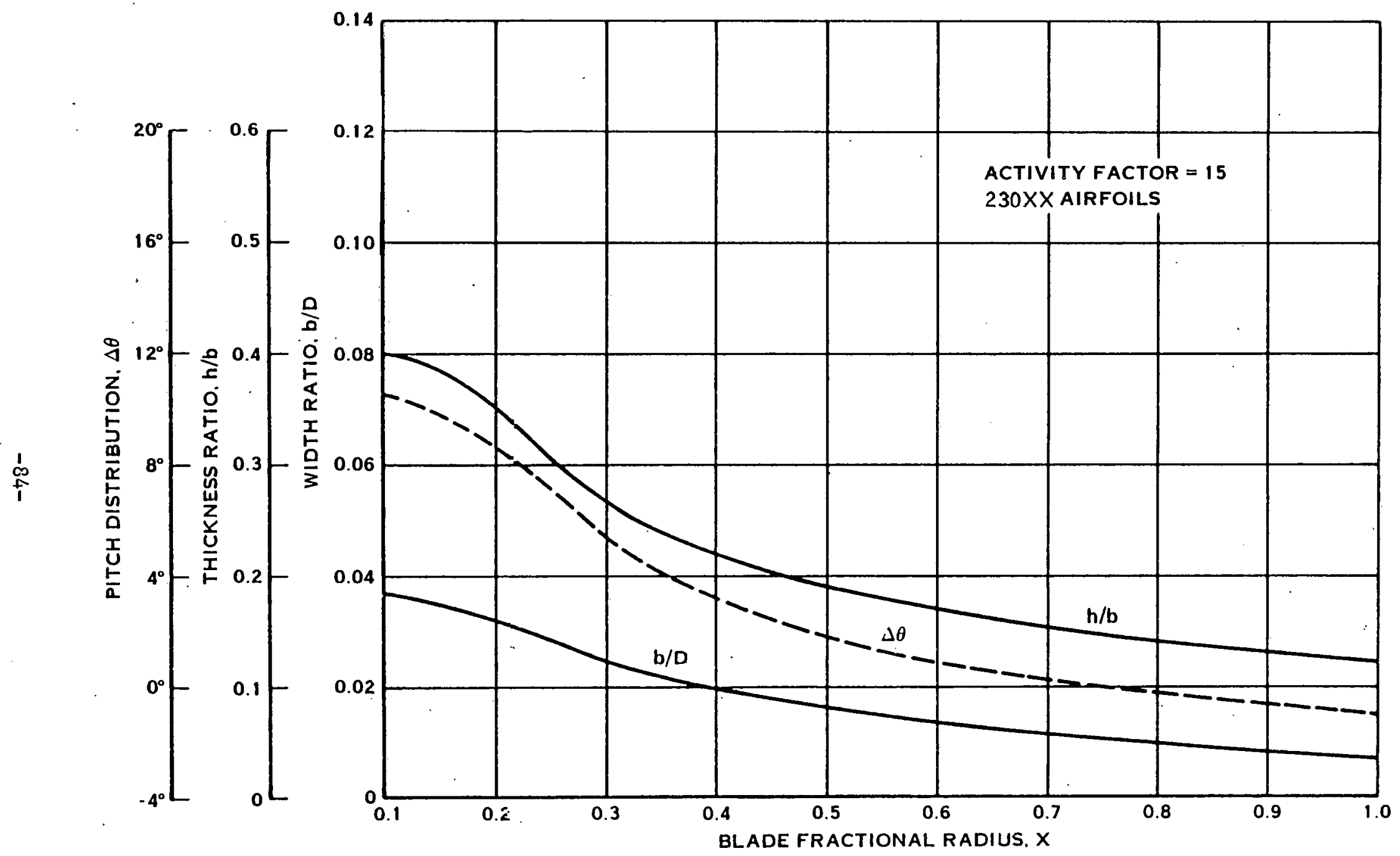

FIGURE 6-1。 MODEL 1 WIND TURBINE BLADE CHARACTERISTICS 


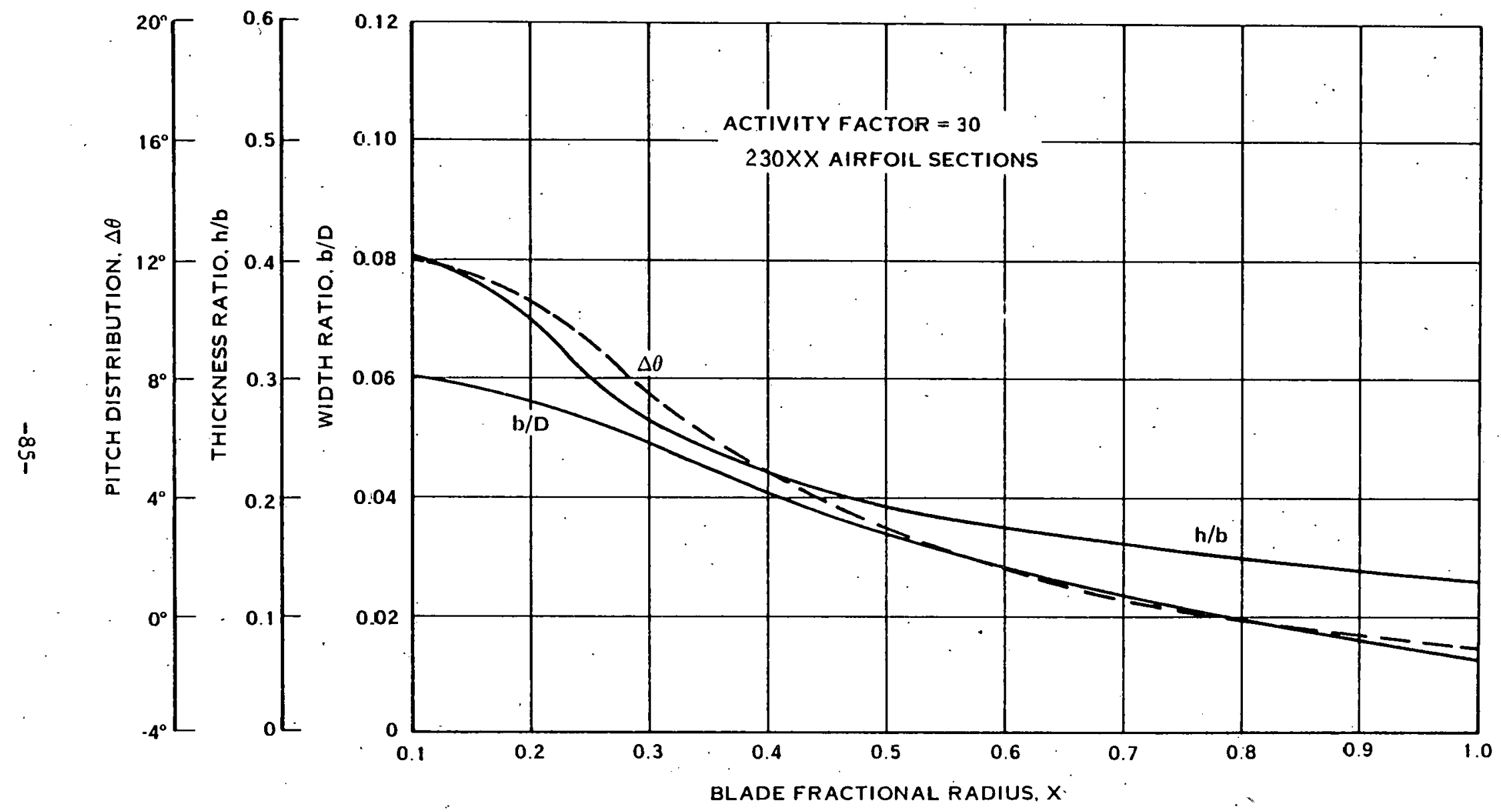

FIGURE 6-2. MODFLS 2, $3 \& 4$ WIND TURBINE BLADE CHARACTERISTICS 


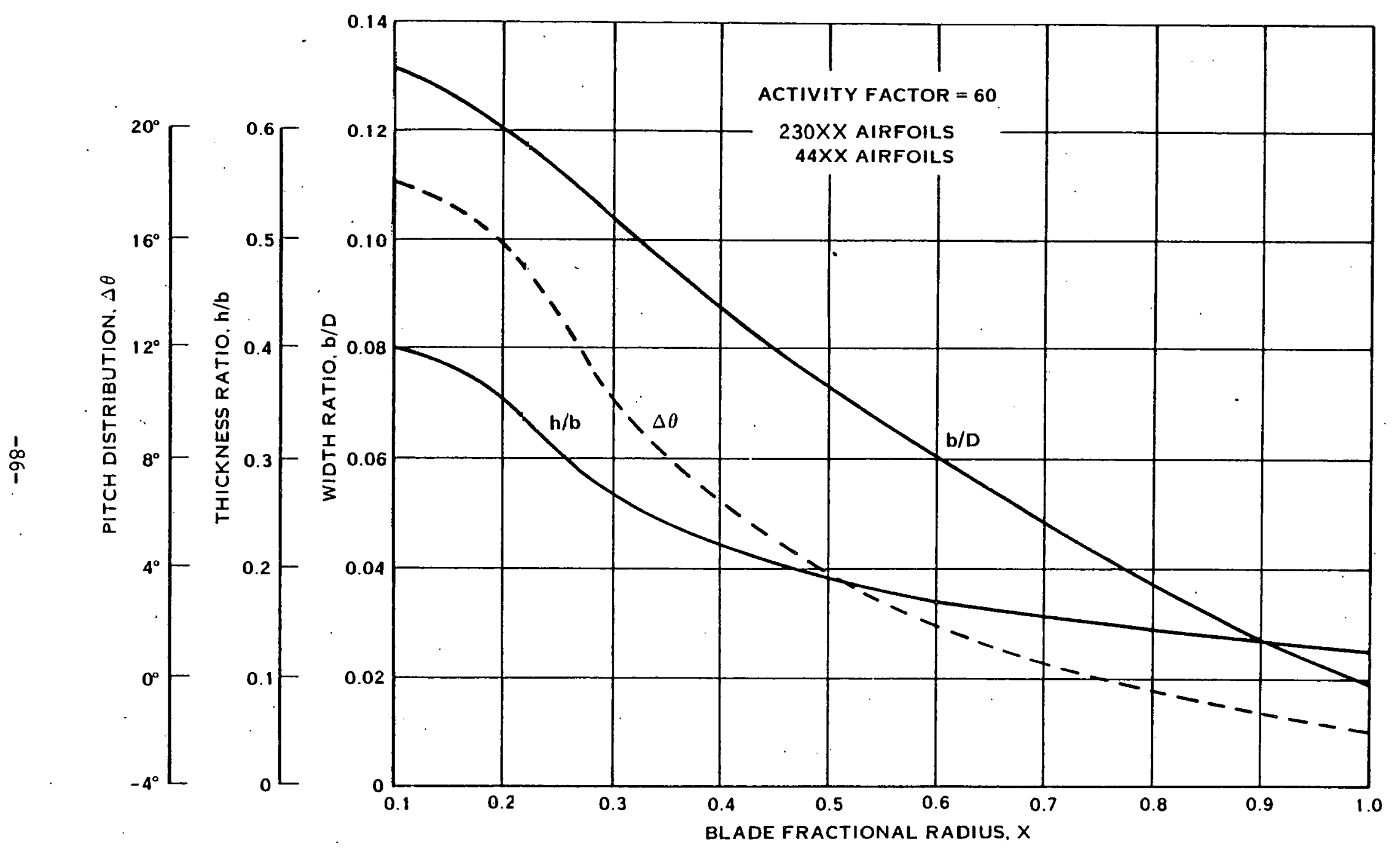

FIGURE $\dot{0}-3$. MODELS $5 \& 6$ WIND TURBINE BLADE CHARACTERISTICS 


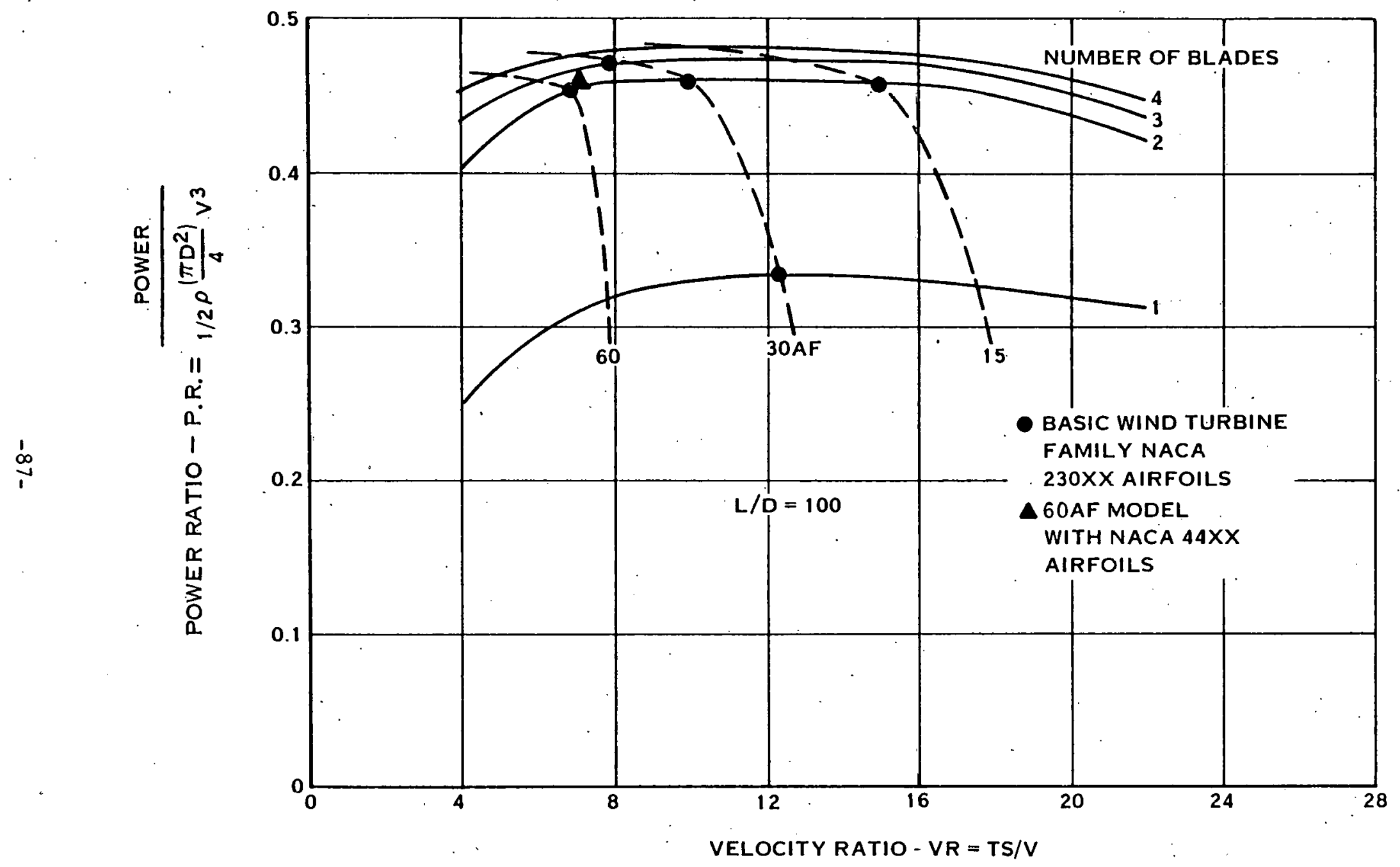

FIOURE 6-4. WIND TURBINE ROTOR PERFORNANCE FOR OPTIMUM BLADE DESIGNS 


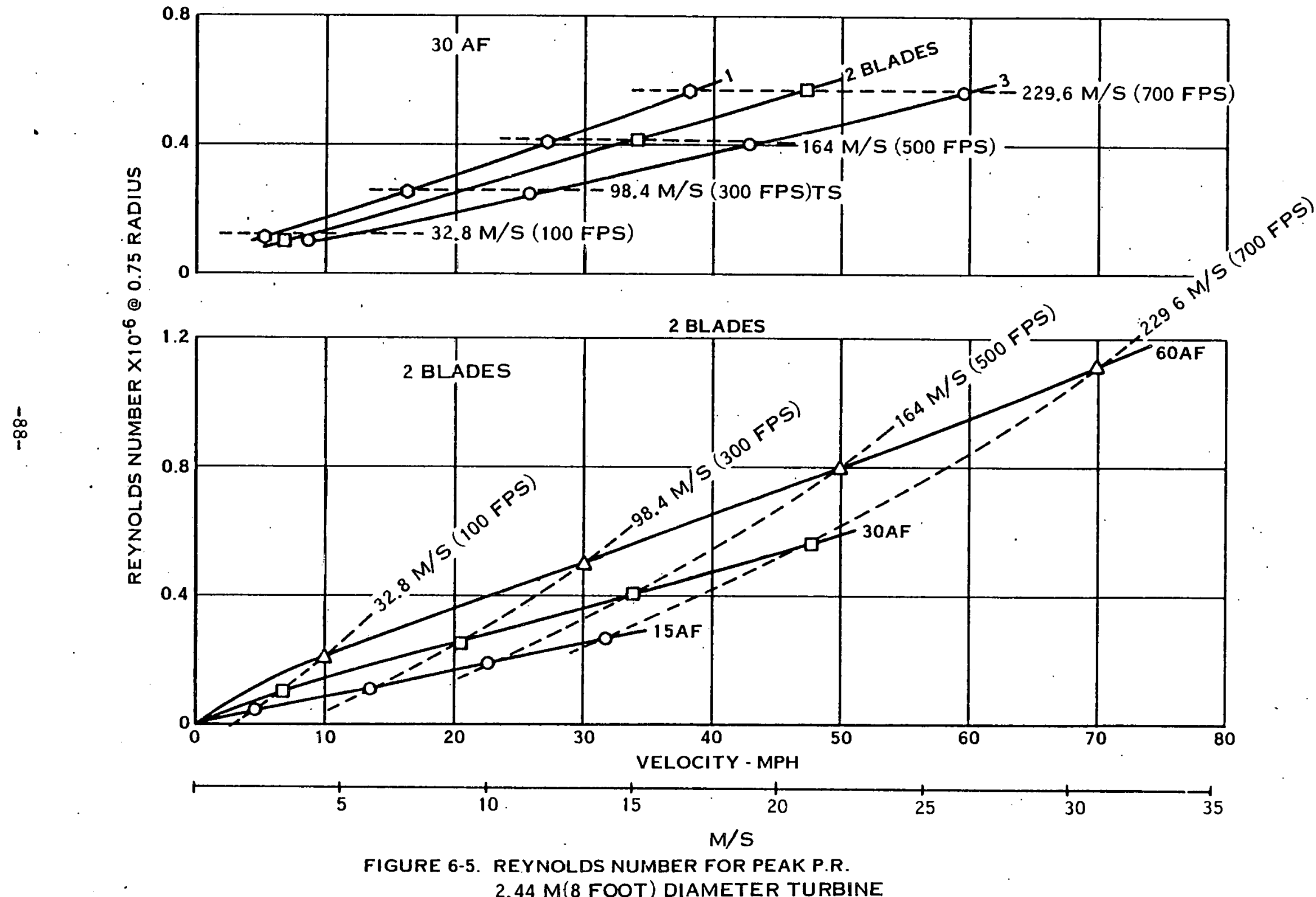




\section{SECTION 7}

\section{DESIGN OF MODELS}

\subsection{PHILOSOPHY}

The design of the model blades has been exercised so as to achieve blade configurations which provide as closely as possible the aerodynamic characteristics desired. Compromises which allow for safe use, increased operating envelopes, and economical manufacture have affected the final configurations.

Initial studies at Hamilton Standard of wind turbine blading dealt with the 30.5 meter $(100 \mathrm{ft})$ diameter size. The NASA/G.E./H.S. (ref. 5) study program expanded these studies up to 61 meter $(200 \mathrm{ft})$ size. These study blades included hollow construction of monocoque, semi-monocoque, and spar/shell concepts. It was from this study work that the model blade shapes were derived.

\subsection{ACTIVITY FACTOR BLADE}

When work was initiated on the model configurations, the $30 \mathrm{AF}$ blade utilizing the thickness and planform characteristics developed in the NASA/G.E./H.S. study was selected as the first model blade.

This blade was scaled down from 59.1 meters ( $194 \mathrm{ft}$ ) to 2.44 meters ( $8 \mathrm{ft}$ ) diameter, changed from hollow construction to solid aluminum and permitted to operate at tip speeds up to $213 \mathrm{~m} / \mathrm{s}(700 \mathrm{ft} / \mathrm{sec})$ in order to achieve relevant Reynolds numbers. The aluminum model blade had ample strengths for operation at a wind velocity of $35.8 \mathrm{~m} / \mathrm{s}(80 \mathrm{MPH}), 213 \mathrm{~m} / \mathrm{s}(700 \mathrm{ft} / \mathrm{sec})$ tip speed and with inflow distortion up to $45^{\circ}$ yaw. Figure $7-1$ shows the stress relative to material allowables. for this operating condition at the maximum stress point in the airfoil.

Modifications to the blade were necessary to place bending and torsional frequencies properly. Because the model blade is of solid construction, frequency modification can only be accomplished by chord and thickness changes. This contrasts to the full scale blade construction using a hollow structural spar and thin airfoil shell where spar and shell wall thickness can be varied as an additional strong parameter influencing blade stiffness. In order to maintain AF and blade planform shape, thickness was chosen rather than chord as the parameter to iterate in obtaining proper frequency placements. Composite reinforcement could have been used but this approach was judged too costly.

Figure 7-2 indicates the extent of thickness modifications necessary in the placement of blade frequencies. Figure 7-3 shows the final frequency placements for the $30 \mathrm{AF}$ blade. The two-per-revolution excited first bending mode is placed 10 percent above the maximum operating speed. The three-per-revolution excited first mode bending limits operation over a small band in the lower speed range. It will probably only be excited in the three-bladed version. Higher order excitations and more complex mode shaped responses are basically out of the testing envelope. The four-per-revolution second mode is in the corner of the envelope but judged as too weak an excitation to cause operating limitations. Blade rocking may be experienced below 400 RPM. 


\subsection{ACTIVITY FACTOR BLADE}

The $15 \mathrm{AF}$ model blade was the second blade design. The first configuration investigated utilized thickness distribution identical to the final configuration of the $30 \mathrm{AF}$ model blade. A first approximation of frequency placement can be established by scaling from the $30 \mathrm{AF}$ blade. Here blade length 1.22 meters ( $4 \mathrm{ft}$ ) will be identical for each blade. Chord will be approximately $1 / 2$ due to the $30 \mathrm{AF}$ to $15 \mathrm{AF}$ requirement and thickness $1 / 2$ for the same $t / b$ distribution.

Blade bending frequency $=\mathrm{f}\left[\frac{\mathrm{k}}{\mathrm{m}}\right]^{1 / 2}$

$$
\begin{aligned}
& =\mathrm{f}\left[\frac{\text { chord (thickness) }}{\text { (chord)(thickness) }}\right]^{1 / 2} \\
& =\mathrm{f}[\text { thickness }]
\end{aligned}
$$

From this it is readily apparent that the $15 \mathrm{AF}$ blade must operate above its first bending critical if it is to be of solid aluminum construction and have no composite reinforcement. Super-critical operation of model and full scale blades has been accomplished successfully with Hamilton Standard propellers on several occasions in the past and was judged quite acceptable for this use. Figure $7-4$ shows the final critical speed placements.

The next consideration is that of stresses. Here a simplified approximation of scaling stresses from the $30 \mathrm{AF}$ blade of the same length indicates unacceptable results.

$$
\begin{aligned}
\text { stress } & =f\left[\frac{\text { bending moment }}{\text { section modulus }}\right] \\
& =\mathrm{f}\left[\frac{(\text { chord })}{\text { (chord)(thickness) } 2}\right] \\
& =\mathfrak{f}\left[\frac{1}{\text { thickness }} 2\right]
\end{aligned}
$$

Since the desired thickness of the $15 \mathrm{AF}$ blade is $1 / 2$ that of the $30 \mathrm{AF}$ blade, stress could be expected to be four times that of the $30 \mathrm{AF}$ blade. This stress condition would be completely unacceptable. An important factor. relative to blade air load bending capacity not accounted for in the above simplification is the negating effect of centrifugal restoring moment.

As the air load flexes the blade down wind, this displacement produces an offset of the mass of the blade. This mass under the rotational centrifugal effect tends to straighten the blade radially. In this manner portions of the air loads are supported by centrifugal load rather than bending in the blade itself.

The effectiveness of the centrifugal restoration was evident when the $15 \mathrm{AF}$ blade was run in the detail design programs. Steady blade stressing did not increase over that of the $30 \mathrm{AF}$ blade design. This result initiated additional study of the sensitivity of these blades to centrifugal restoring loads. 
Theoretical blades of zero mass were programmed. From these calculations it was found that at the maximum stress blade stations, $3 / 4$ of the air load bending moment was centrifugally supported on the $30 \mathrm{AF}$ blade and on the 15 AF blade $9 / 10$ of the air load was centrifugally supported.

It should be pointed out that this 2.44 meter ( $8 \mathrm{ft}$ ) diameter model rotor does not have coning built in. Thus, centrifugal restoration is solely the result of air load blade deflection. Because these blades are relatively heavy (solid aluminum) and the tip speed is high, appreciable restoring moments are generated with reasonable tip amplitude displacements of 5.1 $\mathrm{cm}$ (2 in.) for the $30 \mathrm{AF}$ blade and $10.2 \mathrm{~cm}$ (4 in.) for the $1.5 \mathrm{AF}$ blade for the luad case with $35.2 \mathrm{~m} / \mathrm{sec}(80 \mathrm{MPH}$ ) wind velocity and $213 \mathrm{~m} / \mathrm{s}$ (700 ft per sec) tip speed.

From the above study it is apparent blade stressing is very sensitive to centrifugal reaction. Other possible blade loading cases were examined where tip speeds may be held down, with centrifugal restoration reduced by the square of rotor RPM and air loading maintained at high levels. A review of blade air loading for these wind turbines shows that blade lift is generated predominately by tip speed rather than the wind velocities. Thus, as tip speed drops off, air loading decreases essentially as a squared effect. The centrifugal restoration will also decrease by the square of tip speed. Blade stressing as shown on figure 7-1 was judged acceptable for the $15 \mathrm{AF}$. blade. It should be noted that cyclic stressing of the blade is not alleviated by centrifugal restoration. If this blade were to be run with skewed inflow, which is not scheduled in the test program, significant stressing can be expected.

In the design of full scale wind turbine blading, light weight blade construction is being considered. Here, since blade mass and tip. speed are down, centrifugal restoration is enhanced by building in coning. As in the case of the model blade, at least for the higher velocity ratio configurations, it appears improbable that low tip speed high lift load cases will damage the blades. However, the converse case should be discussed. If the wind turbine load were lost and the rotor went to free running speed (approximately twice design speed) blade lift loads would not be able to cancel the centrifugal restoration loads. The blade may be overstressed as the centrifugal effect tends to reduce the built-in cone angle.

From simplified relations the torsional frequency of the 15 AF relative to the $30 \mathrm{AF}$ blade of equal length and one half chord and thickness is:

$$
\begin{aligned}
& w=f\left[\frac{I_{\mathrm{P}}^{4}}{\mathrm{w}}\right]^{1 / 2}=f\left[\frac{(\text { thickness })^{3} \text { (chord) }}{(\text { chord })(\text { thickness })}\right]^{1 / 2} \\
& \mathrm{w}=\mathrm{f}[\text { thickness }]
\end{aligned}
$$

Thus the scaled $15 \mathrm{AF}$ blade of equal length would be expected to have $1 / 2$ the torsional frequency of the $30 \mathrm{AF}$ blade.

When this frequency is applied to the classical torsional stall flutter relations and compared to empirical data from full scale propeller blade designs, the $15 \mathrm{AF}$ blade as sçaled from the $30 \mathrm{AF}$ is inadequate. Consequently, the blade thickness parameters were extensively modified to 
increase the blade torsional frequency and simultaneously maintain a low bending frequency.

A series of these iterations and their resultant effect on torsional and bending frequencies is shown below. The more interesting variations in thickness distribution are shown in figure 7-5.

\begin{tabular}{|c|c|c|c|}
\hline Run & Description & $\begin{array}{l}\text { wise Bending } \\
\text { Mode Crossover }\end{array}$ & $\begin{array}{l}\text { Stall Flutter } \\
\text { Parameter }\end{array}$ \\
\hline 1 & $\begin{array}{l}\text { Base blade } 30 \text { AF thick/chord } \\
\text { distribution }\end{array}$ & 869 & . 386 \\
\hline 2 & $10 \%$ thickened in mid sections & 955 & .421 \\
\hline 3 & $20 \%$ thickened in mid sections & 963 & .447 \\
\hline 4 & $50 \%$ inc. at . 8 sta. + run (3) & 943 & .451 \\
\hline 5 & $50 \%$ inc. at . 6 sta. + run (3) & 1005 & .468 \\
\hline 6 & $50 \%$ inc. at . 4 sta. + run (3) & 1038 & .452 \\
\hline 7 & Thickened tip on run (1) & 790 & .386 \\
\hline 8 & NASA Mod. 0 thickness/chord ratio & 922 & .458 \\
\hline 9 & NASA Mod. 0 thinned tip & 1017 & .467 \\
\hline 10 & NASA Mod. 0 thinned tip \&.4 sta. & 980 & .461 \\
\hline 11 & Thinned NASA Mod. 0 & 918 & .458 \\
\hline
\end{tabular}

The final thickness distribution is shown on figure 7-2. Again it should be pointed out in the full scale spar shell blade construction these parameters can be iterated against shell and spar thickness as well as airfoil thickness. Also in filament wound blading, fiber orientation can be varied in adjusting bending and torsional frequency placement.

The configuration as defined by run (11) was finally selected as the best configuration for the 15 AF blade. The bending frequency has been properly placed and the stall flutter parameter has been increased to the maximum value within blade thickness ratio restraints. Figure 7-6 defines operating envelope restrictions imposed on the 15 AF blade resulting from the torsional stall flutter parameter of .46. This potential operating boundary is based on empirical data on full scale propeller blades operating near the stall region. These data have been collected over many years of propeller testing. There is a degree of question as to whether propeller derived stall flutter parameters can be used to properly establish wind turbine design configurations. This becomes particularly pressing in the solid model configurations where the fundamental blade bending modes and the torsional mode are lower with respect to the operating speed than has been experienced on full size 
hollow construction. The other factor of major influence is the high tip speed dictated by the aerodynamic test objectives.

Full scale hollow blades will have inherently higher bending and torsional frequencies, equivalent mode separation, and lower tip speeds. Stability analysis conducted during the NASA/G.E./H.S. study program and during the design of the NASA Mod. 0 blades confirm that full-scale turbine blades can be made stable within acceptable weight and geometry restrictions.

The wind turbine model program was run without strain gage monitoring on the blades in order to reduce costs. Thus, the existence of flutter and the extent of pressure resulting blade damage was not experimentally evaluated. To assure a successful aerodynamic test program conservative interpretations and limitations were placed on the test envelope for the 15 AF blade (fig. 7-7).

This figure combines operating limitations resulting from blade bending mode frequency placement and those of the blade, flutter. Two questions obviously arise; 1) Can sufficient test data be collected within the operational limitations to justify testing of this blade? and the second question of, 2) Why push the mechanical blade design to such an extreme?

By testing at fixed freestream velocities and varying the rpm, the complete. performance spectrum of the blade can be developed. This is shown in figure 7-8 where the effect of testing of two different freestream velocities is shown. Thus, the mechanical limitations imposed on this blade design will not inhibit the acquisition of the full performance spectrum of this blade design.

It is recognized that by selecting the $15 \mathrm{AF}$ blade design structural requirements have been extended to the extreme. However, aerodynamic performance data for the low end of practical rotor solidity is of major importance in establishing trends for optimum full-scale wind turbine selection.

\section{$7.4 \quad 60$ ACTIVITY FACTOR BLADE}

The initially selected width/diameter distribution for this blade was the same as the other two blades. However, when assessing manufacturing costs, it became apparent that a significant increase in material and fabrication costs would result due to the wide inboard sections. At that time solid aluminum was also being considered for this blade. A change was made to the planform to provide a constant width inboard section while maintaining the $\mathrm{AF}$ level so that the blade could be made from available $20.4 \mathrm{~cm}$ ( 8 in.) bar stock. Subsequently, manufacturing cost considerations resulted in the selection of an aluminum spar with a wood build up for this blade.

The blade thickness ratio is shown on figure 7-2 and the blade width distribution is shown in figure 7-9. Here, the root, from 40 percent station inward, has been increased in chord length over the 30 activity factor blade. This increased chord length allows for the insertion of the spar member which contains the blade-to-hub attachment. Blade loads are transferred from the wood laminate airfoil sections into the aluminum spar through a fitted taper bonded joint. A backup cross-pin is supplied to prevent blade throwing in a bond failure condition. 
The taper bond joint has been shaped to reduce "tab ending" load buildup conditions at the bond ends. Bond stressing has been kept well under allowables.

Maximum blade bending stresses, figure 7-10, occur in the blade at the 90 percent station. Here, they are well below the yield allowables but not sufficiently to allow cyclic accumulation at skewed inflow operation. Aluminum spar stresses are low in that spar sizing is primarily a result of bond area and load transfer distribution requirements.

The 60 activity factor $230 \mathrm{XX}$ and $44 \mathrm{XX}$ airfoil blades, which are constructed of wood laminate with aluminum spars, have critical speed placements of two-per-revolution and three-per-revolution first flatwise modes above the operational range (figure 7-11). This mode. placement results from the stiffness of the thick root sections in the blade.

Following the structural design of these models the drafting activity was undertaken to provide detailed data for manufacturing of the blades. The major portion of this activity was accomplished on Hamilton Standard's I.D.S. (Interactive Design System). This computer controlled system receives the design definition of airfoils at discrete locations along the blade and automatically splines (fairs) them chordwise and spanwise and also stacks them to the design specification. In the case of these model blades the sections are stacked on the section centers of gravity. The stacked views of the 15,30 , and $60 \mathrm{AF}$ blades are shown in figures 7-12 and 7-13. The system also scribes aluminum plates for the making of blade templates and draws airfoil E.M.D.'s (Engineering Master Drawings) on Mylar for use in checking the finished templates.

In the design of the model blades, consideration was given to full scale applications. One cost effective method of making full scale blades is a filament winding process utilizing a reusable mandrel. The blade stacking shown in figures 7-12 and 7-13 indicates that a mandrel could be utilized in fabricating this blade design configuration and can be withdrawn. This is a result of carefully tailoring blade twist, chord, and thickness parameters on the inboard section. This tailoring has allowed for mandrel removal with minor effect on performance of blade inboard sections. 
MODEL WIND TURBINE BLADES

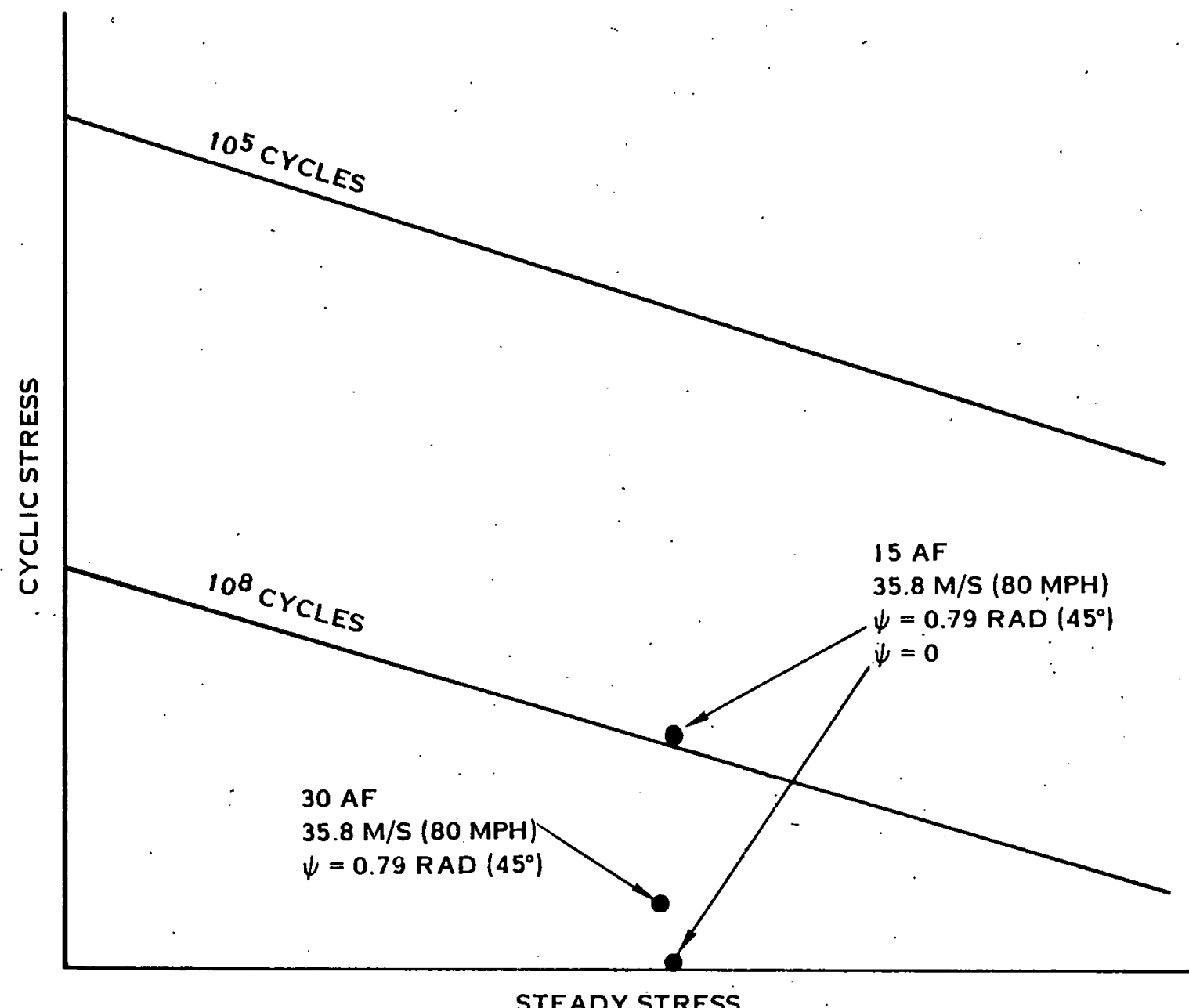

FIGURE 7-1. STRESS-STRENGTH COMPARISON 


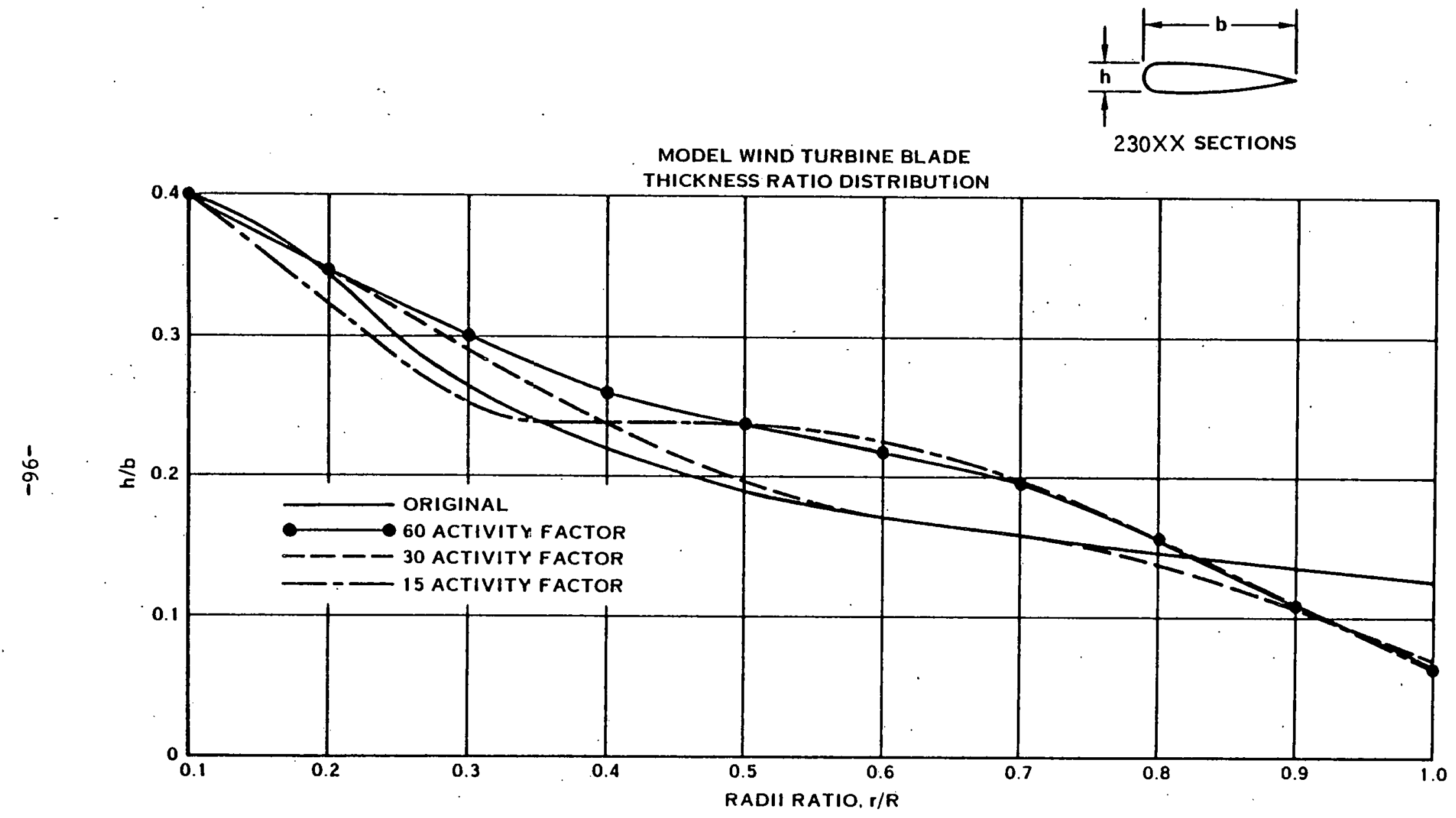

FIGURE 7-2. DESIGN REVISIONS OF THICKNESS DISTRIBUTION 


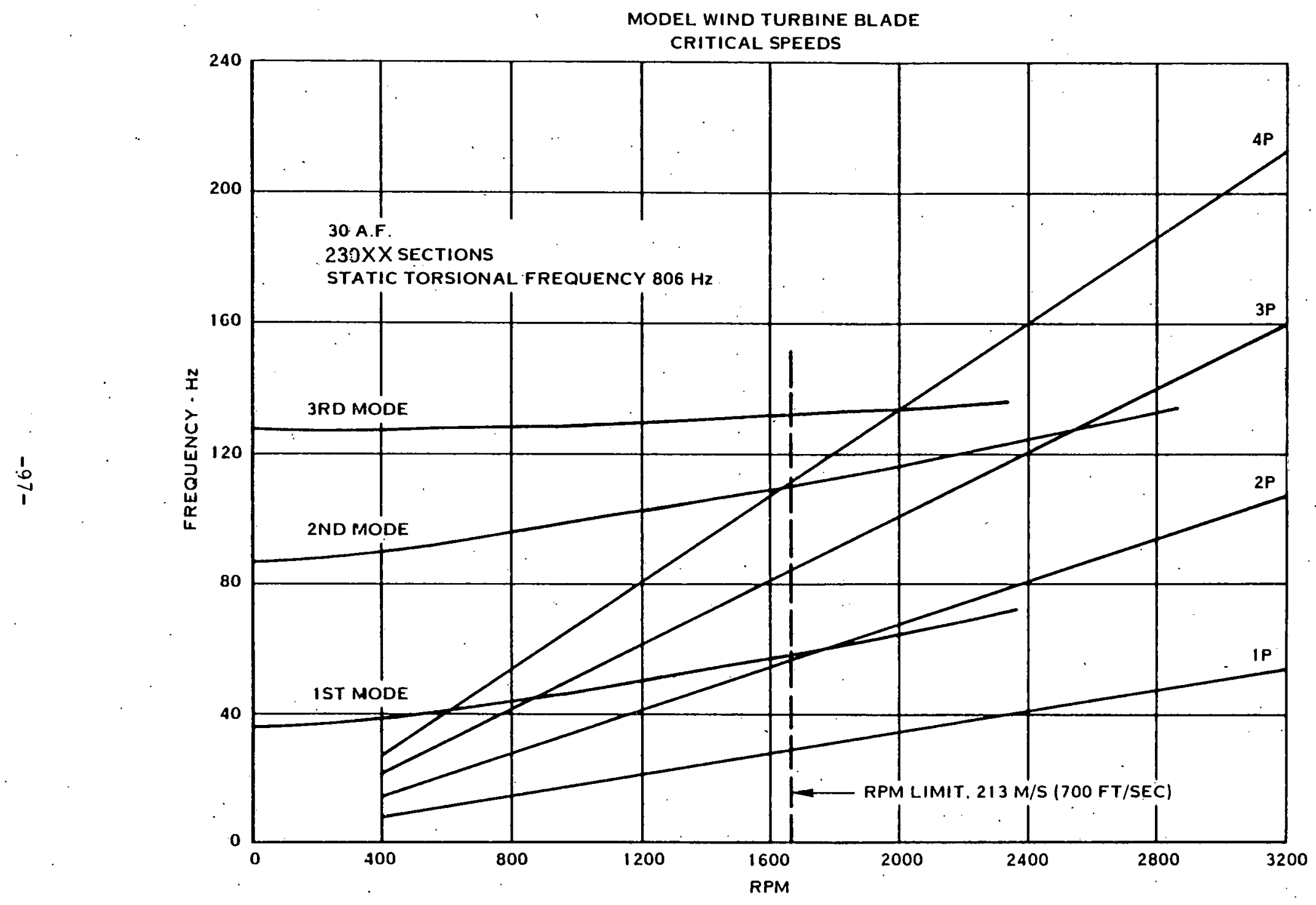

FIGURE 7-3. CRITICAL SPEED DIAGRAM - 30 A.F. 
MODEL WIND TURBINE BLADE

CRITICAL SPEEDS

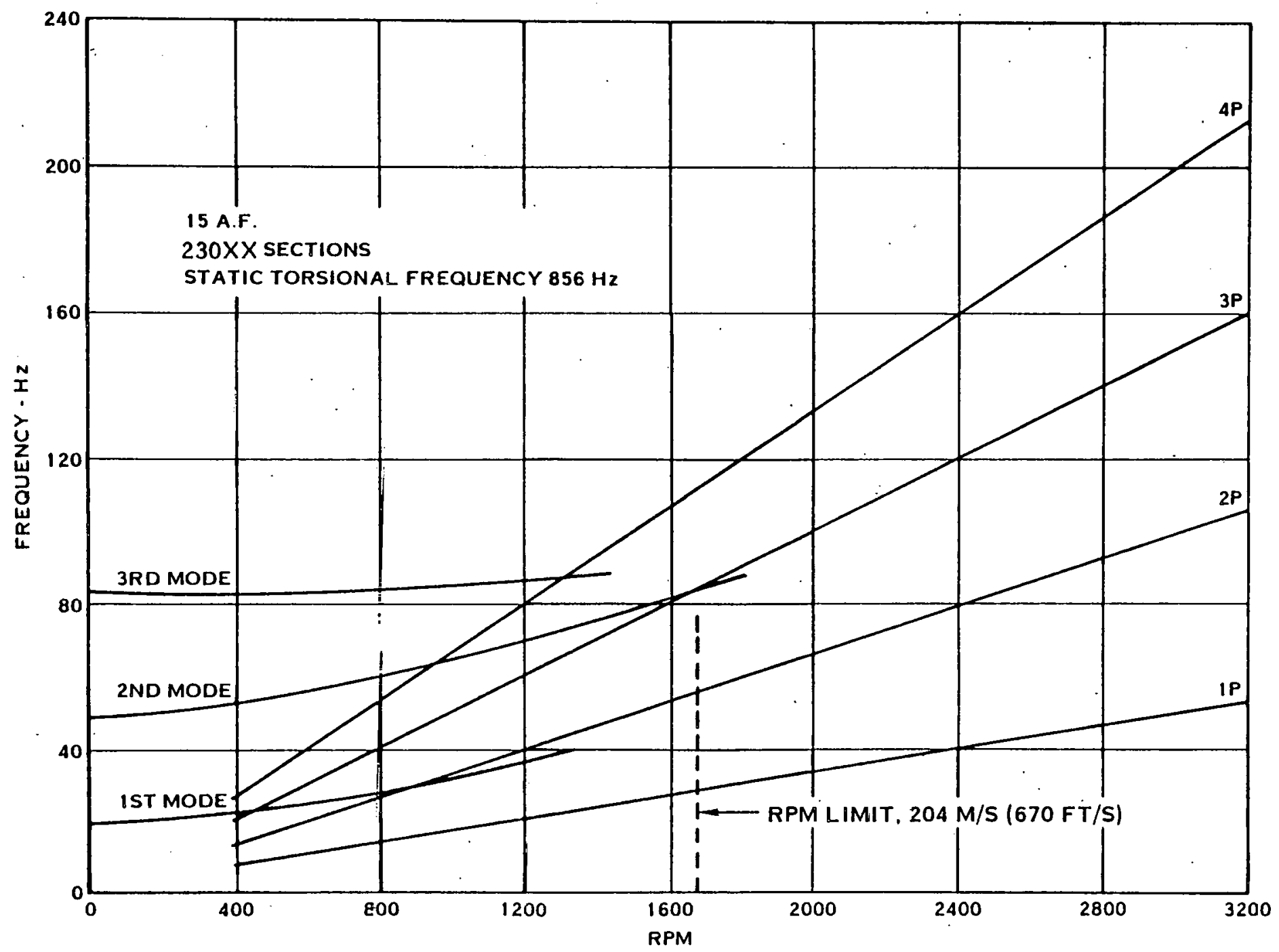

FIGURE 7-4. CRITICAL SPEED DIAGRAM - 15 A.F. 
MODEL WIND TURBINE BLADE

15 A.F.

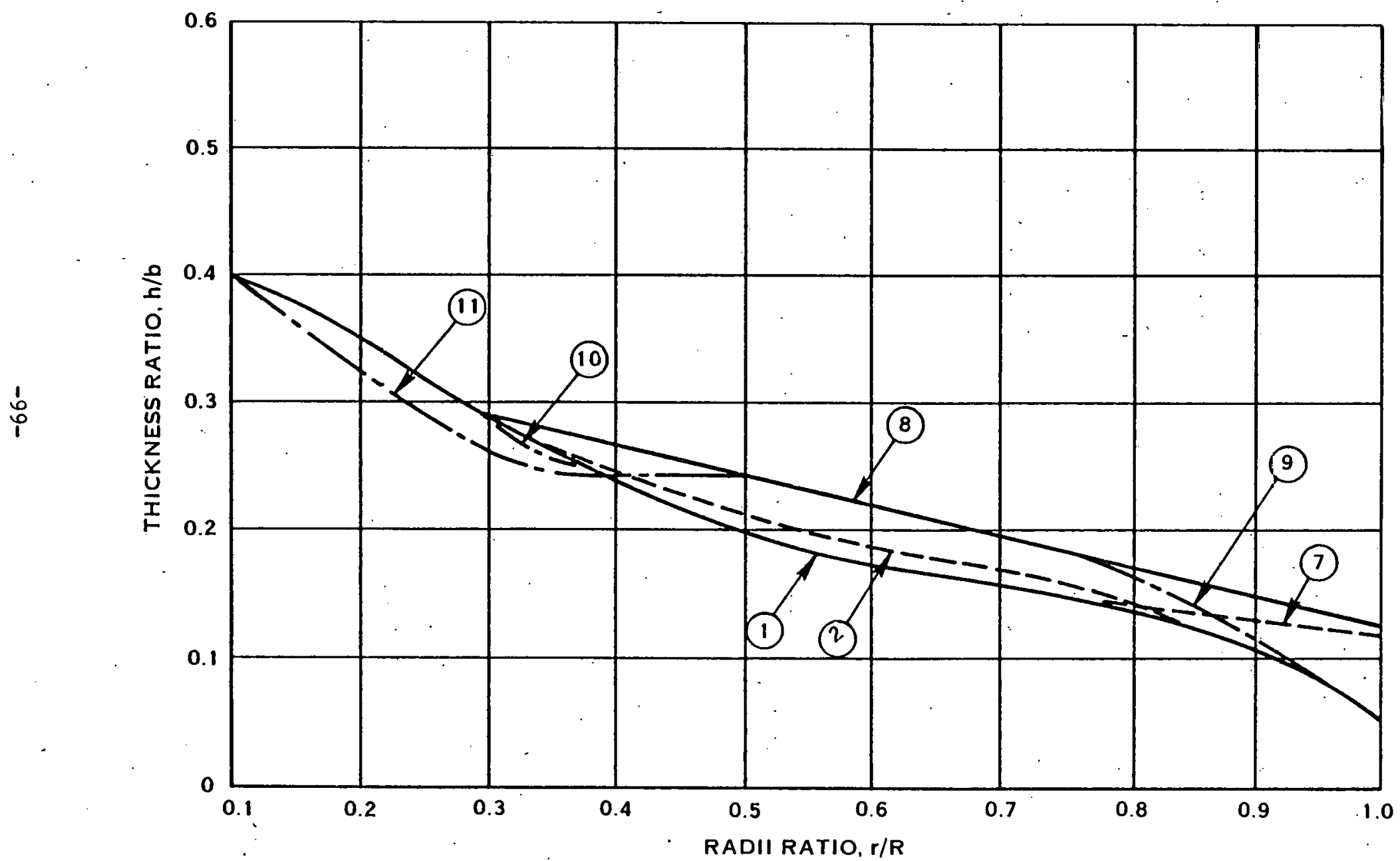

FIGURE 7-5. RANGE OF THICKNESS VARIATIONS 


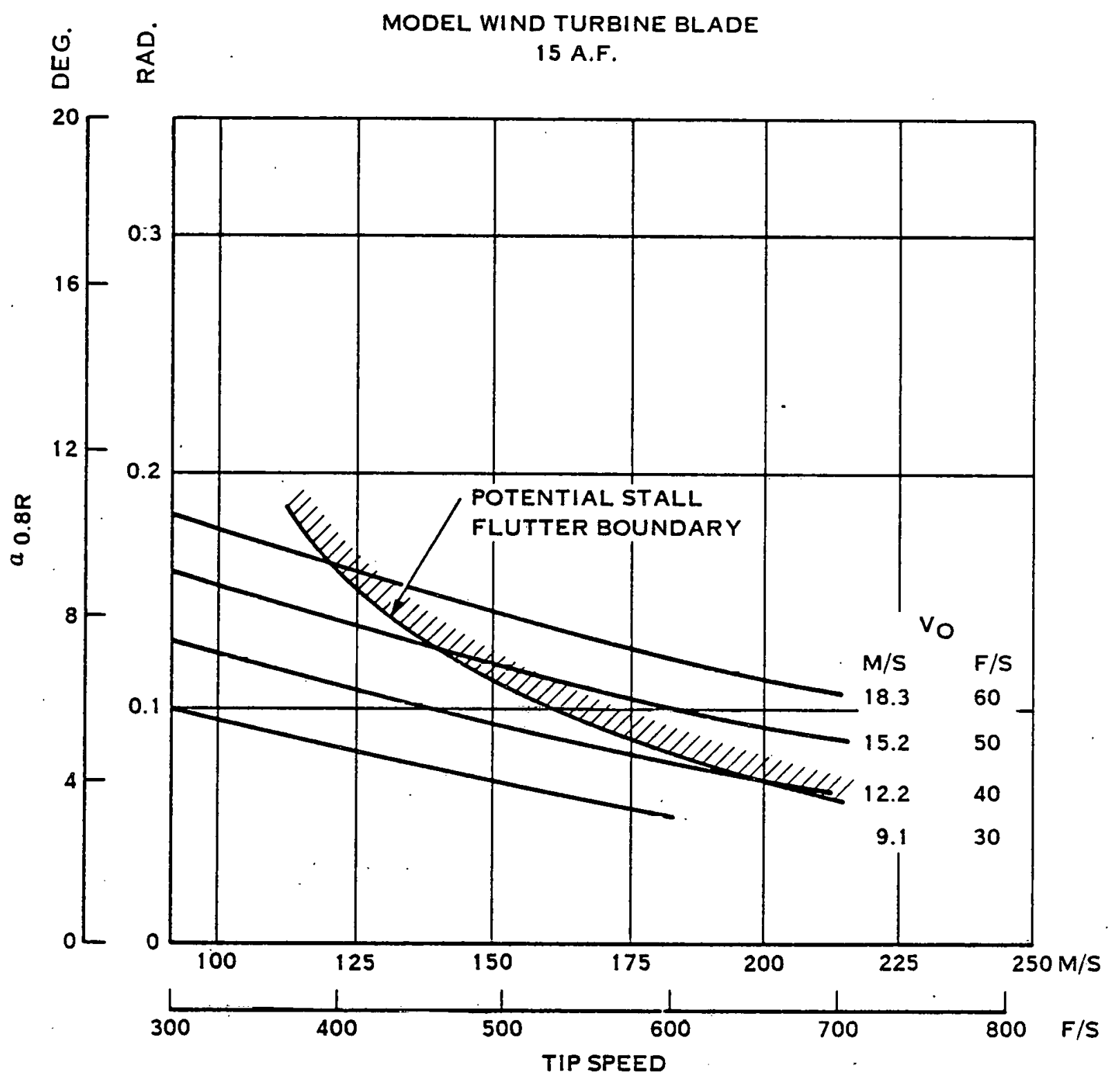

FIGURE 7-6. STALL FLUTTER LIMITATION' 


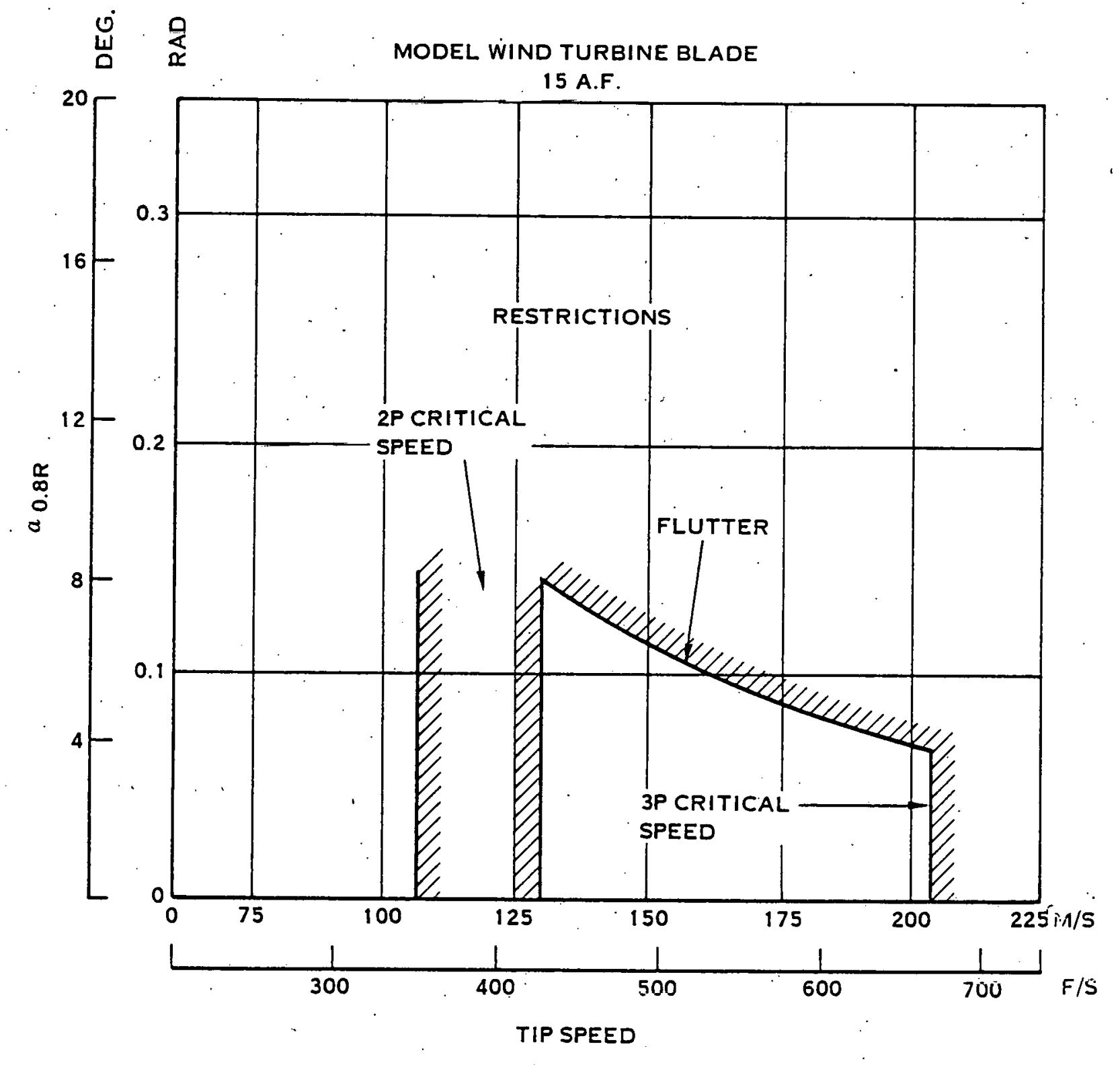

FIGURE 7-7. OPERATING BOUNDARIES 
BLADE ACTIVITY FACTOR $=15$

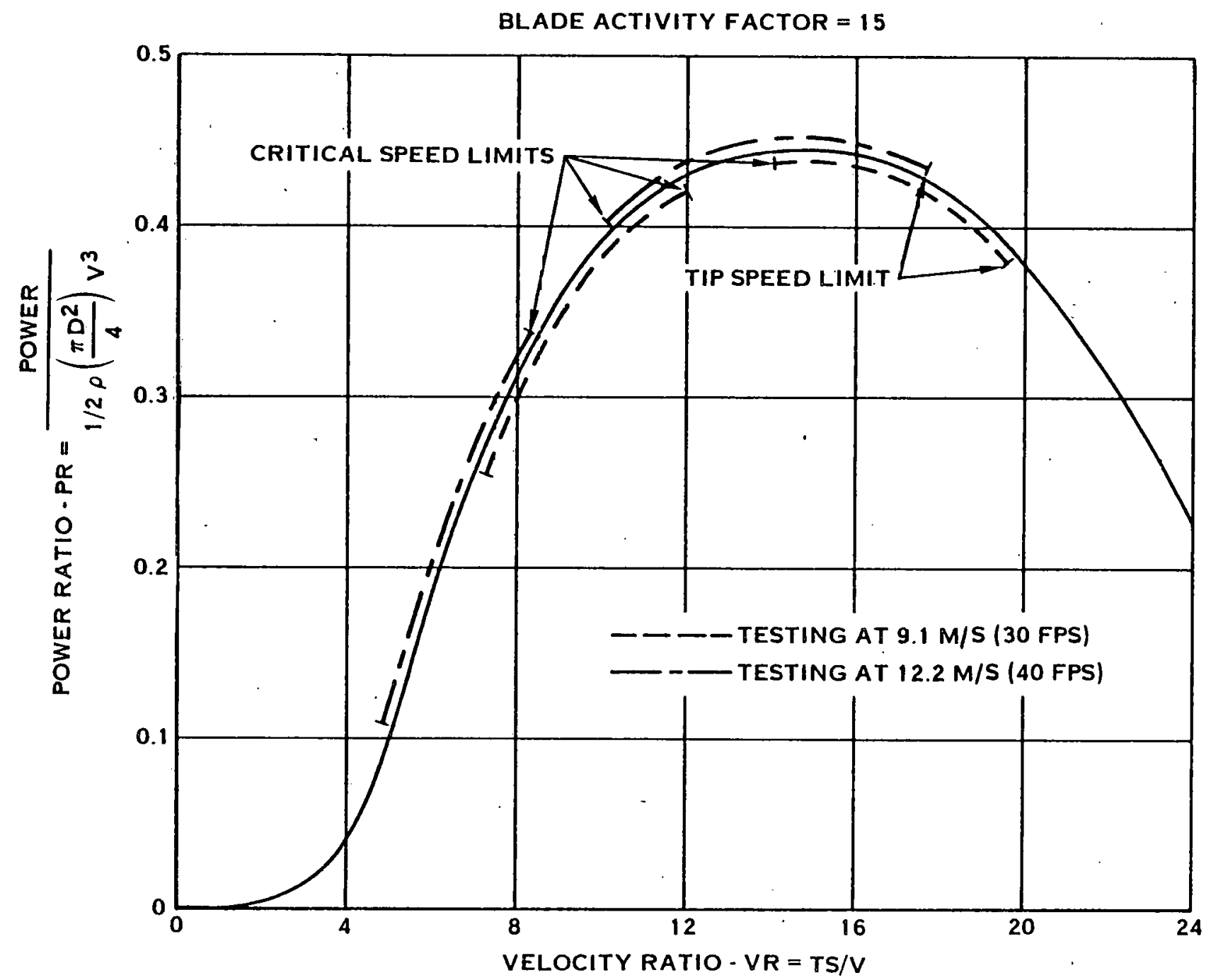

FIGURE 7-8. TURBINE TEST RANGES 


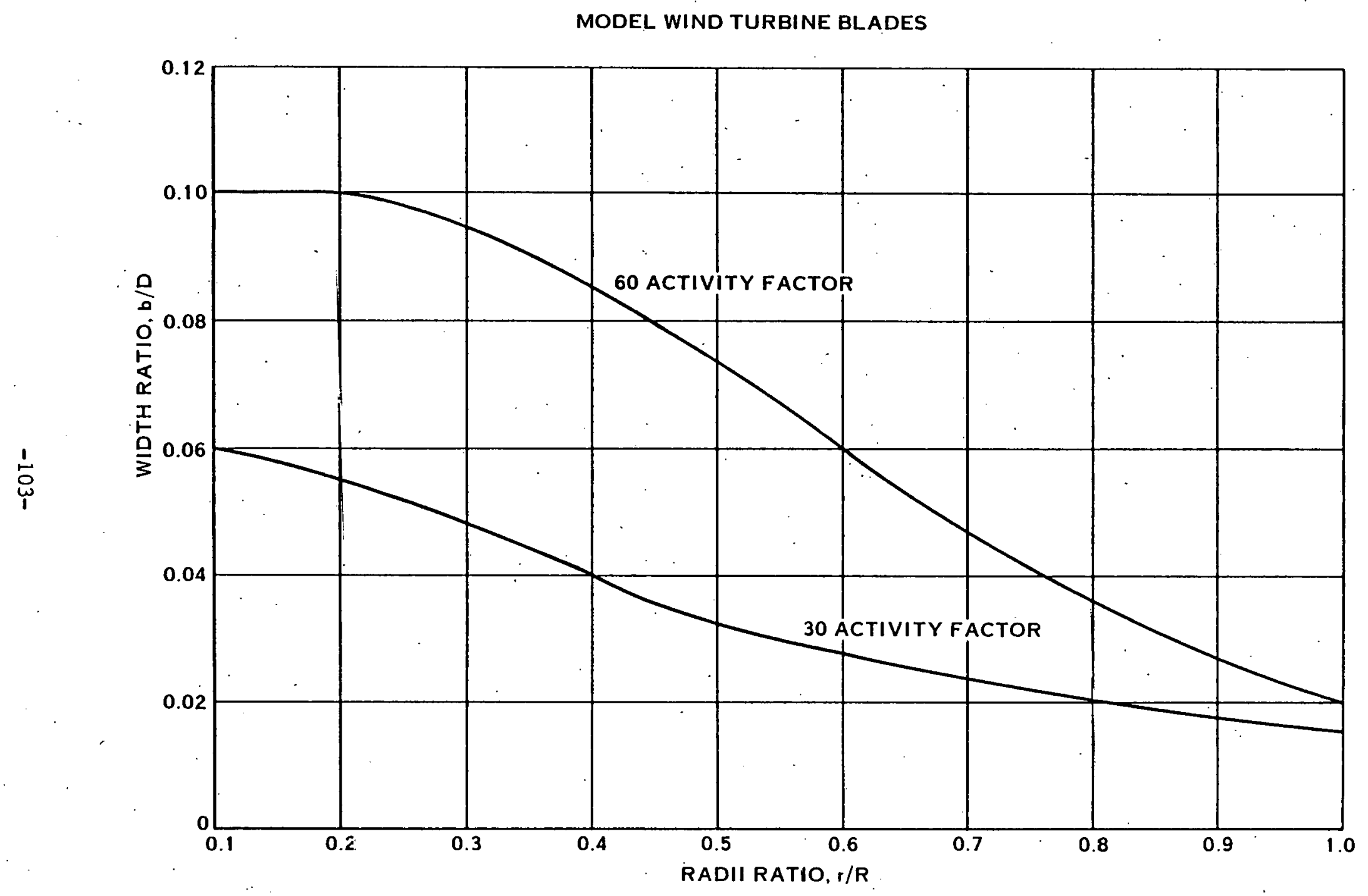

FIGURE 7-9. VARIATION OF WIDTH DISTRIBUTIONS 


\section{BLADE STRESSES}

60 A.F.

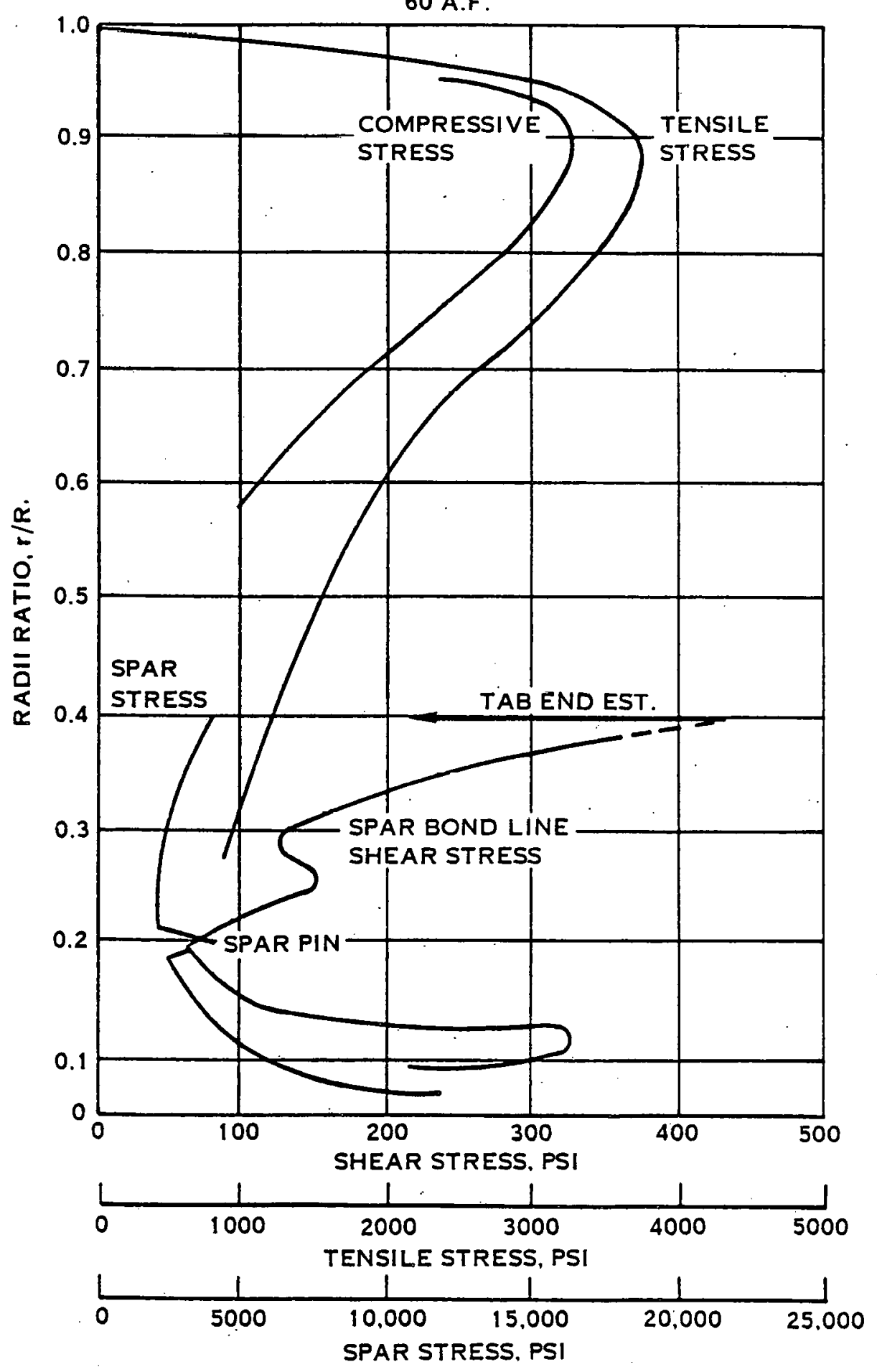

FIGURE 7-10. BLADE AND SPAR STRESSES 


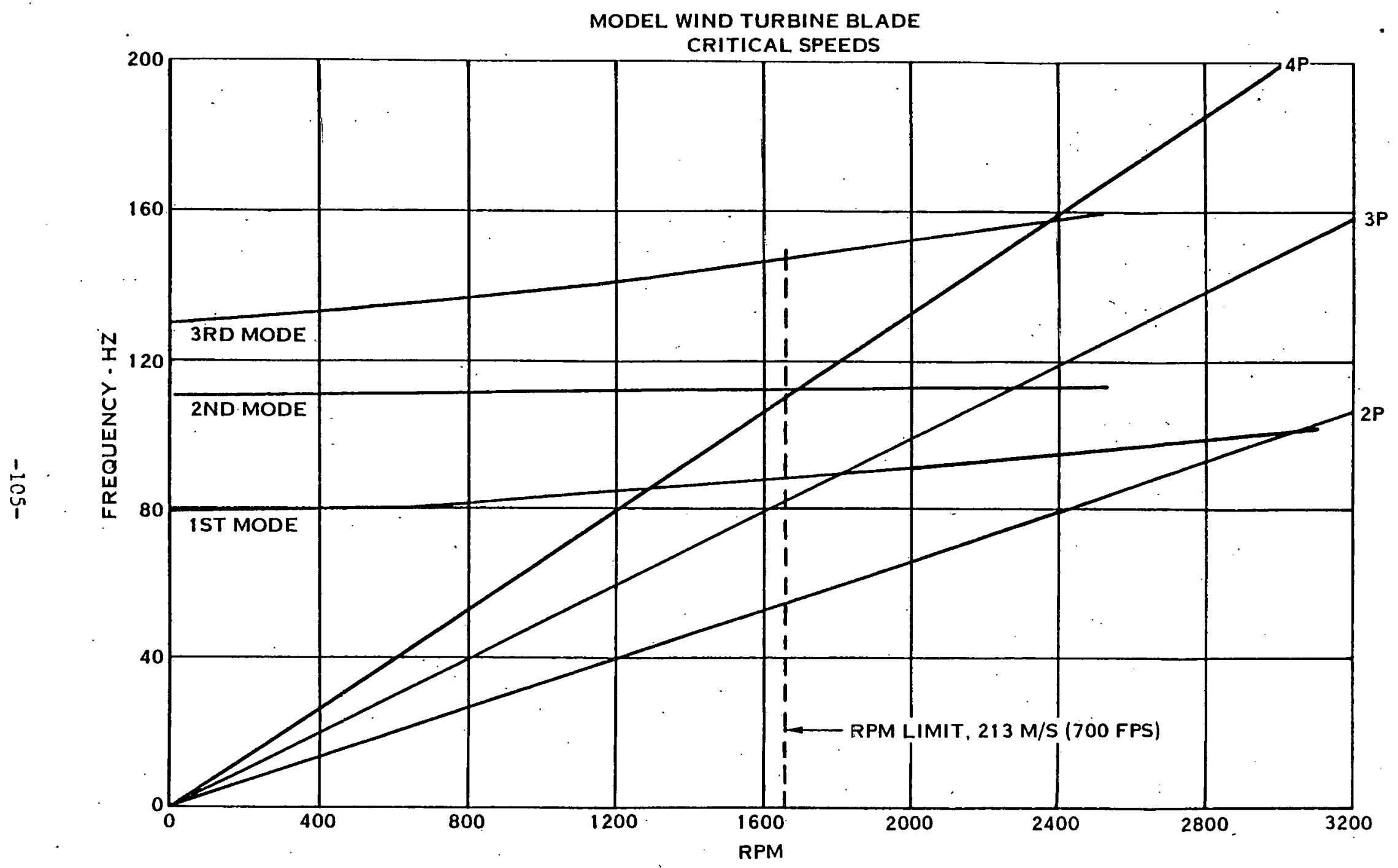

FIGURE 7-11. CRITICAL SPEED DIAGRAM - 60 A.F. 


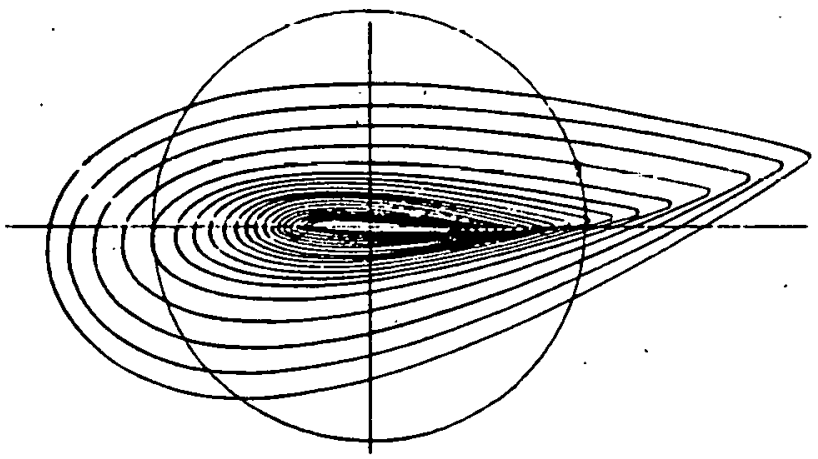

WIND TURB INE BLADE ERDA CDNTRACT E.111-11-2615

PT. NO 765642-2, (I5AF) SCALE .9 TO 1.0

15 A.F.

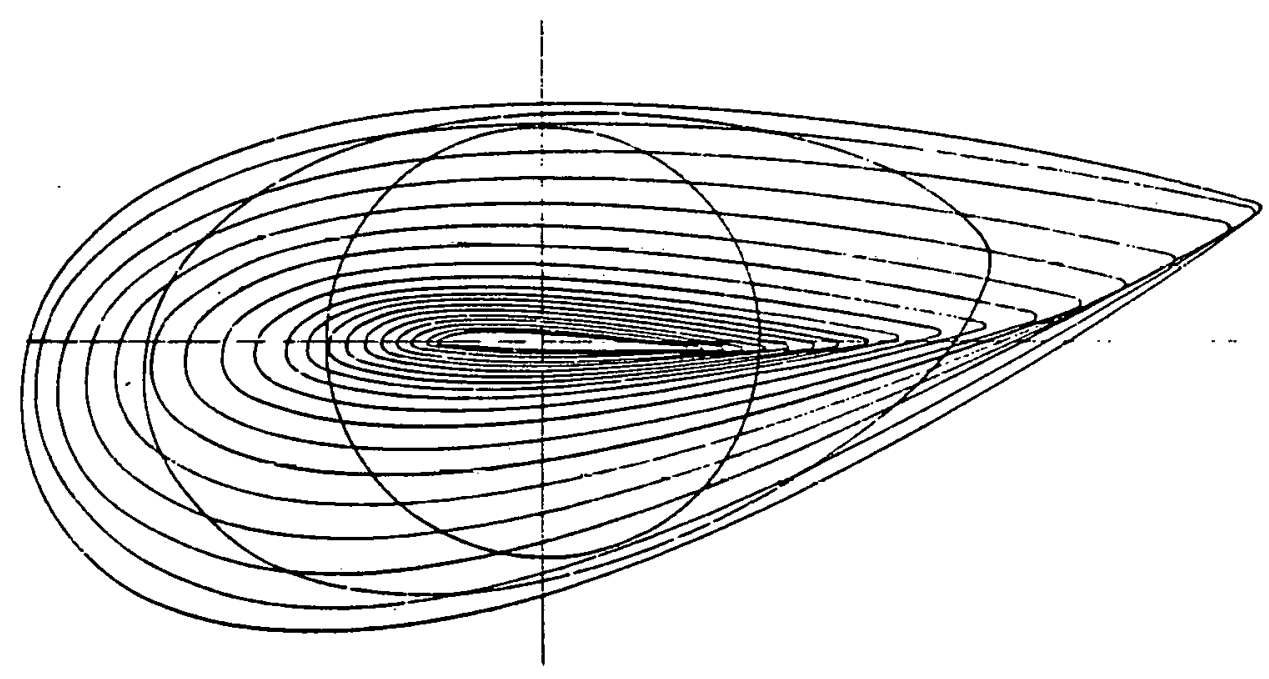

WIND TURB INE BLADE

ERDA 'CDNTRACT E. $111-1)-2615$

PT NO. 765642-1: (3OAF) SCALE. . 9 TO 1.0

30 A.F.

FIGURE 7-12. BLADE AIRFOIL STACKING 


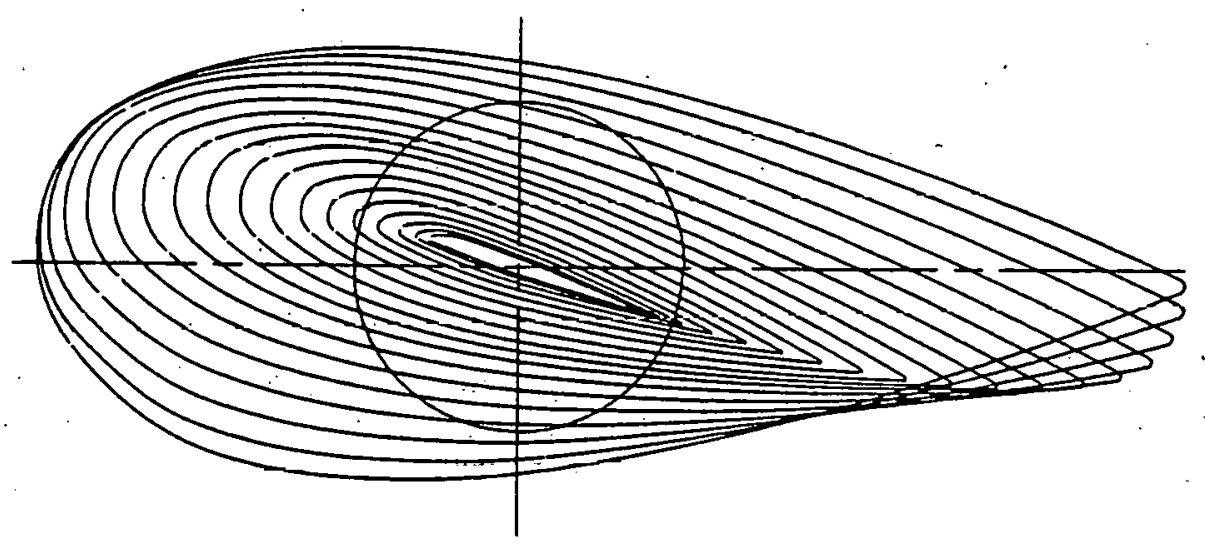

WIND TUREINE BLADE

ERDA CDNTRACT $111 .-11 .-2615$

PT NO $765642-3,160 A F 230001$

$$
\text { SCALE . } 5 \text { TO } 1.0
$$

60 A.F.

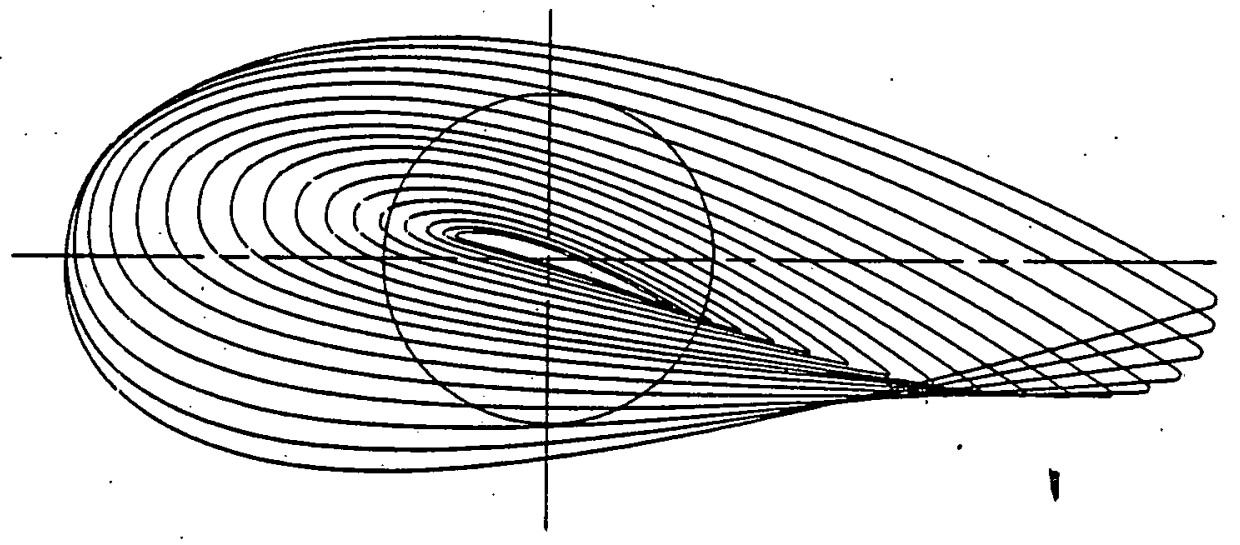

WIND TURB INE BLADE

ERDA CONTRACT $1111-11-2615$

PT NO 765642-4, I SOAF 44001

SCALE. .5 TO 1.0

60 A.F.

FIGURE 7-13. BLADE AIRFOIL STACKING 


\section{SECTION 8}

\section{MANUFACTURE OF MODELS}

It was originally planned that the model blades would be solid metal, probably aluminum, and that they would be machined by a copy mill type process. This process is used by several vendors who are equipped to machine several blades at a time to low dimensional tolerances. This frocess has been used in the past for making small scale propeller models as well as blades for certain full scale flight hardware.

When the model blades were aerodynamically defined and the model diameter selected, preliminary drawings were made and vendors contacted for manufacturing estimates. It was found that the large diameter of the model $(2.4 \mathrm{~m}$; $8 \mathrm{ft}$ ) eliminated several vendors who could not machine blades over .61m (2 ft) in length. Those who could machine this length blade could do so on only one blade at a time. Furthermore, the planform and thickness of the blades resulted in large flexibility which required special support and machining techniques. The cost and schedule estimates received were far in excess of the original estimate.

Consideration was given to reducing the model diameter and it was concluded that if the diameter were reduced to the point where the costs were significantly reduced the usefulness of the aerodynamic data obtained would be seriously impaired.

Accordingly, other materials and fabrication processes were explored. This investigation included blades of metal, all wood layup and molded fiberglass on a metallic spar, and wood layup on a metallic spar. Several sources were investigated for hand machining of the blades. The all wood blades were ruled out because of inadequate torsional stiffness. The fiberglass layup and molding proved too expensive particularly for tooling. The result of this investigation was a decision to make the $15 \mathrm{AF}$ and $30 \mathrm{AF}$ blades of solid aluminum and the larger $60 \mathrm{AF}$ blade of laminated wood laid up on an aluminum spar. All blades will be hand-machined to final shape. This approach resulted in a significant reduction in costs compared to the copy mill type process estimates but still higher cost than that originally estimated.

Consideration was also given to making only two sets of model blades which, after testing, would be reworked to the second two sets of blades.. This option requires two separate test periods to allow time for the rework operations. However, in this case, the wind tunnel setup costs for the second test period nearly offset the cost savings in model manufacture. Moreover, the initial models would not be available for possible rerunning later in the program to check questionable data or future testing which ERDA may want to undertake in the UTRC wind tunnel or in other test facilities. 


\section{SECTION 9}

PREDICTED PERFORMANCE OF THE SELECTED WIND. TURBINE CONFIGURATIONS

\subsection{PREDICTED AERODYNAMIC PERFORMANCE}

The aerodynamic performance of each of six wind turbine models was computed for the complete operating spectrum. The data are presented in the terms of power ratio, P.R., and thrust ratio, T.R., over a velocity ratio, V.R., range from start-up to shutdown and covering a. range of blade angles appropriate for variable pitch power regulation required for the generation of electric a.c. power. with synchronous generator. The performance data presented herein was computed for the same matrix of wind velocities, model rpm's and blade angles as were to be covered in the wind tunnel test.

The modified Goldstein propeller method described in Reference 1 has been utilized to calculate the performance. Thus the test data would provide a check on the accuracy of this method. In this connection it should be pointed out, that while the $2.4 \mathrm{~m}$ ( $8.0 \mathrm{ft}$.) diameter wind turbines appear to be large wind tunnel models, they are in fact small scale relative to the $30-60 \mathrm{~m}(100-200 \mathrm{ft}$.$) diameter range for full$ scale wind turbines currently being considered economically viable machines. Thus while the full scale wind turbines operate in the 3-8 million Reynolds number range, the models in this test program: ware to be operated in the 0.1 to 1.1 million $R_{0} N$. range. As shown in Figure 9-1, for full scale wind turbines, R.N. has only a small effect on performance whereas for model wind turbines the effect of R.N. is significant. Moreover, the variation in R.N. due to differences in activity factor (chord) of several models could result in misleading trends of this parameter when related to full scale wind turbines. Therefore, the test results for this program cannot be used as fuil scale performance. Rather the results can be utilized to check out the methodology which may then be used to predict full scale performance.

Thus to investigate the effect of the blade geometric parameters, it was intended that each model be tested at the wind velocity required to achieve the same Reynolds number at the velocity ratio for peak performance. The predicted performance was computed for the same conditions.

Although for the generation of a.c. electric power with synchronous generators, the rotor rpm is constant which implies essentially constant Reynolds number, the wind tunnel testing technique will be to set. a blade angle and tuntiel velocity and vary rpm by loading the turbine. This procedure results in a variation in R.N. as rpm is changed. Accordingly, the calculated performance has been based on conditions identical to the test including a Reynolds number variation over the velocity ratio range to permit a direct comparison with the test data.

The test schedule on each model was set up to cover several tunnel velocities to define the performance over the entire velocity ratio range and to achieve maximum R.N. at peak power ratio. Three velocities 
were selected for the design blade angle. The first velocity was selected such that the maximum allowable tip speed for $213 \mathrm{~m} / \mathrm{s}$ (700 FPS) be at the free running speed to define the performance over the entire velocity ratio range. The second velocity was selected such that the maximum allowable tip speed occurs at peak P.R. to achieve maximum R.N. at peak power ratio. A third velocity was selected between the first two. Similarly, for off design blade angles, one velocity was selected on the basis of free running speed and another for peak P.R., provided that this velocity did not exceed the velocity limit due to blade structure. The foregoing discussion may best be understood by studying the predicted performance packages for each of the six models included in the test program. These packages incorporate the wind turbine blade shape characteristics, a photograph, and the predicted performance in terms of power ratio and thrust ratio variation with velocity ratio for several blade angles and at several tunnel velocities. Figures 9-2a through 9-2d covers the 2 blade/15 AF blade model, figures 9-3a through 9-3d the 2 blade/30 AF model, figures 9-4a through $9-4 \mathrm{~d}$ the $3 \mathrm{blade} / 30 \mathrm{AF}$ model, figures $9-5 \mathrm{a}$ through $9-5 \mathrm{c}$ the $1 \mathrm{blade} / 30 \mathrm{AF}$ model, figures 9-6a through 9-6d the 2 blade/60 AF/230XX model and figures $9-7 \mathrm{a}$ through $9-7 \mathrm{~d}$ the 2 blade/60 $\Lambda \mathrm{F} / 44 \mathrm{XX}$ model.

\subsection{EFFECT OF INFLOW ON PERFORMANCE}

The 2 blade/ $30 \mathrm{AF}$ model, considered to be the basic or reference model, will be tested at the design blade angle with the plane of the turbine yawed over a range of angles to the tunnel flow. At each yaw angle the six components of moments and forces will be measured. The definition of these forces and moments are presented in figure 9-8a. The corresponding predicted data are presented in figures $9-8 \mathrm{~b}$ through $9-8 \mathrm{~g}$. The power ratio and thrust ratio data were calculated by the Goldstein program for several wind velocities as described previously. The remaining components caused by the inflow were calculated with the Skewed Wake Program: An examination of the figures indicates rather irregular trends in some cases which may be due to the variation in the airfoil data with the very low values of R.N. However, no experimental data has been available to correlate with the predicted values. Thus the test data from this test will provide a check on the validity of these predictions.

\subsection{EFFECT OF REYNOLDS NUMBER ON THE PERFORMANCE UF 'IHE 2 BLADE/ 30 ACTIVITI} FACTOR MODEL

The large effect of Reynolds number on the performance levels of the test models has been discussed previously. As was pointed out, this parameter will have a dominant influence on the performance of each model and on the effect of the geometric parameters. Thus the performance of each model has been calculated. using airfoil data for the appropriate R.N. at each radial station along the blade with variation in wind velocity and rpm corresponding to the testing procedure discussed in Section 10. As an example, the Reynolds number at the blade 75 percent radius for the $2 \mathrm{blade} / 30 \mathrm{AF}$ model can be selected from figure $9-9 \mathrm{a}$ as a function of velocity ratio and wind velocity with a model blade structural limiting tip speed of $213 \mathrm{~m} / \mathrm{s}\left(700 / / \mathrm{sec}_{\text {.) }}\right.$. Figure $9-9 \mathrm{~b}$ shows a plot of power ratio at the design blade angle for an extended range of wind velocities to further establish the effect of Reynolds 
number on model performance. Figure $9-9 c$ shows how the power ratio at the design velocity ratio of the 2 blade/15 AF, $30 \mathrm{AF}$ and $60 \mathrm{AF}$ models are expected to vary as a function of R.N. compared to full scale models of these same geometries. This plot again demonstrates the predicted powerful effect of R.N. on the performance of the test models.

\subsection{EFFECT OF GEOMETRIC VARIABLES ON PREDICTED PERFORMANCE}

Utilizing the performance packages described in figures 9-2 through 9-8 the effects of number of blades, activity factor and airfoil section on power ratio at the design blade angle for the test models are shown in figures 9-10, 9-11 and 9-12, respectively. Figure 9-10 shows that peak performance decreases and design velocity ratio increases as number of blades is decreased. The corresponding full scale wind turbine predicted performance has been superimposed to show the increased performance level expected with full scale R.N. Figure 9-1l shows similar trend's as activity factor is varied from 60 to 15 . However, in this case the reduction in power ratio level is primarily due to the large reduction in R.N. associated with reduced AF, i.e., the R.N. for the $15 \mathrm{AF}$ model is approximately one fourth of that for the $60 \mathrm{AF}$ model. The superimposed full scale performance indicates very little change in peak power ratio as activity factor is varied.

An examination of figure 9-12 indicates a rather small effect of airfoil section on performance. However, the NACA $44 \mathrm{XX}$ airfoil appears to be a slightly better aerodynamic section that the NACA $230 \mathrm{XX}$ airfoil. According to the figure, this section improves performance near peak power ratio and at lower velocity ratios. However, since most wind power machines are rated at velocity ratios slightly below that for peak P.R., the advantage of the NACA $44 \mathrm{XX}$ airfoil is less than implied by the figure. Moreover, the aft location of maximum camber reduces susceptability to surface roughness.

\subsection{EFFECT OF SURFACE ROUGHNESS ON PERFORMANCE}

The effect of surface roughness on wind turbine performance is very important since to compete with other power producing systems, the wind turbines must be fabricated at minimum cost and have long operating lives in all kinds of atmospheric environments. Thus the tolerances on airfoil shape may need to be relaxed from those required for aircraft application. Moreover, some erosion of the surface may occur with time. The'resulting surface roughness will have a degrading effect on performance as shown typically in figure 9-13 for the 2 bladed/60 AF/230XX airfoil model. Again it should be pointed out that this figure is based on model Reynolds number where the impact of roughness is greater than would be expected in full scale wind turbines. However, this effect will be established by incorporating NASA standard roughness on both $60 \mathrm{AF}$ models with NACA $230 \mathrm{XX}$ and NACA $44 \mathrm{XX}$ airfoils. 


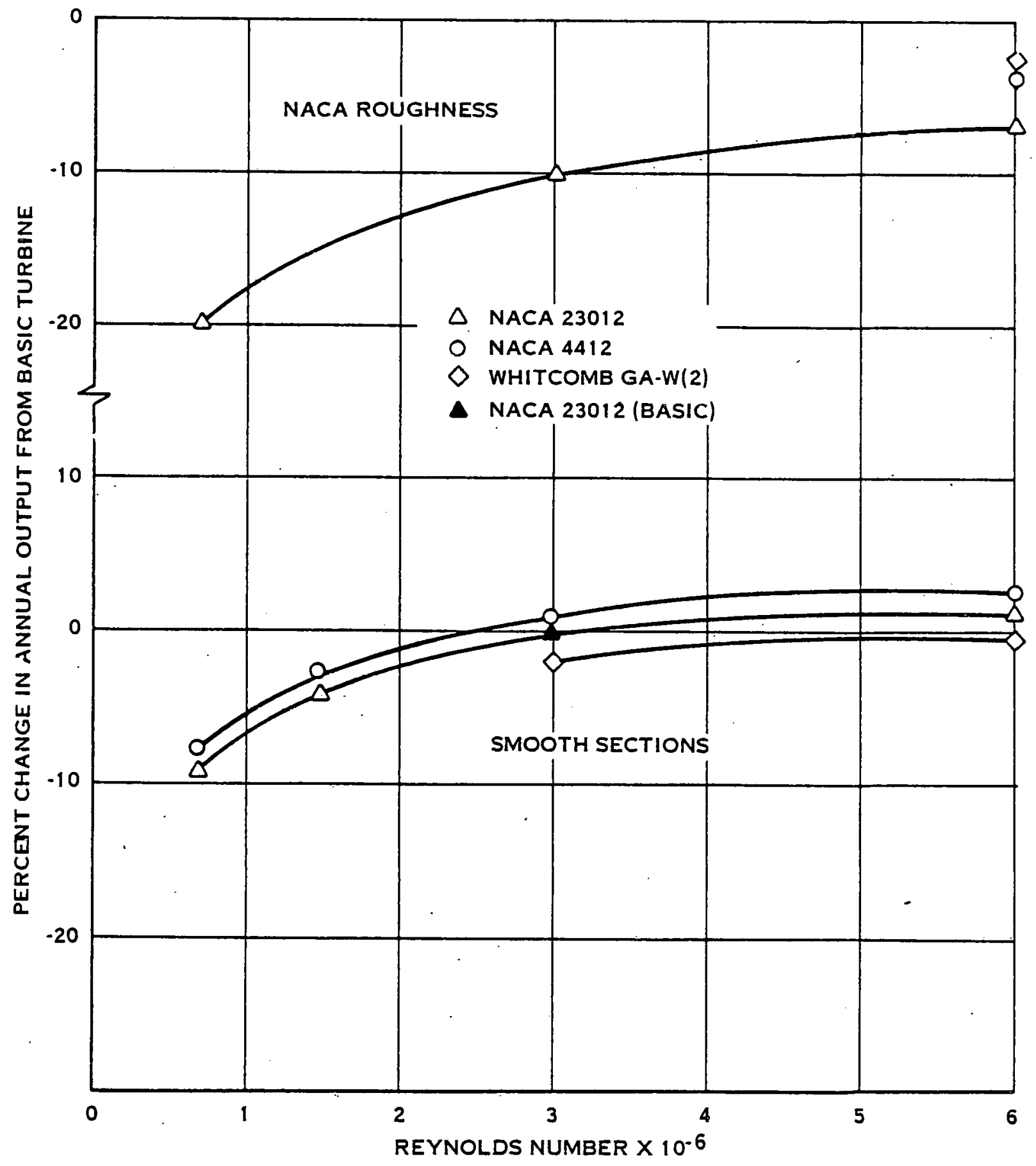

FIGURE 9.1 - EFFECT OF REYNOLDS NUMBER AND ROUGHNESS ON ANNUAL ENERGY OUTPUT OF A $57.9 \mathrm{M}$ (190 FOOT). DIAMETER WIND TURBINE 
MODEL NO. 1

15 ACTIVITY FACTOR

NACA 230XX AIRFOIL SECTIONS

2.4 M (8.0 FT) DIAMETER

2 BLADES

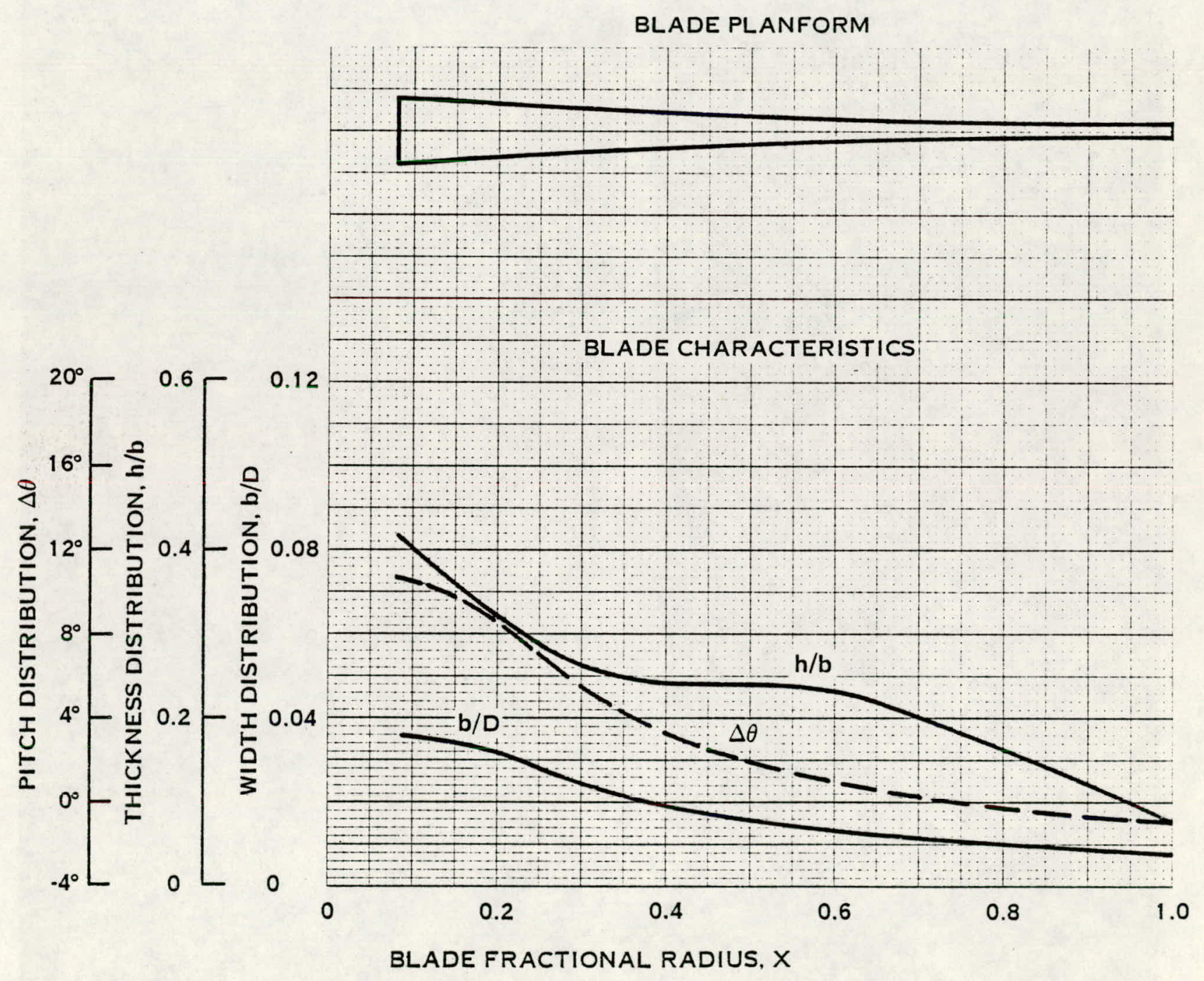

FIGURE 9-2A. BLADE CHARACTERISTICS AND PLANFORM 
MODEL NO. 1

15 ACTIVITY FACTOR

NACA $230 X X$ AIRFOIL SECTIONS

2. $4 \mathrm{M}$ (8.0FT) DIAMETER

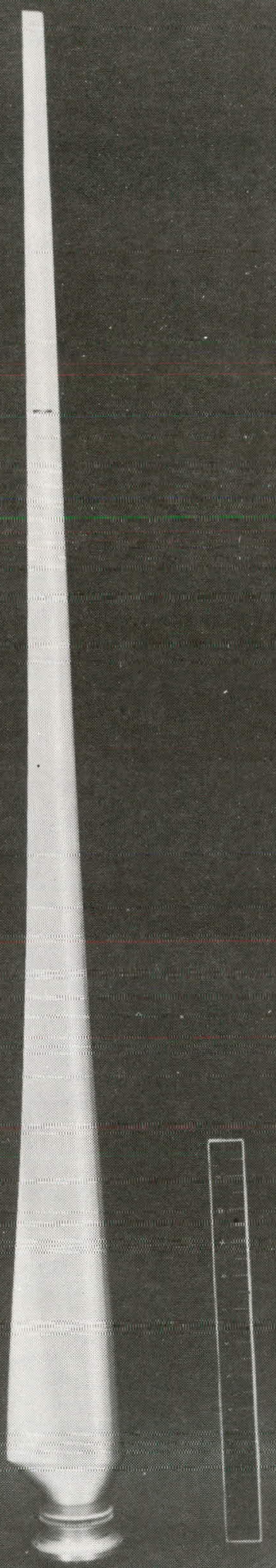

FIGURE 9-2B. BLADE SHAPE 
15 ACTIVITY FACTOR

NACA $230 X X$ AIRFOIL SECTIONS

2 BLADES
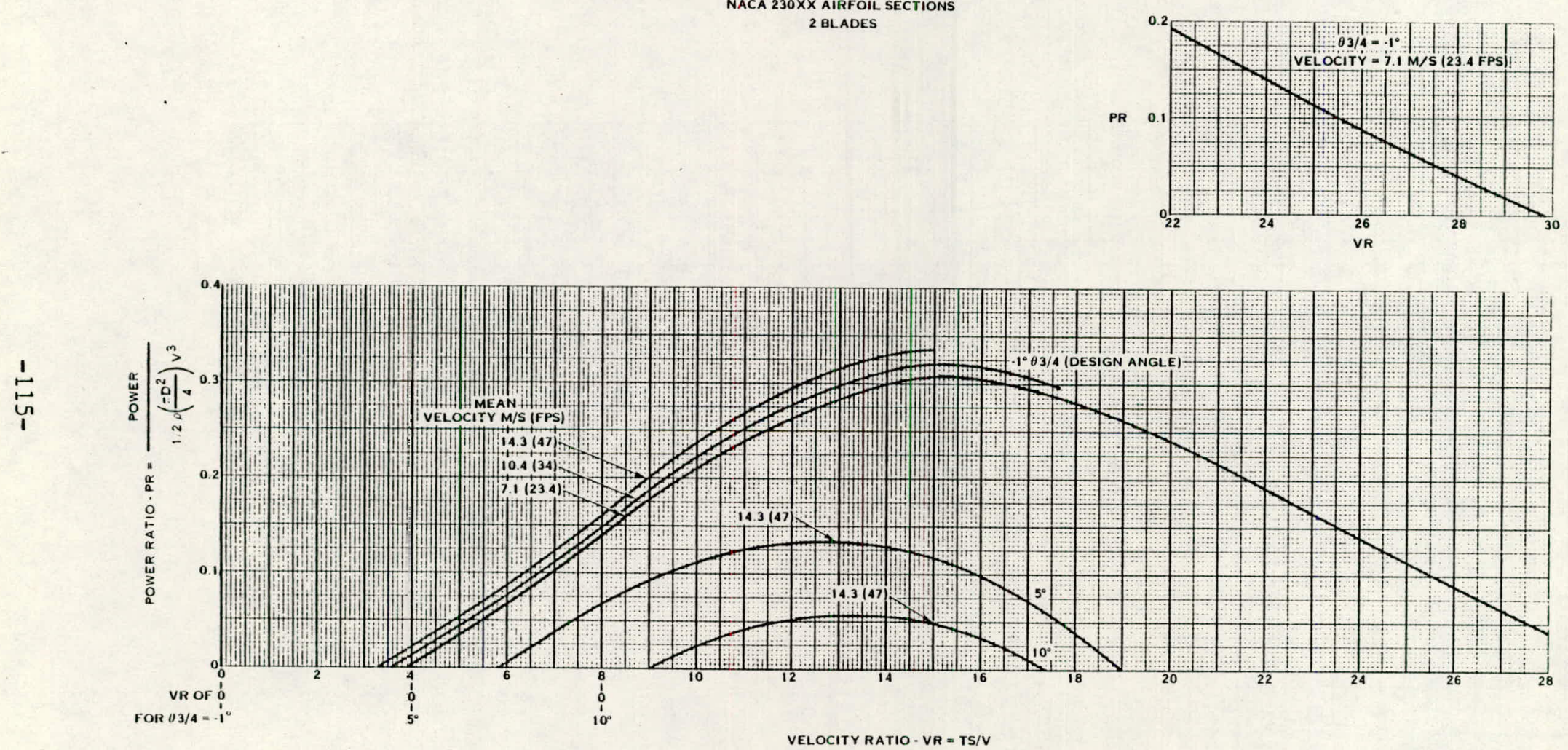

FIGURE 9-2C. EFFECT OF BLADE ANGLE AND MEAN VELOCITY VARIATION ON PREDICTED POWER RATIO 


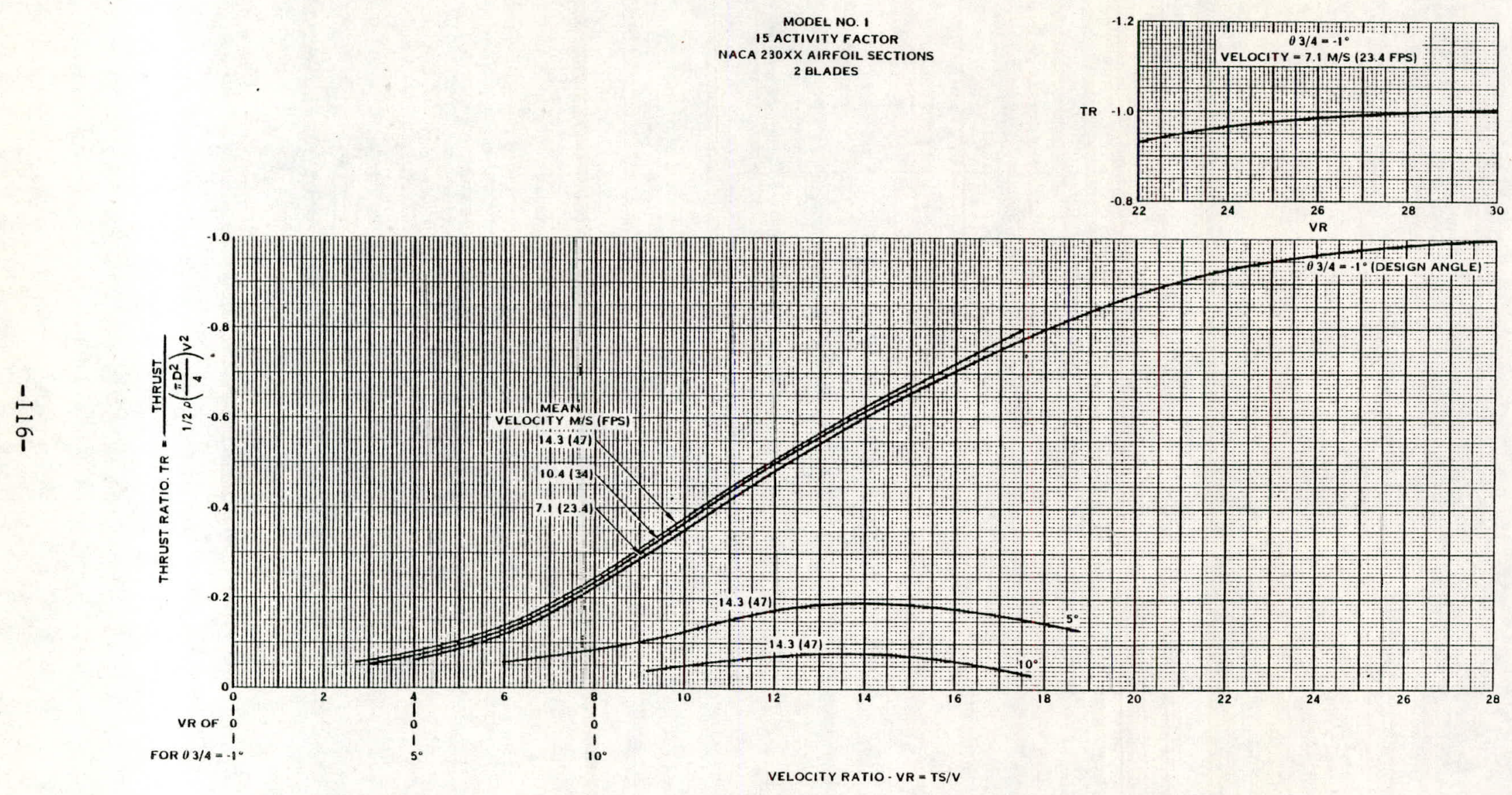

FIGURE 9-2D. EFFECT OF BLADE ANGLE AND MEAN VELOCITY VARIATION ON PREDICTED THRUST RATIO 
MODEL NO. 2 (REFERENCE MODEL)

\section{ACTIVITY FACTOR \\ NACA $230 \times X$ AIRFOIL SECTIONS \\ $2.4 \mathrm{M}$ (8.0 FT) DIAMETER \\ 2 BLADES}

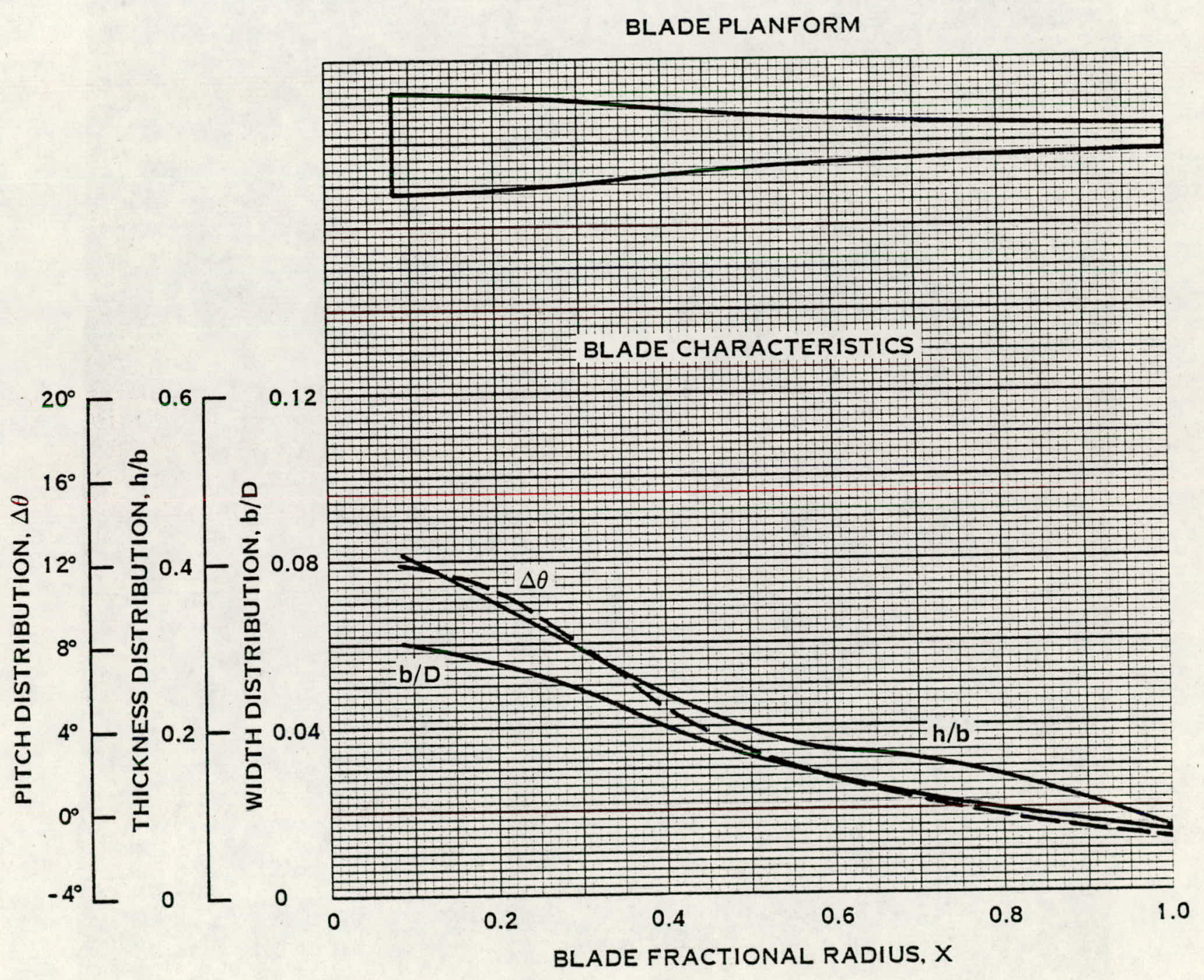

FIGURE 9-3A. BLADE CHARACTERISTICS AND PLANFORM 
MODELS NO. 2,3 , AND 4

30 ACTIVITY FACTOR

NACA $230 \times X$ AIRFOIL SECTIONS

2.4 M (8.0FT) DIAMETER

G 48462

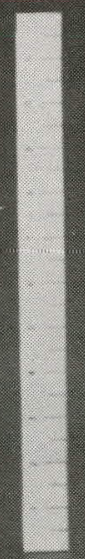

FIGURE 9-3B. BLADE SHAPE 
MODEL NO. 2

30 ACTIVITY FACTOR

NACA $230 X X$ AIRFOIL SECTIONS

2 BLADES

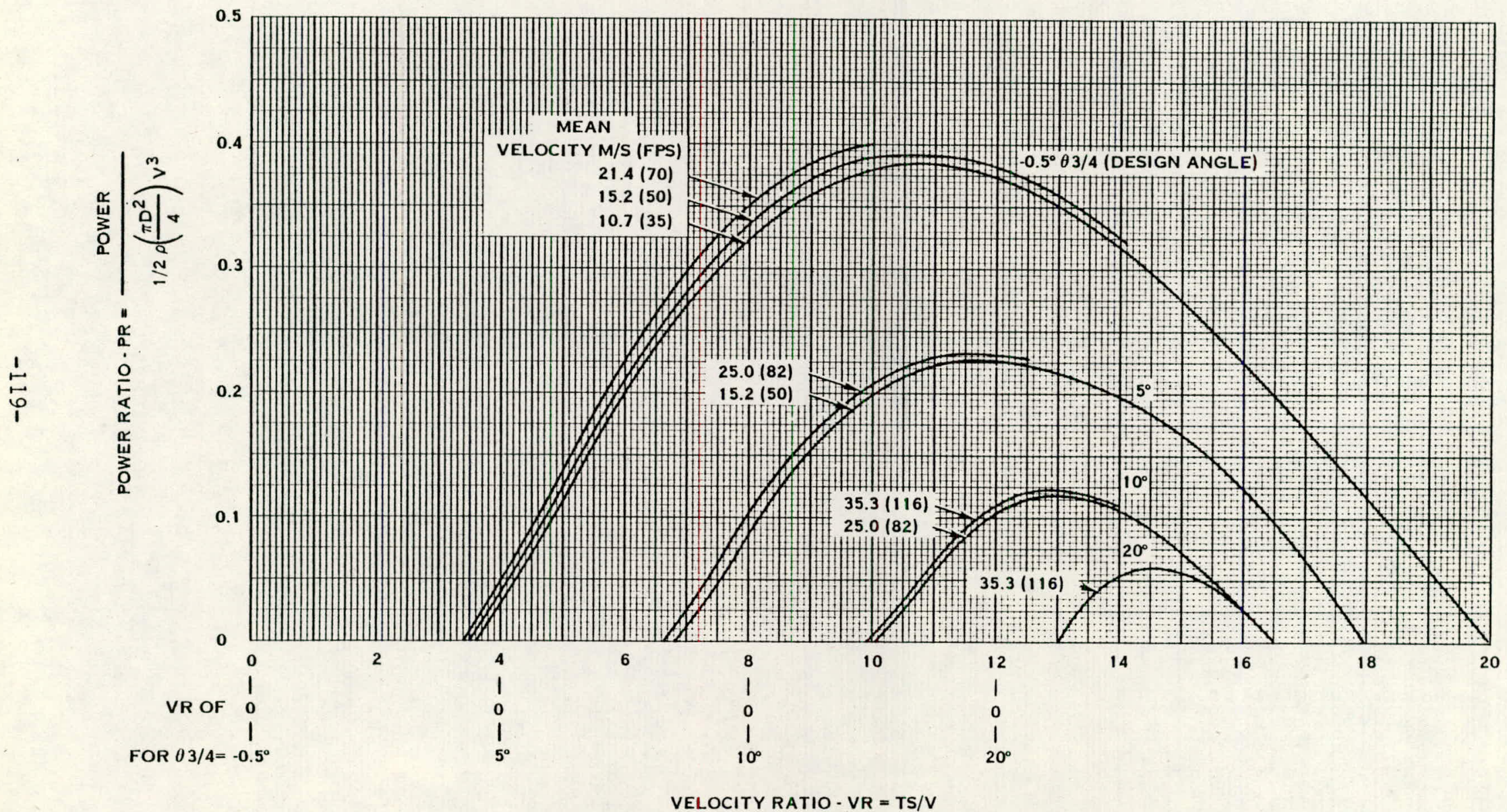

FIGURE 9-3C. EFFECT OF BLADE ANGLE AND MEAN VELOCITY VARIATION ON PREDICTED POWER RATIO 


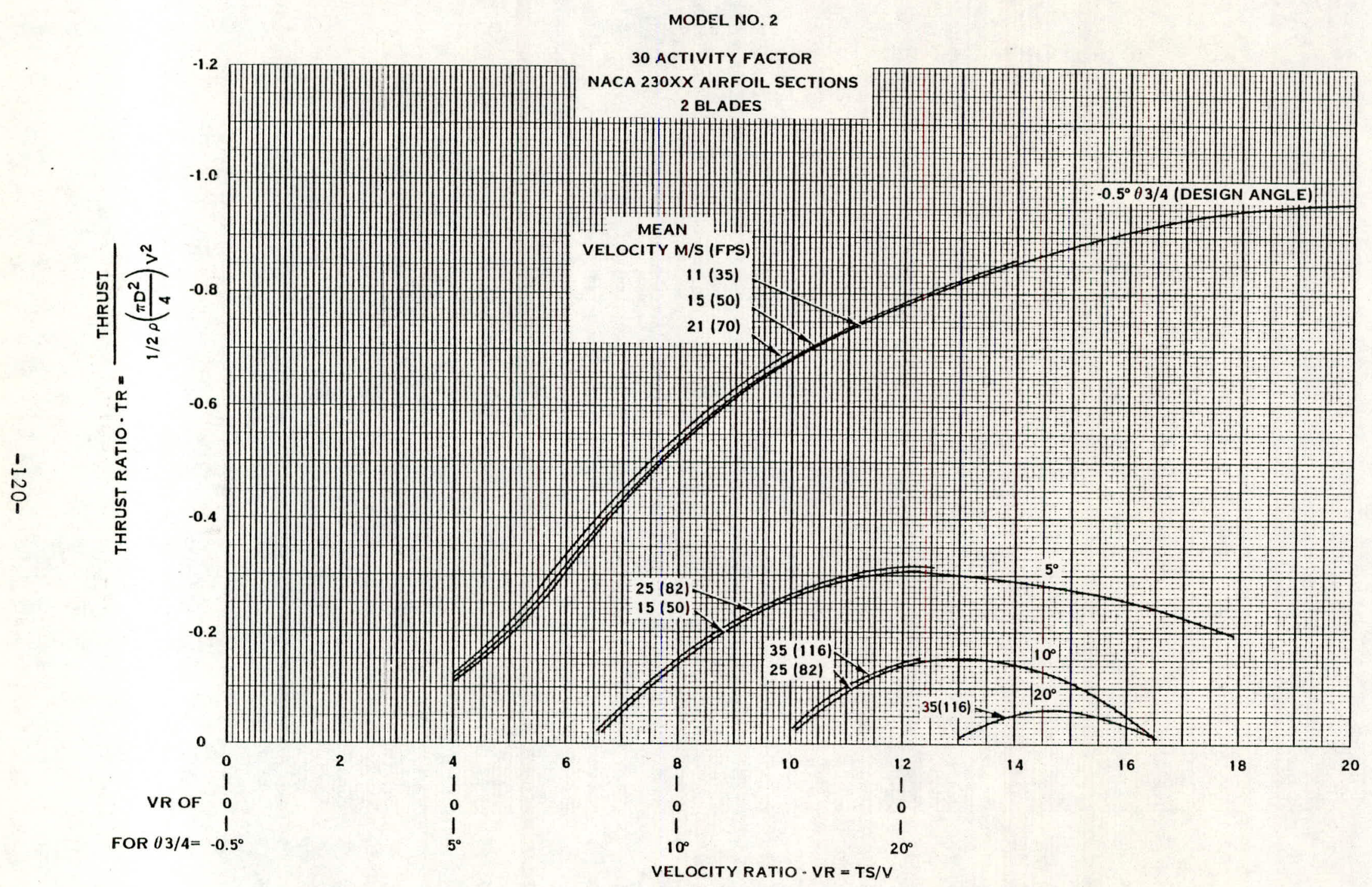

FIGURE 9-3D. EFFECT OF BLADE ANGLE AND MEAN VELOCITY VARIATION ON PREDICTED THRUST RATIO 
MODEL NO. 3

30 ACTIVITY FACTOR

NACA $230 X X$ AIRFOIL SECTIONS

2.4 M (8.0 FT) DIAMETER

3 BLADES

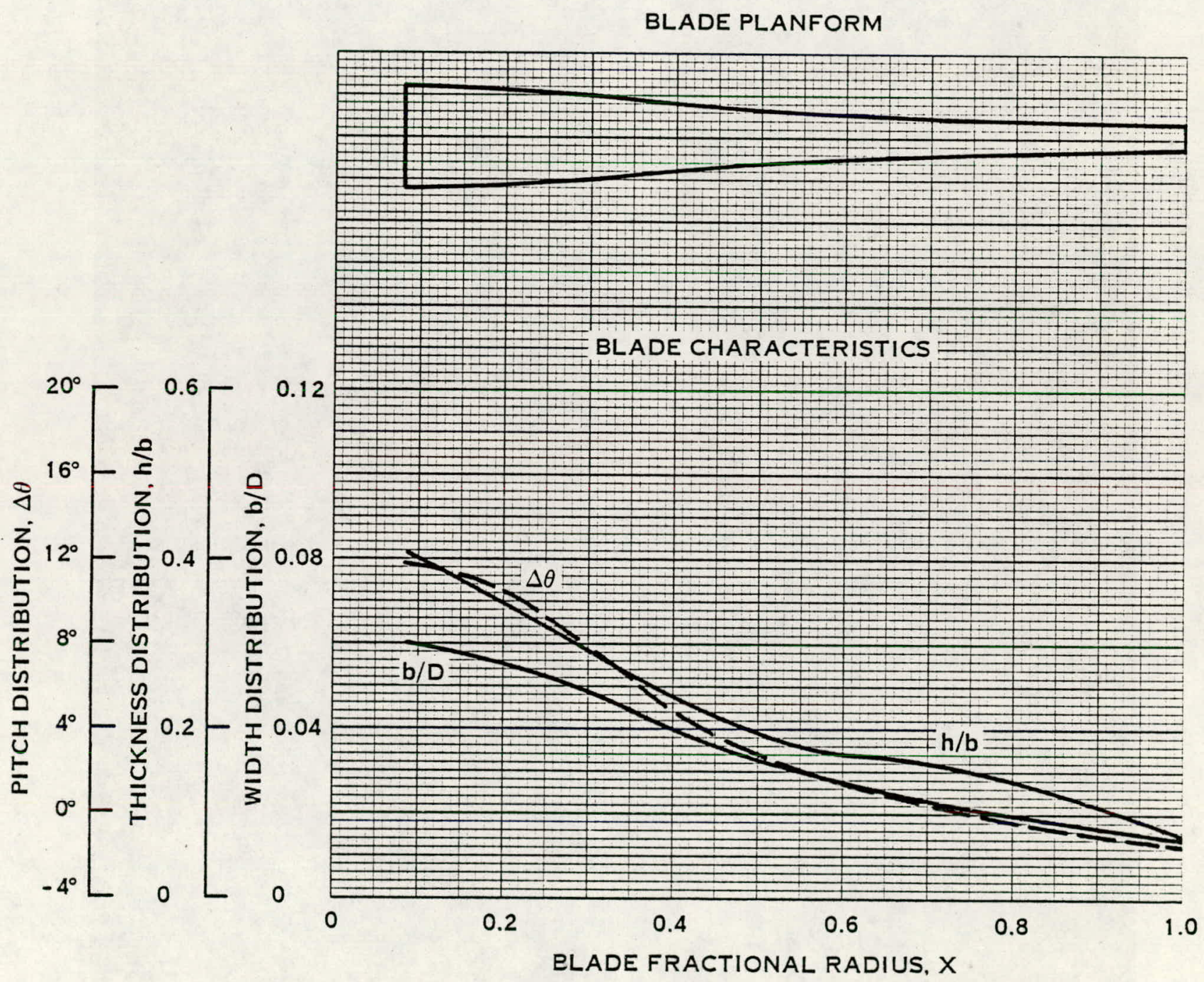

FIGURE 9-4A. BLADE CHARACTERISTICS AND PLANFORM 
MODELS NO. 2, 3, AND 4

30 ACTIVITY FACTOR

NACA $230 \times X$ AIRFOIL SECTIONS

2.4M (8.0FT) DIAMETER

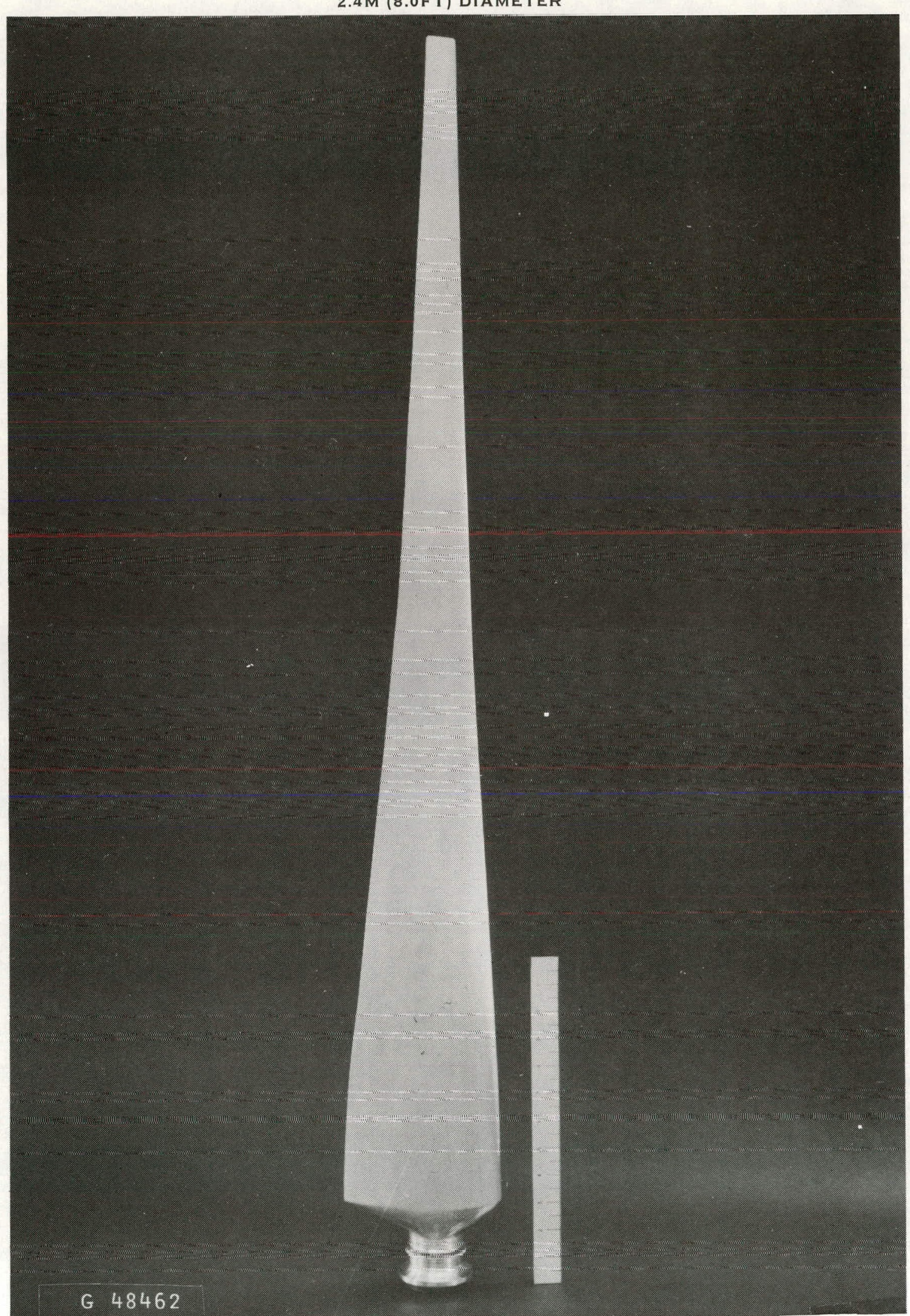

FIGURE 9-4B. BLADE SHAPE 


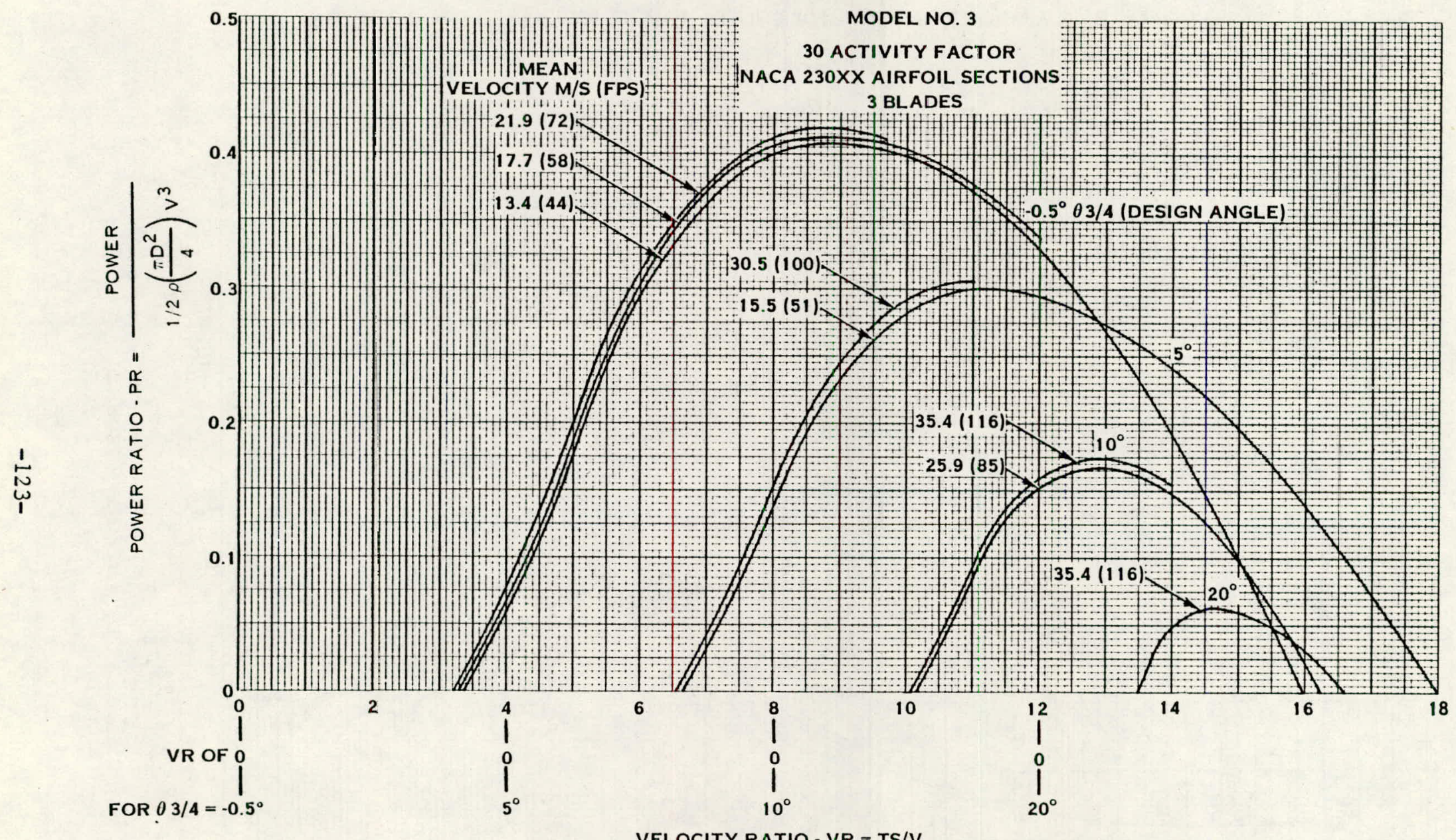

FIGURE 9-4C. EFFECT OF BLADE ANGLE AND MEAN VELOCITY VARIATION ON PREDICTED POWER RATIO 


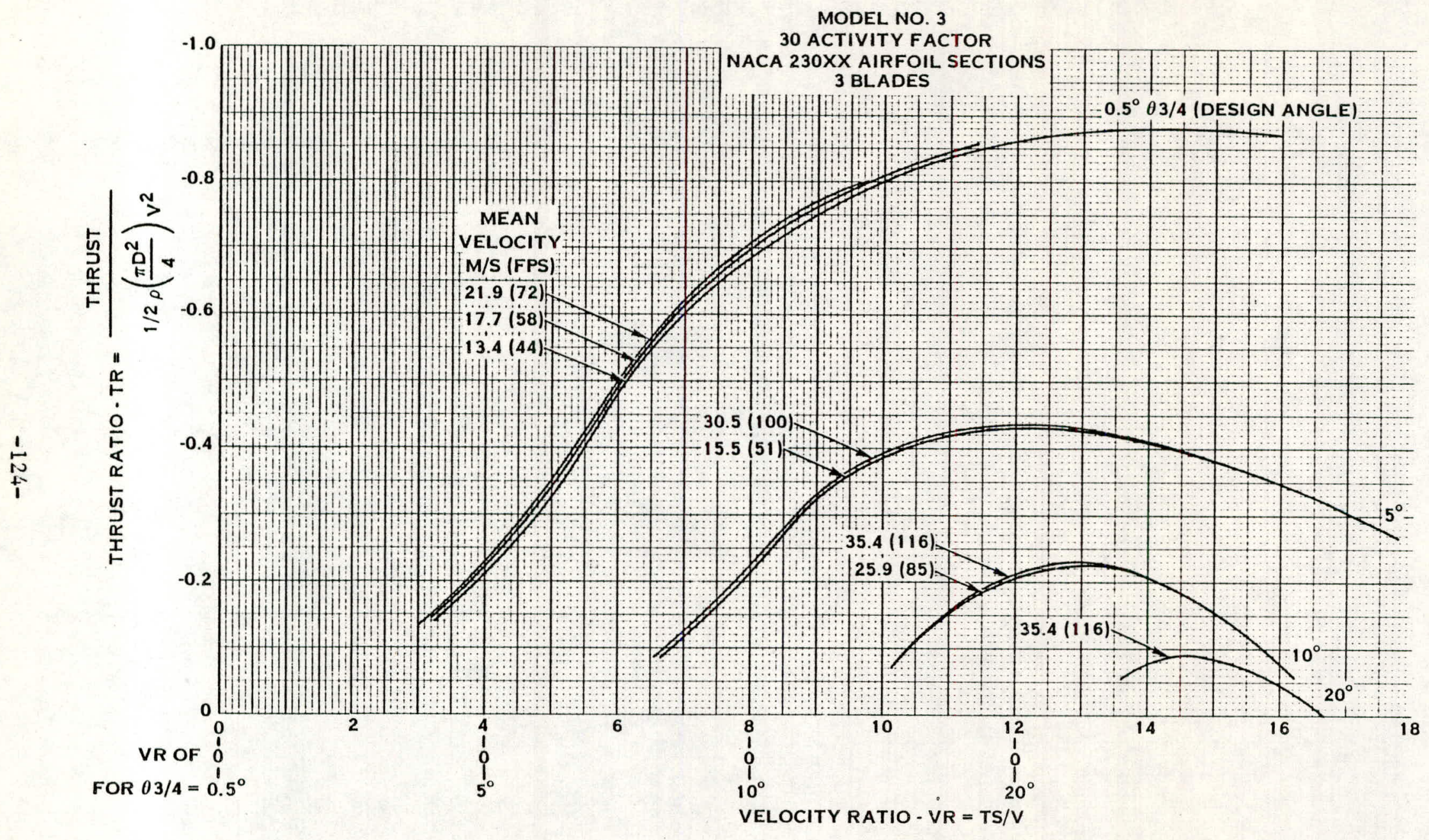

FIGURE 9-4D. EFFECT OF BLADE ANGLE AND MEAN VELOCITY VARIATION ON PREDICTED THRUST RATIO 
MODEL NO. 4

30 ACTIVITY FACTOR

NACA 230XX AIRFOIL SECTIONS

2.4 M. (8.0 FT.) DIAMETER

1 BLADE

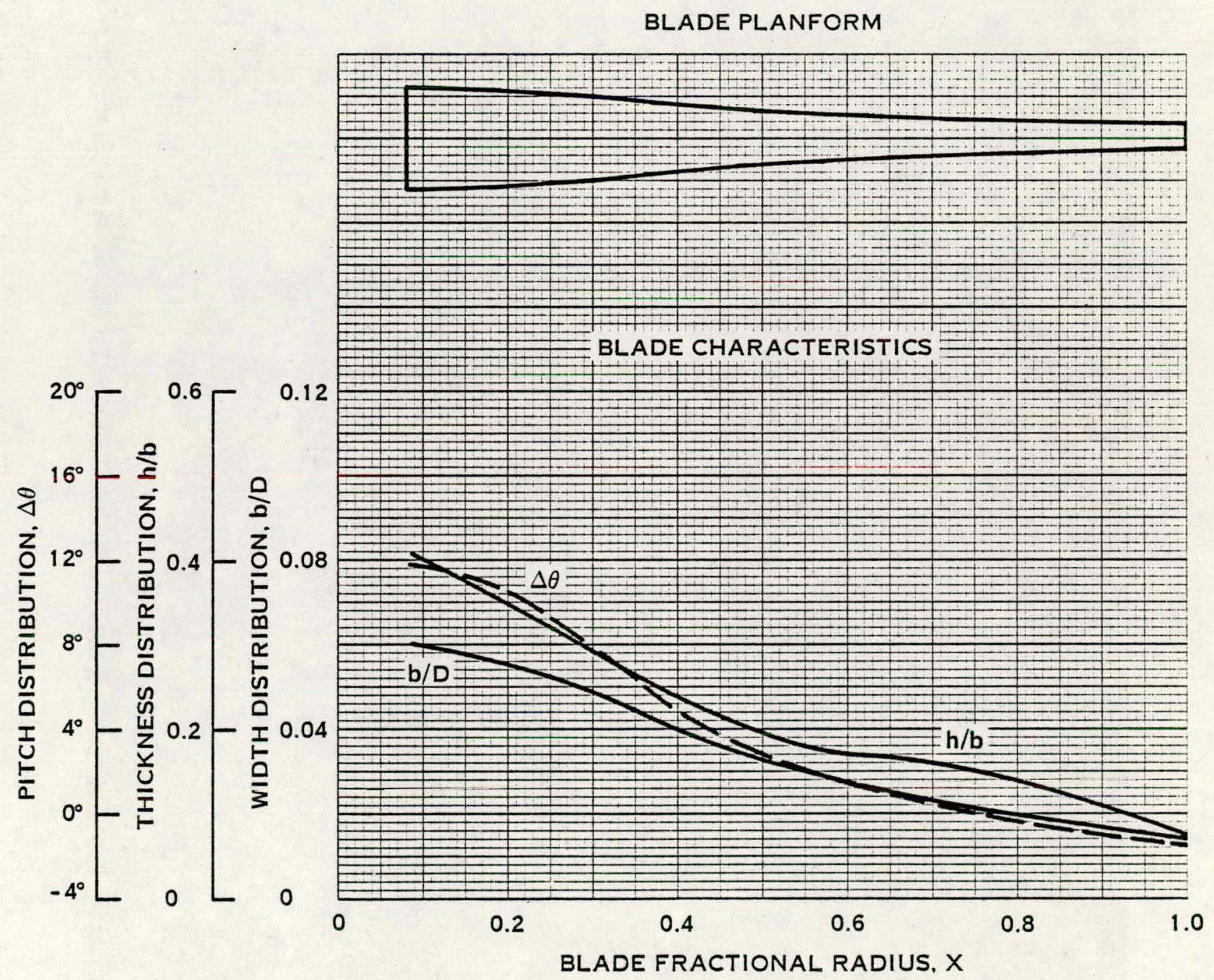

FIGURE 9-5A. BLADE CHARACTERISTICS AND PLANFORM 
MODELS NO. 2, 3, AND 4

30 ACTIVITY FACTOR

NACA $230 X X$ AIRFOIL SECTIONS

2.4M (8.0FT) DIAMETER

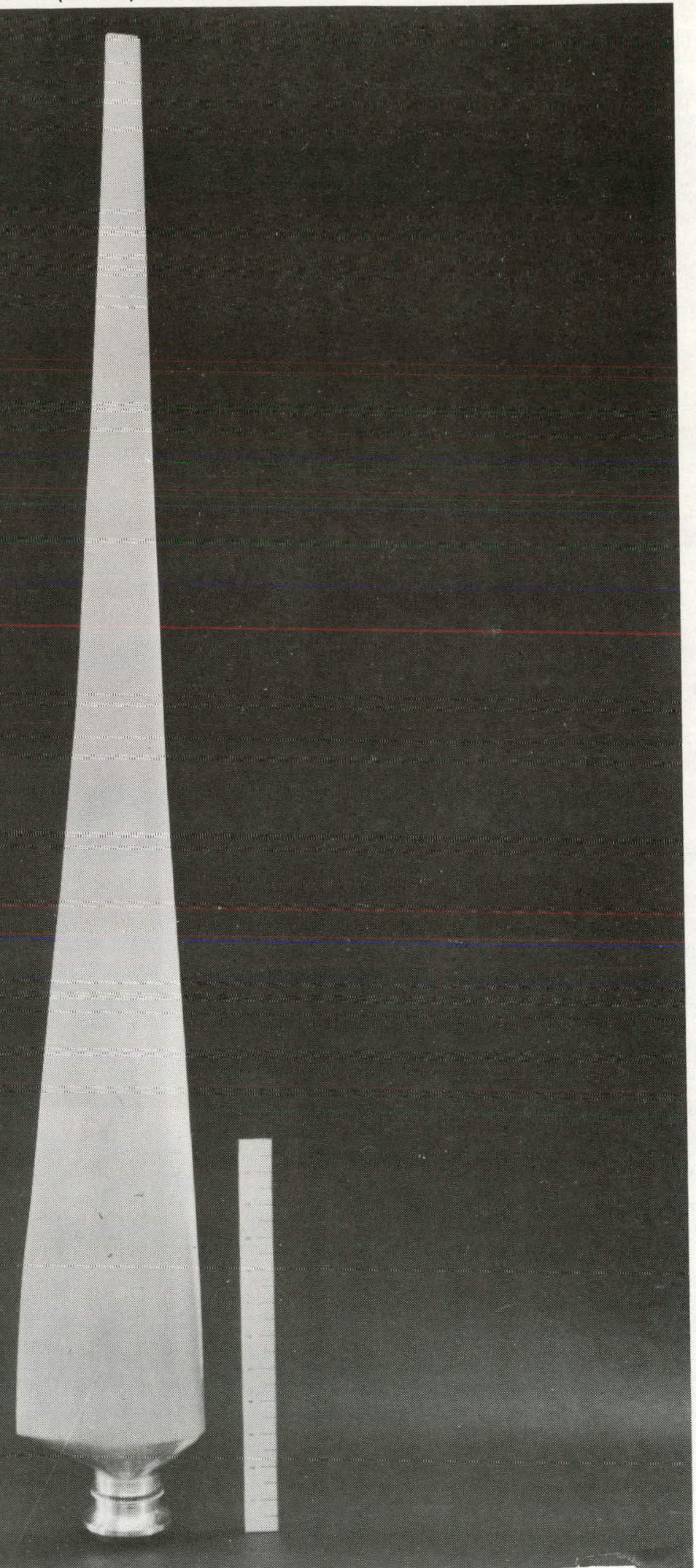

FIGURE 9-5B. BLADE SHAPE 
30 ACTIVITY FACTOR

NACA 230XX AIRFOIL SECTIONS

1 BLADE

$\theta 3 / 4=-1.5^{\circ}$

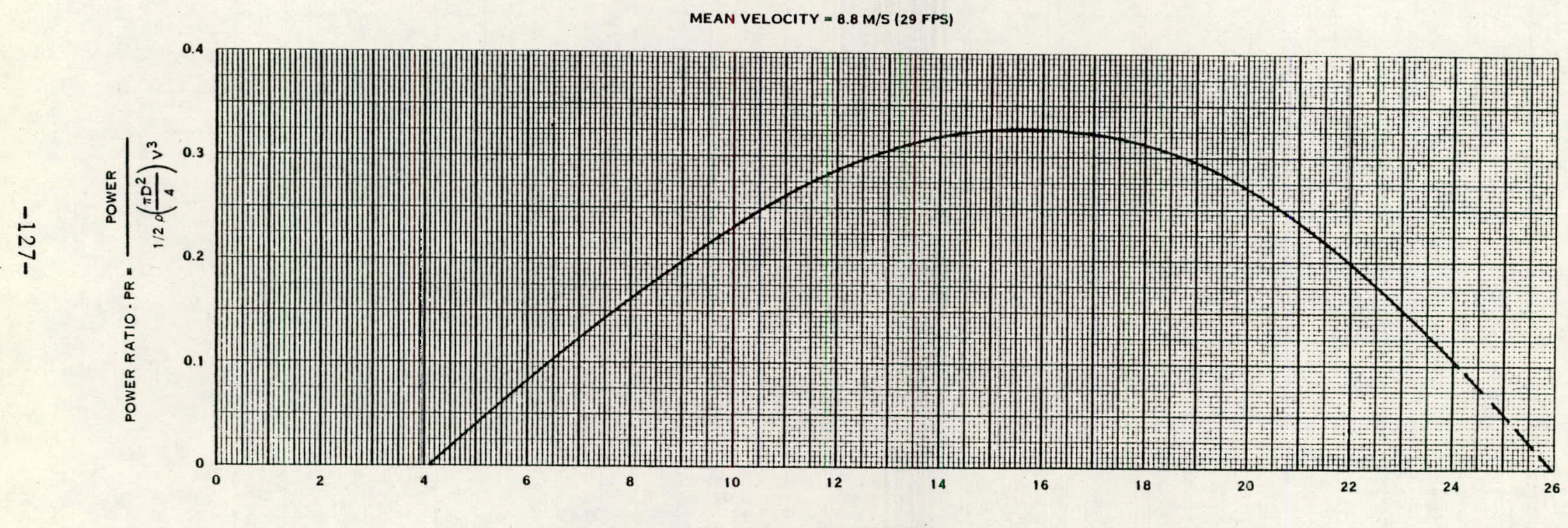

VELOCITY RATIO - VR $=$ TS $/ V$

FIGURE 9-5C. EFFECT OF BLADE ANGLE AND MEAN

VELOCITY VARIATIONS ON PREDICTED POWER RATIO 
MODEL NO. 4

30 ACTIVITY FACTOR

I BLADE

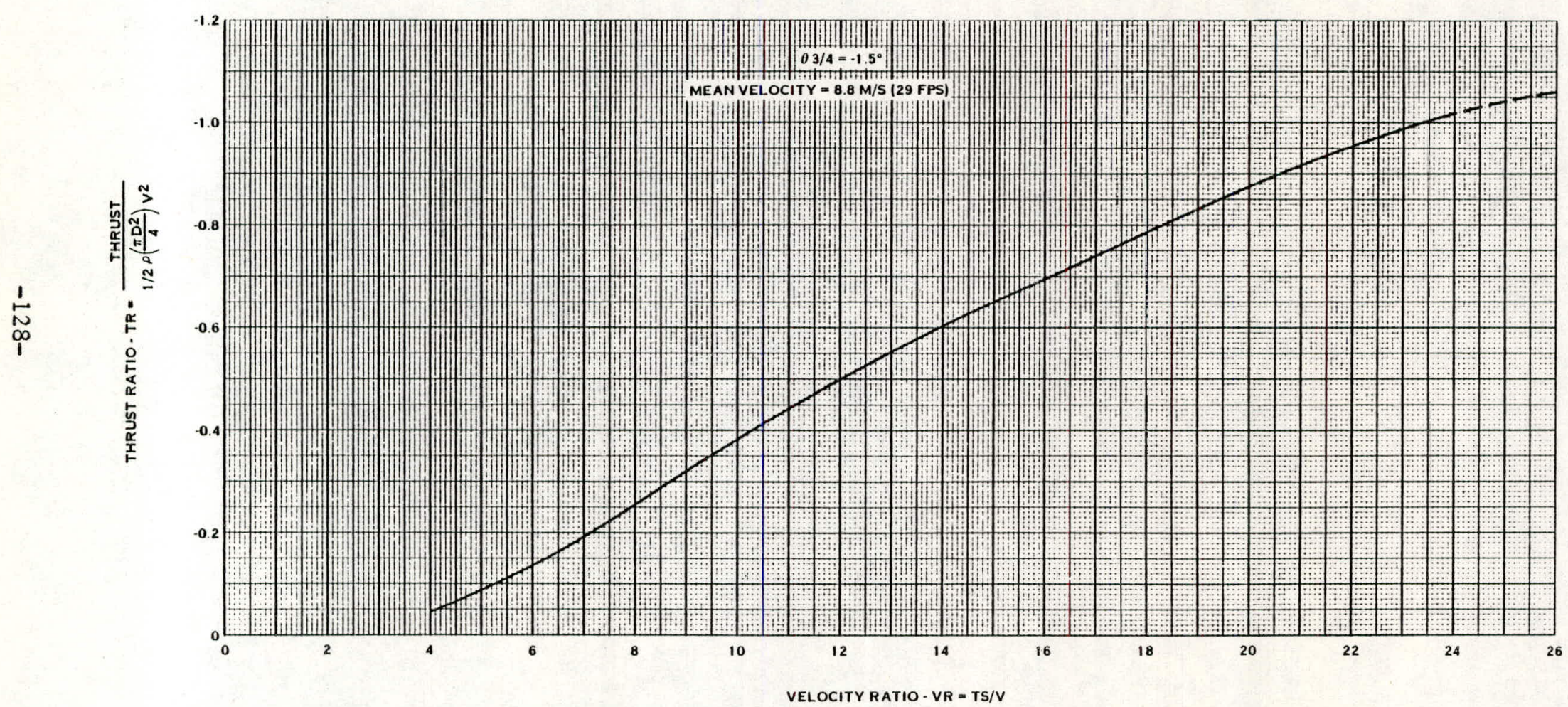

FIGURE 9-5D. EFFECT OF BLADE ANGLE AND MEAN VELOCITY VARIATION ON PREDICTED THRUST RATIO 
MODEL NO. 5

60 ACTIVITY FACTOR

NACA 230XX AIRFOIL SECTIONS

2.4 M (8.0 FT) DIAMETER

2 BLADES

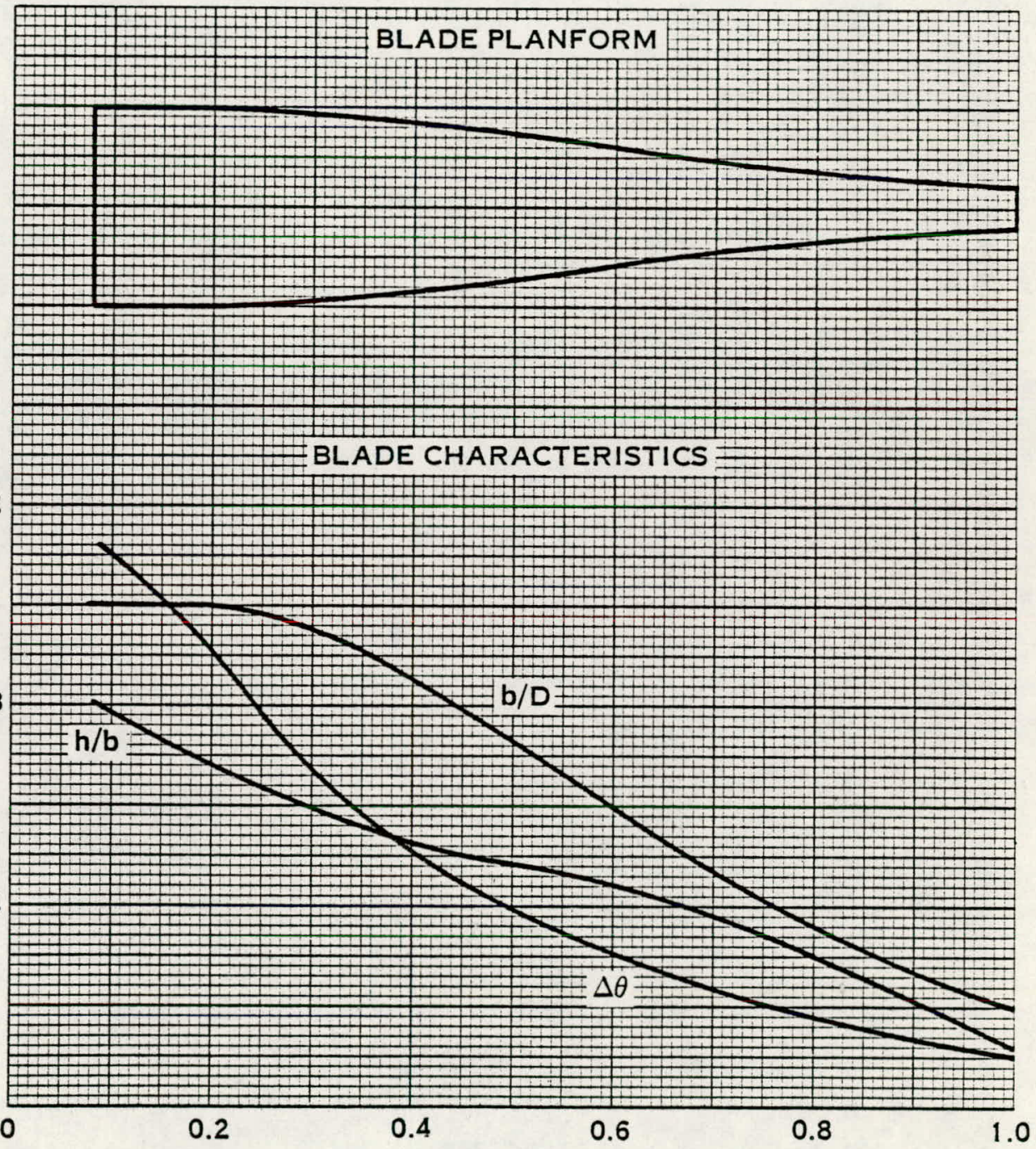

BLADE FRACTIONAL RADIUS, $X$

FIGURE 9-6A. BLADE CHARACTERISTICS AND PLANFORM 
MODEL NO. 5

60 ACTIVITY FACTOR

NACA $230 \times X$ AIRFOIL SECTIONS

2.4M (8.0FT) DIAMETER

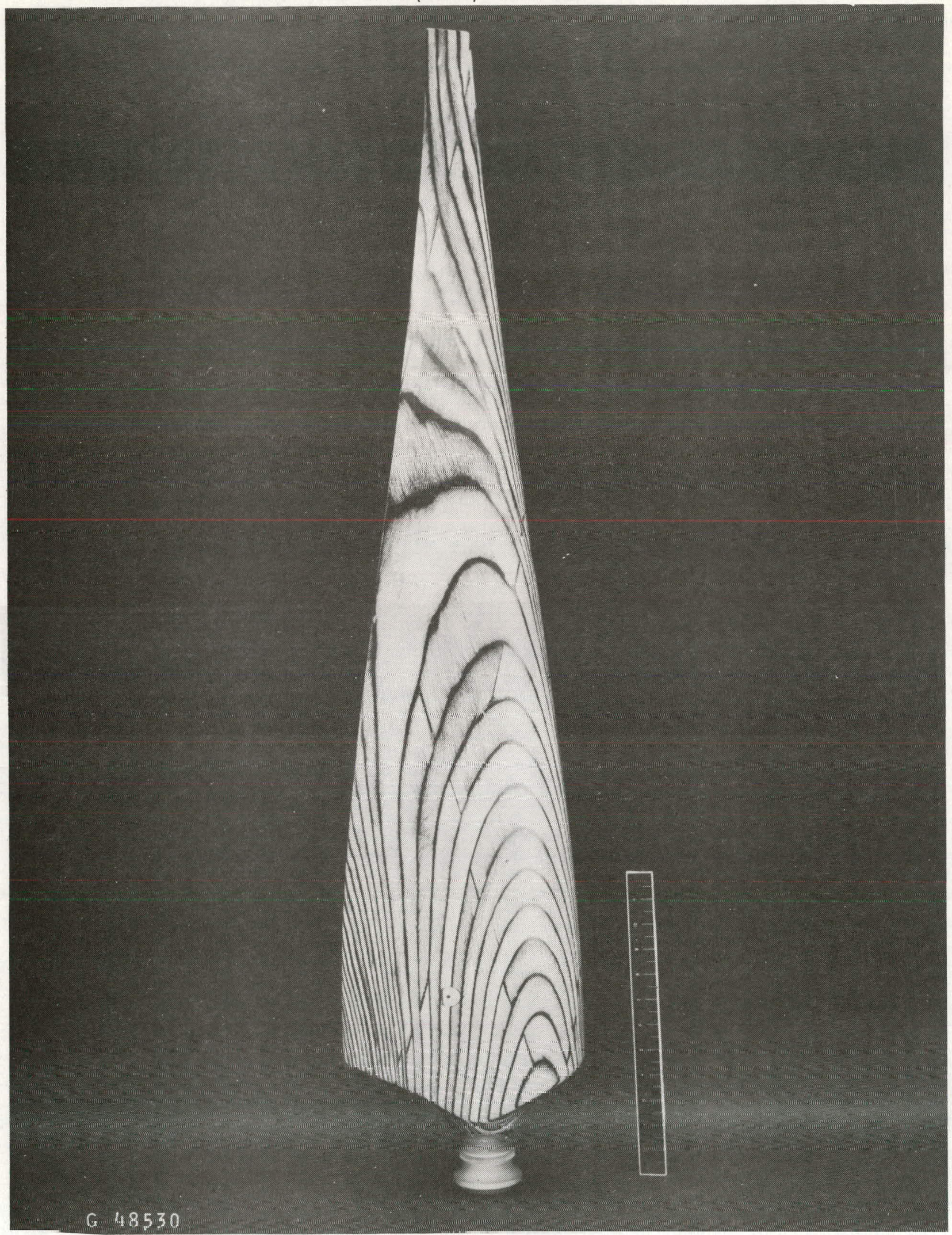

FIGURE 9-6B. BLADE SHAPE 


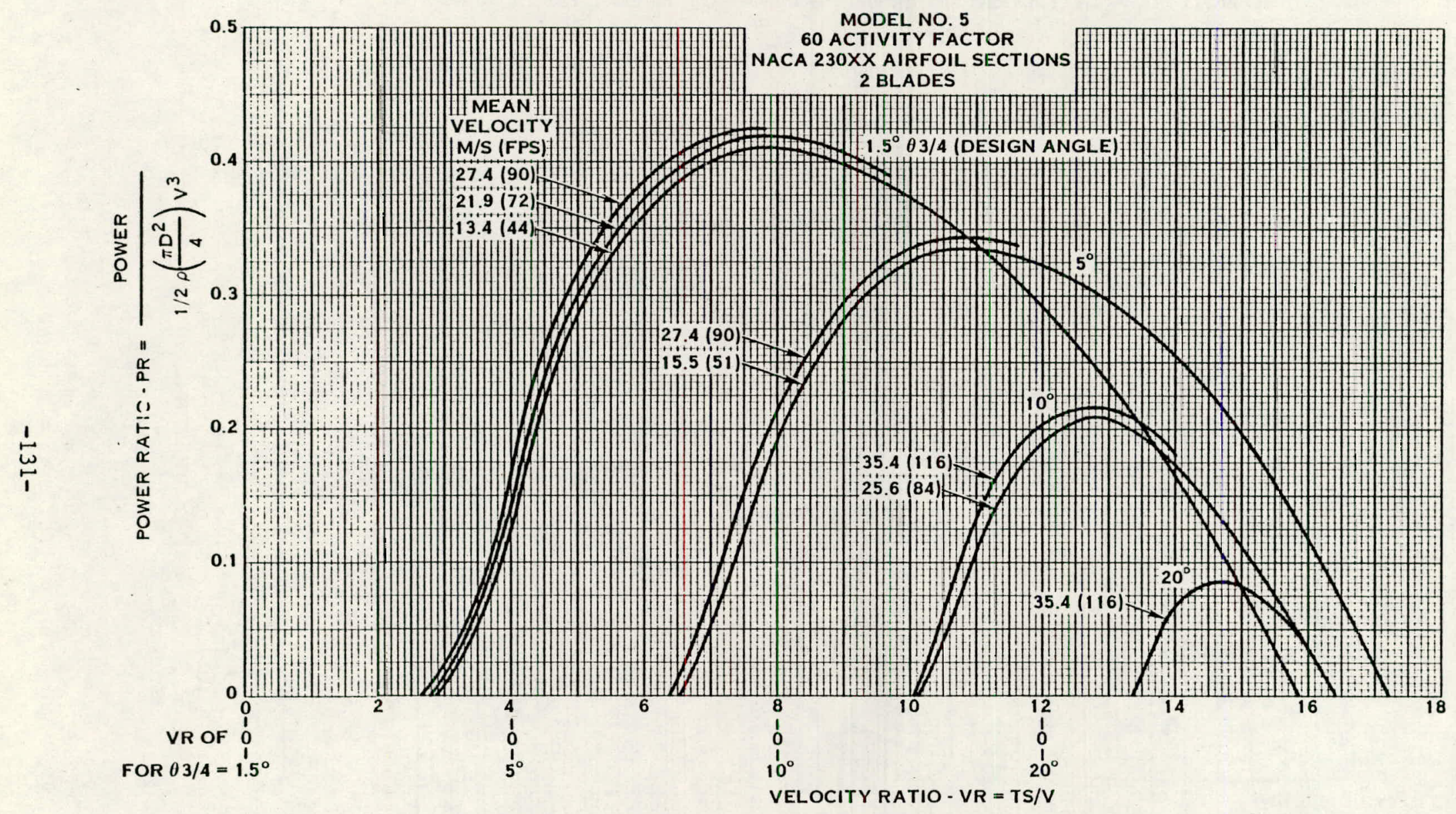

FIGURE 9-6C. EFFECT OF BLADE ANGLE AND MEAN VELOCITY VARIATION ON PREDICTED POWER RATIO 


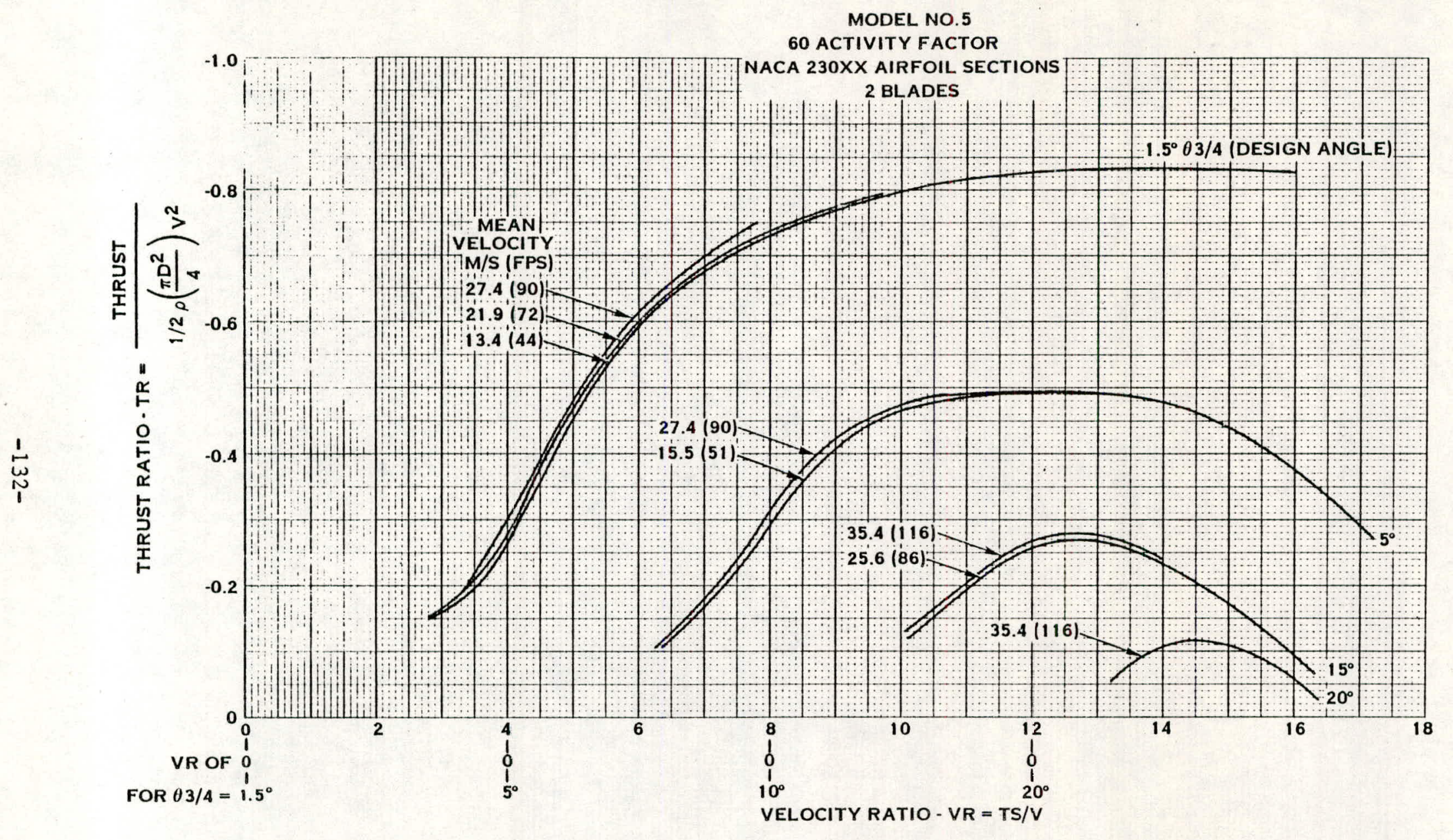

FIGURE 9-6D. EFFECT OF BLADE ANGLE AND MEAN VELOCITY VARIATION ON PREDICTED THRUST RATIO 
MODEL NO. 6

60 ACTIVITY FACTOR

NACA 44XX AIRFOIL SECTIONS

$2.4 \mathrm{M}$ (8.0 FT) DIAMETER

2 BLADES

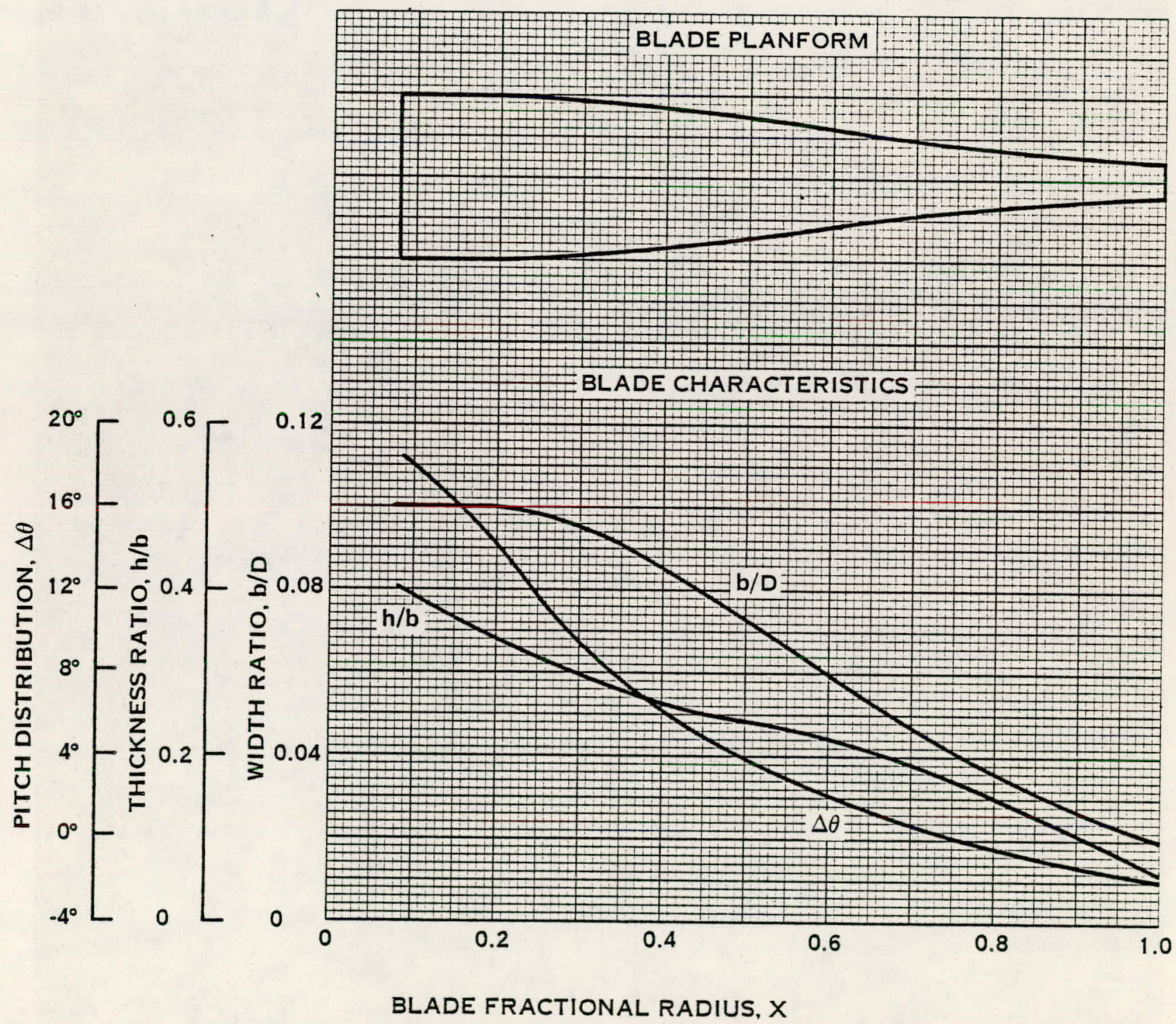

FIGURE 9-7A. BLADE CHARACTERISTICS AND PLANFORM 
MODEL NO, 6

60 ACTIVITY FACTOR

NACA $44 \times X$ AIRFOIL SECTIONS

$2.4 \mathrm{M}(8.0 \mathrm{FT})$ DIAMETER

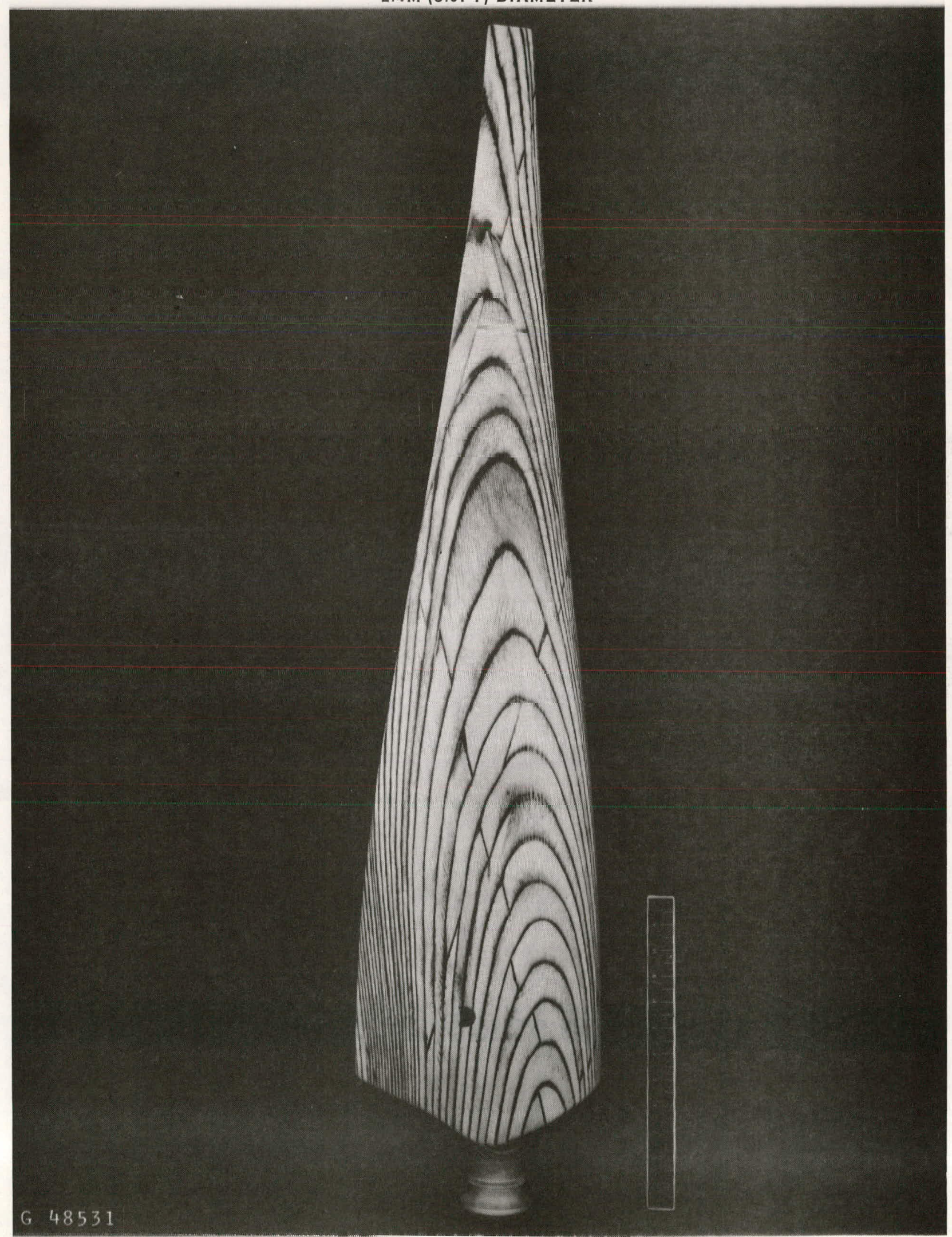

FIGURE 9-7B. BLADE SHAPE 


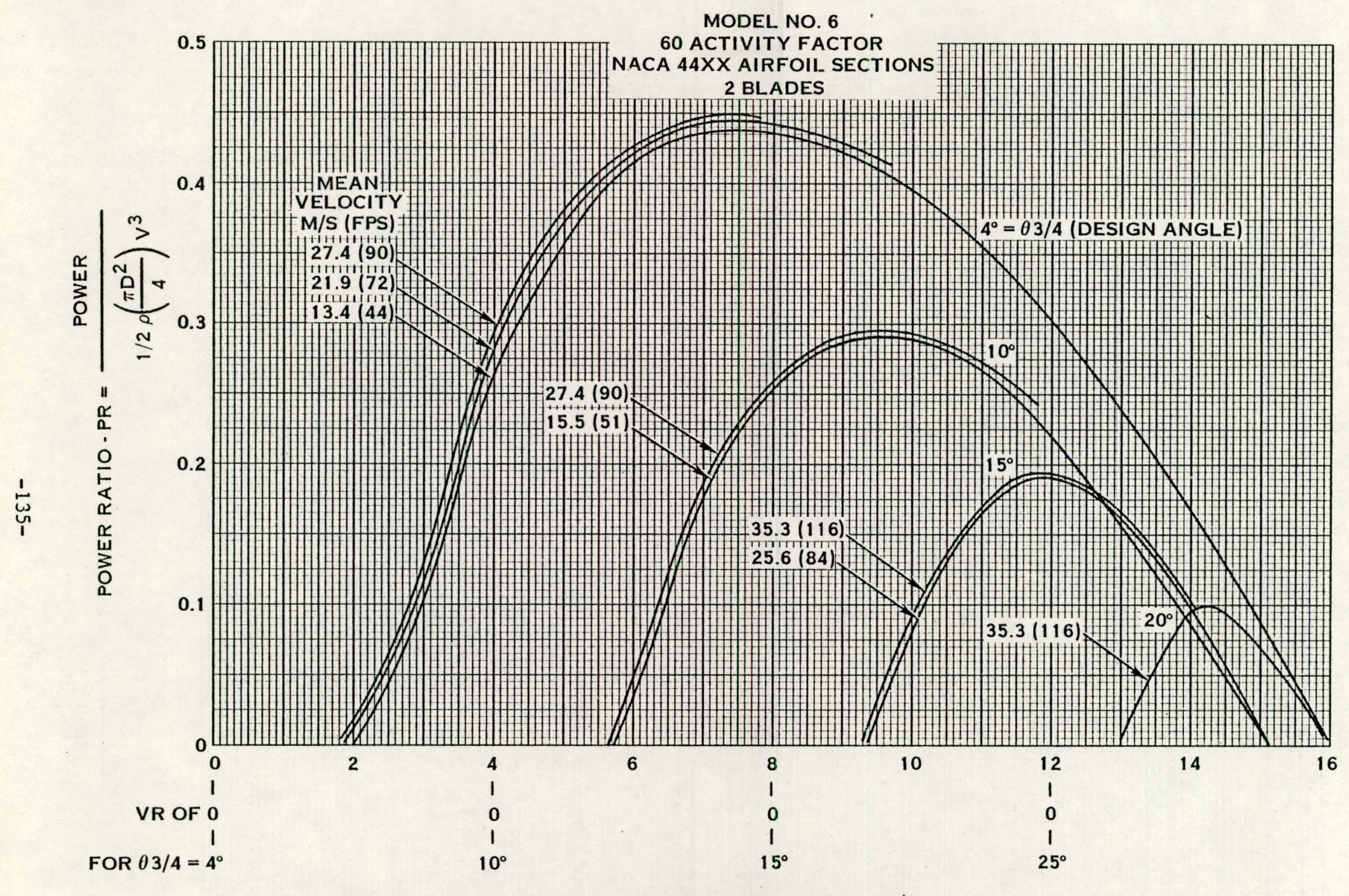

MODEL NO. 6 .

VELOCITY RATIO - VR = TS/V

FIGURE 9-7C. EFFECT OF BLADE ANGLE VARIATION AND MEAN VELOCITY ON PREDICTED POWER RATIO 
MODEL NO. 6

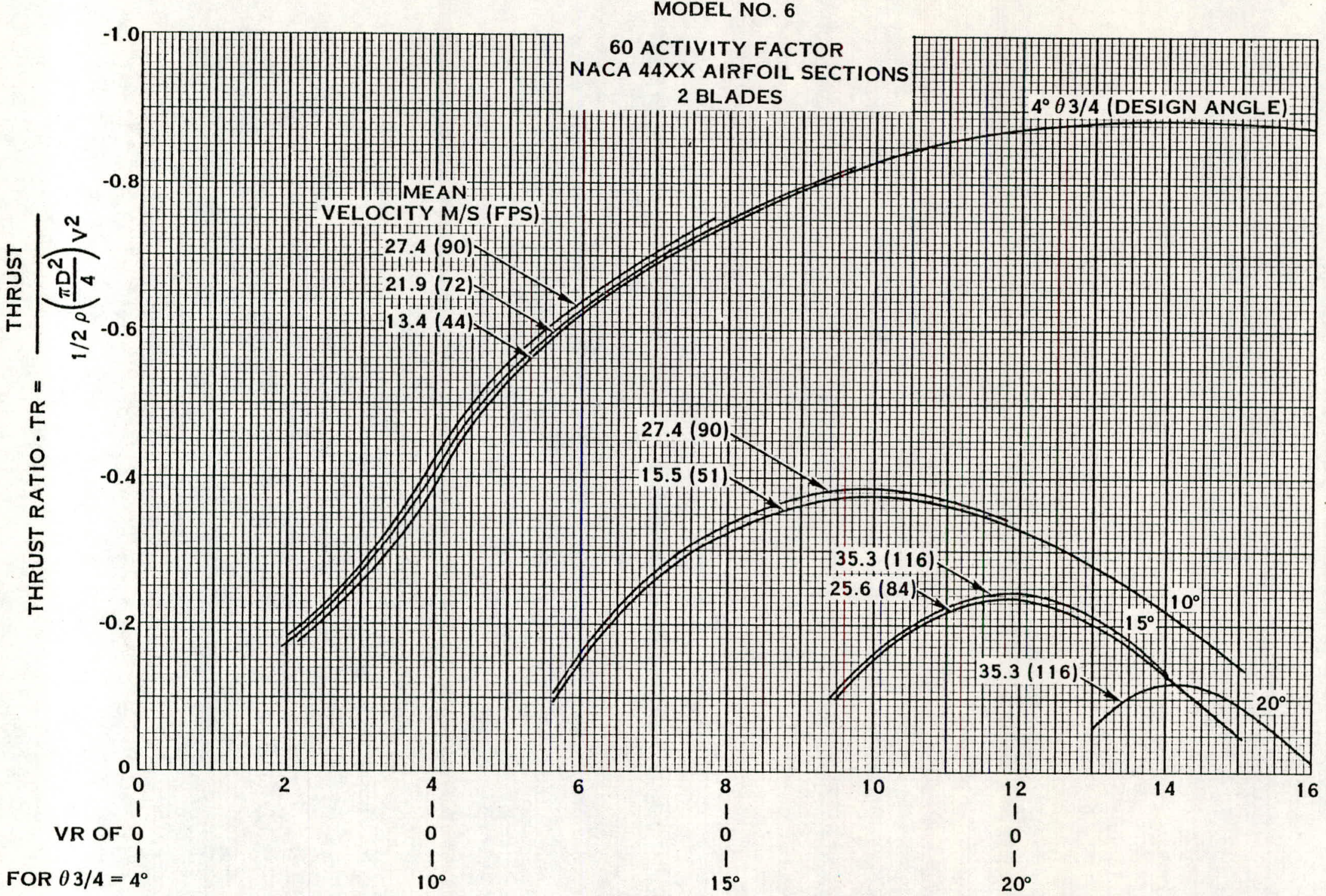

VELOCITY RATIO - VR $=$ TS $/ V$

FIGURE 9-7D. EFFECT OF BLADE ANGLE AND MEAN VELOCITY VARIATION ON PREDICTED THRUST RATIO 


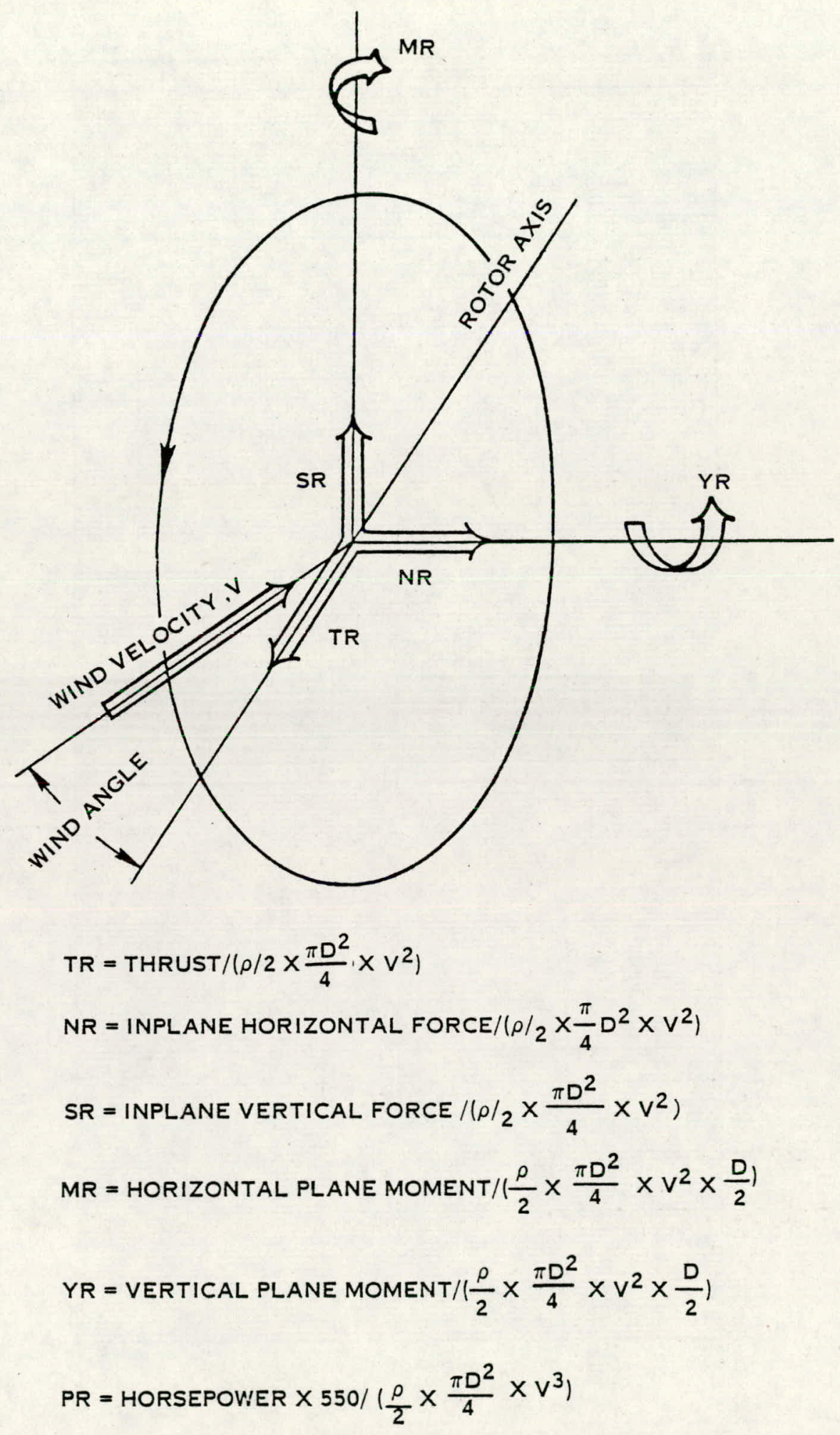

FIGURE 9-8̄A. WIND TURBINE FORCES AND MOMENTS 
MODEL NO. 2

30 ACTIVITY FACTOR

NACA 230XX AIRFOIL SECTIONS

2 BLADES

$\theta 3 / 4=-0.5^{\circ}$

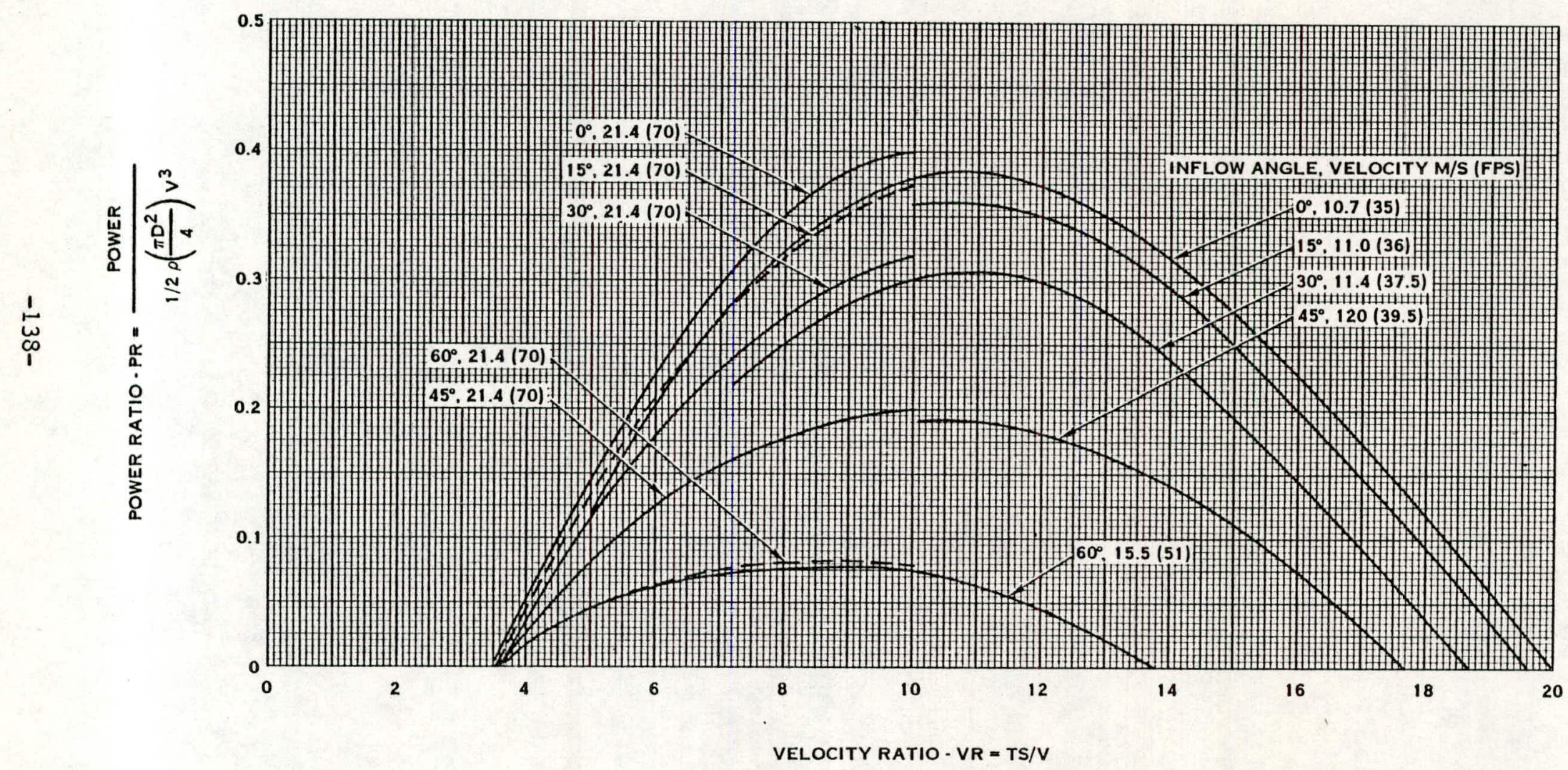

FIGURE 9-8B. EFFECT OF INFLOW ON PREDICTED POWER RATIO 


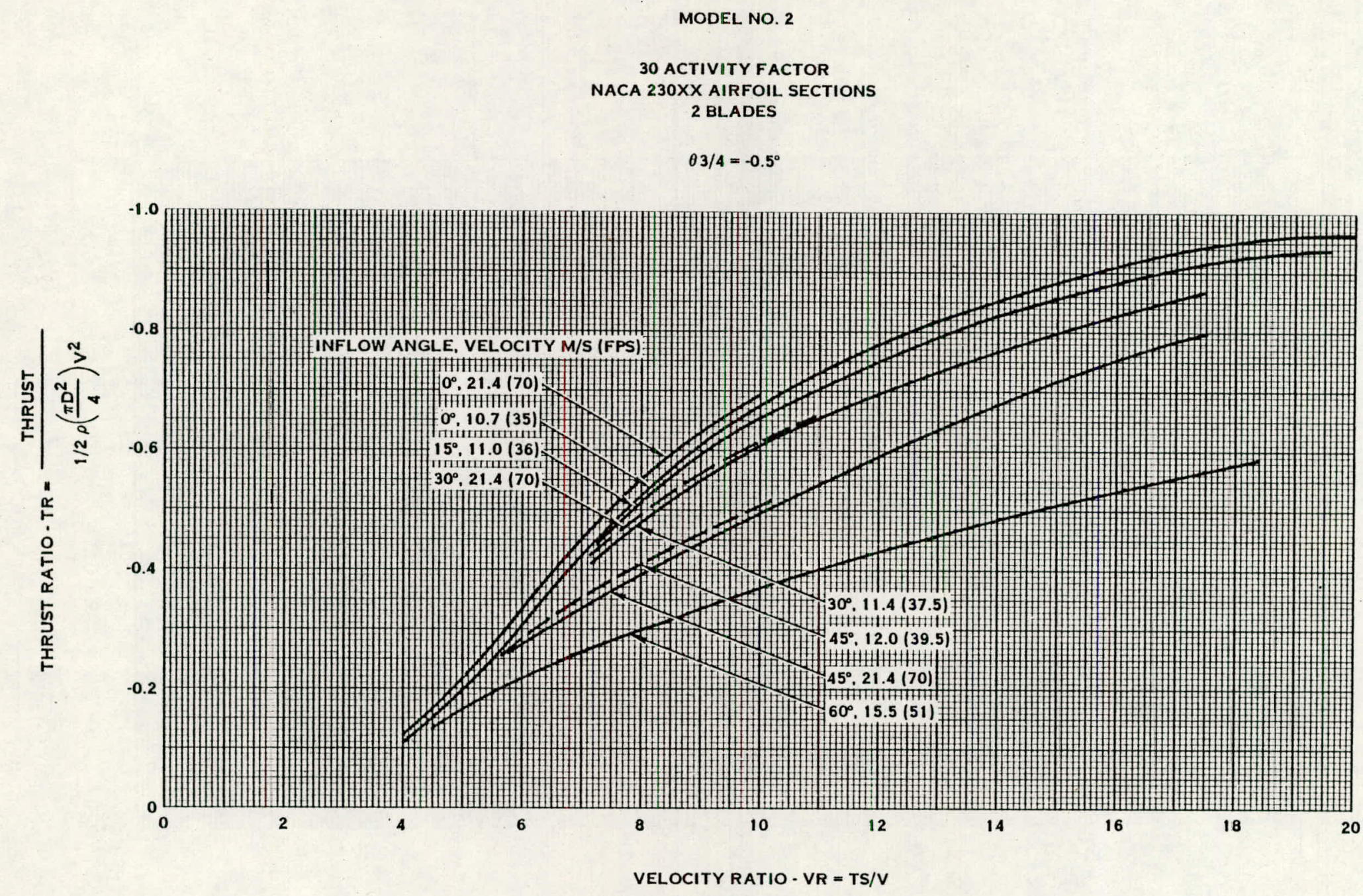

FIGURE 9-8C. EFFECT OF INFLOW ON PREDICTED THRUST RATIO 
MODEL NO. 2

30 ACTIVITY FACTOR

NACA 230XX AIRFOIL SECTIONS

2 BLADES

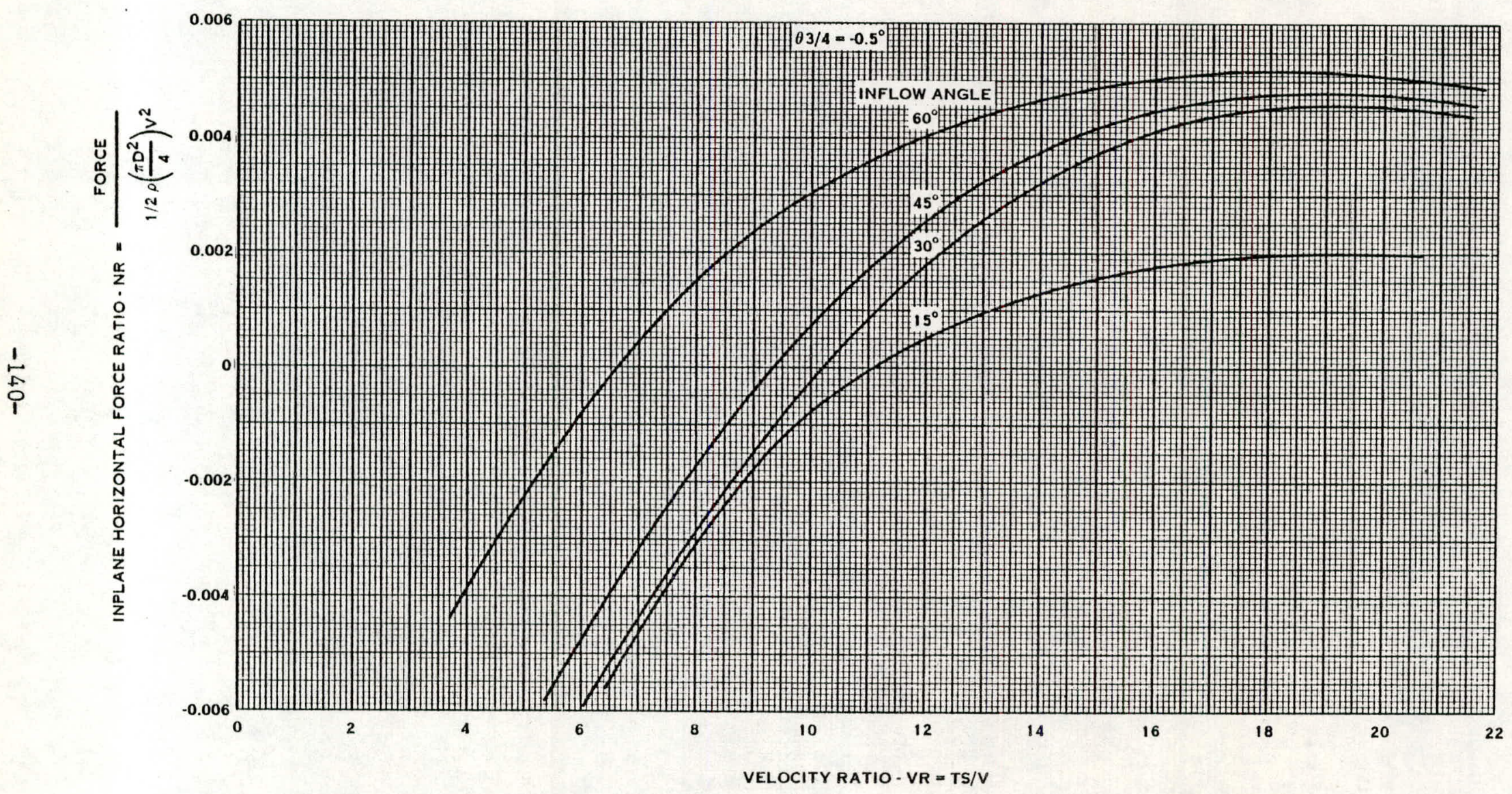

FIGURE 9-8D. EFFECT OF INFLOW ON PREDICTED INPLANE HORIZONTAL FORCE RATIO 


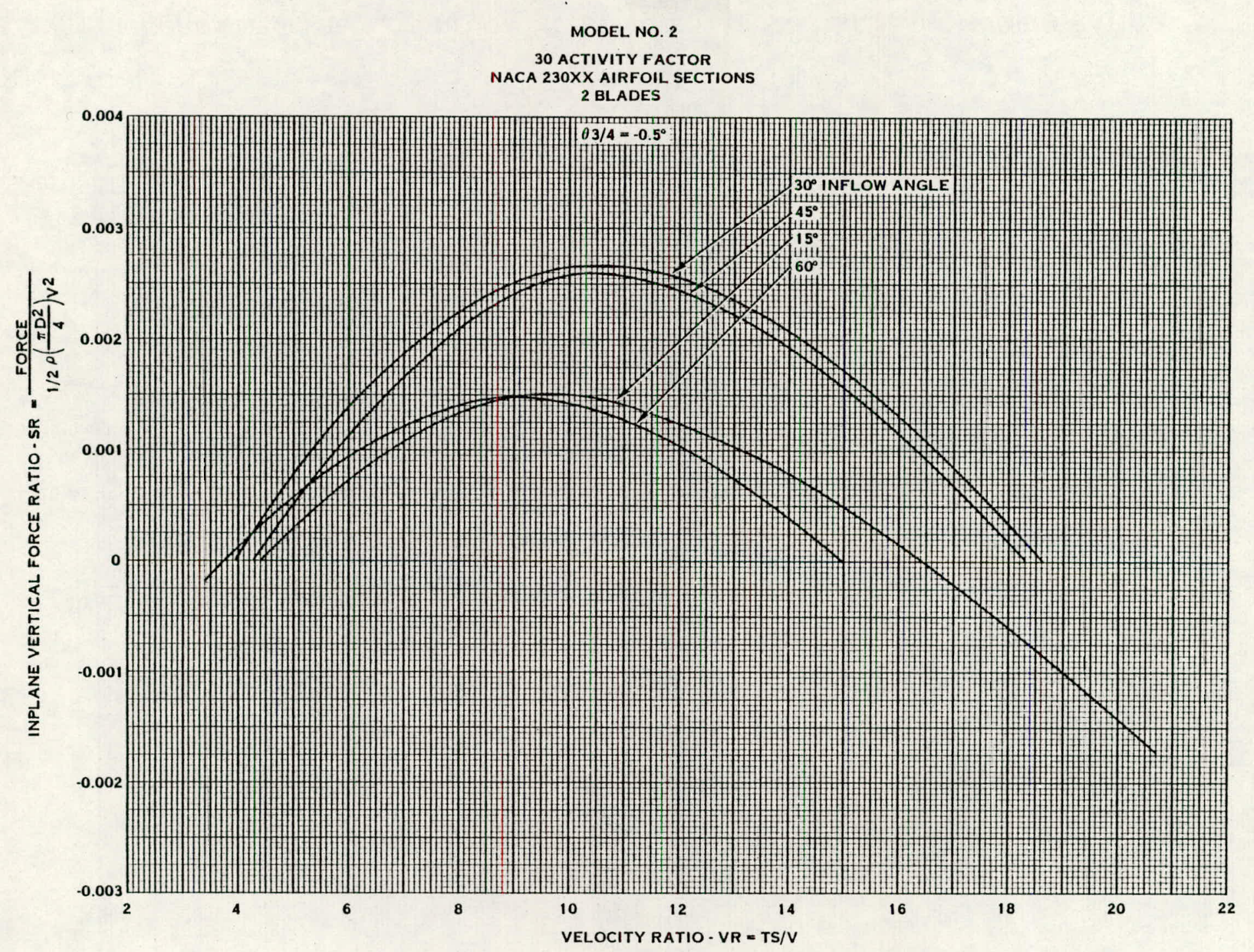

FIGURE 9-8E. EFFECT OF INFLOW ON PREDICTED INPLANE VERTICAL FORCE RATIO 
MODEL NO. 2

30 ACTIVITY FACTOR

NACA $230 X X$ AIRFOIL SECTIONS

2 BLADES

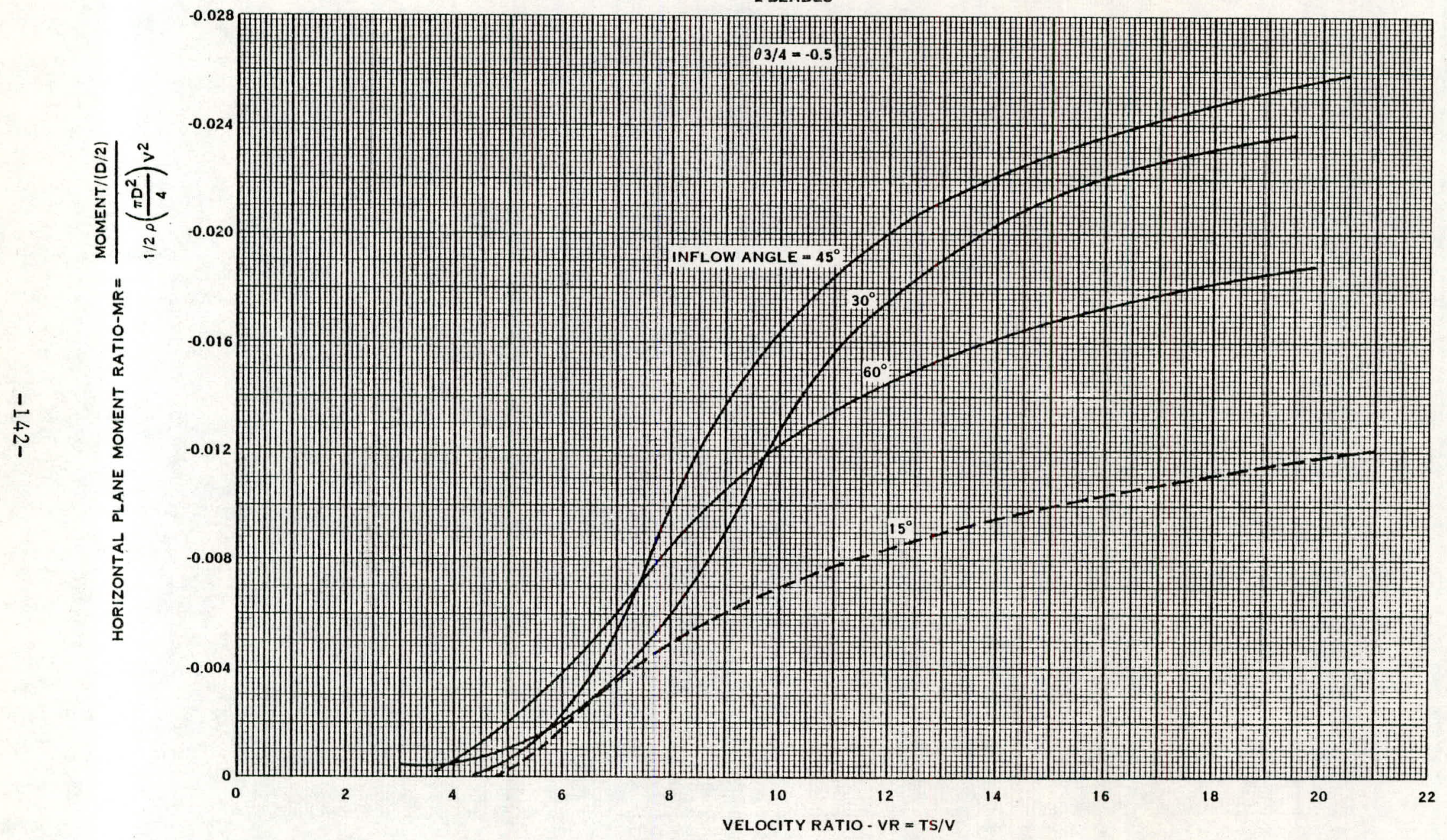

FIGURE 9-8F. EFFECT OF INFLOW ON PREDICTED HORIZONTAL PLANE MOMENT RATIO 
SO ACTIVITY FACTOR. XX AIRFOIL SECTIONS

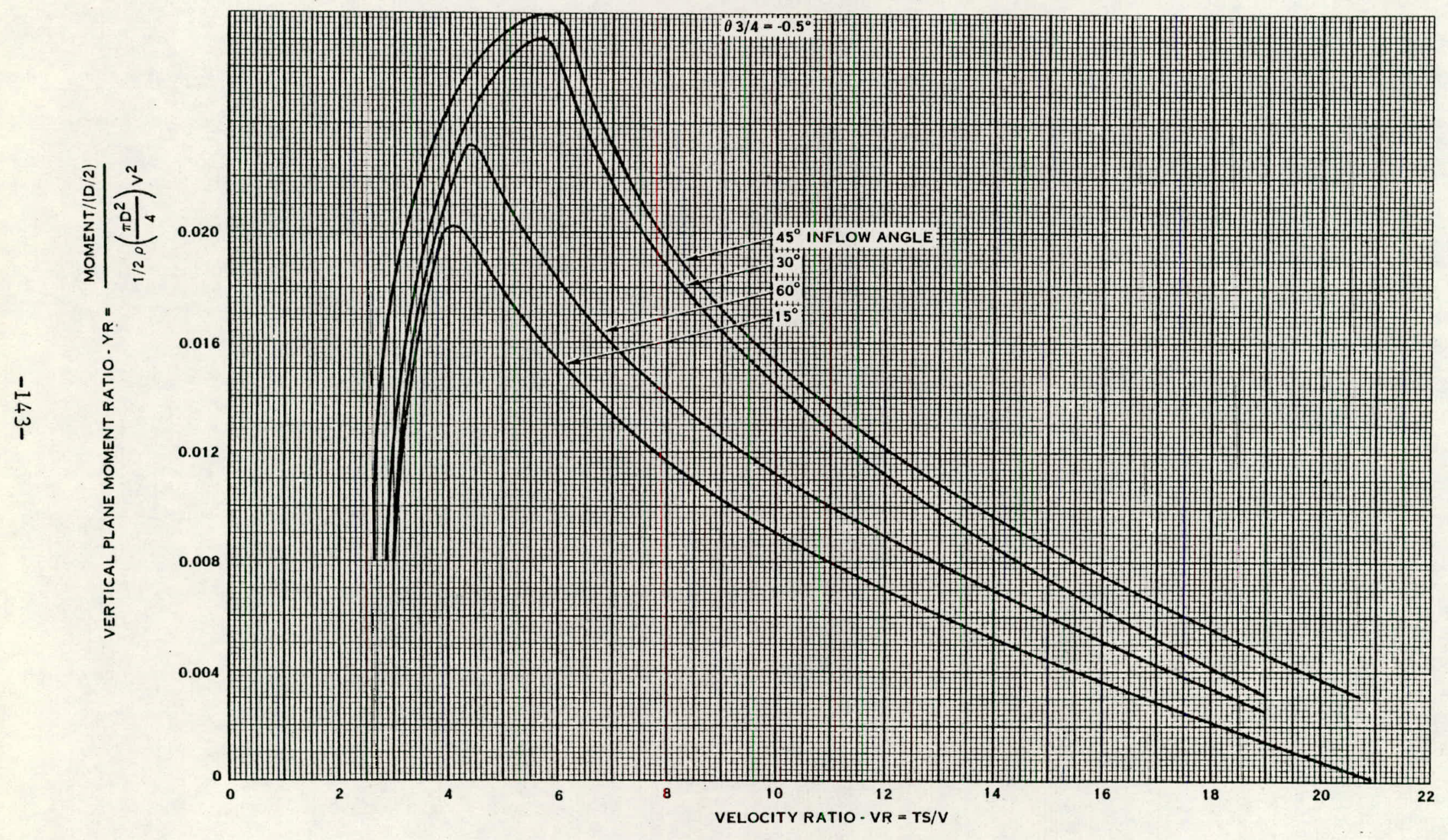

FIGURE 9-BG. EFFECT OF INFLOW ON PREDICTED VERTICAL PLANE MOMENT RATIO 
30 ACTIVITY FACTOR

NACA 230'XX AIRFOIL SECTION

2 BLADES

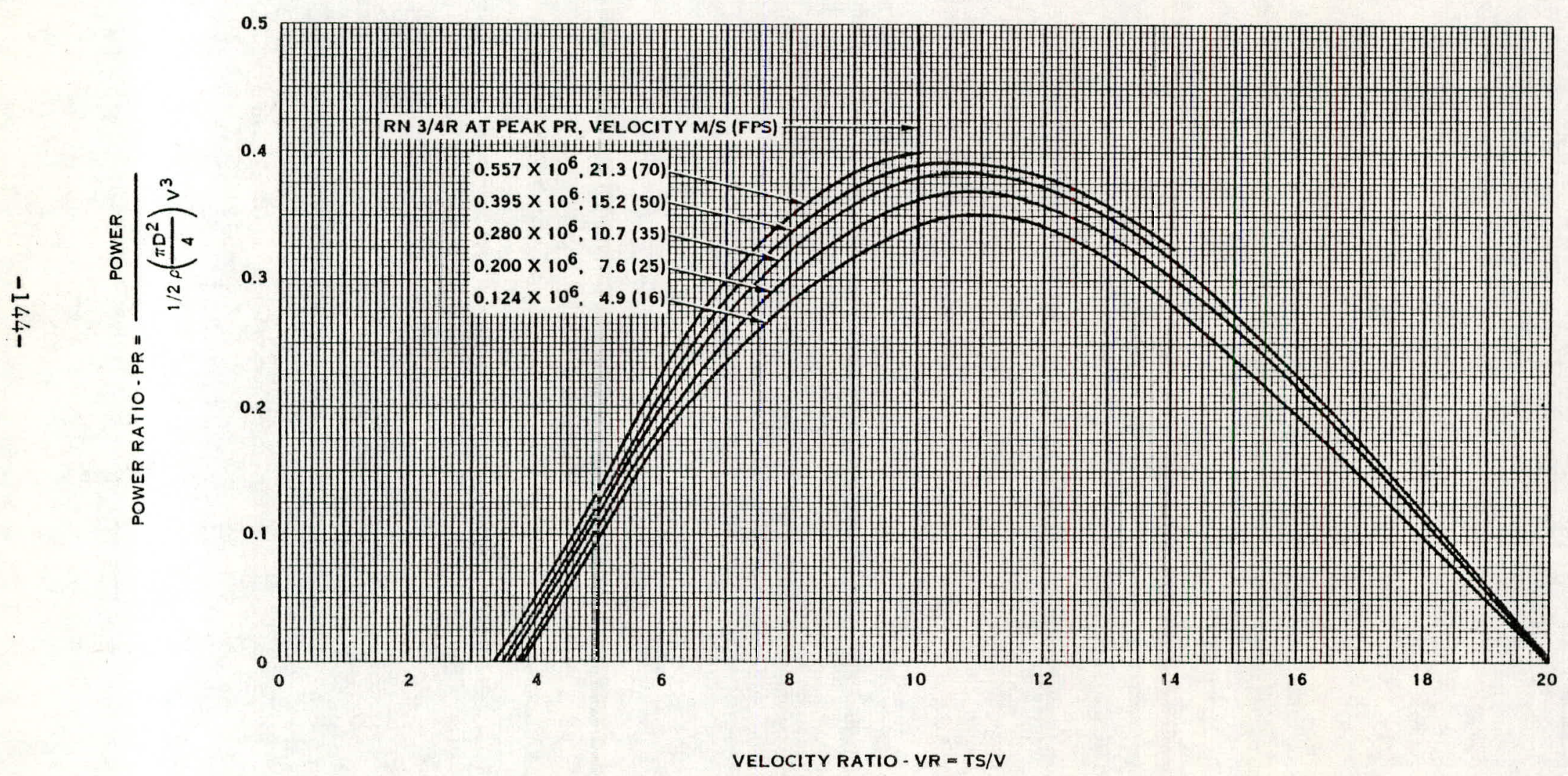

FIGURE 9-9A. EFFECT OF REYNOLDS NUMBER ON PREDICTED POWER RATIO 


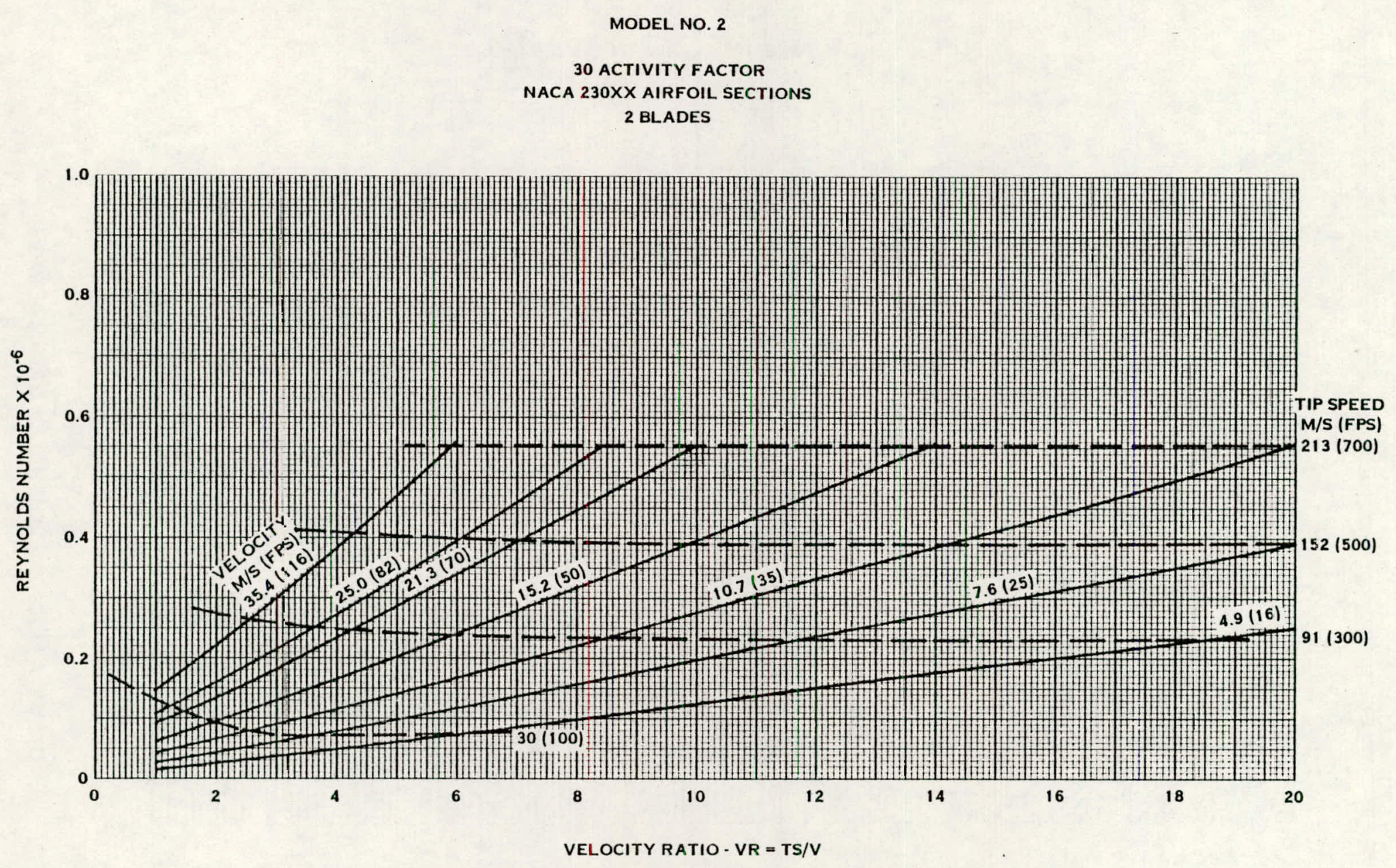

FIGURE 9-9B. REYNOLDS NUMBER NOMOGRAPH 
NACA 230XX AIRFOIL SECTIONS

2 BLADES

VELOCITY RATIO FOR PEAK POWER RATIO

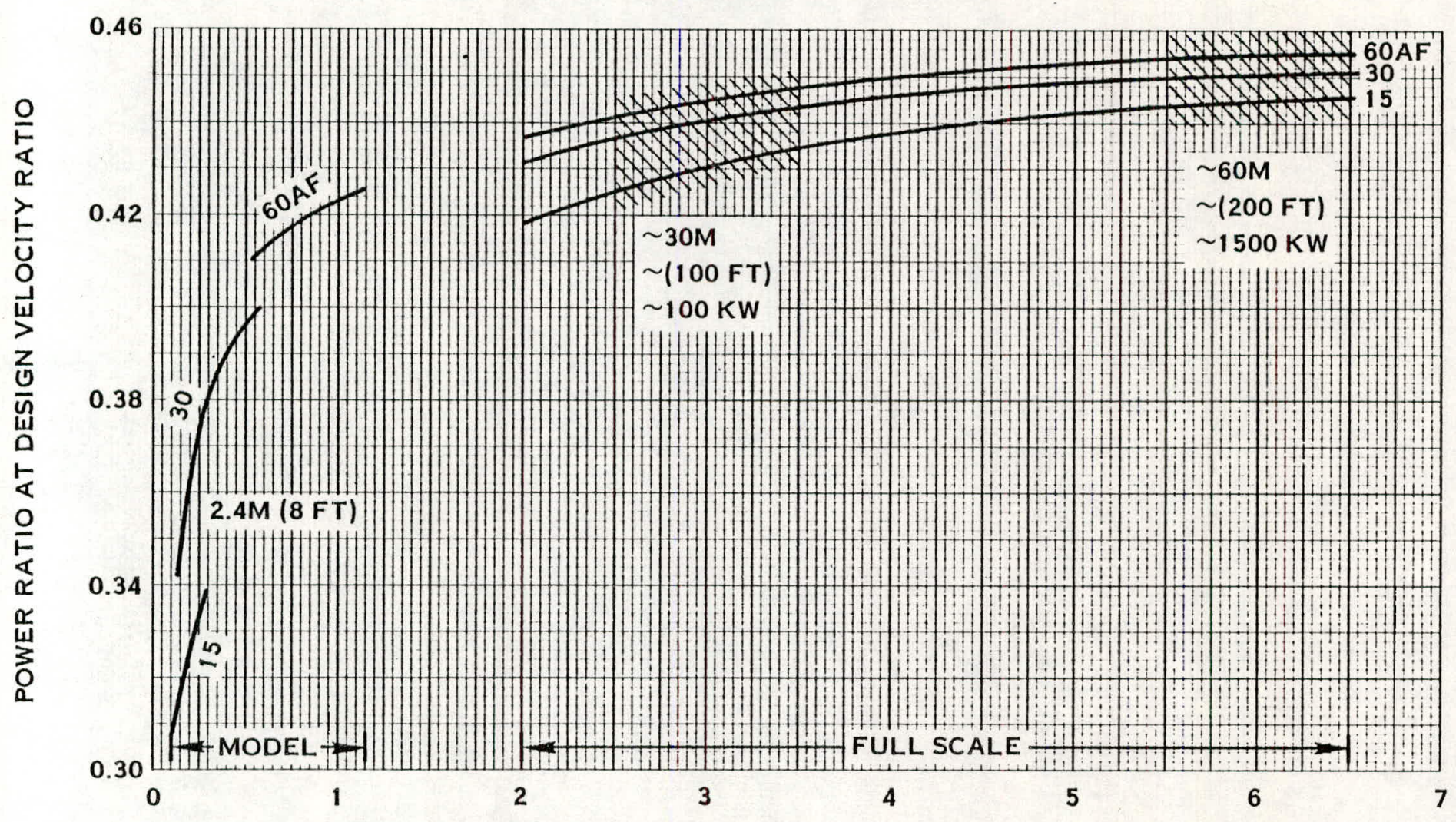

REYNOLDS NUMBER AT 3/4 RADIUS $\times 10^{-6}$

FIGURE 1. VARIATION OF POWER RATIO WITH REYNOLDS NUMBER 


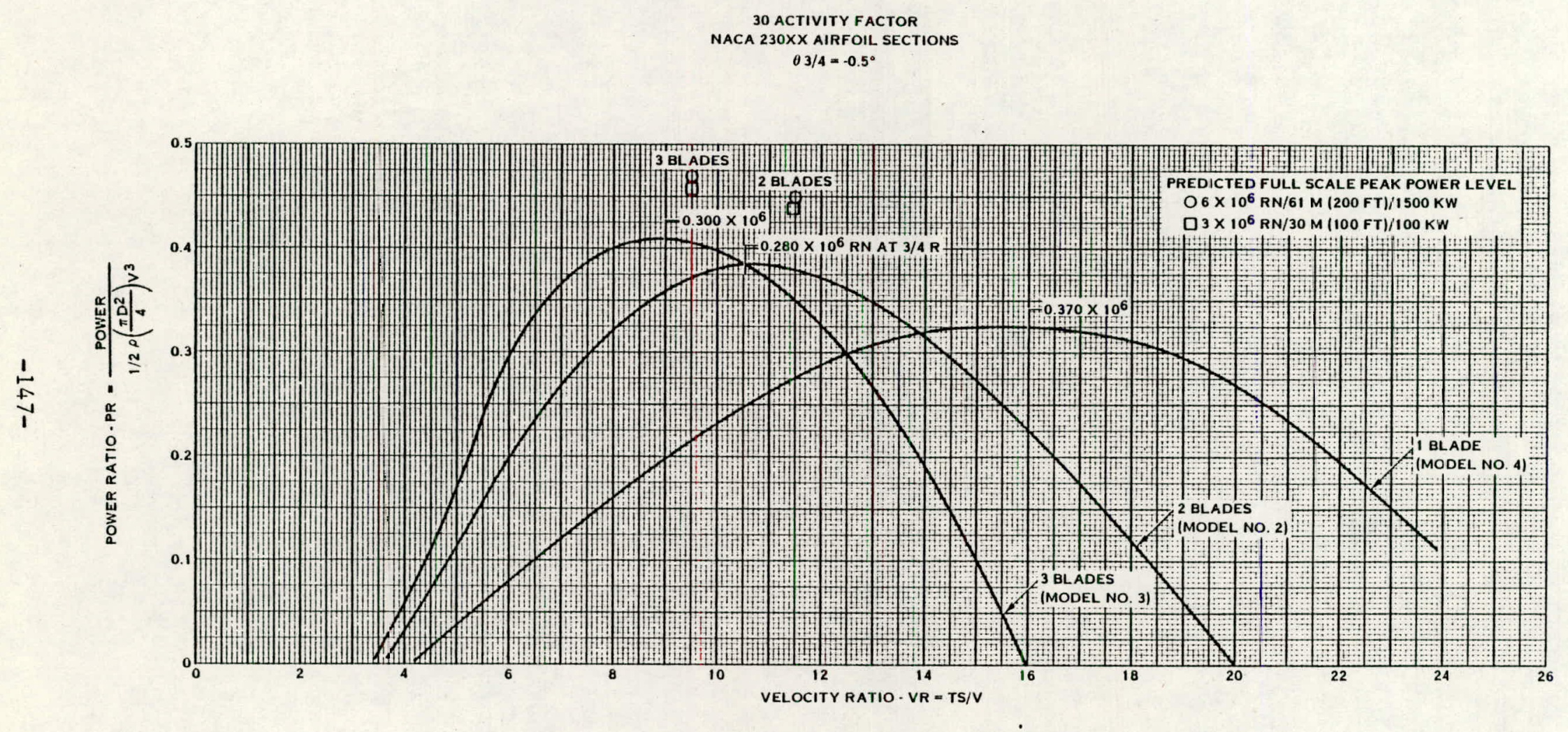

FIGURE 9-10. EFFECT OF NUMBER OF BLADES ON PREDICTED POWER RATIO 
NACA 230XX AIRFOIL. SECTIONS

AT DESIGN BLADE ANGLE

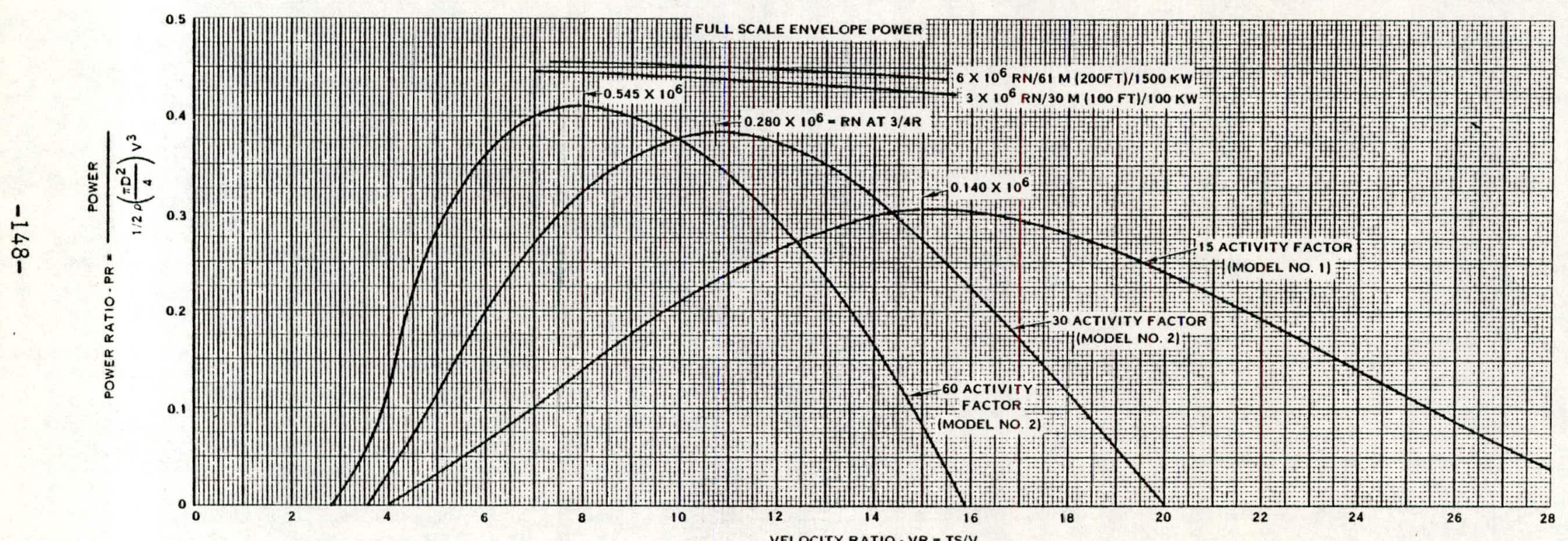

FIGURE 9-11. EFFECT OF ACTIVITY FACTOR ON PREDICTED POWER RATIO 


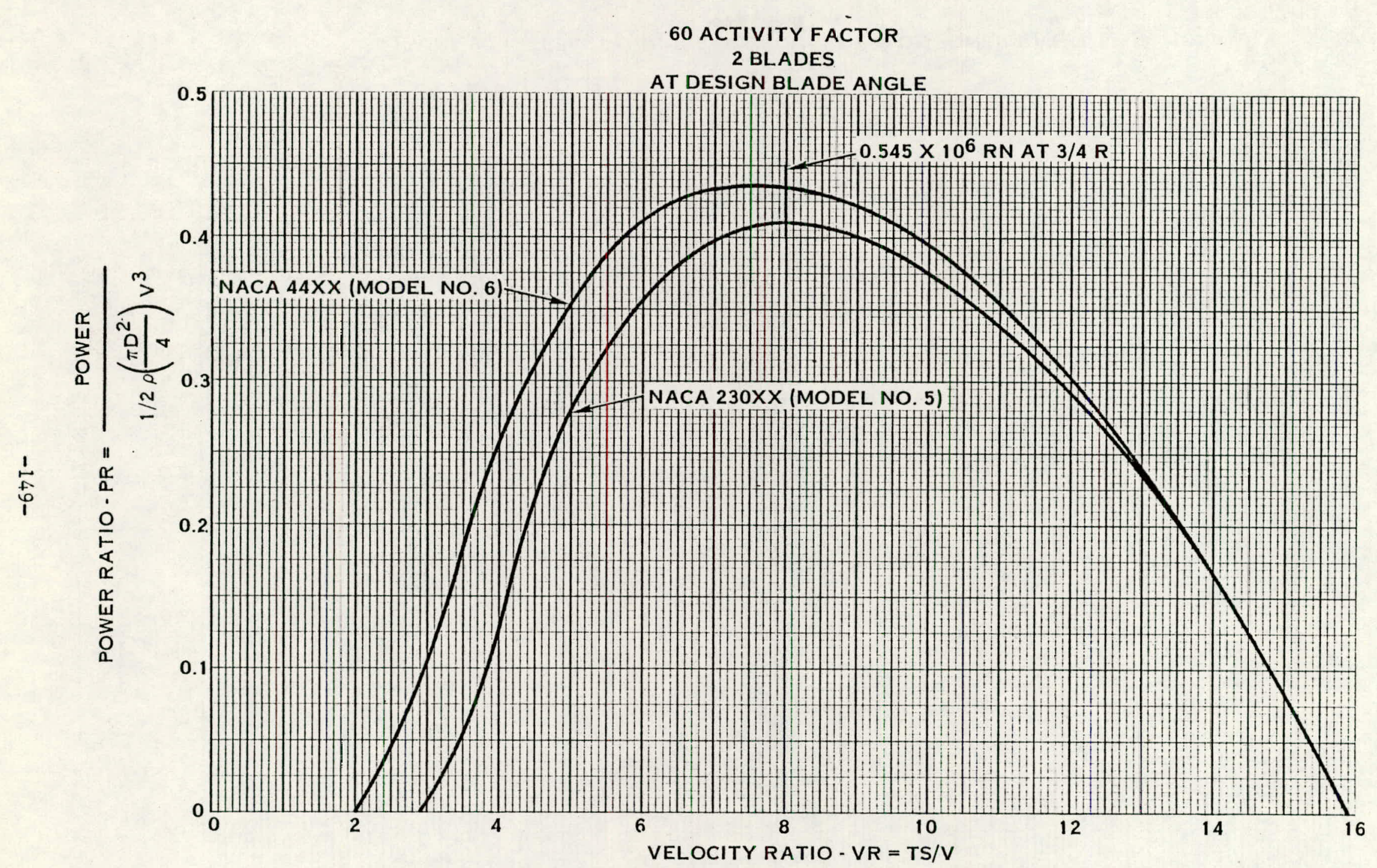

FIGURE 9-12. EFFECT OF AIRFOIL SECTION ON PREDICTED POWER RATIO 


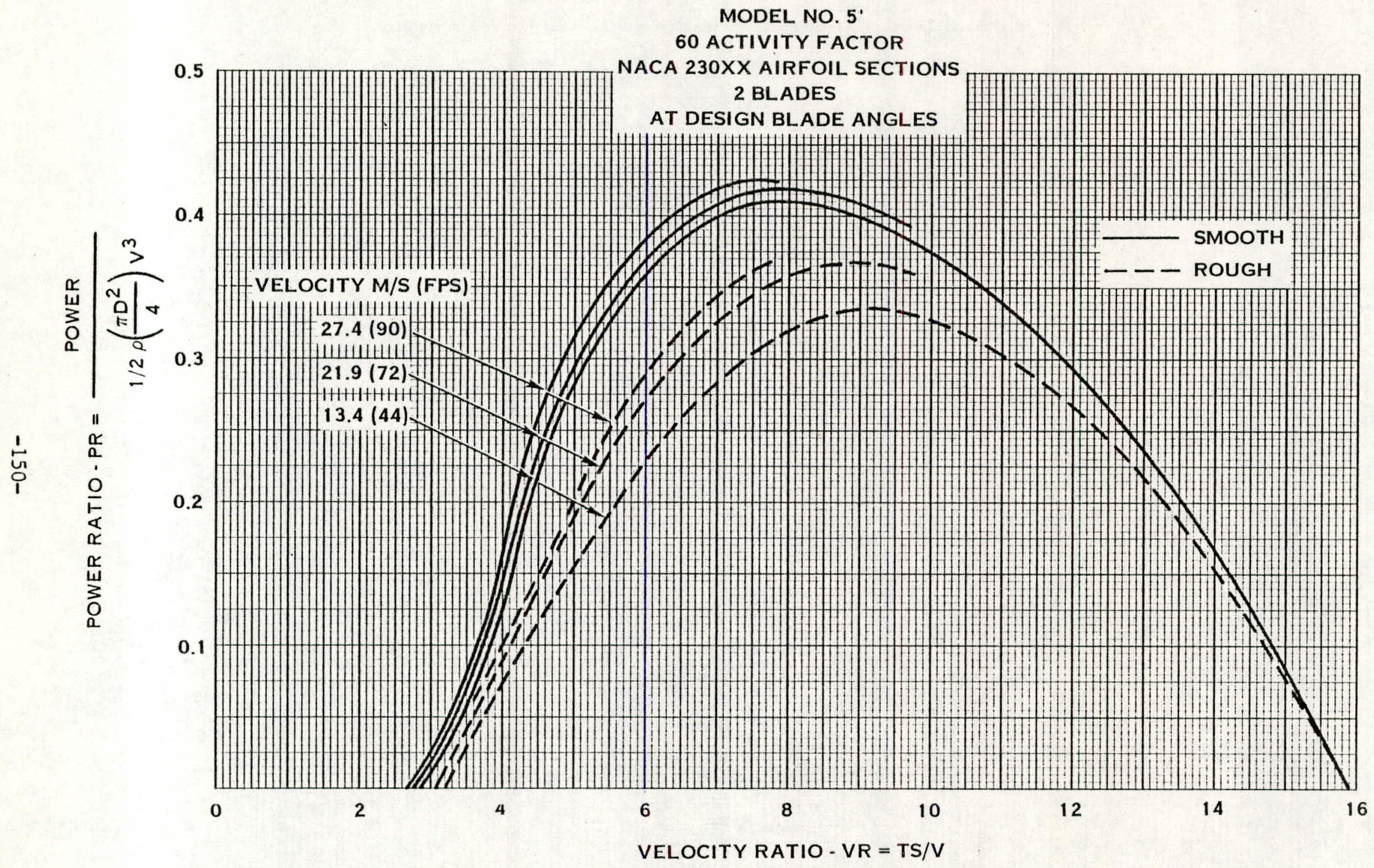

FIGURE 9-13. EFFECT OF SURFACE ROUGHNESS ON POWER RATIO 


\subsection{INTRODUCTION}

The primary objectives of this test program were to 1) establish the performance spectrum of each wind turbine model over the entire operating range of interest within structural and operational constraints, 2) to investigate the effects of geometric and aerodynamic parameters on performance and to compare to theory as discussed in Section 9, and 3) to conduct flow visualization on the wake characteristics of one model fur use in a new performance prediction method.

It was planned that the test data would cover the same range of operating conditions as included in the calculated performance presented in the previous section; thus permitting a direct comparison of test measurements and predicted performance. A complete discussion of the original wind tunnel test plan is presented in Appendix A.

The wind tunnel test program was conducted in the $5.44 \mathrm{~m}$ (18 $\mathrm{ft}$ ) throat of the United Technologies Research Center (UTRC) subsonic wind tunnel. A special test rig and tower were provided for mounting the model wind turbines. The rig incorporated a wind turbine loading system and utilized the main tunnel six component balance for measuring the forces and moments. The primary performance data were automatically recorded and reduced on line to allow continuous perusal of the results. In addition, all measurements were recorded off-line for later reduction and analysis. Unfortunately, all of the original test plan as described in Appendix A was not accomplished due to the loss of one wooden, $60 \mathrm{AF}$ blade during the initial testing of Model 5, resulting in the destruction of the test rig.

This section presents a description of the wind tunnel test facility and a discussion of the test data reduction and accuracy and of the resulting experimental model turbine performance package derived from the test data acquired prior to loss of the rig.

\subsection{WIND TUNNEL TEST FACILITY}

\subsubsection{Wind Tunnel}

The testing was conducted in the United Technologies large subsonic wind tunnel shown in figure 10-1. The tunnel is a single-return; closed throat facility with two interchangeable test sections. The tunnel main drive system consists of a $6711 \mathrm{kw}(9000 \mathrm{hp}$ ) synchronous motor and a variable-3peed cotpling driving an $8.0 \mathrm{~m}$ ( $26 \mathrm{ft.}$ ) diameter 20 bladed fan. The basic 5.48m (18 ft.) test section used in this test can attain speeds up to $89 \mathrm{~m} / \mathrm{s}(200 \mathrm{mph})$. Tunnel stagnation pressure equals atmospheric pressure, and the stagnation temperature of the airstream is held constant in the 15 to $60^{\circ} \mathrm{C}\left(60\right.$ to $140^{\circ} \mathrm{F}$ ) range by means of air exchange valves.

A 25 channel static data system is linked to the digital computers of the United Technologies Research Center to provide data reduction. On line data reduction is done with a PDP-6 computer on a terminal located in the control room. Data are also recorded on magnetic tape for more extensive off line reduction on a UNIVAC 110 computer. 
A large six-component electro-mechanical balance is located in the balance chamber beneath the test section floor. The six-component balance loads, model altitude and test parameters were displayed in the control room and automatically processed with a PDP-6 digital computer. The maximum permissible component loads on the balance are well above those developed by the models included in this program.

\subsubsection{Test Rig}

A new test rig was built for this test mainly from components already in existence in the United Technologies Research Center inventory. As concepted, the rig was constructed around existing three and fourblade propeller hubs, had an integral torque measurement system and the capability to load the wind turbine. The general arrangement of this rig is shown in figure 10-2.

The test rig consisted of the following major components:

1. A turbine drive shaft

2. A sixty toothed wheel and magnetic pickup

3. A 3.51 planetary gear-reducer

4. A $67 \mathrm{kw}$ (90 hp) Task electric motor

5. A $227 \mathrm{~kg}$ (500 lb.) Baldwin strain gage load cell for torque measurement

6. An aluminum mounting pad

7. A fairing enclosing the drive system

The Task $\equiv l e c t r i c$ motor rated at $67 \mathrm{kw}(90 \mathrm{hp})$ at $9000 \mathrm{RPM}$ is a three phase, synchronous, induction type motor with a constant torque capacity over a $9000 \mathrm{RPM}$ speed range. Since the motor speed is $a^{\prime}$ function of supply frequency and not power, at any given speed, the motor could perform as either a motor or a dynamometer depending on the loads supplied by the turbine. The planetary gear-reducer was installed on the motor to increase the peak torque potential of the system to about $305 \mathrm{~N}-\mathrm{m}$ (225.1bs.-ft.) and to increase the operational motor RPM range by a factor of 3.5 providing a higher power capacity at a given rotor speed.

The test rig was mounted on a support strut which, in turn, was attached to the turn-table of the $5.48 \mathrm{~m}$ (18 ft.) tunnel. With this arrangement, it was possible to yaw the rig up to $90^{\circ}$ while using the main tunnel six component balance to measure the forces generated by the wind turbine. An airfoil shape fairing enclosed the strut and was grounded to the tunnel's turn-table and retained by a following system which maintained the fairing's alignment with respect to the airflow while the strut yawed within. As a result, the aerodynamic tares on the strut are small and independent of the strut's yaw angle. Figure 10-3 shows a photograph of the test rig mounted in the $5.48 \mathrm{~m}\left(18-\mathrm{ft}_{\text {. }}\right)$ wind tunnel. 


\section{2:3 Test Measurements}

All of the forces generated by the wind turbine were measured on the main wind tunnel six component balance. The forces were translated from the balance resolving center to the model axes. In this manner all six components of force could be measured simultaneously.

Prior to the start of testing it was necessary to establish the aerodynamic tares on the fairing enclosing the drive system. This was done by testing at various test rig yaw angles and tunnel velocities. Lift, drag and moment tares were then developed as a function of tunnel velocity and yaw angle. These were incorporated in the data reduction system and automatically processed during the data acquisition and reduction procedure.

The primary reading used in this test from the six components measured was the drag on the balance. Once the aerodynamic tares of the fairing are removed, this is a direct measure of the wind turbine thrust. The drag or thrust loads measured were well within the capacity of the system.

Although the torque produced by the wind turbine could have been deduced from the rolling moment of the six component balance reading, a separate measurement was made of this most important parameter. By floating the entire drive/motor system the reactive torque of the turbine on the drive system could be measured through a simple load cell. This was done by attaching one end of an arm to the outer housing of the motor and by fixing the other end to ground through a Baldwin load cell. With this arrangement, the torque could he measured whether the motor was driven by or was driving the wind turbine. A special dead weight calibration of this system was performed prior to the start of the test.

Wind tunnel test conditions were set by measuring tunnel ambient conditions. Stagnation temperature and pressure were both measured in the upstream settling chamber of the tunnel circuit. The test section dynamic pressure was measured by four manifolded wall static orifices at the constant area approach of the $5.48 \mathrm{~m}$ (18 ft.) approximately $7 \mathrm{~m}(21.6 \mathrm{ft}$.$) forward of the$ rotor. This wall static pressure was applied to one side of a $703.1 \mathrm{~kg} / \mathrm{m}^{2}$ (1 psi) calibrated differential transducers while the tunnel's stagnation pressure was applied to the other side.

10.2.4 List of Instrumentation

Measurement

Turbine RPM (N)

Turbine Torque $(\gamma)$

Turbine thrust (D)

Side Force Pitch Moment Rol1 Moment

Yawing Moment
(Y)

(YM)
Lucarion

Rotor Shaft

Gearbox output

Below test section
Equipment

60 Toothed gear/magnetic pickup

Baldwin $227 \mathrm{~kg}$ (500:) 'load cell

6-component null seeking

balance 
Stagnation temperature $\left(T_{0}\right)$ Upstream settling chamber

Stagnation pressure $\left(\mathrm{P}_{\mathrm{TO}}\right)$

Static pressure (H-P)
Test section $12 \mathrm{~m}(39.4 \mathrm{ft}$. forward of rotor

Therinocouple/encoder

Kiel probe/Barometer encoder

Wall static orifices plus upstream total/ transducer

10.2.5 List of Models

\begin{tabular}{|c|c|c|c|}
\hline Activity Factor & No. of Blades & Airfoil Sections & Designation \\
\hline 15 & 2 & $230 \times x$ & Model No. \\
\hline 30 & 2 & $230 x x$ & Model No. \\
\hline 30 & 3 & $230 x x$ & Model No. \\
\hline 30 & 1 & $230 x x$ & Model No. 4 \\
\hline 60 & 2 & $230 x x$ & Model No. 5 \\
\hline 60 & 2 & $44 \mathrm{XX}$ & Model No \\
\hline
\end{tabular}

10.2.6 Test Schedule

The run schedule for the models of this program is shown in Table 10-1. A significant portion of the entire test program as defined in Appendix A was not completed due to a blade fracture early in the testing of the first $60 \mathrm{AF}$ wind turbine, Model No. 5. The blade fracture, reported in Appendix $B$ resulted in the complete destruction of the test rig and in significantly curtailing the test program. This included the curtailment of the testing of the one blade, 30 activity factor turbine, Model No. 4; the two bladed, 60 activity factor, 230XX airfoil turbine, Model No. 5; the two bladed, 60 activity factor $44 \times X$ airfoil turbine, Model No. 6 ; and the surface roughness, flow visualization and inflow testing of Model No. 2. Since this test program was conceived as an entity, the objectives could not be fulfilled. Moreover, the remaining tests are important to the aerodynamics and structural design of wind turbines and should be completed at some future time.

\subsubsection{Test Procedure}

Prior to the start of testing, tare runs were made covering the range of velocities to be investigated. The purpose of the tare runs was to eliminate the forces and moments acting on the nacelle fairing and support strut from the six-component balance readings. These tares were included in the data reduction program and were eliminated during the on-line data reduction procedure.

After the tare values had been established, performance testing of each wind turbine was initiated. The procedure was as follows:

1. A blade angle was set.

2. A tunnel velocity was set. The turbine RPM was monitored as the tunnel velocity was increased to assure that the maximun tip speed limit was not exceeded. Load was applied to reduce RPM as required.

3. The first data point was taken at the maximum RPM point. 
4. Increments of load were then applied to define the power ratio versus velocity ratio curve.

5. After all the load increments had been applied, a new velocity was set and increments of load again applied.

6. The above process was repeated until all the desired velocities at that blade angle had been covered.

7. At the conclusion of the above step, a new blade angle was set and steps one through six repeated until all desired combinations of blade angle had been tested.

\subsubsection{Test Observation}

In addition to the recorded data, several interesting observations were made during the course of the testing pertaining to model behavior and data quality. These observations are presented herein to provide the reader with additional insight regarding the operating characteristics of the model wind turbines and in assessing the quality of the measured data.

\section{A. General Observations}

Testing of model wind turbines can be accomplished with good results if care is taken to obtain highly accurate velocity maasurements. In addition, since Power Ratio (PR) is the prime measurement, particular care should be taken to assure that the wind turbine torque measurement is accurate and repeatable. It is also desirable to utilize models as large as feasible and have blade flow transition fixed near the leading edge to minimize Reynolds number effects. Specific observations are presented below for test models.

\section{B. Model 2 (2 way/230XX Airfoils/30AF)}

This was the initial model of the test program and as such problems were encountered in the initial running. The initial data obtained with this turbine showed unexpected trends with velocity ratio and variations in free-stream velocity. The problem was traced to a very small leak in the static pressure sensing system which manifested itself as an inaccurate velocity measurement. It was found that velocity measurement errors as small as .3048 ( $1 \mathrm{fps}$ ) caused large changes in power ratio, particularly at low tunnel velocities. This finding highlights the need for a very sensitive velocity setting system for wind turbine testing.

The start-up capabilities were briefly investigated with this wind turbine. The wind turbine was set at a blade angle of +20 degrees. The tunnel velocity was then gradualiy increased until the turbine started to rotate. It was observed that rotation occurred at a velocity ratio well below design. Thi3 demonstrated that a variahle pitch wind turbine could be started in relatively light winds.

\section{Model 1 (2 way/230Xx airfoils/15AF)}

This was the second of the wind turbines tested, the lowest activity factor and thus was very narrow and thin as shown in figure 9-2B. Because of its unique shape characteristics, these blades exhibited unusual blade dynamic responses while being tested. Preliminary aero structural analyses indicated that stall-flutter could occur if specified operational limits were exceeded. Indeed, several times during the running, there were audible indications that a flutter boundary had been reached. A noise similar to an operating jack hamer was heard in the tunnel which disappeared as the turbine rpm was lowered. Thus, this noise was utilized as a blade flutter indicator for subsequent testing. 
In view of its unusual operating characteristics, discussed later in the text in Section 10.4.1, a strobe light was used to observe the blade deflections. Under a stroboscopic light, it was noted that the blades were severely bowed at all operating conditions except low rpm and tunnel velocity. At one time, it appeared that one of the two blades was also flapping. Because of the extremely narrow chord of the blade it was not possible to determine if there was any torsional deflection.

Because of its small chord, this blade also operated at the lowest Reynold's number of the blades tested. To see what effect tripping the boundary layer had on performance, a strip of carborundum dust was applied to the upper surface of both blades near the leading edge. The results are discussed in Section 10.4.1.

\section{Model 3 (3 way/230XX airfoils/30AF)}

The initial running of this model led to performance levels higher than the ideal values for this turbine configuration at low velocities. It was found that the test "end-zero" point did not repeat. Ideally, the end zero point (the last test point taken after the rig and tunnel have been completely. shut-down) should agree in reading counts with the start zero point (the first test point taken before the rig and tunnel are started up). Agreement between these two readings assures that the metric system balances have not shifted during the run. Thus, it was necessary to institute a new procedure for obtaining end zeros which consisted mainly of accurately determining that the flow in the tunnel had completely stopped (zero velocity) before the reading was taken.

\section{E. Model 5 (2 way/230xx airfoils/60AF)}

This turbine was made of a wood laminate and was the most difficult to balance, requiring the most weight to bring into balance. It was found that not only did the two blades have slightly different twists, but the tips of the turbine were out of track by almost $0.127 \mathrm{~m}$ ( 0.5 inches). Nevertheless, by adjusting the blade pitch setting and careful balancing, it ran very smoothly before a blade separation occurred due to a manufacturing flaw. (Described in Appendix C)

\subsection{DATA REDUCTION AND ACCURACY}

\subsubsection{General}

The data acquired, automatically, by the STADAS data system were converted to engineering units and coefficient form and displayed on-line via the PDP- 6 computer for immediate data evaluation. In addition, these raw data were written onto a magnetic tape for more comprehensive off-line reductions. These off-line data were corrected for solid body and model wake blockage by the method of reference 19 . The corrections used are presented in Appendix $C$. A sample of the off-line data printout is shown in figures 10-4A, 10-4B, and 10-4C. Such a printout is available for each test point.

A detailed analysis of the end zero test points and a statistical analysis of the test runs were performed to determine the measurement accuracy of the data. Based on the results of this analysis, the system accuracies are presented in Table 10-2.

\subsubsection{Data Reduction}

The equations used to reduce the data are contained in Appendix C. Basically, two sets of equations are used in the reduction of the data. The first set 
is used to determine the tunnel flow conditions, the second to determine the model performance. The first set of equations used to establish the flow conditions are the standard equations used in all wind tunnels. The velocities calculated at this point are not corrected for wake or solid body blockage.

The second set of equations, designated "Model Load Conditions" accomplish two tasks. The first is to transfer the forces and.moments from the balances resolving center (BRC) to the model resolving center (MRC). After the forces and moments are transferred, the second task is to transform each component into coefficient form. All six components of force are shown in Appendix $C$ and were calculated in anticipation of yawed inflow testing. Theoretically, at zero inflow the NR, SR, MR and YR coefficients should be zero. In reality, some very small values were noted for each of these terms as can be seen in figure 10-4C. This indicates that there may have been some small pitch or yaw on the rig in the tunnel possibly due to some asymmetry in the tunnel velocity. However, these small flow angles have only a negligible effect on the primary parameters of $P R$, TR and VR presented in this report.

All of the turbine performance parameters used herein are affected by velocity (i.e. VR, TR, YR, etc.). The power ratio (PR) is effected as the cube of velocity and is therefore most sensitive to velocity inaccuracies. This is clearly shown in Table 10-3. Velocity itself is subject to residual error in primarily the differential static pressure measurement and thus the percentage velocity error increases at low speeds. The percentage error in power ratio caused by an inaccuracy in velocity is thus greatest at low velocities. To elaborate on this point, velocity is the result of stagnation pressure and temperature and a differential pressure measurement. A small differential pressure residual error results in a large velocity error at low mean velocities. An error of only $.345 \mathrm{~N} / \mathrm{m}^{2}$ (.0005 PSID) in the differential pressure measurement, results in a $.366 \mathrm{~m} / \mathrm{sec}$ (1.2 fps) error at a nominal $7.62 \mathrm{~m} / \mathrm{sec}$ (25 fps); while this pressure inaccuracy results in only a $.122 \mathrm{~m} / \mathrm{sec}$ (.4 fps) error at $21.366 \mathrm{~m} / \mathrm{sec}$ (70 fps). The nominal $7.62 \mathrm{~m} / \mathrm{sec}$ case with a $.366 \mathrm{~m} / \mathrm{sec}$ reading error would result in a power ratio error of $15 \%$; while the $.122 \mathrm{~m} / \mathrm{sec}$ error at 21.336 $\mathrm{m} / \mathrm{sec}$ would result in a power ratio error of only $1.8 \%$. This effect for a range of velocities is shown in Table 10-3.

In addition to the errors generated at low velocities due to velocity measurement residual inaccuracies, other factors deteriorate with decreasing velocity (i.e. the absolute values of torque and thrust decrease as the square of velocity). Fixed residual inaccuracies in thrust and torque for this test can result in errors at peak power ratio as indicated in Tables 10-4 and 10-5. These inaccuracies result in much larger errors in power and thrust ratios when the absolute lack of thrust and torque are lower than those corresponding to peak power ratio. The reduced data, for both reasons specified above, become more accurate with increasing tunnel velocity as the size of measurement inaccuracies decreases relative to absolute levels.

\subsubsection{Blockage Corrections}

As with all propeller and wind turbine testing, the measured velocity must be adjusted for the presence of the model support and the operation of the model itself. The corrections used are shown in Appendix B. These corrections were developed by Pope in his book "Low Speed Wind Tunnel Testing" (Ref. 19). 
Basically, two blockage corrections are involved in the adjustment of the velocity. These are the solid body blockages of the support and drive systems and the wake blockage of the wind turbine and support system.

By far the largest of these corrections is for the turbine wake blockage. This correction amounts to almost $5 \%$ of the thrust ratio and becomes larger as the thrust ratio increases.

\subsection{EXPERIMENTAL DATA PACKAGE}

The measured wind turbine performance data are presented as a data package in figures 10-5 through 10-7K. These figures include test data for the three wind turbine models tested, i.e. Mode1 1 (the 2 blade/15 AF/230XX airfoil), Model 2 (the 2 blade/30 AF/230XX airfoil) and Model 3 (the 3 blade/ $30 \mathrm{AF} / 230 \mathrm{XX}$ airfoil) wind turbines. In order to permit a direct comparison with the predicted performance, these experimental data are plotted in the same format as that shown in Section 9. Since no inflow testing was accomplished prior to the aforementioned destruction of the test rig, only curves of power ratio and thrust ratio as a function of velocity ratio are presented. On each figure, the test points have been plotted and a curve faired through the points. The model wind turbine is designated along with the run number, blade angle $(\theta 3 / 4 R)$ and the tunnel velocity. Each test point was carefully reviewed for consistency and accuracy of measurements. Those points which scattered outside of the measurement tolerance were indicated and generally have not been included in the analysis procedure. All of the test data except for tare runs, and where instrumentation problems occurred are presented in this section. The discussion at this point will be limited to an evaluation of the test data. The data analysis and correlation with calculations will be covered in Section 11.

\subsubsection{Model 1}

A photograph of Model 1 (2 blade/15 AF) as mounted in the wind tunnel is shown in figure 10-5A. The measured performance data is shown in figures 10-5B through 10-5E. For the design blade angle of $-1.0 \circ, \theta 3 / 4 \mathrm{R}$, the variation of power ratio with velocity ratio is plotted in figure 10-5B for three tunnel velocities. For runs number 15 and 18 the data were adjusted manually to account for a s.tart-zero shift in dynamic pressure, H-P, corresponding to $-.345 \mathrm{~N} / \mathrm{m}^{2}(-.0005 \mathrm{PSIG})$ and $-.138 \mathrm{~N} / \mathrm{m}^{2}(-.0002 \mathrm{PSIG})$ respectively. These shifts in the H-P start-zero are believed to be caused by residual airflow in the test section while start-zeroes were being acquired which would cause velocity readings to be lower than actual test conditions for all data points during these runs. As can be seen from an inspection of figure 10-5B, there is little scatter of the test points within a run. The same general observations can be made for the corresponding thrust ratio data shown in figure 10-5C. For no established reason, the thrust data of run $\$ 15$ appears to be out of line with respect to the other data. As discussed previously in Section 9 , the tests were run at several different velocities to achieve maximum R.N. at peak P.R. and to cover the complete V.R. range at one velocity. Since $R_{\circ} N$. is a function of velocity and increases with velocity increase, it can be seen that the measured data show a variation with R.N.' In the higher V.R. region, the P.R. increased with decreasing R.N. (i.e. velocity). This trend is inconsistent with the corresponding calculated data presented in Section 9 
and with the well established effects of Reynolds number in fluid dynamics. Probably the trend is due to the previously discussed measurement inaccuracies which nearly always result in the actual velocity being greater than the recorded values. Moreover, the difference between recorded and actual velocity increases as the recorded velocity is decreased. Thus, it is obvious that the associated power ratio and thrust ratio levels will show the observed trend. Furthermore, as indicated in Section 9, the predicted $R . N$. effect on performance for the small spread in tunnel velocity is small and generally would be observed within the data accuracy band. In the lower V.R. region the measured data show a trend with R.N. as predicted in Section 9, In this case the observed trend with velocity is probably due to a stronger R.N. effect than predicted. Since the stall angle of attack of an airfoil section decreases as R.N. decreases, there is an abrupt fall off in performance as the R.N. is decreased. To better understand this situation, a cursory at tempt was made to apply roughness to the leading edge radius in an attempt to simulate full scale $R_{0} N$. Accordingly a number 360 grit was applied to a band approximately .032 meter (.125 inch) wide at $5 \%$ chord on the suction side of the leading edge. It can be seen from run $\mid ⿰ 21$ that although the leading edge roughness significantly reduced performance at the higher V.R. range, it did alleviate the abrupt fall-off in power ratio ( $R \circ N$. effect) at the low V.R. range. Thus while the grit apparently did increase the blade stall angle-ofattack it is implied that the grit was applied too thickly and introduced grit drag which reduced performance. Nevertheless, the experiment does indicate that there is a potential of simulating full scale R.N. in small scale tests with proper definition of grit size and location on the leading edge.

Data for the blade angle range tested to define variable pitch characteristics are shown on figures $10-5 D$ and 10-5E. Run \#18 from figures 10-5B and 10-5C was selected as the design blade angle. Similar trends with R.N. (i.e. velocity) are seen for the $.5^{\circ}$ blade angle as for the design angle. The drop-off in P.R. level with increases in blade angle is shown to be as predicted in Section 9.

It was observed when running the 2 blade/15 AF model that the blades bowed significantly due to the thrust loading. Therefore, a strobe light was used to better observe the phenomena. It was seen that flatwise bending and flapping were encountered at some combinations of velocity and RPM on one blade with steady bending of the other blade. This bending decreased with increasing blade angle and in general as loading decreased. In view of the observed blade deflection, the reliability of these data is questionable, particularly at the design angle where slight variation in blade twist distribution could cause a large change in performance, particularly near the free running velocity ratio.

\subsubsection{Model 2}

Model 2 (the 2 blade/30 AF) is shown as mounted in the wind tunnel in figure $10-6 \mathrm{~A}$ and the measured data is presented in figure 10-6B through 10-6E. For the R.N. variation investigation, the $1^{\circ}$ blade angle was selected over the $-.5^{\circ}$ predicted design blade angle because it shows better performance over a wider V.R. range (figure 10-6D) albeit with a slight penalty at peak P.R. The test data for a range of velocities is shown in figures $10-6 B$ and $10-6 C$. As was seen with model 1 , scatter within a run 
is slight and the same R.N. trends as discussed for model 1 were also seen for model 2. Again, as previously discussed, the validity of P.R. and T.R. plots are suspect due to the sensitivity to small errors in velocity measurement.

The complete blade angle range is shown as figure $10-6 \mathrm{D}$ and $10-6 \mathrm{E}$. The $11 \mathrm{~m} / \mathrm{S}$ ( $37 \mathrm{FPS}$ ) $1^{\circ}$ blade angle curve was repeated on these figures. It is of interest to note that for the $5^{\circ}$ and $10^{\circ}$ blade angles there are two runs each at high velocities which show a slight increase in peak $P . R$. with R.N. (i.e. increase in velocity) as predicted. As previously discussed, the accuracy of measuring improves as velocity increases. Moreover, the effect of R.N. at the lower V.R. range is considerably less than that observed at the $1^{\circ}$ blade angle runs corresponding to lower velocities and consequently lower $R_{0} N_{0}$, a further indication that the spread in P.R. at the lower V.R. range is due to R.N.

\subsubsection{Model 3}

More data were obtained for Model 3 (the 3 blade/30 AF) than the other two models. The configuration as mounted in the wind tunnel is shown on figure 10-7A. For $1^{\circ}$ blade angle, several runs were made at three velocities. The P.R. and T.R. data are presented in figures $10-7 \mathrm{~B}$ and 10-7C respectively for $8 \mathrm{~m} / \mathrm{s}$ (26 FPS) for four runs. Again, as pointed out for the other two models, the scatter within a run is small except for run \#29. Moreover, an examination of the test data indicates that a transducer problem was encountered in run $\$$ 非 29 which resulted in erroneous performance data. Thus this run should be ignored. Run \#28 appears to be incorrect. In this run there was an indication that the tunnel fan RPM was higher than for any other $8 \mathrm{~m} / \mathrm{s}$ (26 FPS) run and thus lends credence to suspecting its accuracy. For a velocity of $14 \mathrm{~m} / \mathrm{s}$ (46 FPS) the data for three runs are shown in figure 10-7D for P.R. and 10-7E for T.R. With similar data being plotted in figures 10-7F and 10-7G for $22 \mathrm{~m} / \mathrm{s}$ (73 FPS). It should be noted that for each velocity, the spread in the P.R. and T.R. plots for the run becomes smaller as velocity is increased and is well within the accuracy bands as previously defined.

The average P.R. and T.R. of the runs at each velocity for the $1^{0}$ blade angle are shown in figures 10-7H and 10-7I. It can be seen from these figures, that the averaged data tends to offset the measurement inaccuracies previously noted.

The variable pitch data are presented in figures $10-7 \mathrm{~J}$ and $10-7 \mathrm{~K}$ for P.R. and T.R. respectively. The $1^{\circ}$ blade angle is represented on these figures by the $14 \mathrm{~m} / \mathrm{s}$ ( $46 \mathrm{FPS}$ ) average from figures $10-7 \mathrm{H}$ and 10-7I. As for model 2 , at $5^{\circ}$ and $10^{\circ}$ blade angles, peak P.R. increased with increased R.N. and the drop-off at the lower V.R. is less severe than at the lower blade angles run at lower velocities.

In summary, the measured data of model 3 has a high degree of reliability because the many reruns permitted fairing the data which tends to average out the measurement inaccuracies previously noted. Although there were no reruns for model 2, most of the data were taken at velocities where the inaccuracies are small and except at the lowest velocity, these data are considered to be quite reliable. Model 1 data are the least reliable because of the blade instabilities noted during the testing and discussed above. 
In view of the limited number of model wind turbine configurations tested in this program due to the previously reported accident, the inclusion of supplemental test data on model wind turbines from other programs becomes important in achieving the objectives of this program. Thus, the literature search discussed in Section 5 becomes important in providing this supplemental data. As pointed out in Section 5, very little experimental data on model wind turbines comparable to the models included in this test program are available. However, the test data presented in references 20 and 21 have been selected to supplement the test data from this program.

Reference 20 contains performance data on a series of .9 and 1.2 meter ( 3 and $4 \mathrm{ft.}$ ) wind turbine models tested in the New York University. wind tunnel. Reference 21 contains unpublished data in several 1.8 meter ( $6 \mathrm{ft.}$ ) diameter wind turbine rotors. Both of these test programs are described in more detail below.

\subsubsection{New York University Data}

In reference 20, 2-bladed rotors of .9 meter $(3 \mathrm{ft.})$ and 1.2 meter ( $4 \mathrm{ft.}$ ) were tested in the $2 \times 3$ meter $(7 \times 10 \mathrm{ft}$.$) tunnel at N.Y.U.$ on a special test rig. The rig was mounted in the center of the tunnel and consisted of a spinner, hub and motor-generator mounted in a streamlined fairing. The motor-generator was a shunt-wound D.C. motor of $2.2 \mathrm{~kW}$ ( 3 H.P.) (nominal) and was rigged to permit use either as a motor to drive the model wind turbines or as a generator to absorb the power produced by the wind turbines.

Four sets of wooden blades were available for testing on this rig. The first set of blades was designated Model A. This rectangular 97 AF blade was a scale model of the Smith-Putnam wind turbine and represented a. 9 meter ( $3 \mathrm{ft}$. ) model of that wind turbine. The second set of blades was a $50 \mathrm{AF}$ rectangular set designated Model B. The third and fourth models incorporated tapered blades of $34 \mathrm{AF}$, and were identical in all respects except for twist. These models were designated $\mathrm{C}-1$ and $\mathrm{C}-2$ respectively with the $\mathrm{C}-2$ blade design having the more optimum twist distribution. Models $B, C-1$ and $C-2$ resulted in wind turbines of 1.2 meter ( $4 \mathrm{ft.}$ ) dlameter.

of these four model wind turbines, only two received extensive testing due to manufacturing and structural problens encountered with the small wooden blades. The testing of model $B$ was limited diye to a ocvere flutter problem encountered under operating conditions. Model C-1 blade had less than optimum twist in the critical inboard regions and therefore received only minimal testing. Models A and C-2 received more extenstve testing, covering a range of tip speeds, forward velocities and blade angles. However, prior to the test, it was discovered that the two $\mathrm{C}-2$ blades had different twist distributions. These blades were acceptable from the hub to the .457 meter (18 inch) radius but the remaining .15 meter ( 6 inch) of the blades were quite badly warped, the tip of one blade having $3^{\circ}$ over twist and the other one having $6^{\circ}$ under twist due to warpage after manufacturing. In spite of this twist mis-match, the $\mathrm{C}-2$ wind turbine was tested over a range of blade angles, forward velocities and tip speeds. 
A representative sample of the data from this test is shown in figures 10-8, 10-9, and 10-10. Figure 10-8 shows the variation of power ratio with changes in freestream velocity for Model $A$. The trends shown here are similar to those observed in the present test; i.e. the more abrupt fall-off in power ratio at lower velocity ratios with decreasing velocity. The data shown in this figure are for the same blade angle. However, similar data at other blade angles show the same trend.

Figure 10-9 shows the variation of power ratio with blade angle for Model $\mathrm{C}-2$. Again these trends are similar to those observed during the present test.

A summary of the performance of all four model wind turbines tested at N.Y.U. is shown in figure 10-10. This figure presents the peak performance of all four models. The rather poor performance of Models $\mathrm{B}, \mathrm{C}-1$ and $\mathrm{C}-2$ compared to Model $\mathrm{A}$, is immediately apparent. As will be recalled, Model $B$ had severe flutter problems, Model $C-1$ had less than optimum twist while Model $\mathrm{C}-2$ had blades with mis-matched twists. Moreover, all of these models were tested at Reynolds of approximately $.300 \times 10^{6}$ which may have contributed to the low performance levels. The data are included with the test data acquired in this test program and described in Section 11.

\subsubsection{Sulzer Unpublished Data}

Recently, P. Sulzer (ref. 21) tested several 1.83 meter (6 ft.) diameter wind turbine models on his test rig. This rig is unique in that a motor boat is used to propel the wind turbine models over the water at a constant $11 \mathrm{~m} / \mathrm{s}$ ( $37 \mathrm{fps}$ ). The model is mounted on a motor generator .9 meter ( 3 $\mathrm{ft.}$ ) in front and 1.52 meter $(5 \mathrm{ft}$ ) above the bow of the boat. In making a test run, the boat is operated at a constant wind speed of $11 \mathrm{~m} / \mathrm{s}$ ( $37 \mathrm{fps}$ ) as indicated by an anemometer located 1.52 meters ( $5 \mathrm{ft}$ ) in front of the wind turbine model. The model is started with a 12 volt starter motor that can then be mechanically disengaged from the drive shaft. The model is then adjusted to the desired RPM with a Varicon-controlled load. At that point, the load power and RPM are recorded. This procedure is repeated until all blade angles have been investigated.

The wind turbine models tested on this rig are 1.83 meters (6 ft.) in diameter. Turbines with rectangular and tapered planforms have been tested with most of the turbines utilizing the GA(W)-1 airfoil. A constant blade thickness ratio of 17 percent is used regardless of the blade planform. A total of nine wind turbines have been tested on this rig. The results of the six most appropriate turbine tests are presented herein.

The data from this rig are shown in figures 10-11 and 10-12. In figure 10-11, the performance of 47 and $77 \mathrm{AF}$ tapered blade models are compared. Also included is one test point for a wind turbine with $74 \mathrm{AF}$ rectangular blade. In figure 10-12, the performance of one and two bladed, $47 \mathrm{AF}$ wind turbines is shown. Superimposed is the performance of a $46 \mathrm{AF}$ rectangular one bladed wind turbine. Recalling the previous figure, the trapezoid planform blades show higher performance.

All of these data will be discussed and compared with the results of the present program in Section 11. 
RUN SCHFDULE

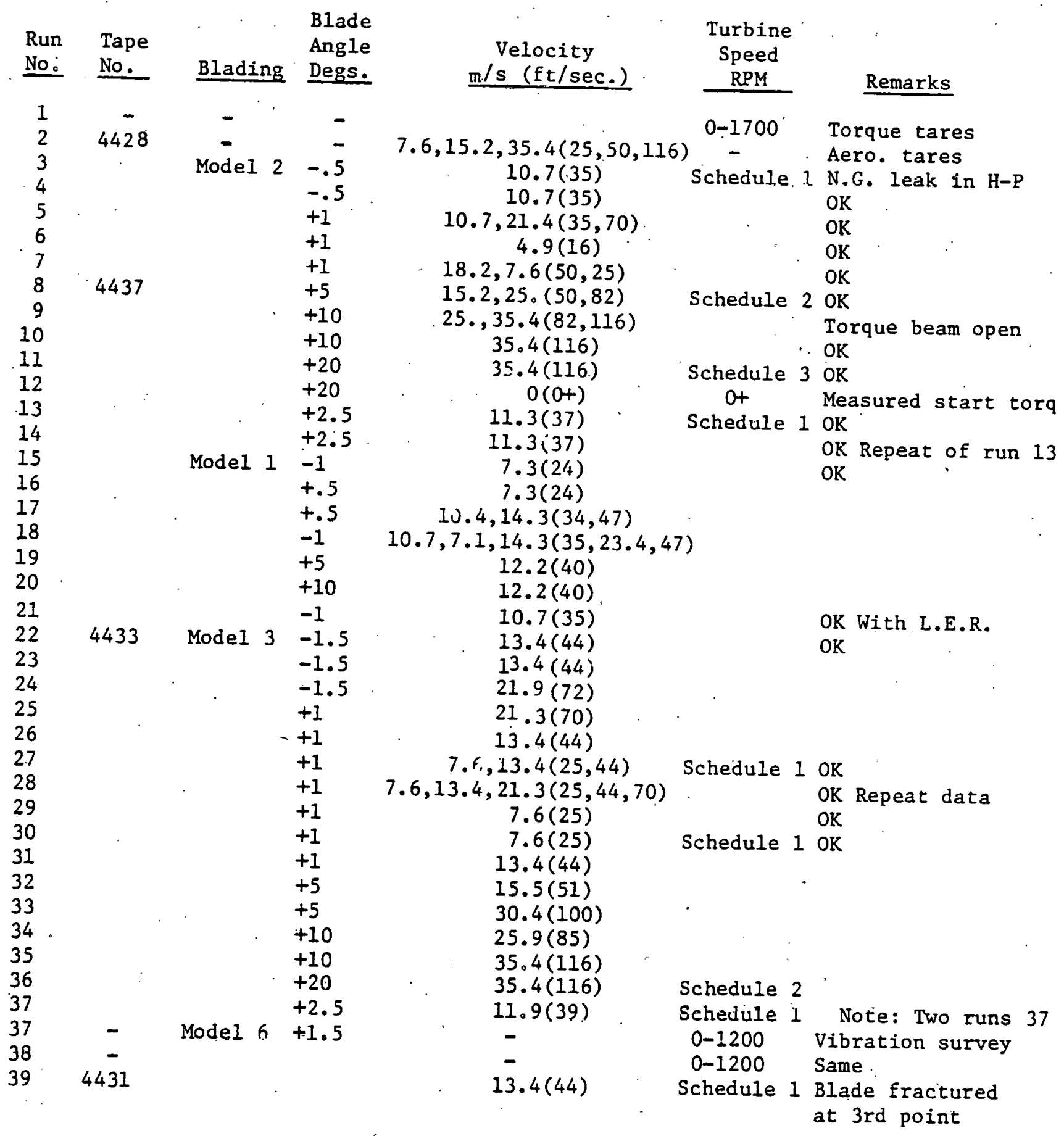

Turbine Speed Variation Schedules

\begin{tabular}{rlll}
\hline Schedule & 1 & 1671, & $1433,1194,955,716,475$ \\
2 & $1671,1433,1194,955,716,400$ \\
3 & $1194,954,716,475,400$
\end{tabular}




\section{TABLE $10-2$}

\section{Statement of Accuracies}

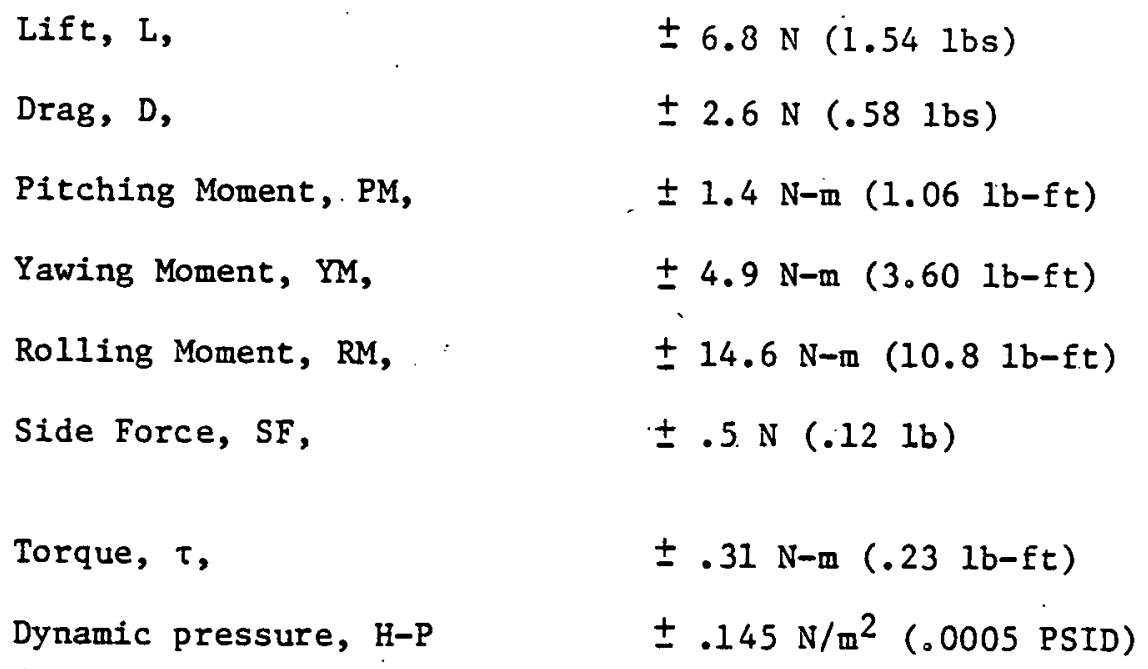


TABLE $10-3$

Effect of Velocity Measurement Accuracy on Power Ratio

$\mathrm{m} / \mathrm{s}$ (FPS)
$35(116)$
$21(70)$
$14(45)$
$8(25)$
$5(17)$

35 (116)

21 (70)

5 (17)

\section{Nominal Velocity}

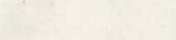

\section{Accuracy}

$\mathrm{m} / \mathrm{s}$ (FPS)

$\pm 0.1(0.3)$

$\pm 0.1(0.4)$

$\pm 0.2(0.6)$

$\pm 0.4(1.2)$

$\pm 0.6(2.0)$

\section{Effect on PR}

$\%$

$\pm 1$

$\pm 2$

$\pm 4$

$\pm 15$

$\pm 40$

\section{TABLE $10-4$}

Effect of Power Measurement Accuracy on Power Ratio $\pm[.31 \mathrm{~N}-\mathrm{m}(.231 \mathrm{~b}-\mathrm{i} t)]$

Nominal Velocity

$$
\begin{array}{cl}
\mathrm{m} / \mathrm{s} & \text { (FPS) } \\
14 & (45) \\
8 & (25) \\
5 & (17)
\end{array}
$$

\section{Effect on Peak PR}

$$
\begin{aligned}
& \% \\
& \pm 1 \\
& \pm 5 \\
& \pm 8
\end{aligned}
$$

TABLE $10-5$

Effect of Thrust Measurement Accuracy on Thrust Ratio $\pm[2.6 \mathrm{~N}(.58 \mathrm{lbs})]$

Nominal Velocity

$\mathrm{m} / \mathrm{s}$ (FPS)

14 (45)

8 (25)

5 (17)

\section{Effect on I므}

\section{$\%$}

$\pm .5$

$\pm 2$ 


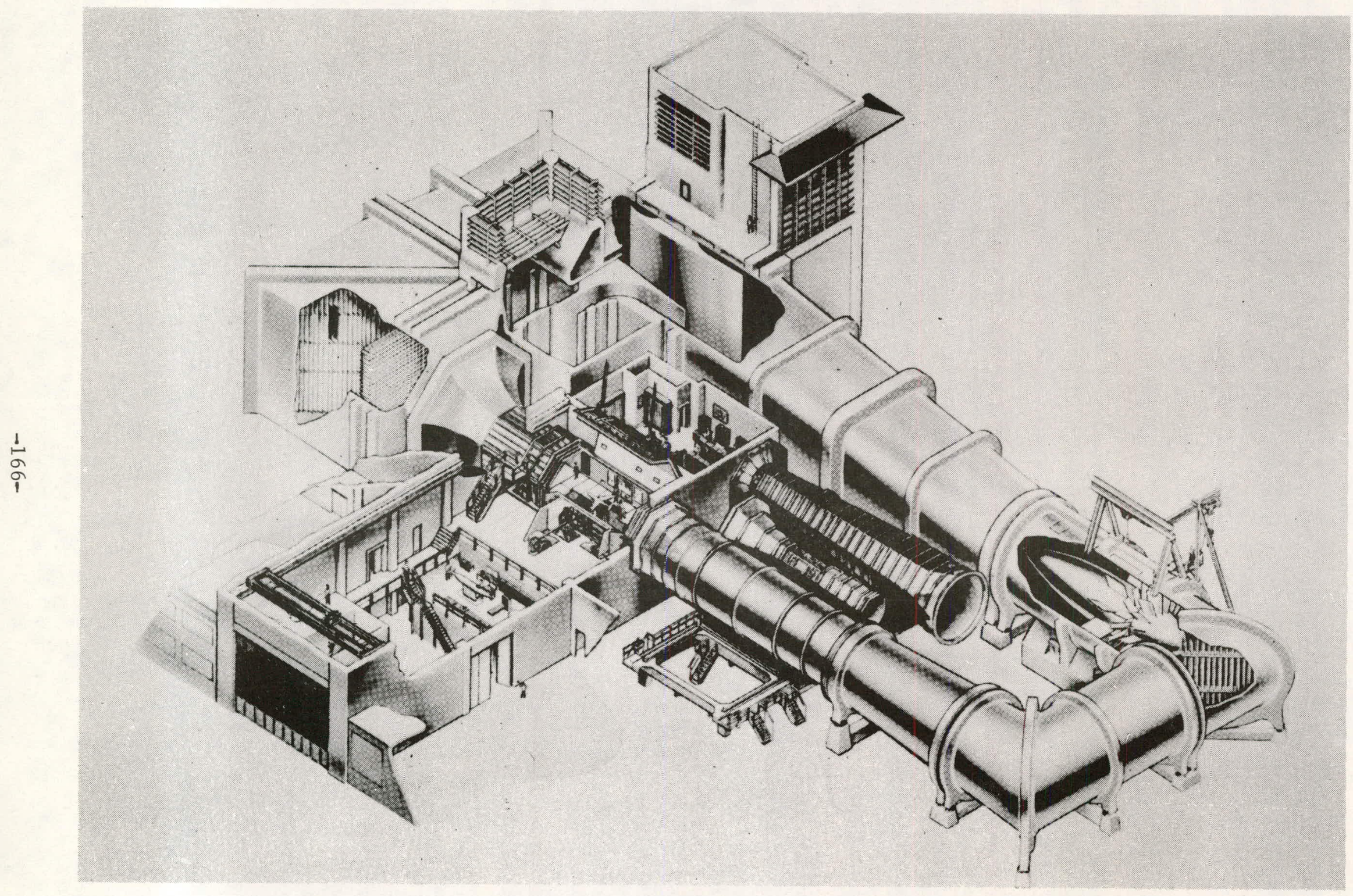

FIGURE 10-1. UTRC WIND TUNNEL TEST FACILITY 

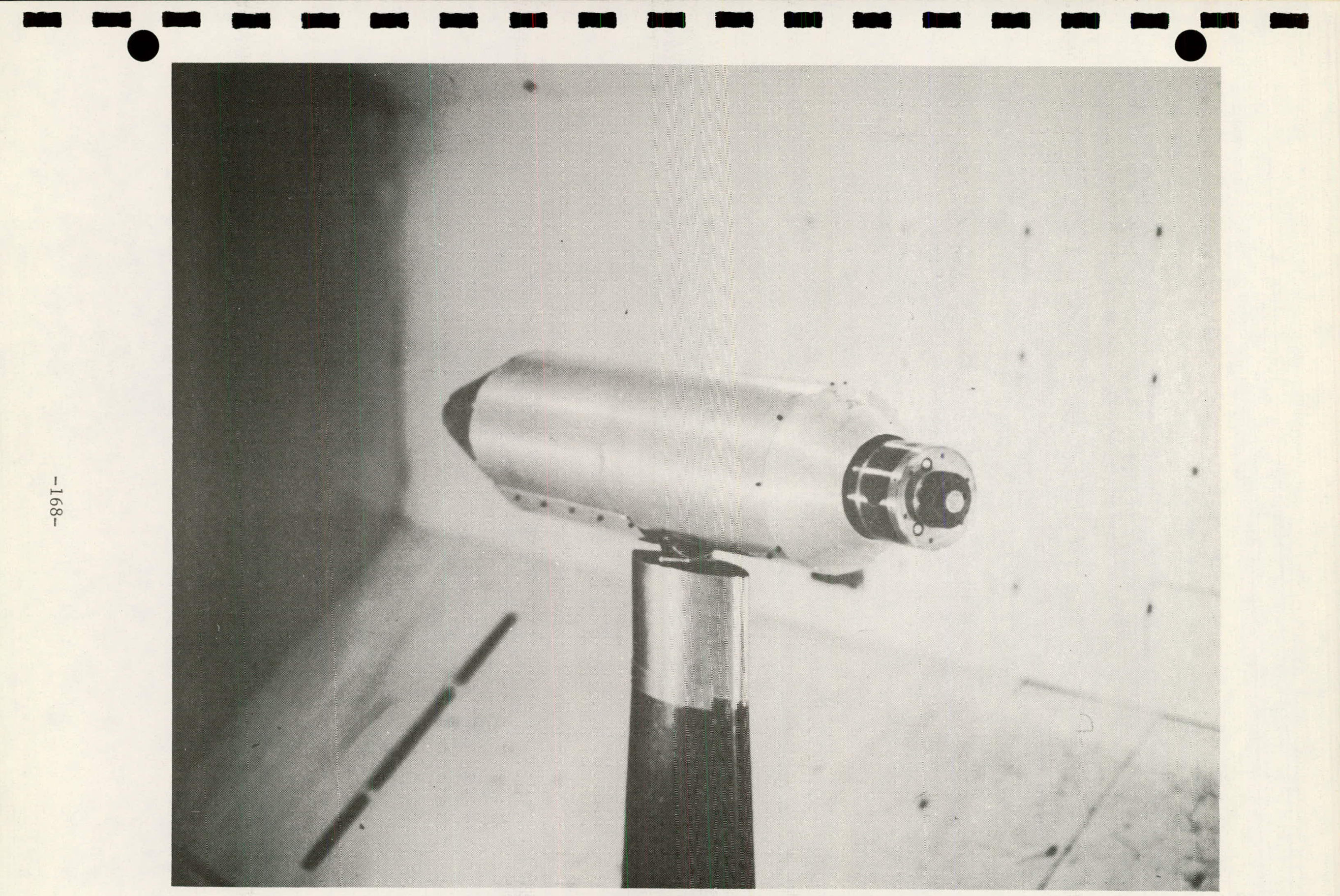

FIGURE 10-3. TEST RIG IN 5.48 METER (18 FOOT) TUNNEL 
STADAS TEMPERATURE ANO PRESSURE BASE PROGRAM

SAd OARA (Ten torque readings in system units; ft-lbs/非 units)

ruA 5 POINT 5

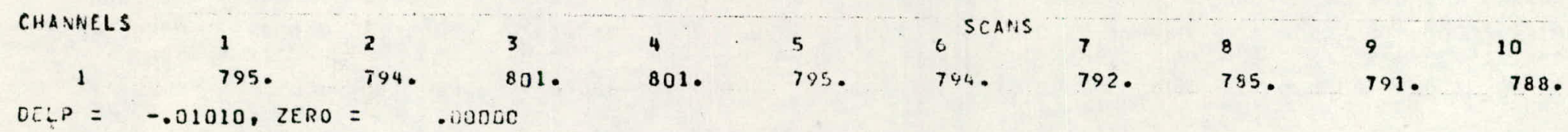

FIGURE 10-4A RAW TORQQUE INPUTS 
SALE ENGINEERING UNITS

RUv 5 POINT 5 (Ten torque readings corrected to $\mathrm{ft}-1 \mathrm{bs}$ )

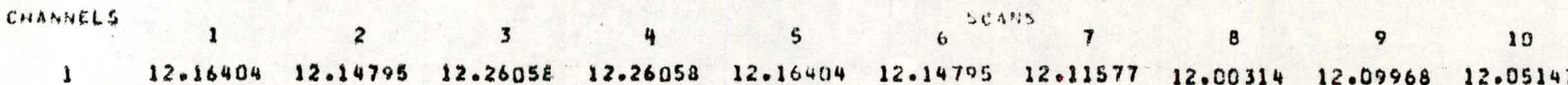

PARAMETER BOARO (Run constants input)

100000001000100200300013200000

$\begin{array}{lllllllllllllllllllllllll}n & 1 & 0 & 0 & 1 & 0 & 9 & 0 & 3 & 1 & 0 & 7 & 0 & 9 & 0 & 0 & 0 & 1 & 0 & 0 & 8 & 1 & 6 & 8 & 9 \\ 9 & 0 & 2 & 2 & 2 & 6 & 0 & 0 & 0 & 0 & 0 & 6 & 0 & 0 & 8 & 0 & 3 & 2 & 9 & 4 & 3 & 0 & 4 & 0 & 0\end{array}$

COMPUIEO DATA (Uncorrected torque, average of 10 readings, ft-1bs)

TOROU

12.14151

RUN CONSTANTS (Balance and transducer calibrations, slopes and distances)

\begin{tabular}{|c|c|c|c|c|c|c|c|c|c|c|c|}
\hline $\begin{array}{l}0 A T S L \\
1.00000\end{array}$ & DSF & LSF 1.30003 & $S F S_{1.00000}$ & $\begin{array}{l}\text { PRNTL } \\
\text { 1.0כก00 }\end{array}$ & $\begin{array}{l}D E \\
-.01010\end{array}$ & 42.82000 & $\begin{array}{l}\text { Trun } \\
76.50000\end{array}$ & $\begin{array}{l}\text { PCALL } \\
\text { COOOOO }\end{array}$ & $\begin{array}{l}\text { PCLI } \\
.00000\end{array}$ & $\begin{array}{l}\text { PCL2 } \\
.00000 !\end{array}$ & PCL 30000 \\
\hline $\begin{array}{l}\text { PPY } \\
953.00000\end{array}$ & ${ }^{R} 4.00000$ & ${ }_{3}^{R} .000013$ & $\begin{array}{l}x 0 \\
-2.63540\end{array}$ & ${ }^{\text {Yo }} .00000$ & 20.00000 & $\times 1.00000$ & 28.00000 & $\stackrel{\text { PHI }}{.0}$ & $\begin{array}{l}\text { PSIC } \\
30.000\end{array}$ & $\begin{array}{l}\text { SPMM } \\
8.00000\end{array}$ & SP \\
\hline $\begin{array}{l}\text { SPMP } \\
9.00000\end{array}$ & $\begin{array}{l}\text { SRMM } \\
3.00000\end{array}$ & $\begin{array}{l}\text { SRMC } \\
.00000\end{array}$ & $\begin{array}{l}\text { S.ivip } \\
9.05000\end{array}$ & $\begin{array}{l}\text { PMH } \\
168.90000\end{array}$ & $\begin{array}{l}\text { PUC } \\
1.00000\end{array}$ & $\begin{array}{l}\text { PHP } \\
320.70000\end{array}$ & $\begin{array}{l}\text { RMM } \\
329.40000\end{array}$ & $\begin{array}{l}R N O \\
6.30000\end{array}$ & $\begin{array}{l}\text { RMP } \\
222.60000\end{array}$ & $\begin{array}{l}\times K O \\
1.000\end{array}$ & $P C$. \\
\hline TS .00000 & 0000 & $\begin{array}{l}A F \\
30.00000\end{array}$ & .16700 & $\begin{array}{l}\text { TIARE } \\
\text {-.80000 }\end{array}$ & 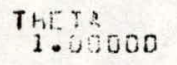 & $\begin{array}{l}\text { Thef } \\
\text { 1. }\end{array}$ & PSI & ALPH & & & \\
\hline
\end{tabular}

ENCODER DATA (Six component balance readings, 1 ift and drag in $1 \mathrm{bs}$, moments in ft.

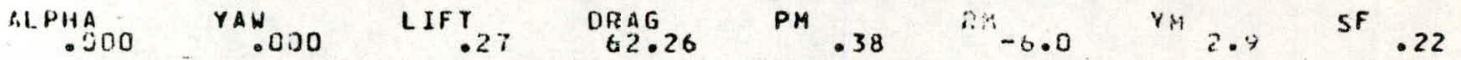


HSO WIND TURBINE TEST

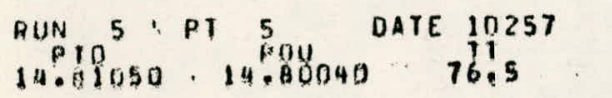

RPH Tद्व

"50BALANCE RESOLVING

$$
\begin{aligned}
& \text { BALANCE RESOLVING CENTER } \\
& \begin{array}{lll}
\text { L.AN } & \text { D } \\
.27 & 61.67 & .38
\end{array}
\end{aligned}
$$

PHSMY

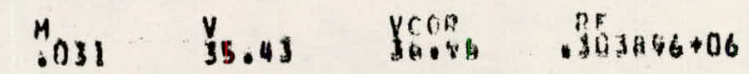

RMSMY PSI

$\begin{array}{lll}Y M & R M & S F \\ 2.91 & -6.02 & .22\end{array}$

**300r axIS

$\begin{array}{lll}i .27 & 0 & P M \\ 1.67 & 1.10\end{array}$

$\begin{array}{cll}\text { TR } & \text { NR } & \text { SR } \\ -.757 & .043 & .003\end{array}$

YM

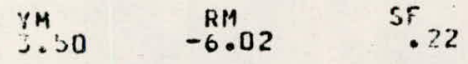

$\begin{array}{ccccc}I & A L F H A & B & A F & V T I P \\ V & 1.0 & 2 & 30 & 349.19\end{array}$

RHOU $=.002376$
FIGURE 10-4C FINAL DATA OUTPUT

(Terms defined in List of Symbols) 


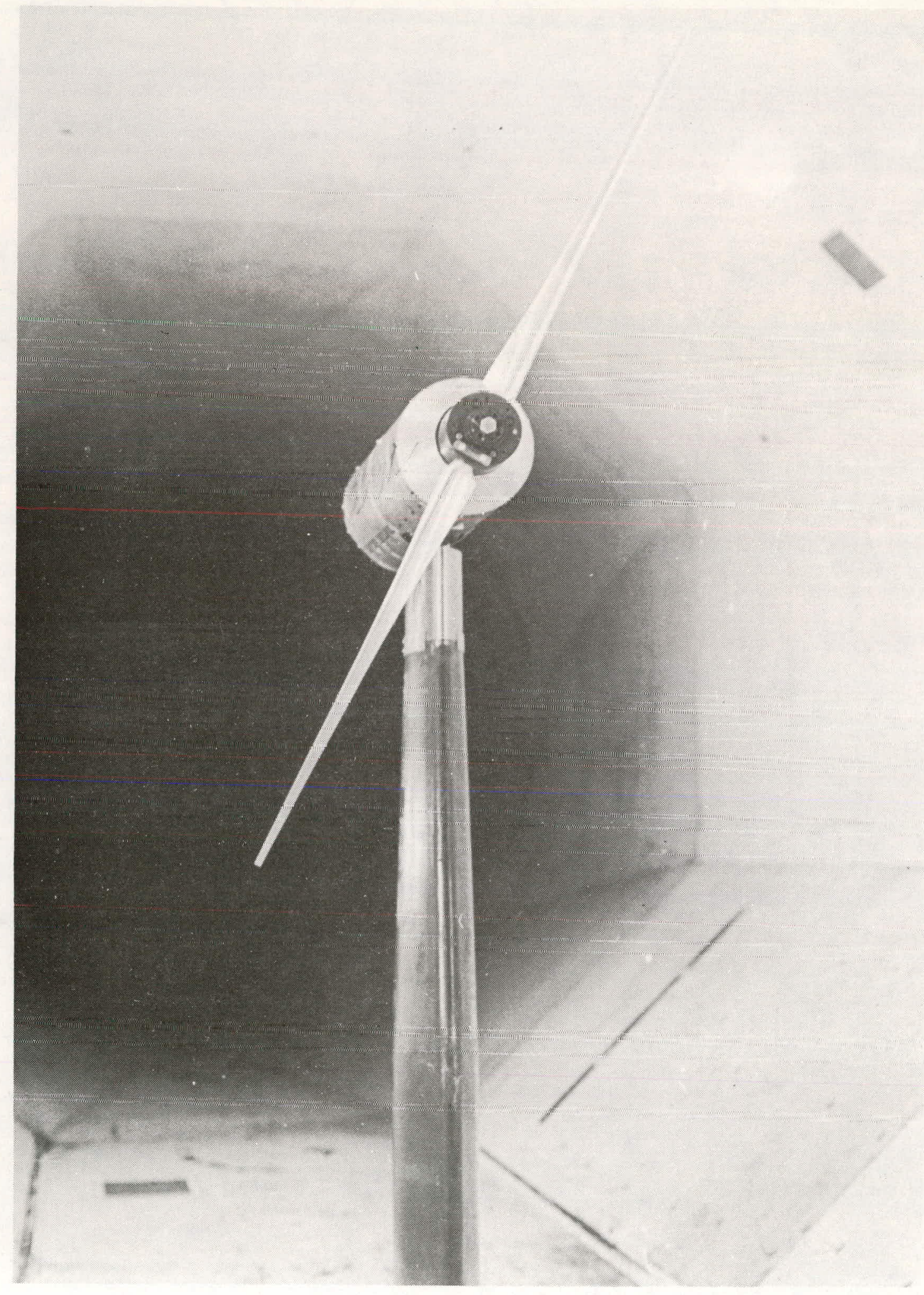

FIGURE 10-5A. 15AF MODEL NO. 1IN 5.48 METER (18 FOOT) TUNNEL 

MODEL NO. 1

15 ACTIVITY FACTOR

NACA 230XX AIRFOIL SECTIONS

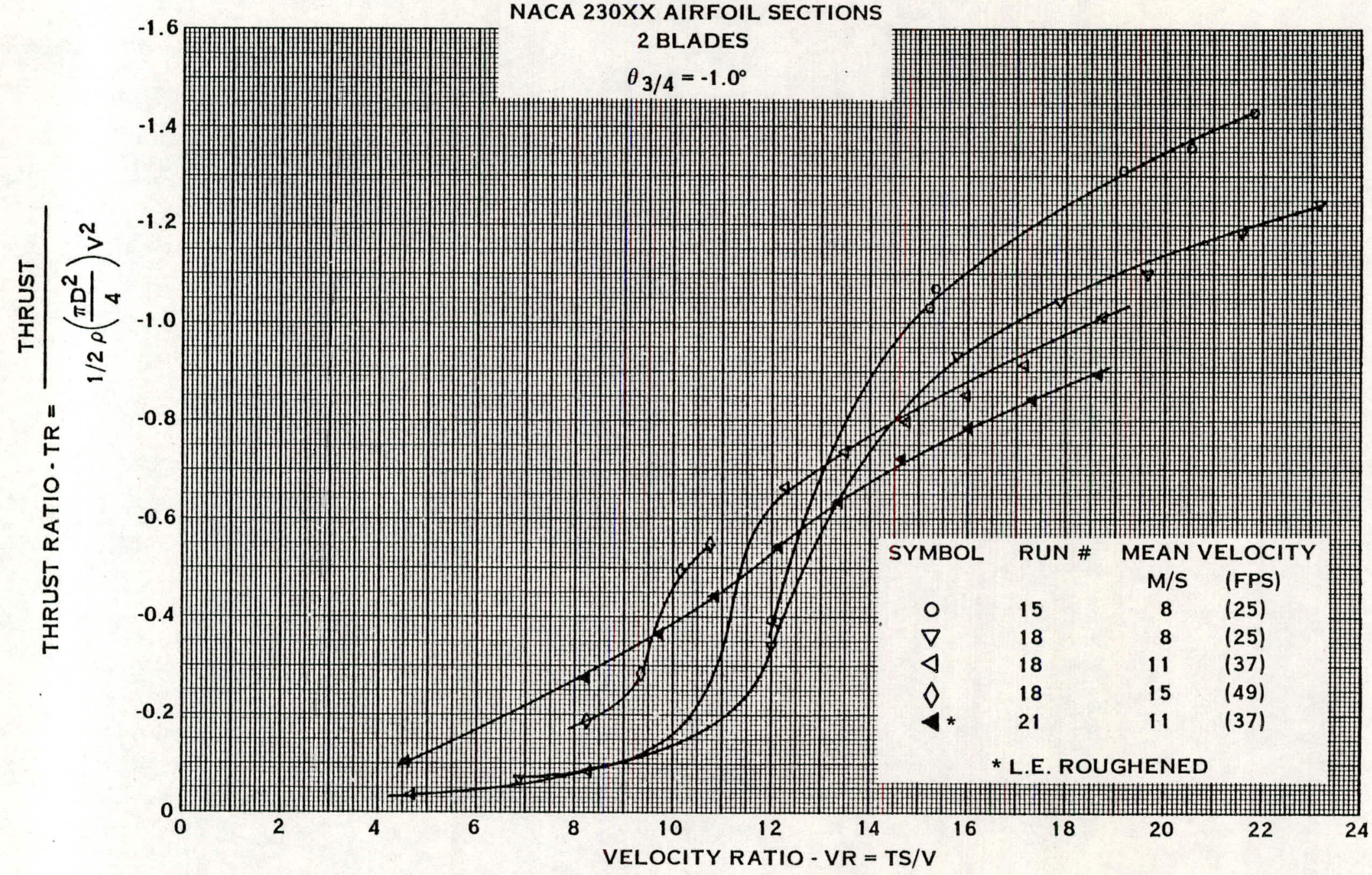

FIGURE 10-5C. EFFECT OF VELOCITY ON MEASURED THRUST RATIO 


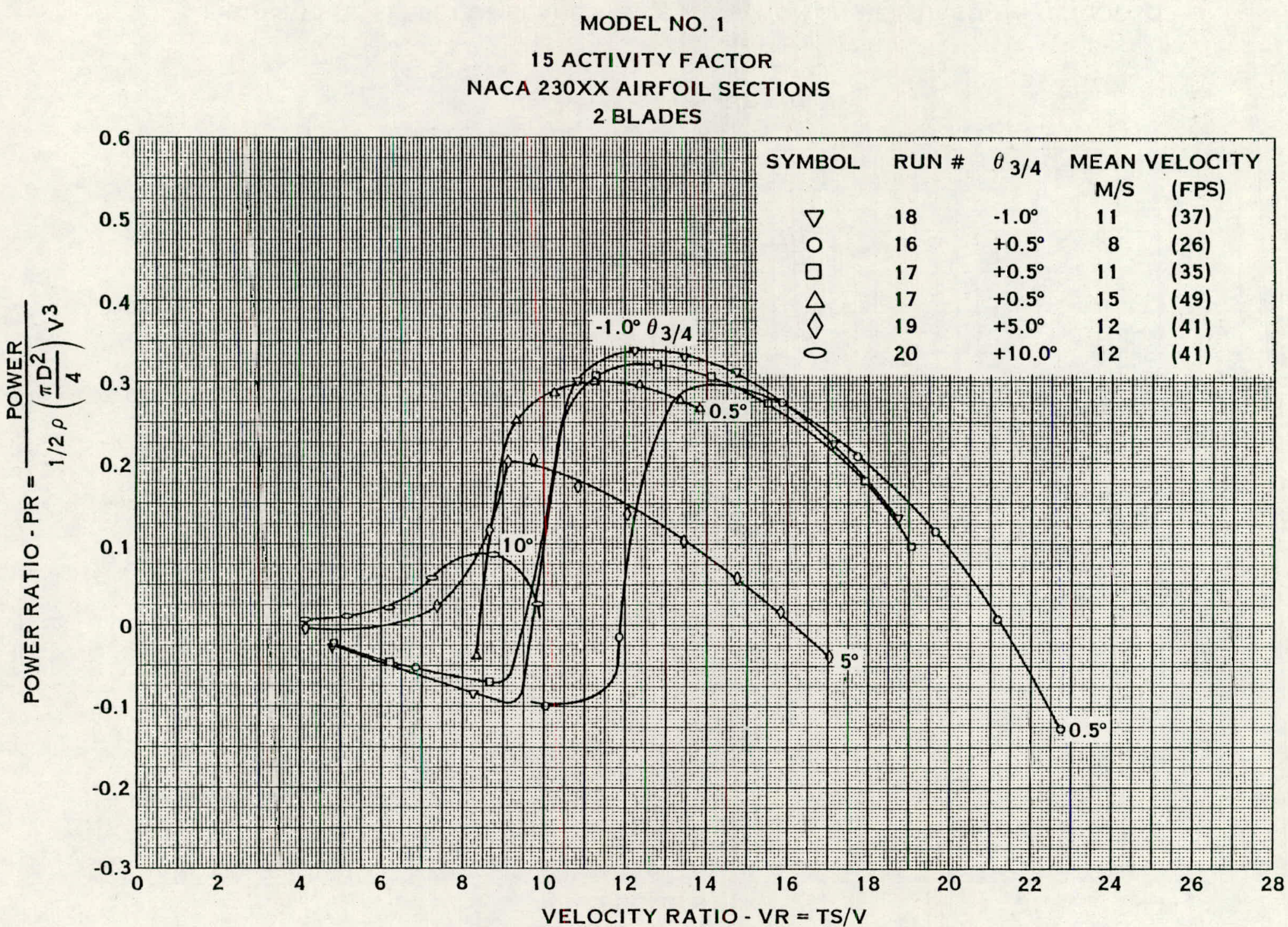

FIGURE 10-5D. EFFECT OF BLADE ANGLE VARIATION ON MEASURED POWER RATIO 
MODEL NO. 1

15 ACTIVITY FACTOR

NACA 230XX AIRFOIL SECTIONS

2 BLADES

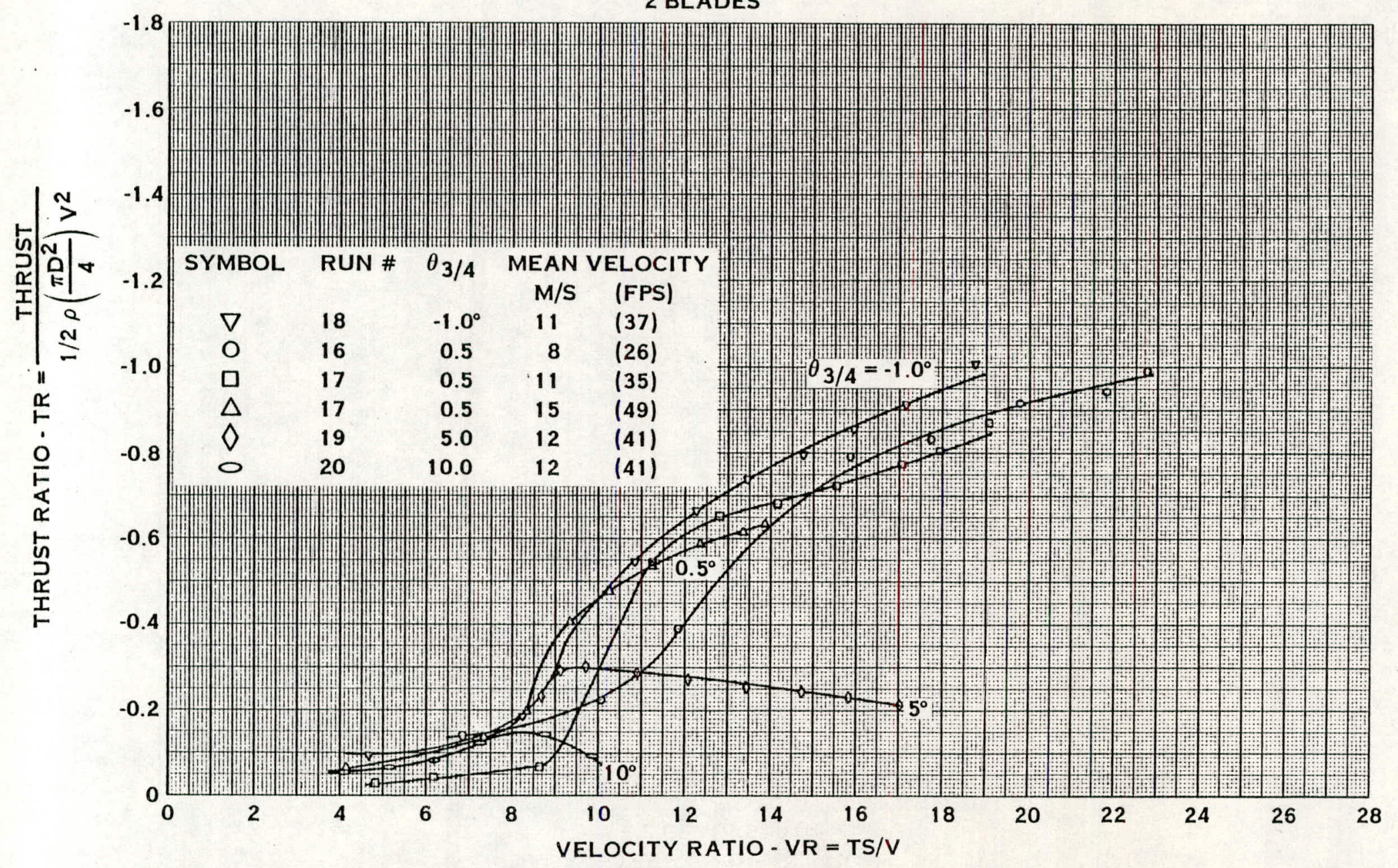

FIGURE 10-5E. EFFECT OF BLADE ANGLE VARIATION ON MEASURED THRUST RATIO 


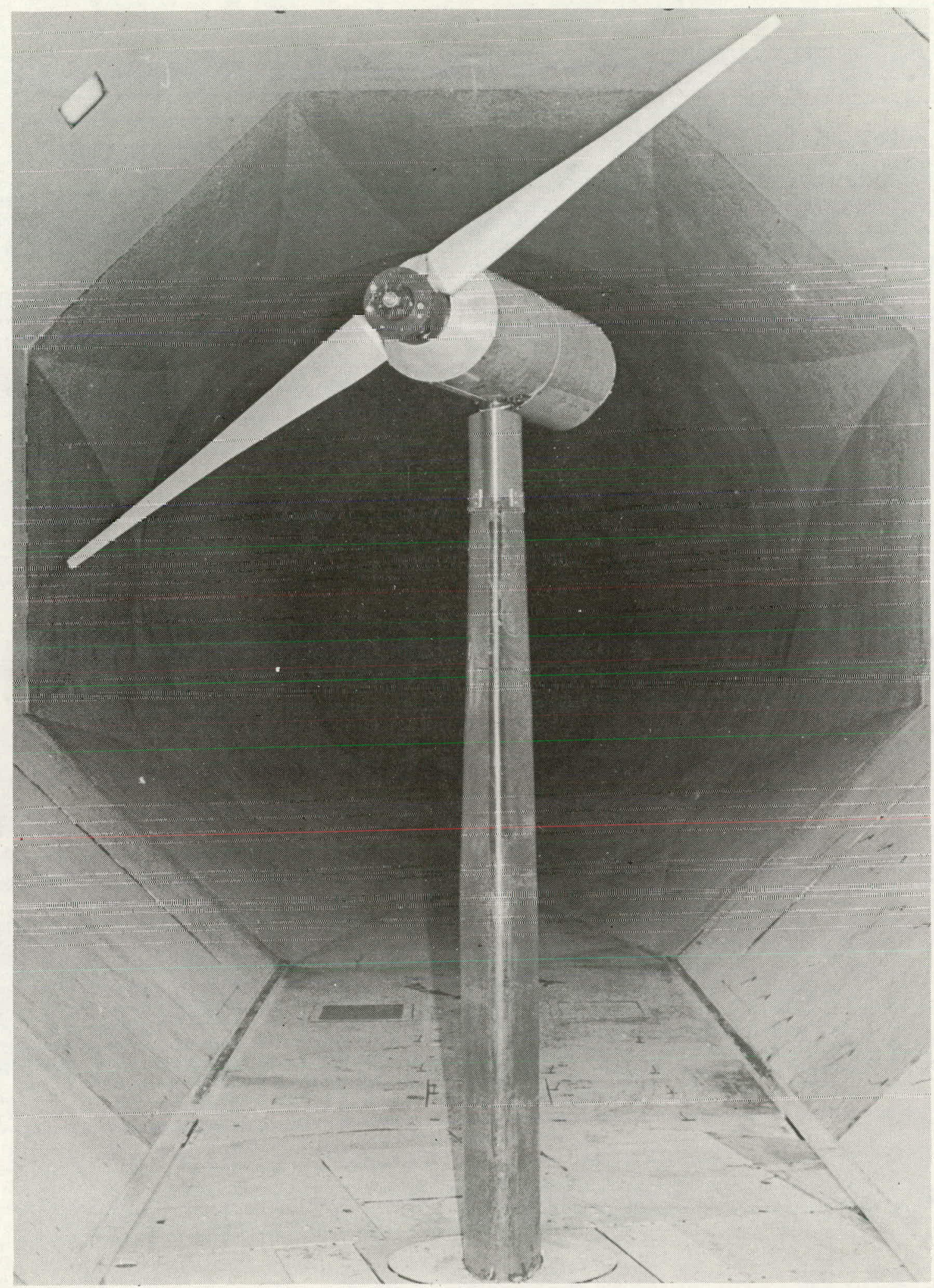

FIGURE 10-6A. 30AF MODEL NO. 2 IN 5.48 METER (18 FOOT) TUNNEL 
MODEL NO. 2

30 ACTIVITY FACTOR

NACA 230XX AIRFOIL SECTIONS

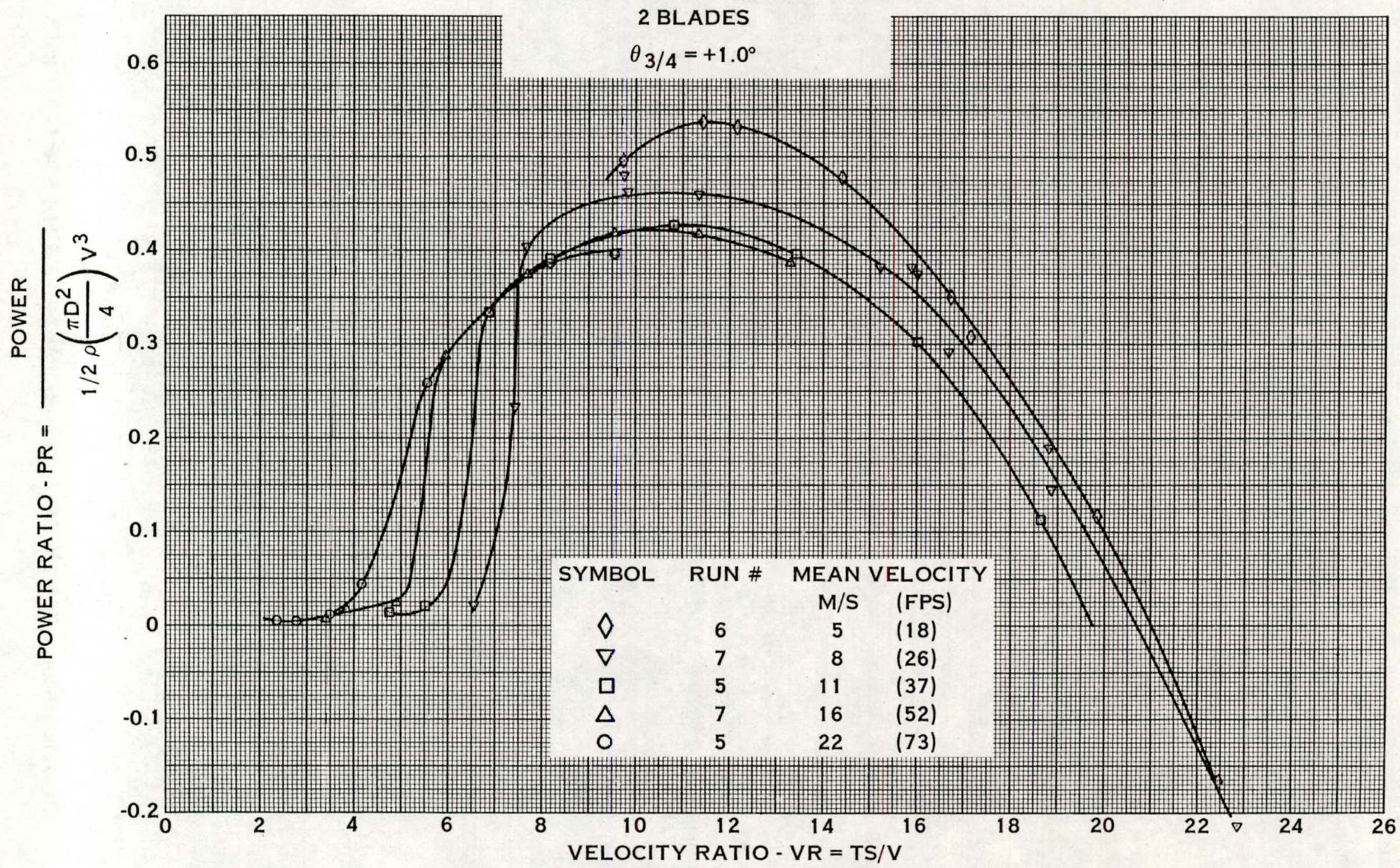

FIGURE 10-6B. EFFECT OF VELOCITY ON MEASURED POWER RATIO 


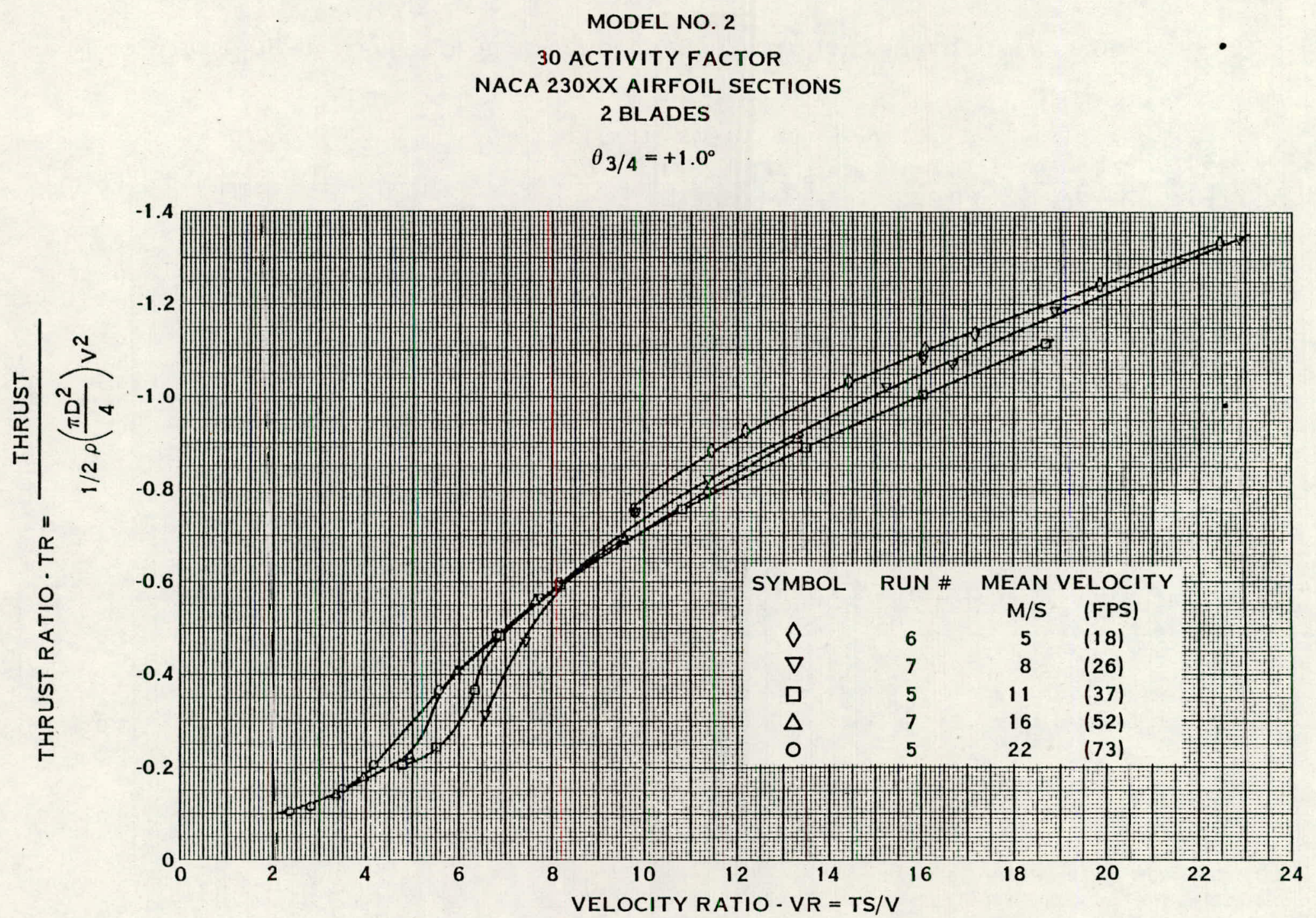

FIGURE 10-6C. EFFECT OF VELOCITY ON MEASURED THRUST RATIO 
MODEL NO. 2

30 ACTIVITY FACTOR

NACA $230 X X$ AIPFOIL SECTIONS 2 BLADES

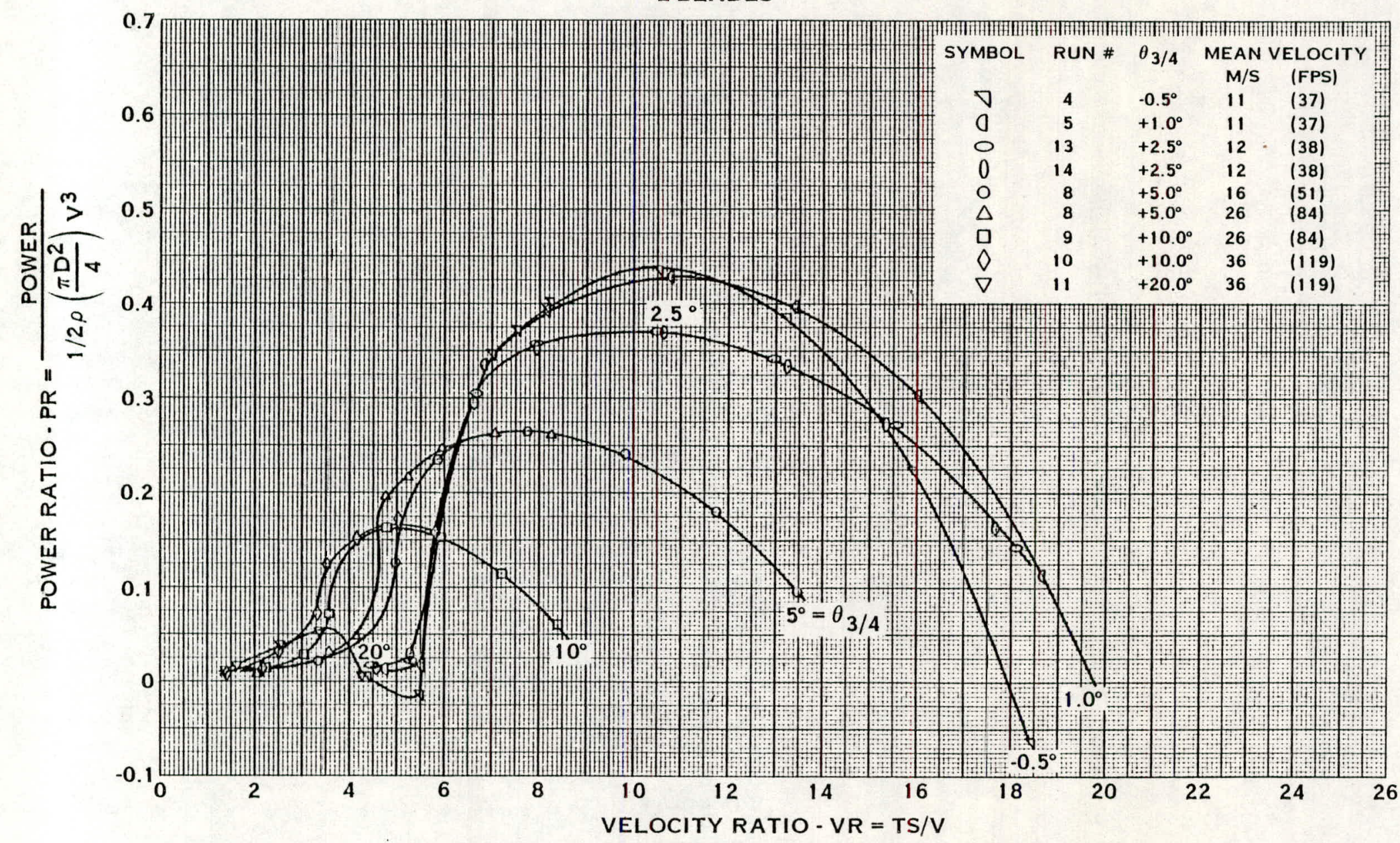

FIGURE 10-6D EFFECT OF BLADE ANGLE VARIATION ON MEASURED POWER RATIO 
MODEL NO. 2

30 ACTIVITY FACTOR

NACA 230XX AIRFOIL SECTION

2 BLADES

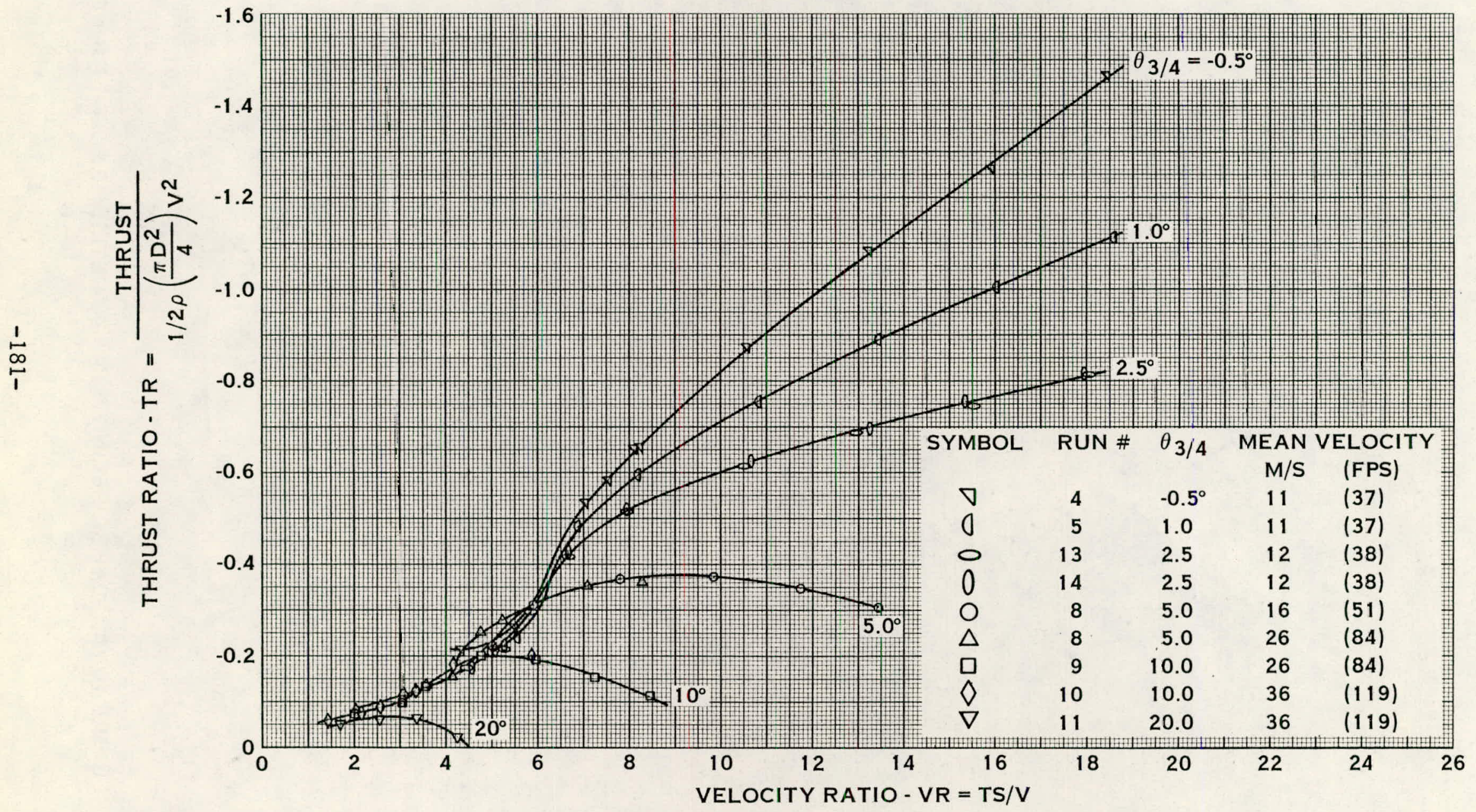

FIGURE 10-6E. EFFECT OF BLADE ANGLE VARIATION ON MEASURED THRUST RATIO 


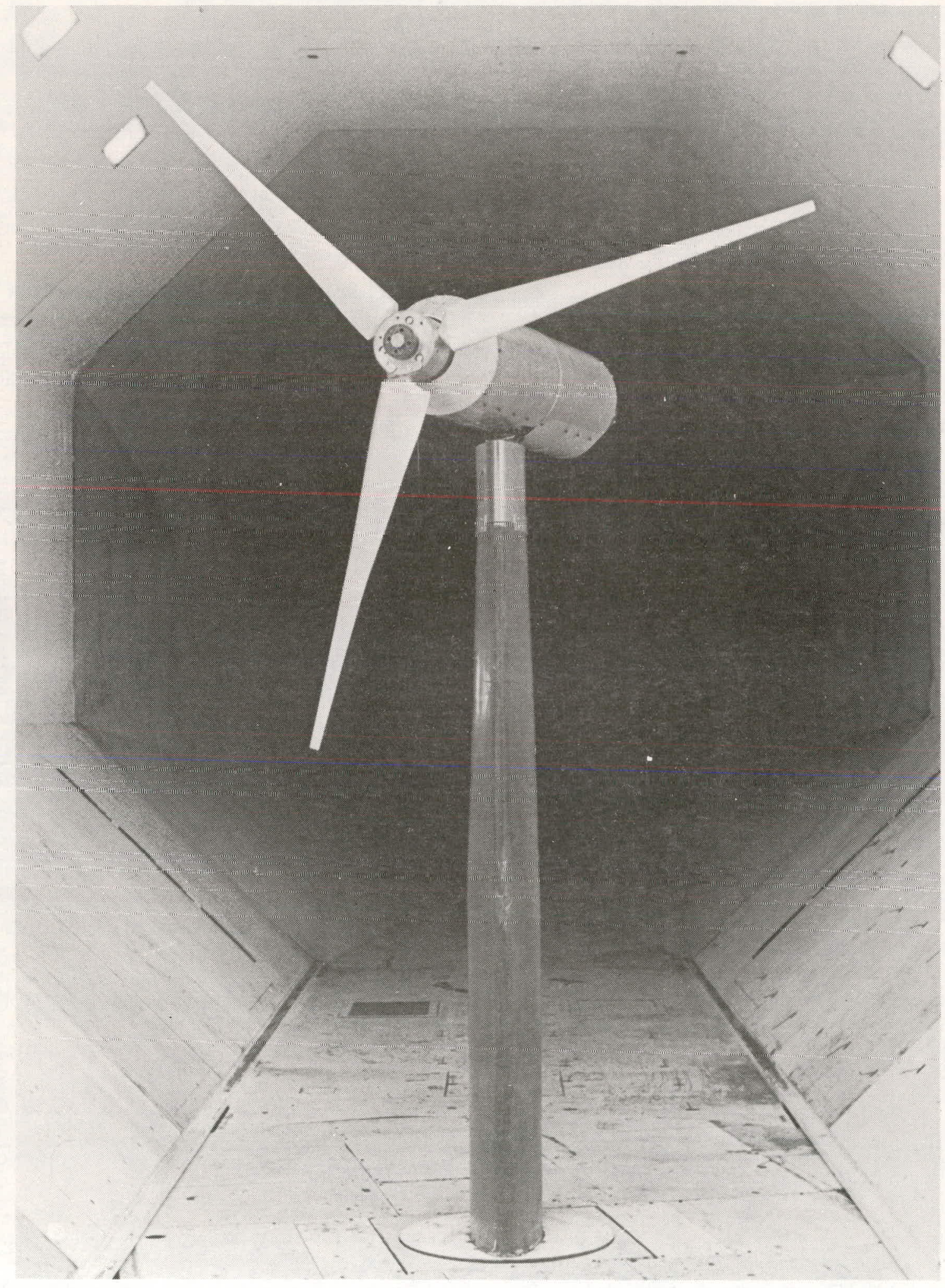

FIGURE 10-7A. 3OAF MODEL NO. 3 IN 5.48 METER (18 FOOT) TUNNEL 


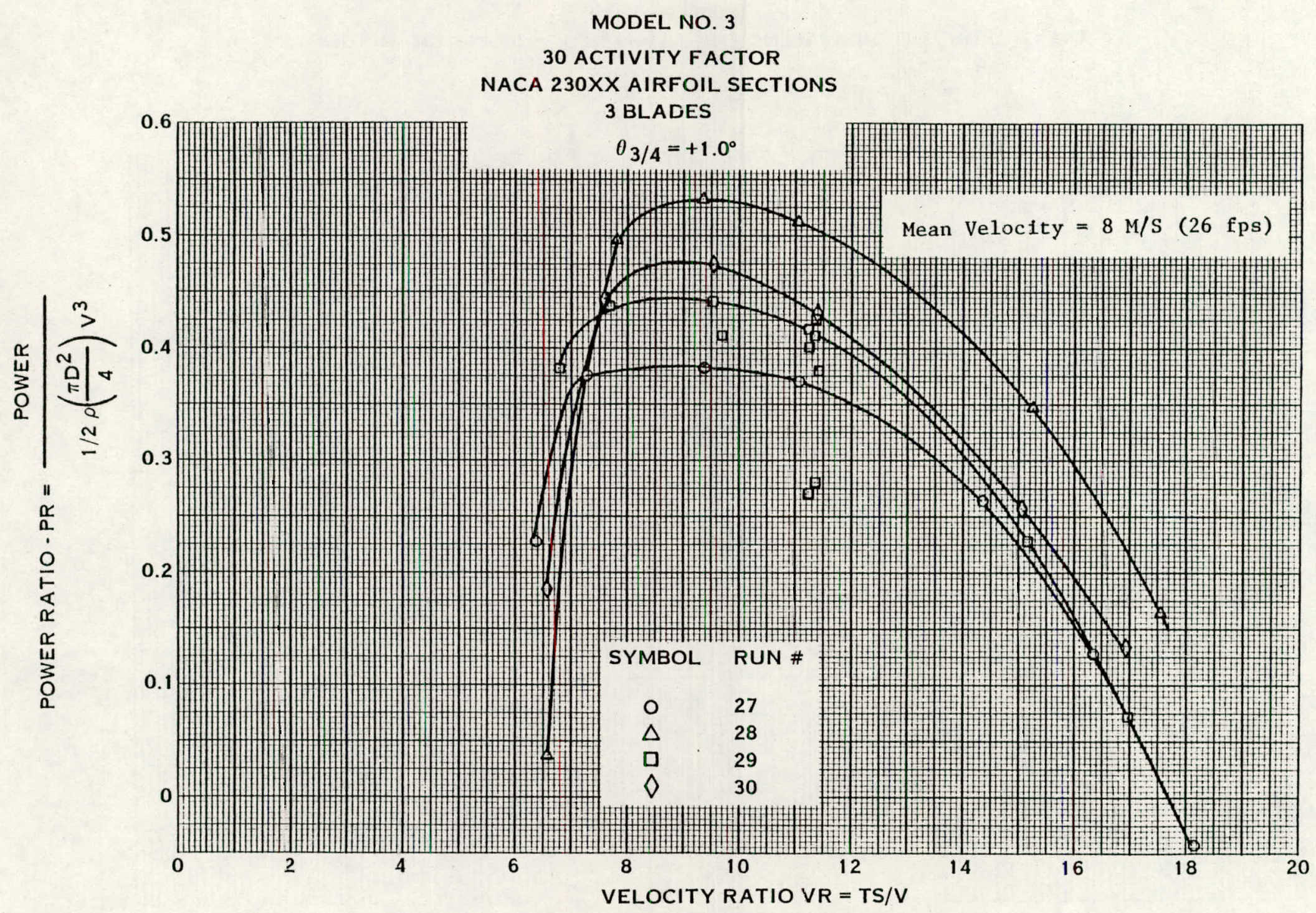

FIGURE 10-7B. EFFECT OF REPEAT RUNS ON MEASURED POWER RATIO 
MODEL NO. 3

30 ACTIVITY FACTOR

NACA 230XX AIRFOIL SECTIONS

3 BLADES

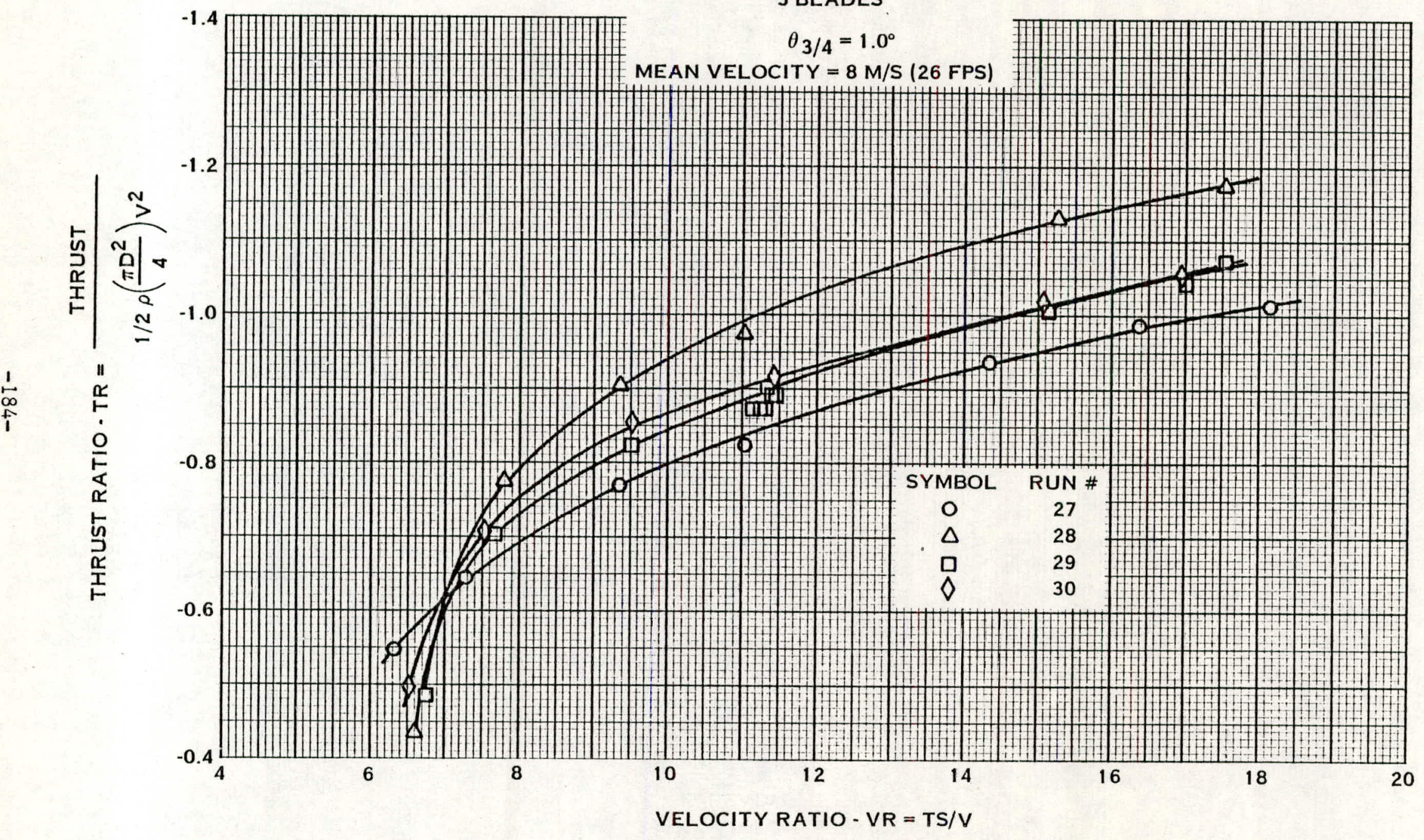

FIGURE 10-7C. EFFECT OF REPEAT RUNS ON MEASURED THRUST RATIO 


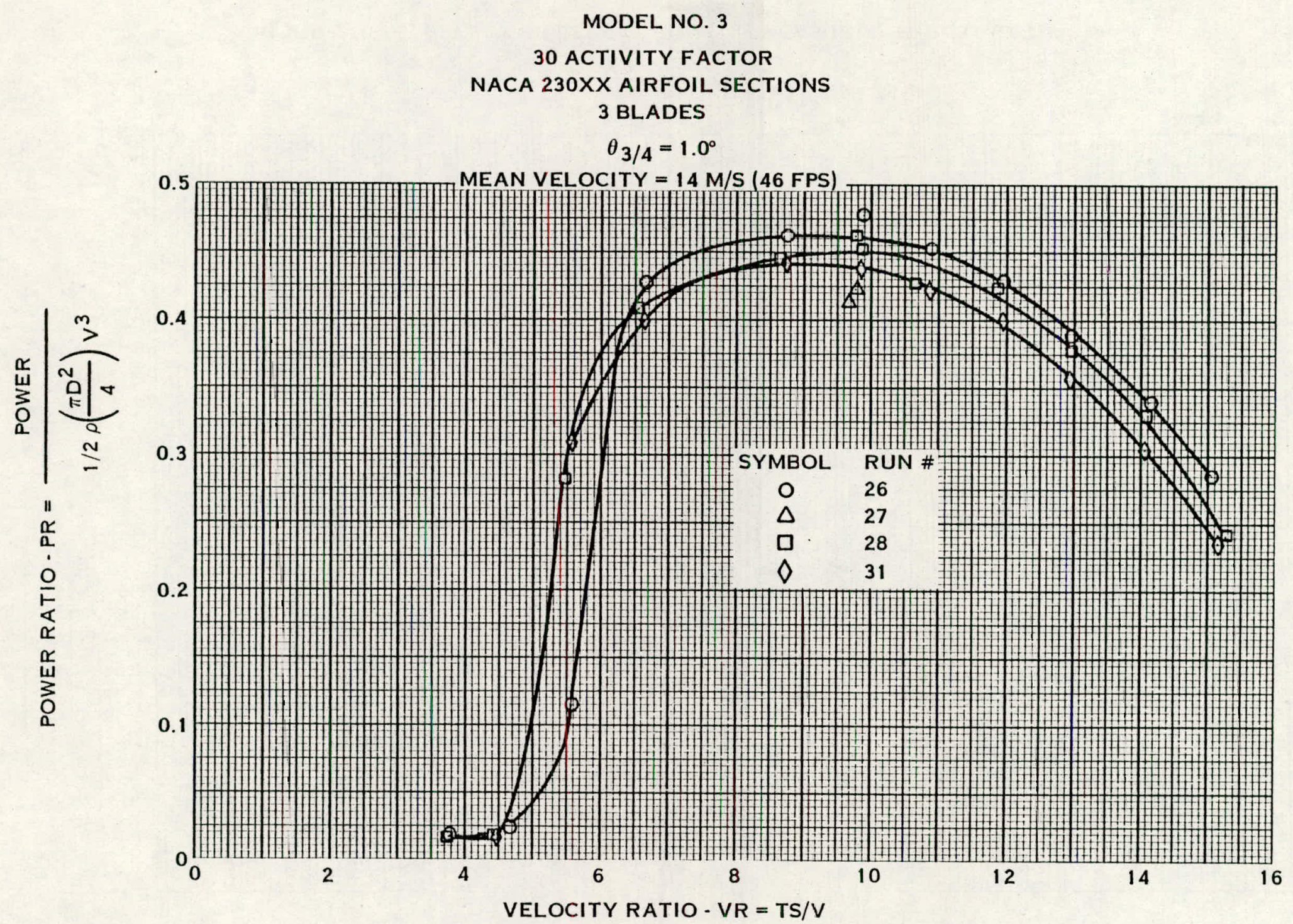

FIGURE 10-7D. EFFECT OF REPEAT RUNS ON MEASURED POWER RATIO 


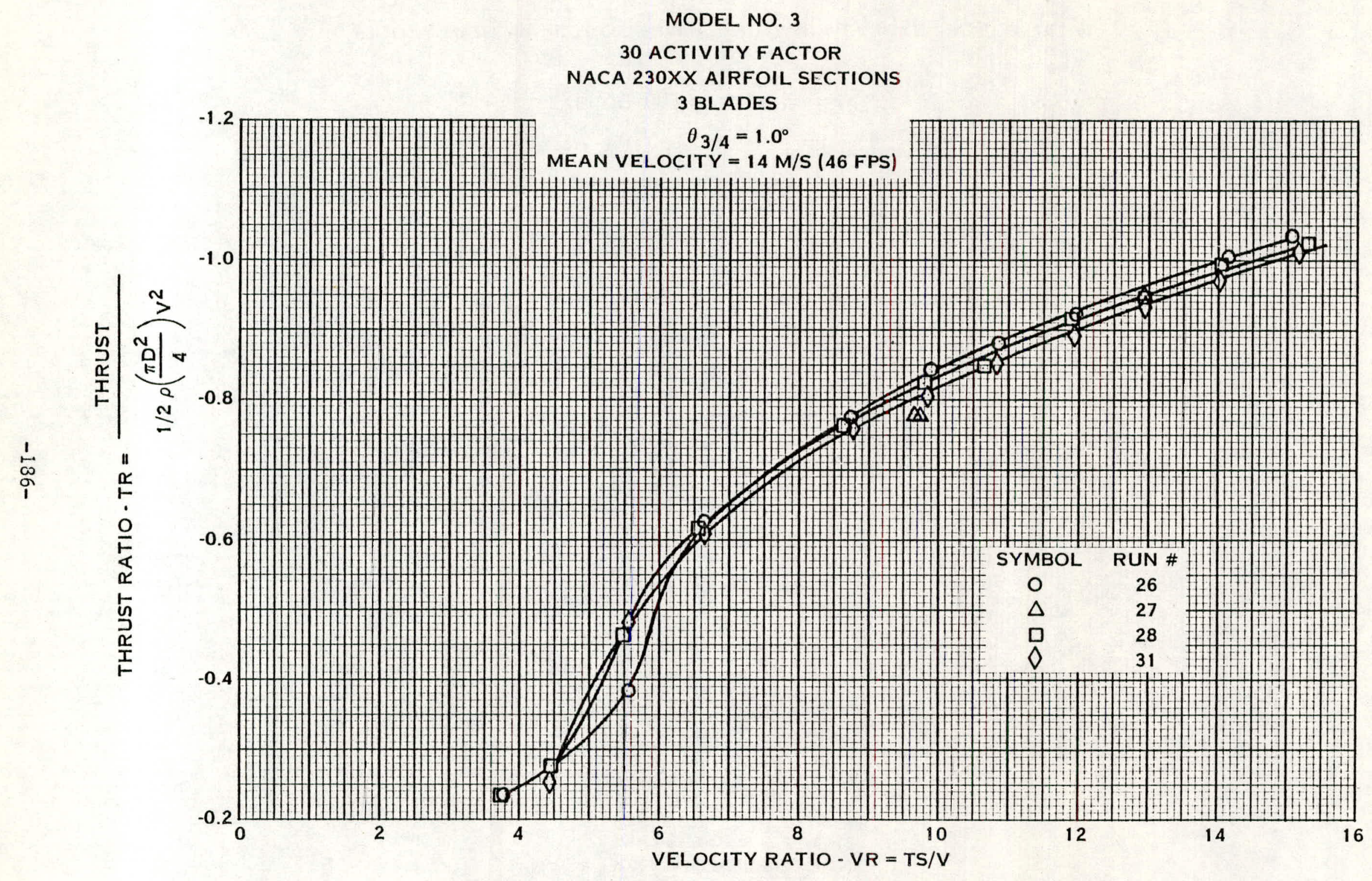

FIGURE 10-7E. EFFECT OF REPEAT RUNS ON MEASURED THRUST RATIO 



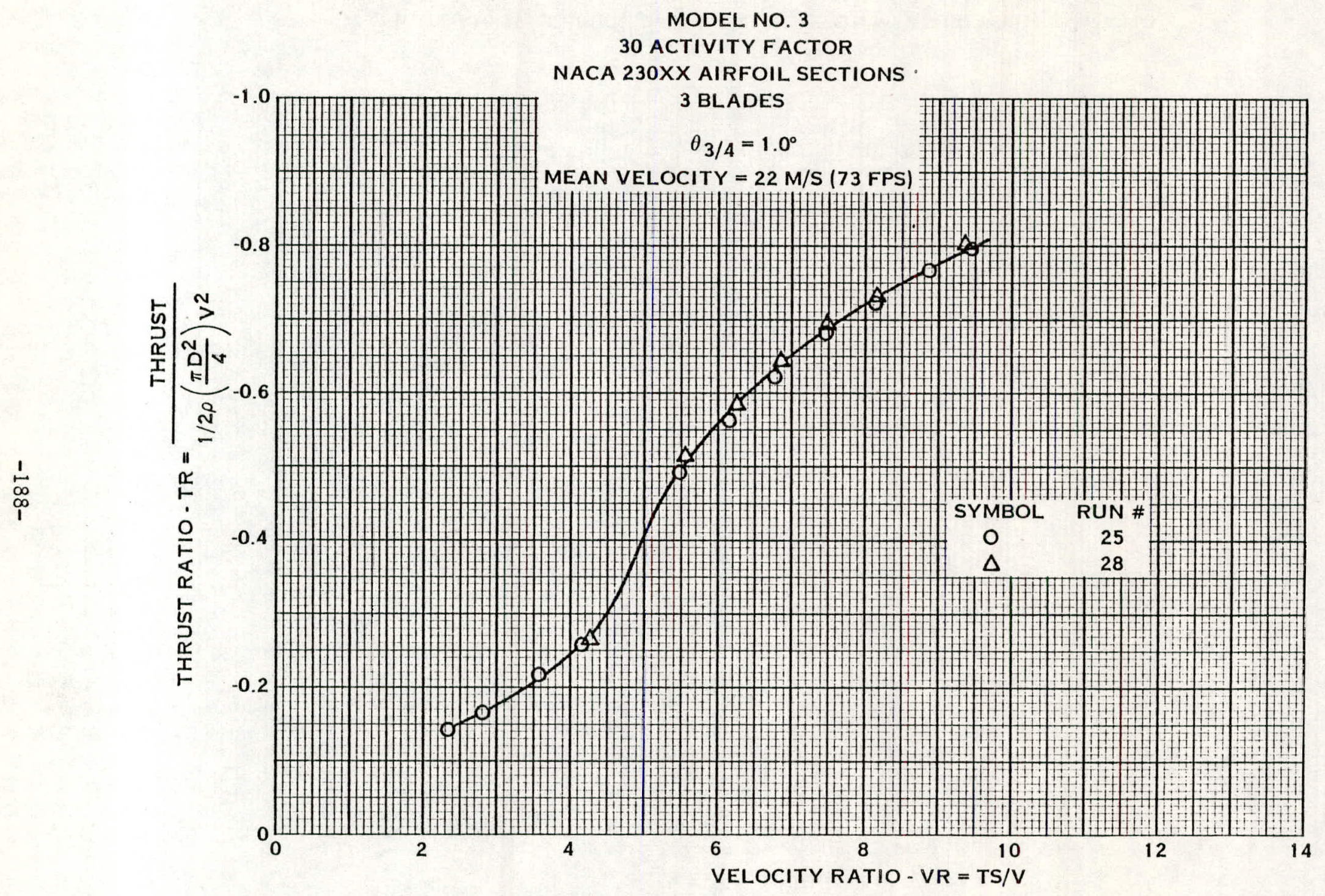

FIGURE 10-7G. EFFECT OF REPEAT RUNS ON MEASURED THRUST RATIO 


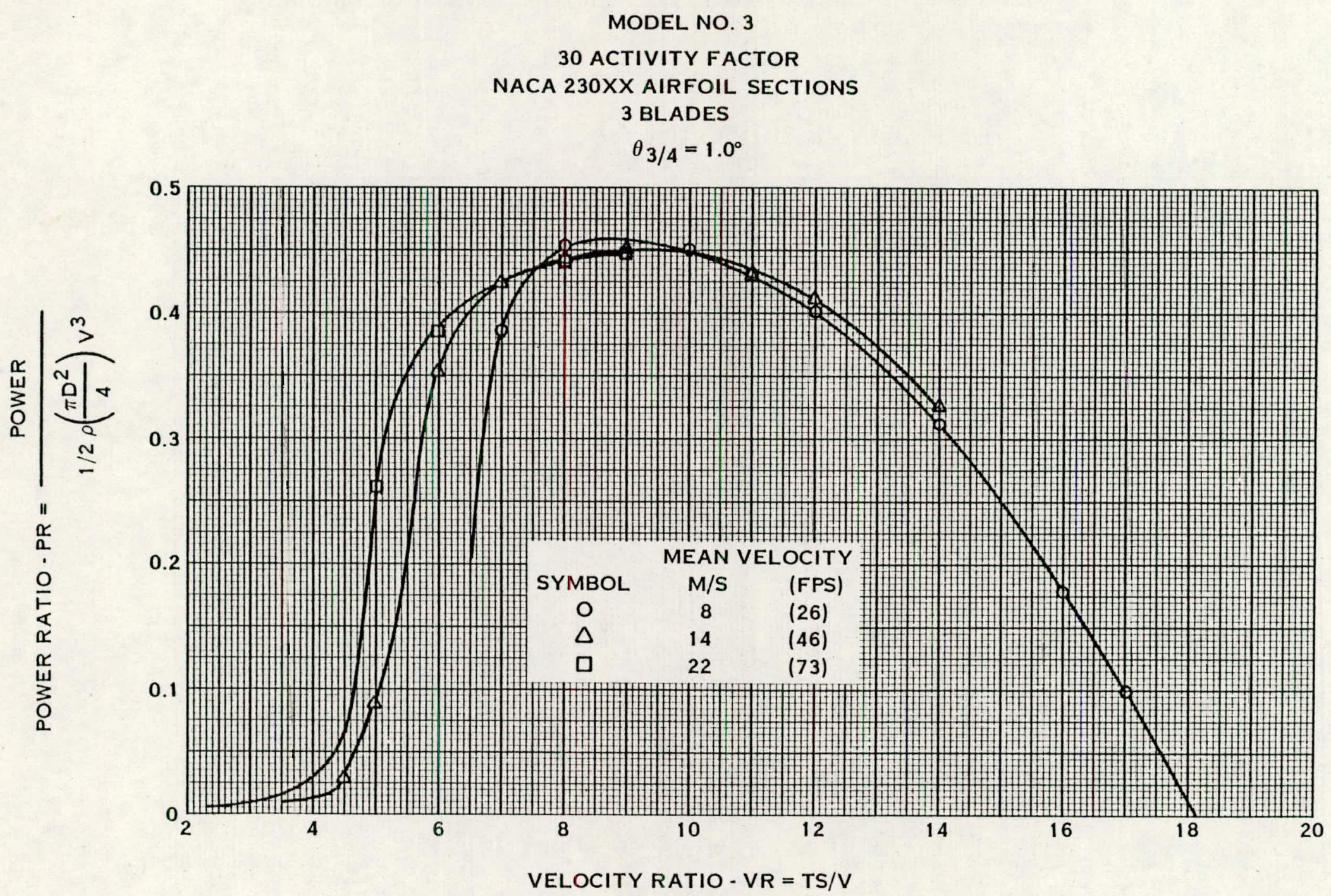

FIGURE 10-7H. EFFECT OF VELOCITY ON MEASURED POWER RATIO 


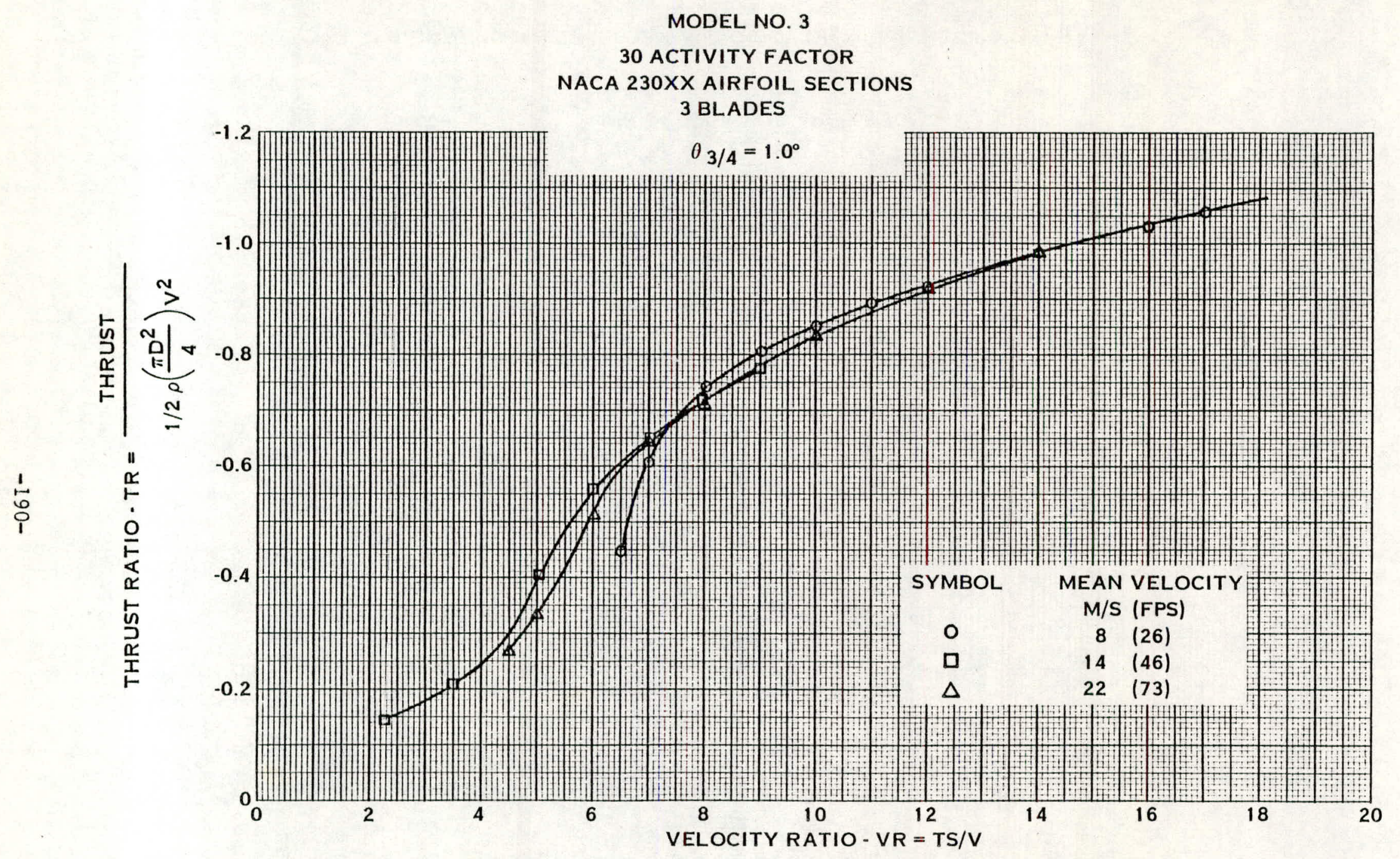

FIGURE 10-7I. EFFECT OF VELOCITY ON MEASURED THRUST RATIO 
MODEL NO. 3

30 ACTIVITY FACTOR

NACA $230 X X$ AIRFOIL SECTIONS

3 BLADES

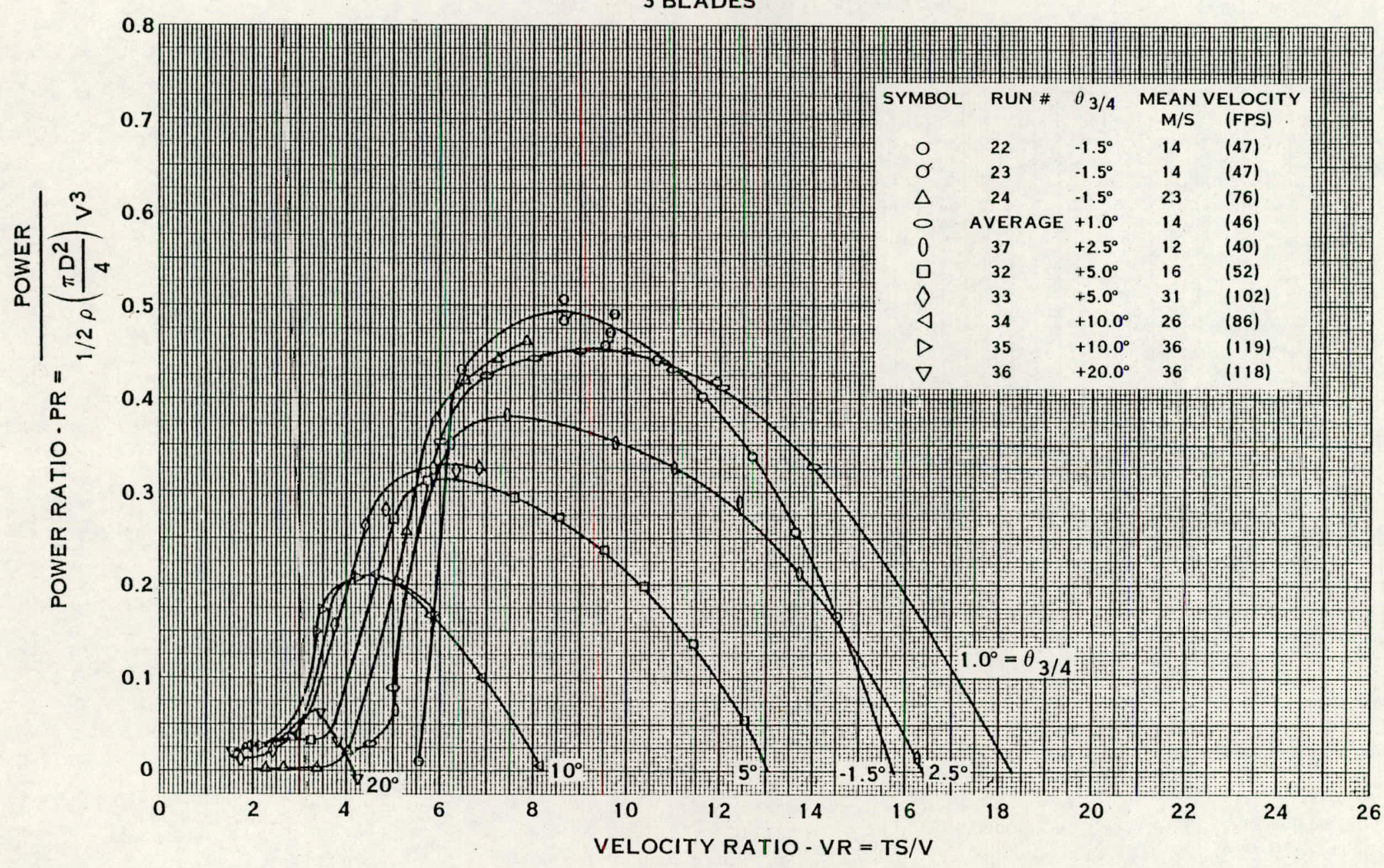

FIGURE 10-7J. EFFECT OF BLADE ANGLE VARIATION ON MEASURED POWER RATIO 
MODEL NO. 3

30 ACTIVITY FACTOR

NACA 230XX AIRFOIL SECTIONS

3 BLADES

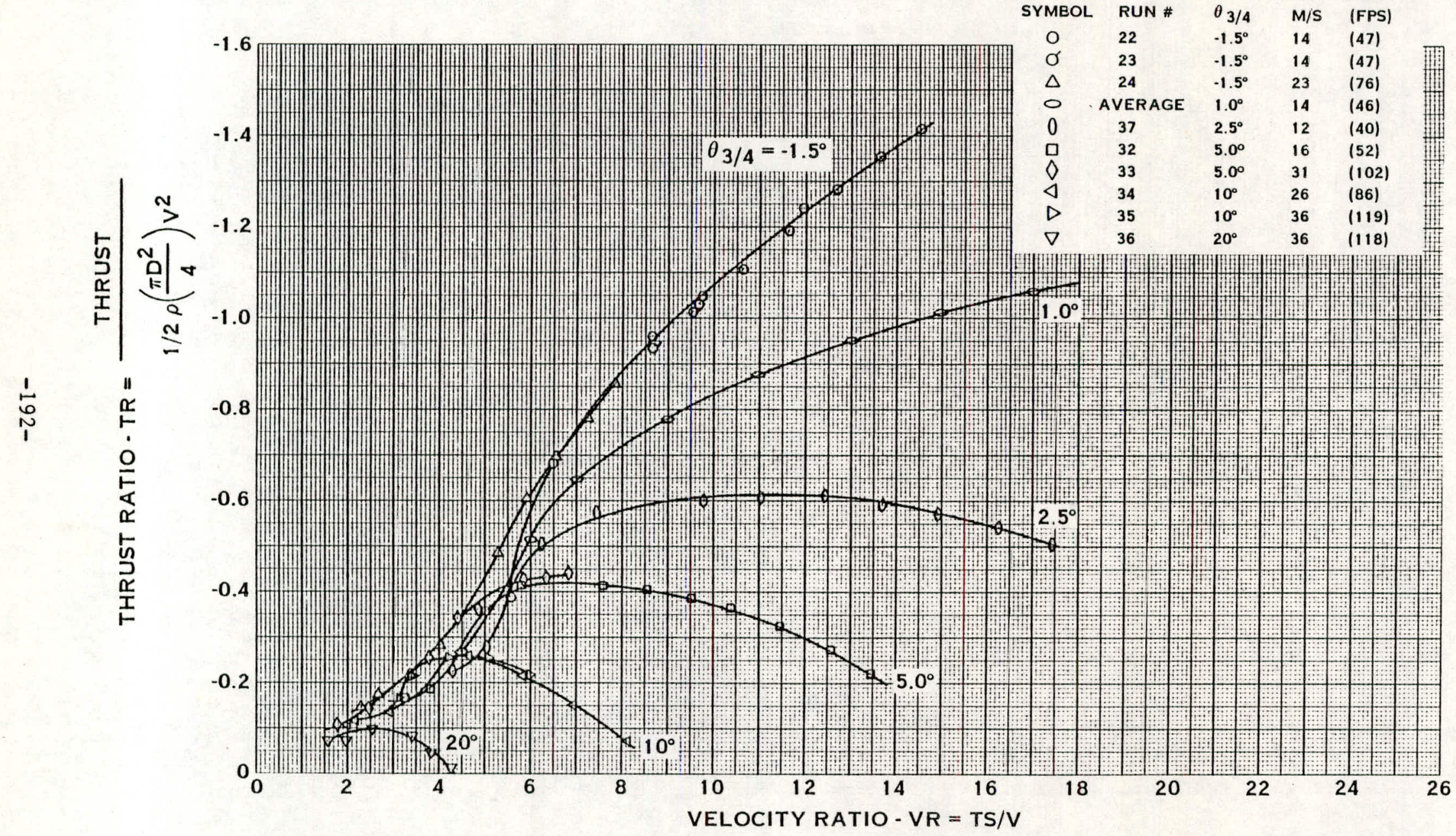

FIGURE 10-7K. EFFECT OF BLADE ANGLE VARIATION ON MEASURED THRUST RATIO 
N.Y.U. TEST DATA

MODEL, A, AF $=97$

$01 / 4=6.3^{\circ}$

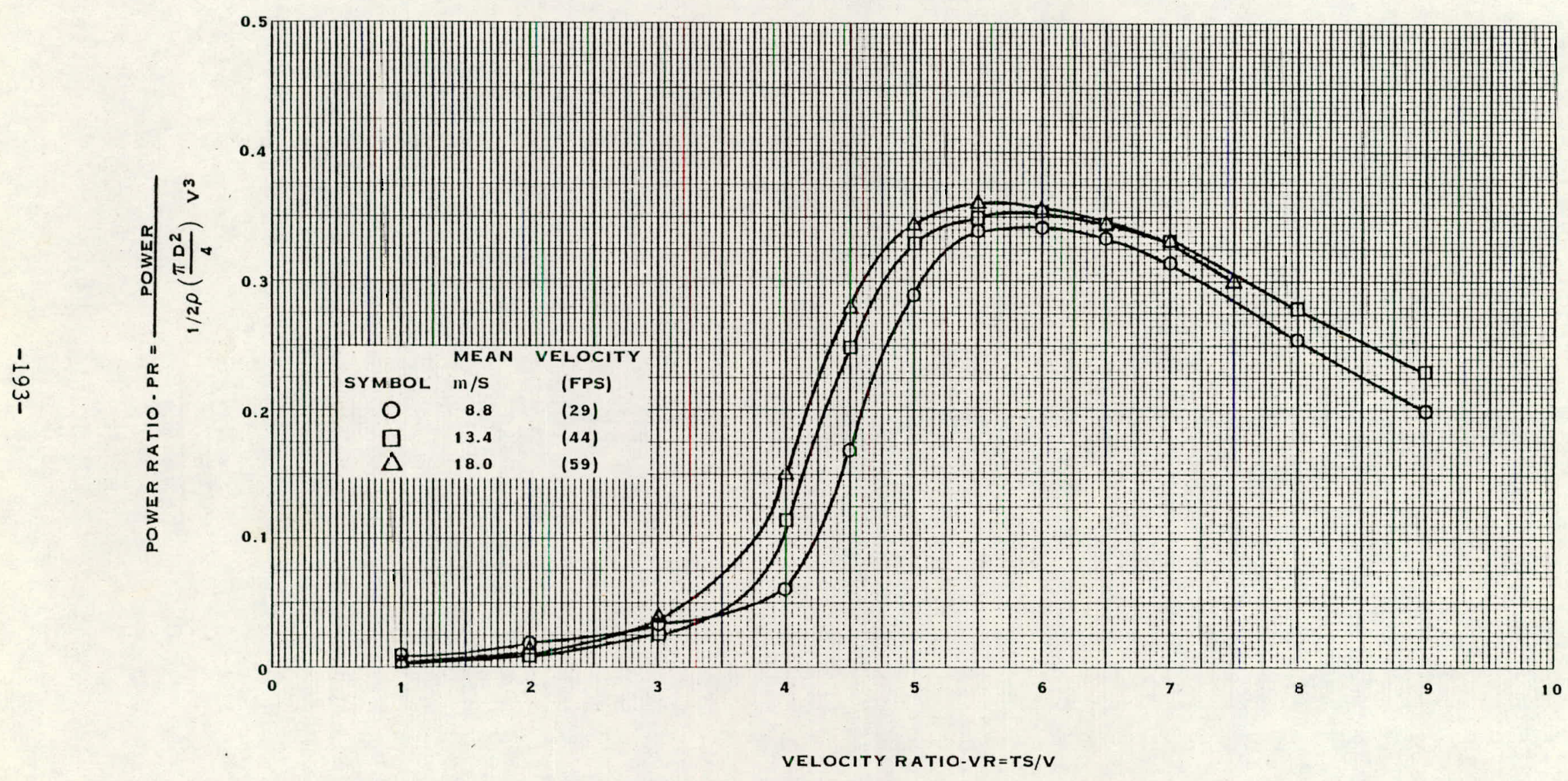

FIGURE 10-8. EFFECT OF VELOCITY ON MEASURED POWER RATIO 
N.Y.U. TEST DATA

MODEL, $C, A F=37$

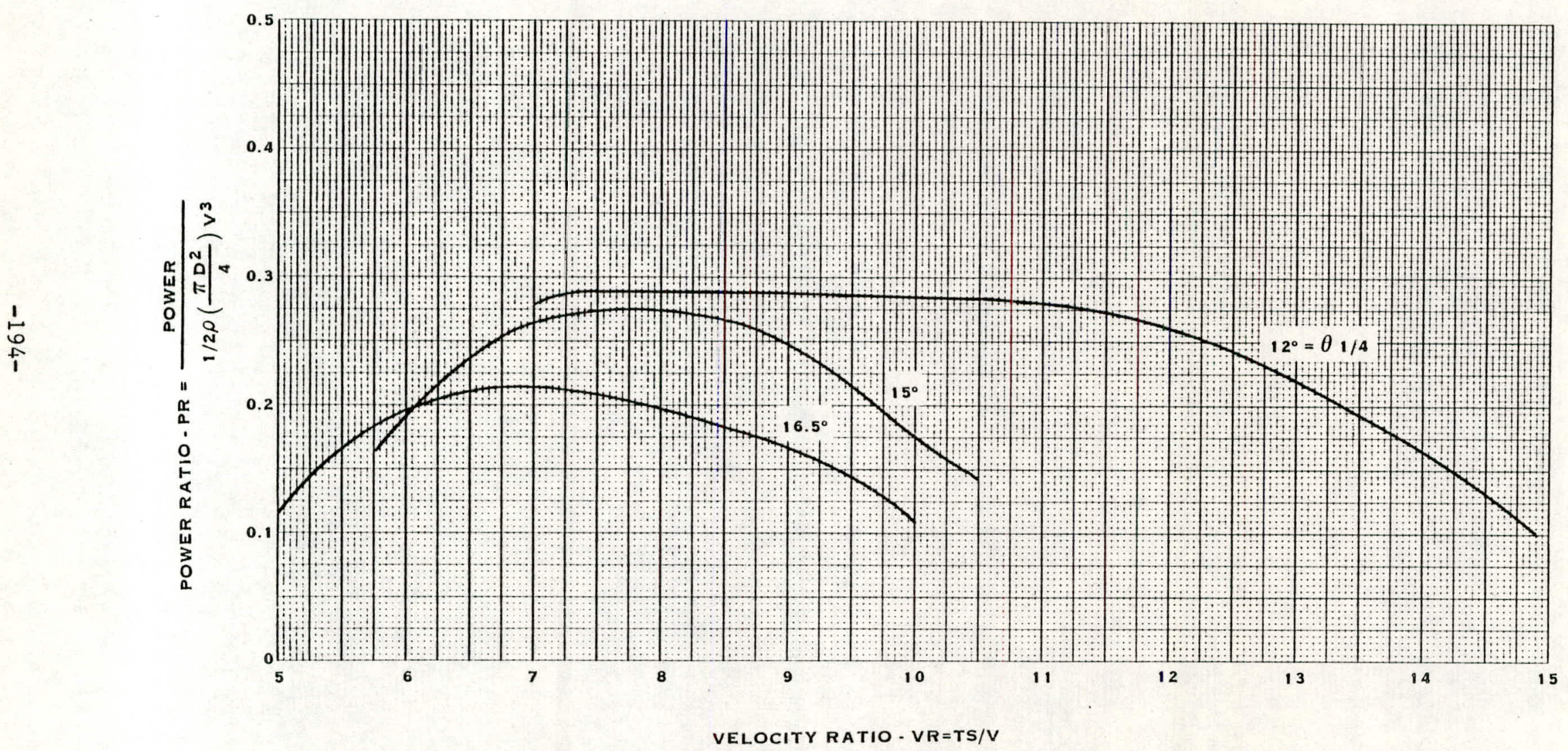

FIGURE 10-9. VARIATION OF MEASURED POWER RATIO 

SULZER TEST DATA

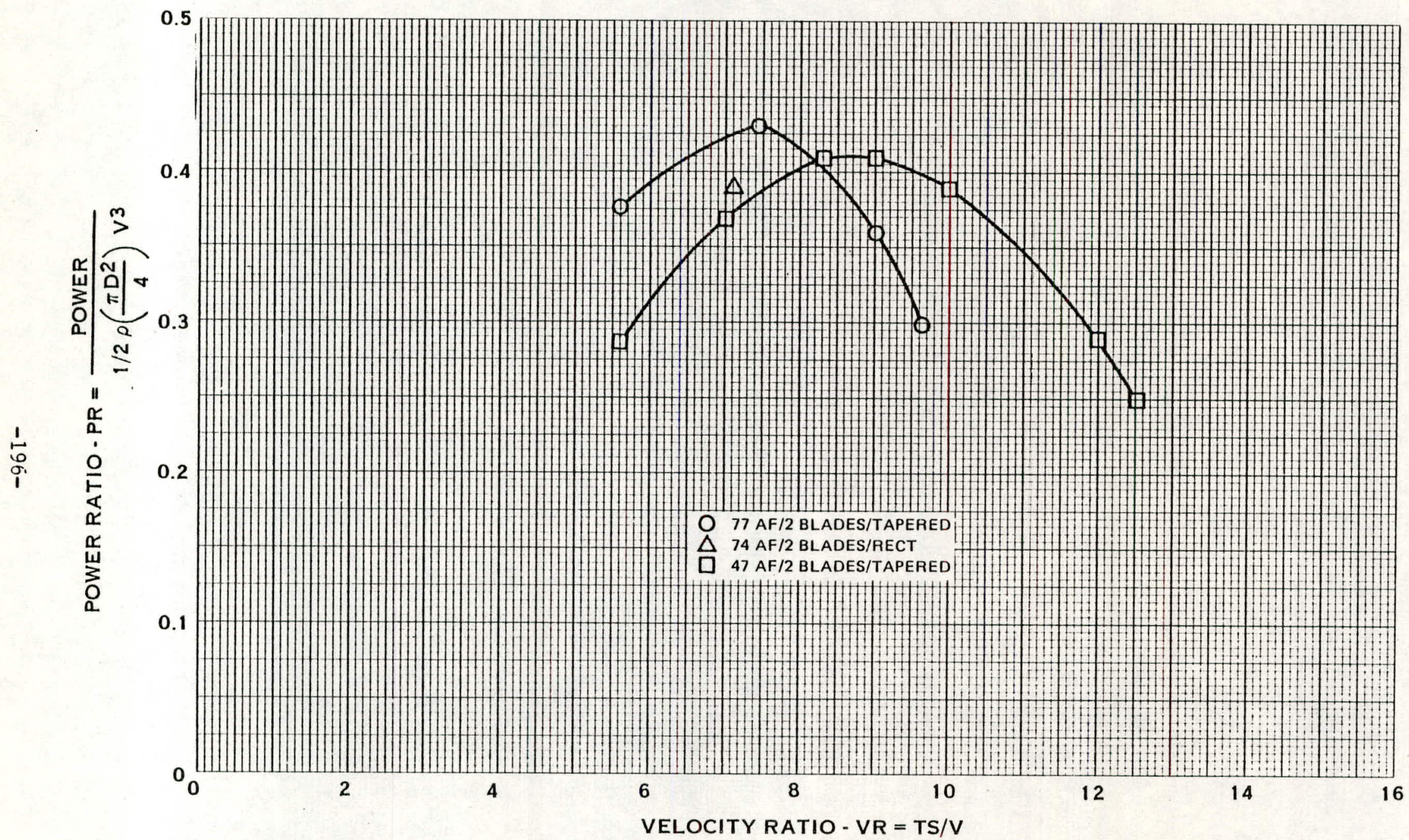

FIGURE 10-11. VARIATION OF POWER RATIO WITH VELOCITY RATIO 



\section{SECTION 11}

\section{DATA ANALYSIS AND EVALUATION}

\subsection{INTRODUCTION}

The predicted performance data for each of the test models were presented in Section 9. The measured performance data for the three models tested in this program and some supplemental test data from two other sources were presented in Section 10. In this section, performance comparisons of calculation and test will be made to evaluate the methodology. Moreover, the effect of Reynolds number, number of blades, and blade solidity as established by the testing will be presented.

\subsection{METHOD VALIDATION}

The calculations of Section 9 are compared with the measured data of Section 10 for the three test configurations as shown in figures 11-1A through 11-3B. The power ratio and thrust ratio variations for a range of blade angles for Model 1 are shown in figures $11-6 \mathrm{~A}$ and $11-6 \mathrm{~B}$, respectively. Since these calculations were made, an internal structure process in the computer program has been improved which effects the calculated performance characteristics at the high velocity ratio range and low blade angles. This revision changed the power ratio curve at high velocity ratios for Model 1 set at $-1^{\circ}$ blade angle as shown in figure 11-1A such that it now more closely follows the test trend. A similar comparison is shown in figures $11-2 \mathrm{~A}$ and $11-2 \mathrm{~B}$ for Model 2. Again, with the revised computer program, calculations show an improved correlation with test data for $-.5^{\circ}$ blade angle in the high velocity ratio range. For Model 3, the comparisons of calculations and test are shown in figures $11-3 A$ and $11-3 B$. The performance for the $1^{\circ}$ blade angle was calculated after the test and includes the revision in the computer program. For all three models, it can be noted that the calculations predict the shapes of the power ratio and thrust ratio test curves quite well. However, the predicted levels are generally lower than the test levels. Moreover, the test data show a sharper drop-off in performance at the low velocity ratio range than predicted by calculations.

To study more carefully the difference between test and calculations, power ratios were plotted versus velocity ratio in figures $11-4$ and 11-5 for the three models at design blade angle. In figure 11-4, the corresponding variations in Reynolds number are shown in the upper set of curves. Since Model 3 was tested at a higher velocity than Model 2, the Reynolds numbers for Model 3 are higher than those for Model 2. The Reynolds numbers for Model 1 are lower than those of Model 2 because the blade chords are smaller. Figure 11-5 compares test and calculations based on a $6 \times 10^{6}$ Reynolds number which is more representative of full scale. From an inspection of these two figures, it is noted that for Models 2 and 3 the calculation based on $6 \times 10^{6}$ Reynolds number more closely match the test data in the vicinity of the peak power ratio than do the calculations based on the model Reynolds number. However, for Model 3 the test performance is more closely predicted by calculation using model Reynolds number. 
An explanation for these observations is that the steep drop-off in peak power ratio may occur at a significantly lower Reynolds number than predicted in figure 9-9C. In the low velocity ratio range where the angles-of-attack on the wind turbine blades approach stall, neither model or full scale Reynolds number calculations match the test data. Thus the airfoil data generalization incorporated in the computer program may not properly represent the entire test Reynolds number range.

In view of the foregoing, it is appropriate to discuss the methodology and the airfoil generalizations as the basis for better understanding the discrepancies between calculated and measured performance.

\subsubsection{Methodology}

The calculations for the test models were made using the Goldstein method together with a generalization of 2-dimensional airfoil data spanning a large Reynolds number range as discussed in Section 3. A comparison was made of calculations based on this method with calculation based on the wind turbine computer program (ref. 22) developed at Oregon State University as part of a study supported by the National Science Foundation, Research Applied to National Needs (RANN), under Grant No. GI-41840. This strip analysis method utilizes the same airfoil section, NACA 23018, all along the blade. Thus, although blade chord and twist are included in the computation, the thickness ratio is constant along the blade. Calculations were made using this program. For a direct comparison, the Hamilton Standard calculations were rerun using Hamilton Standard airfoil data for a NACA 23018 rough section for $6 \times 10^{6}$ Reynolds number. Therefore, both programs were run using comparable airfoil data. Both sets of calculations are shown in figure 11-6. It can be seen that both calculations compare well, thus indicating a consistency between the two programs.

As the last phase of the test program, it was planned to obtain flow visualization data for assessing the Prescribed Wake and the Rotor Wake Geometry programs described in Section 3. Due to the termination of the test program, these data were not obtained; however, to assess the effect of a wind turbine wake on performance, the computed free wake data described in Section 3.6 was used in the Prescribed Wake Program to compute one performance point for Model 2. The calculation was made using the airfoil data for model Reynolds number so that a direct comparison could be made with the predicted model performance. From figure 11-6, it can be seen that the calculation hased on this wake definition would indicate a higher performance level than that predicted by Goldstein and could account for one-third of the difference between calculation and test. It is apparent that sufficient flow visualization data of the wake should be obtained to permit a thorough evaluation of the importance of wind turbine wake characteristics in predicting wind turbine performance.

\subsubsection{Airfoil Data Generalization}

The 2-dimensional airfoil data generalization included in the computational procedure was dereived from published, 2-dimensional test data on the NACA $230 X X$ airfoil series. The test airfoils include a thickness ratio range from 12 through 24 percent. The testing covered 
a Reynolds number range from 3 to $9 \times 10^{6}$ and an angle-of-attack range of $\pm 20^{\circ}$. In addition only limited data for these airfoils have been published for thinner sections and for the low Reynolds number range of this test program; i.e. $75 \times 10^{5}-1.2 \times 10^{6}$. Therefore, in order to derive the airfoil data generalization for the design and performance prediction of both full scale and model wind turbine, it was necessary to extrapolate the published airfoil data. Through the extrapolation process, the 2-dimensional airfoil data has been extended to cover a family of airfoils from 6 to 40 percent thick, operating over an angle-of-attack range of $\pm 20^{\circ}$ and a Reynolds number range from $75 \times 10^{6}$ through $9 \times 10^{6}$. Thus this airfoil data generalization is suitable for calculating the performance of the test models of this program as well as full scale wind turbines.

However, it should be pointed out that a study of the airfoil data for the thick sections, undertaken after the design of the test wind turbine models, indicates that the extrapolated drag data in the generalization may be pessimistic.

Thus, the performance predicted for the inner half of the blade radius, where the airfoil sections are above $25 \%$ thick, may be unrealistically low. To evaluate the importance of the inner half of the blade, computations were made for the Model 2 assuming a maximum thickness of $25 \%$. The calculations were plotted on figure 11-7. At peak power ratio, it can be seen that the calculations predict a $5 \%$ drop in performance due to the thick sections, thus indicating that the drop-off shown should be somewhat less and in the direction to lessen the discrepancy between calculations and test.

As noted, the airfoil data generalization is based primarily on airfoil test data most of which are for 3 to $6 \times 10^{6}$ Reynolds number. Therefore, the extension of the airfoil generalization to the low Reynolds numbers corresponding to those encountered in the test was based on very limited data. Calculations based on $6 \times 10^{6}$ Reynolds number more closely match the test data at peak power ratio as shown in figure 11-7. This would indicate that the increase in drag due to R.N. may not be as severe as the generalizations predict.

As was stated previously, the sharp drop-off in the test performance at the low V.R. range may be attributed to an earlier stall due to low Reynolds number. In the high angle-of-attack range, the extrapolation of the drag generalization is particularly uncertain. The present drag generalization consists of a minimum drag which is a function of thickness ratio and Reynolds number, a drag due to lift which is a function of lift coefficient and maximum lift coefficient for the section (also function of R.N.) and a drag extrapolation for the high angle-of-attack range. An attempt at improving the drag revision was made by revising the latter portion of the generalization to be a function of stall angle for the R.N. As shown in figure 11-12, the revision resulted in a trend similar to the test trends.

Thus, it can be concluded that a large portion of the discrepancy between calculations and test could be attributed to the inadequacy of the airfoil generalization in the low R.N. range corresponding to these tests. 


\subsection{EFFECT OF PRIME PARAMETERS}

From the test data of Section 10, plots of the pertinent experimental data were made to show the effects on performance of Reynolds number (fig. 11-8) and the geometric parameters of number of blades (fig. 11-9) and activity factor (fig. $11-10$ and 11-11). The experimental trends with these parameters and their comparison with predicted trends are discussed in the following text.

\subsubsection{Effect of Reynolds Number}

Figure 11-8 presents a plot of peak power ratios versus the corresponding Reynolds number at $3 / 4$ radius for the 2 blade Hamilton Standard test models and for the 2 blade supplemental models of references 20 and 21 . As can be seen in this figure, the measured data verify the predicted sharp drop-off in the low Reynolds number range. However, this drop-off occurs at $.35 \times 10^{6}$ Reynolds number which is significantly below the predicted value of approximately $1.0 \times 10^{6}$ Reynolds number shown in figure 9-9C. However, the test data do not confirm the increasing peak power ratio level with increasing activity factor at a given Reynolds number predicted in figure 9-9C. This effect may have been obscured since the test data are from various sources which introduce different measurement accuracies among the test facilities as well as differences in model size, finish and in the details of the shape characteristics. Unfortunately, Models 5 and 6 with the $60 \mathrm{AF}$ blades were not tested. The higher Reynolds number level of these models would have provided key data on similar models from the same test facility. Thus a more complete and consistent story on the Reynolds number effect could have been achieved.

In connection with the problem of dealing with low Reynolds number effects when testing scale models in the wind tunnel, it is recalled that a cursory attempt at flow transition control was made by applying an arbitrary density of grit to the leading edge radius on Model 1 ( 2 blade/ $15 \mathrm{AF}$ ). As previously reported, this was done in an attempt to investigate the abrupt fall-off in power ratio at low velocity ratios during the initial testing of this model. Although the performance level was reduced significantly over the higher velocity ratio range, the abrupt fall-off in power ratio was alleviated in the low velocity ratio range where angles-of-attack on the blades approach stall and the Reynolds number is reduced. Thus it would appear that with careful control of the grit size and distribution on the model blades, the potential exists in simulating full scale Reynolds number performance on the model wind turbines included in this test program reported herein.

\subsubsection{Effect of Geometric Variables}

The measured number of blade variation is shown in figures $11-9$ for 1,2 and 3-bladed models. The 30 activity factor model was tested as a 2 and a 3-bladed configuration. The data of reference 21 includes 47 and 77 activity factor models, tested both as 1 and 2-blade configurations. From an inspection of this figure it is noted that the peak power ratio increases at a decreasing ratio as number of blades is increased. For the $30 \mathrm{AF}$ model of this program, the comparison of predicted and measurements peak performance are shown in the following table: 
Peak Performances

PREDICTED (Fig. 9-11)

$\begin{array}{ccccccc}\text { \# Blades } & \text { V.R. } & \text { P.R. } & \text { CHANGE } & \text { V.R。 } & \text { P.R. } & \text { CHANGE } \\ 3 & 9.0 & .410 & & 9.0 & .45 & \\ 2 & 11.0 & .385 & 6 \% & 11.0 & .425 & 6 \% \\ 1 & 16.0 & .325 & 19 \% & & - & -\end{array}$

It is shown in the above table that for both 3 and 2 blades, the velocity ratio corresponding to peak power ratio was well defined. Moreover, although the predicted peak power ratio level was lower than measured, the percentage change due to increase in number of blades was predicted. This comparison cannot be made with the 1 blade model since it was not tested prior to the accident. Although a similar comparison cannot be made for the other 47 and $77 \mathrm{AF}$ configurations from reference 21, it is of interest to note that there is a measured 12 percent reduction in performance between 2 and 1 blades for the $47 \mathrm{AF}$ model and for the $77 \mathrm{AF}$ a measured reduction of 21 percent which spans the 19 percent reduction predicted for the $30 \mathrm{AF}$ model. Moreover the peak power ratio levels for both the 1 and 2 blade models are within experimental error.

The measured activity factor variation is shown on figure 11-10 for the 2 blade configurations and on figure 11-9 for the 1 blade configurations. To compare the measured data with the predicted data of figure 9-11, peak power ratio and the corresponding velocity ratios for the 2 blade models are plotted on figure 11-11. From an inspection of figure 11-11, it can be seen that the trend of peak power ratio variation is well predicted. The $37 \mathrm{AF}$ model is probably out of line because, as noted previously, it had different twist distributions on each blade and moreover was tested at low Reynolds number. The velocity ratio corresponding to the peak power ratio are well predicted as shown in figure 11-11. For the two 1 blade models, the measured performance (fig. 11-9) is essentially the same and thus demonstrates the same trend as the 2 blade models.

Included in the testing in reference 21, were two 2 blade configurations of approximately 75 activity factor. One configuration is a near optimum design and the other configuration has a rectangular planform with no twist. From figure 10-11, it can be shown that the performance is reduced 9 percent by these deviations from the optimum configuration. These same deviations were investigated for the 1 blade configurations with 46 activity factor and correspondingly there was a 28 percent reduction (fig. 10-12) in performance. It should be recalled that these same effects were individually analytically investigated for a 2 blade/30 AF optimum configuration predicting a total reduction of 21 percent (Table 4-1). Moreover, the study of reference 5 indicated that the performance penalty in deviating from the optimum planform and optimum twist are much reduced as the activity factor is increased from 30 to 60 which is substantiated by the 2 blade/75 AF data. .This effect of deviation from the optimum design can also be related to a velocity ratio; i.e. this effect is increased with increasing velocity ratio. This would then account for the higher reduction in the peak power ratio for the 1 blade/46 AF models because then peak velocity ratio is higher than that for the 2 blade/ $30 \mathrm{AF}$. 
It should be pointed out that the models of reference 21 incorporated the GA(W)-1 airfoil section which could have had some effect on the comparisons discussed above since the models of this program incorporated the NACA 230XX section. However, because of the probable differences in measurement accuracies between the two test facilities as well as model differences, no effect of airfoil section on performance could be identified. Again the importance of conducting parametric investigations on similar models and in the same test facility must be emphasized. Thus the planned tests on the two 2 blade/60 AF models with NACA $230 \mathrm{XX}$ and $44 \mathrm{XX}$ airfoils, respectively, would have provided valuable information on the effect of airfoil section on wind turbine performance.

\subsection{SUMMARY}

From the foregoing discussion, it was shown that the test data generally substantiate the variations in geometric parameters and Reynolds number predicted by the existing aerodynamic methodology. However, the predicted performance levels are lower than the test levels. In this connection, it was noted in the discussion that possible methodology and airfoil data generalization deficiencies could have contributed to the lower computed levels. Moreover, the wind tunnel turbulence level is in the order of $2 \%$, which may be sufficient to produce a turbulent boundary layer on the leading edge of the model blades indicative of full-scale Reynolds number. Thus, the full-scale test program, as originally planned, is required to better define the effect of geometric and aerodynamic parameters on wind turbine performance and to establish the need for more refined methodology. Additional efforts in this connedtion are discussed in Section 12, Identification of Aerodynamic Technology Needs. 
MODEL NO. 1

15 ACTIVITY FACTOR

NACA 230XX AIRFOIL SECTIONS 2 BLADES

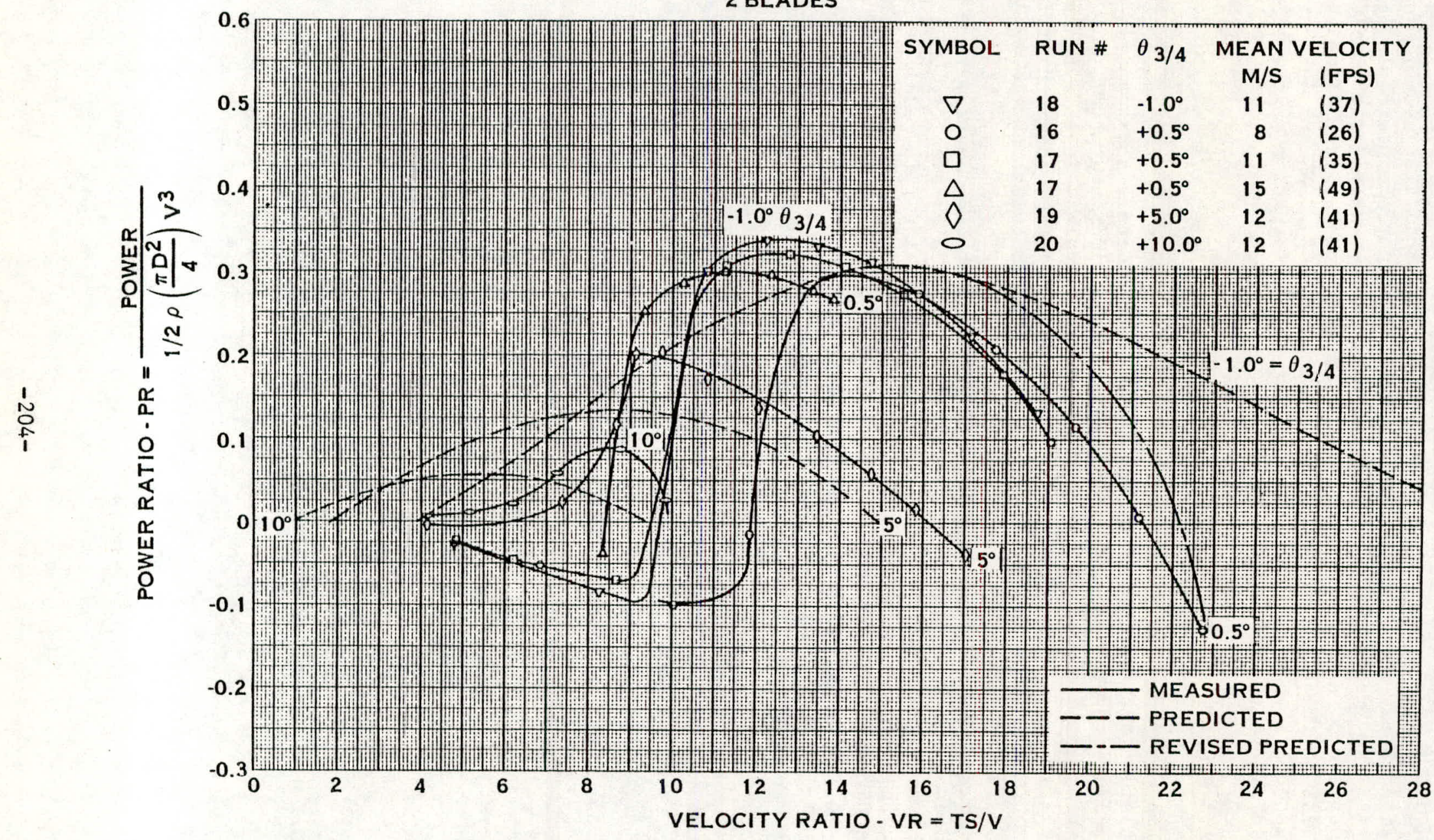

FIGURE 11-1A COMPARISON BETWEEN MEASURED AND PREDICTED POWER RATIO 



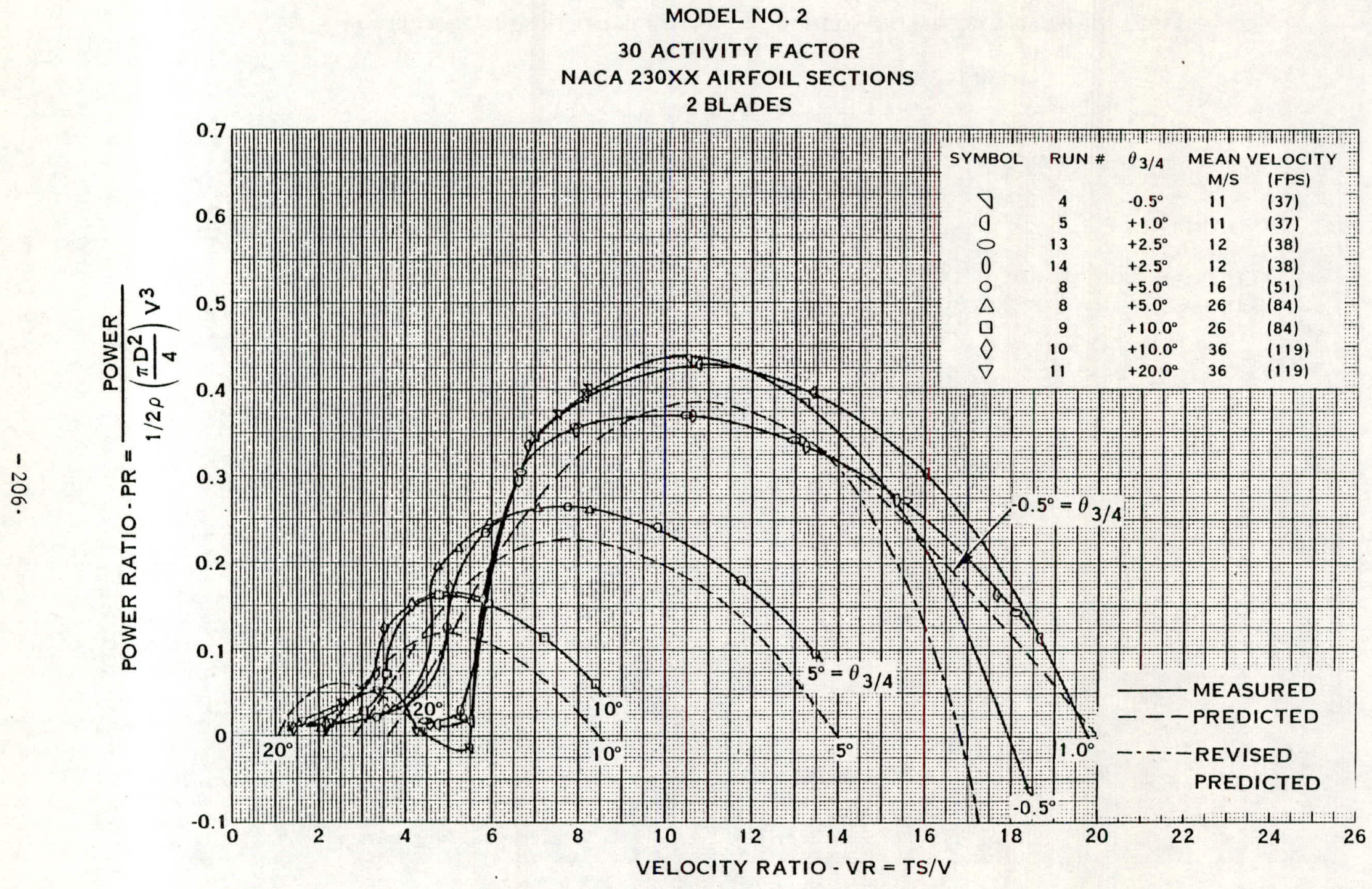

FIGURE 11-2A. COMPARISON BETWEEN MEASURED AND PREDICTED POWER RATIO 
MODEL NO. 2

30 ACTIVITY FACTOR

NACA 230XX AIRFOIL SECTION 2 BLADES

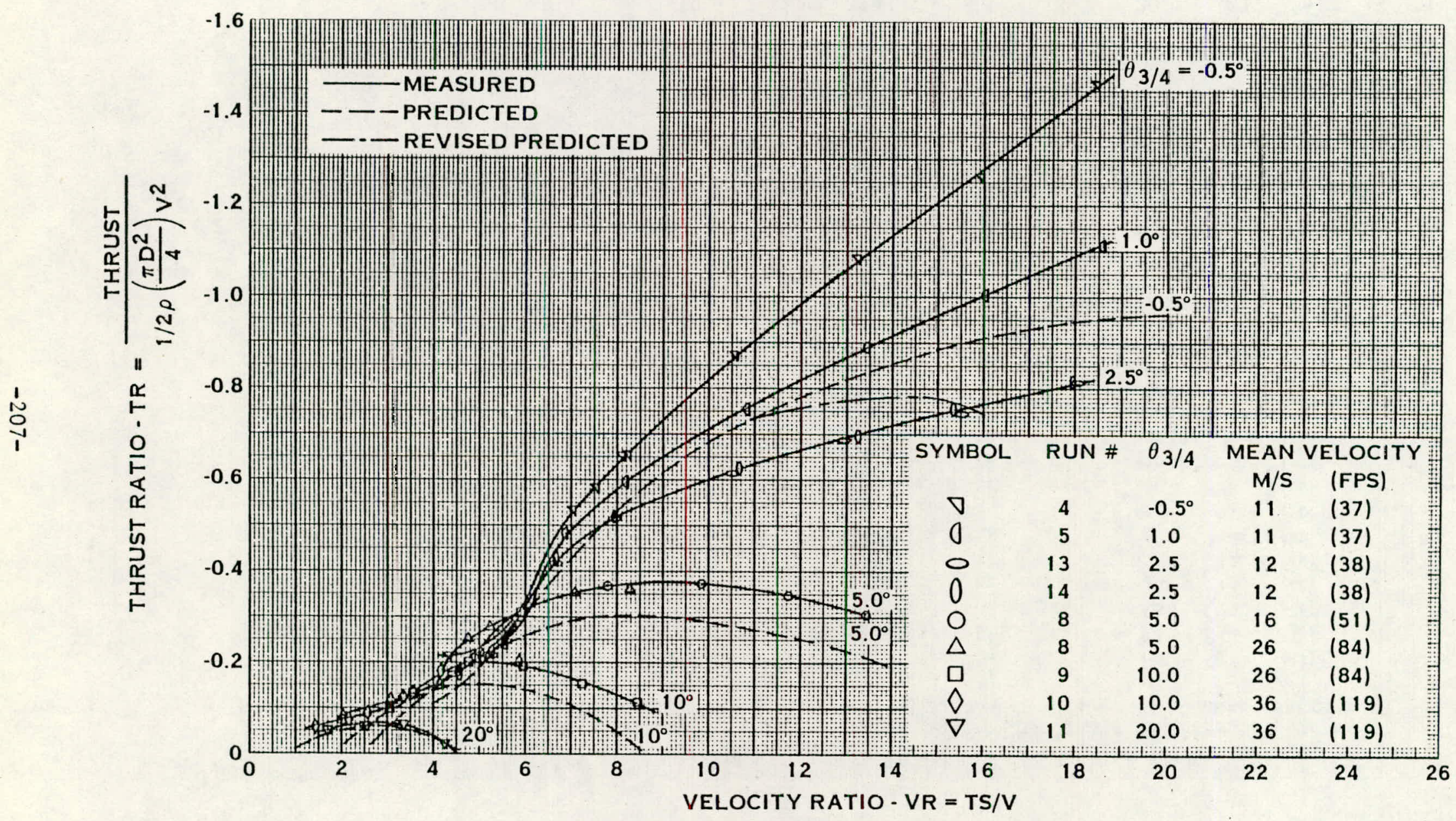

FIGURE 11-2B.COMPARISON BETWEEN MEASURED AND PREDICTED THRUST RATIO 
MODEL NO. 3

30 ACTIVITY FACTOR

NACA 230XX AIRFOIL SECTIONS 3 BLADES

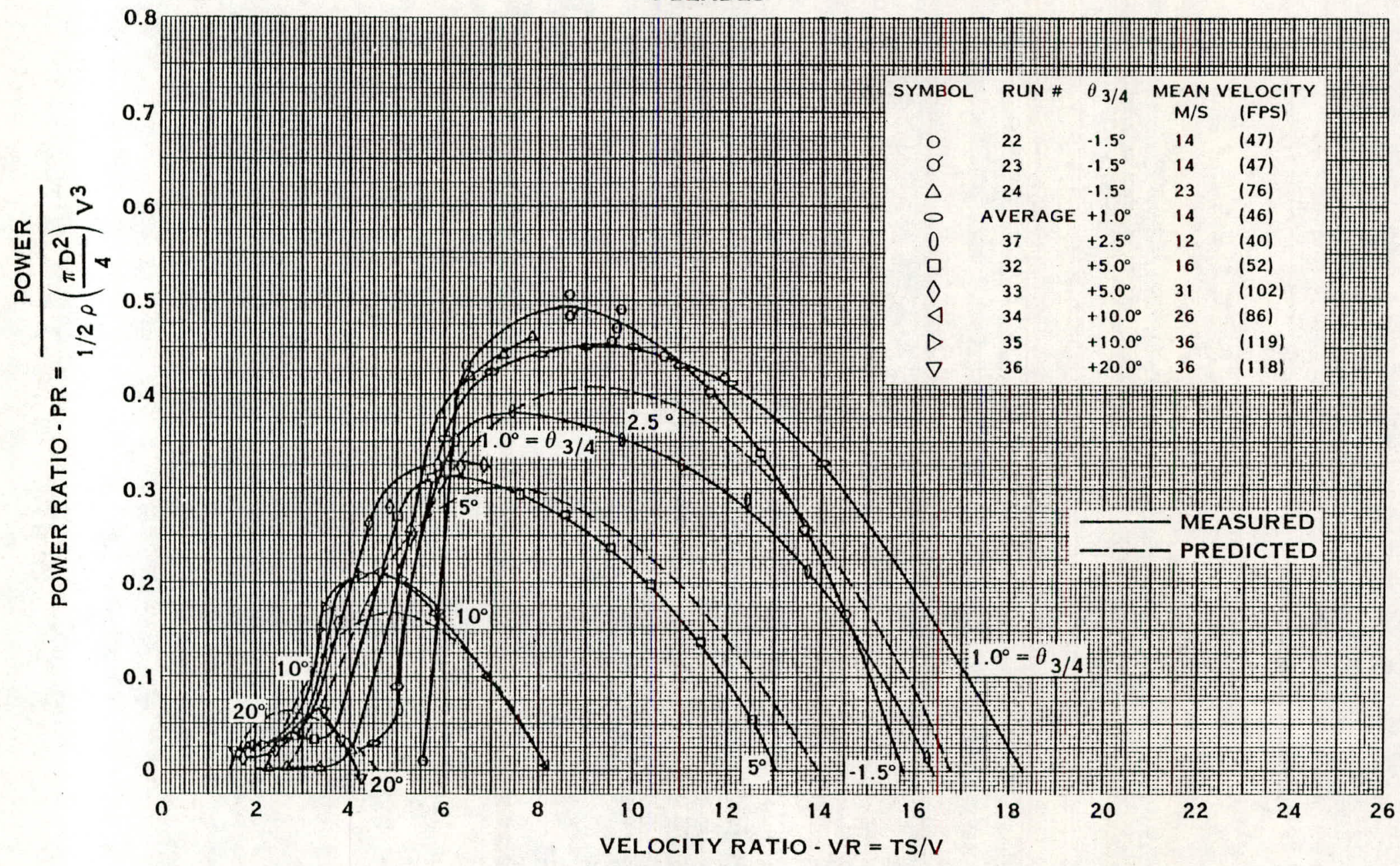

FIGURE 11-3A. COMPARISON BETWEEN MEASURED AND PREDICTED POWER RATIO 


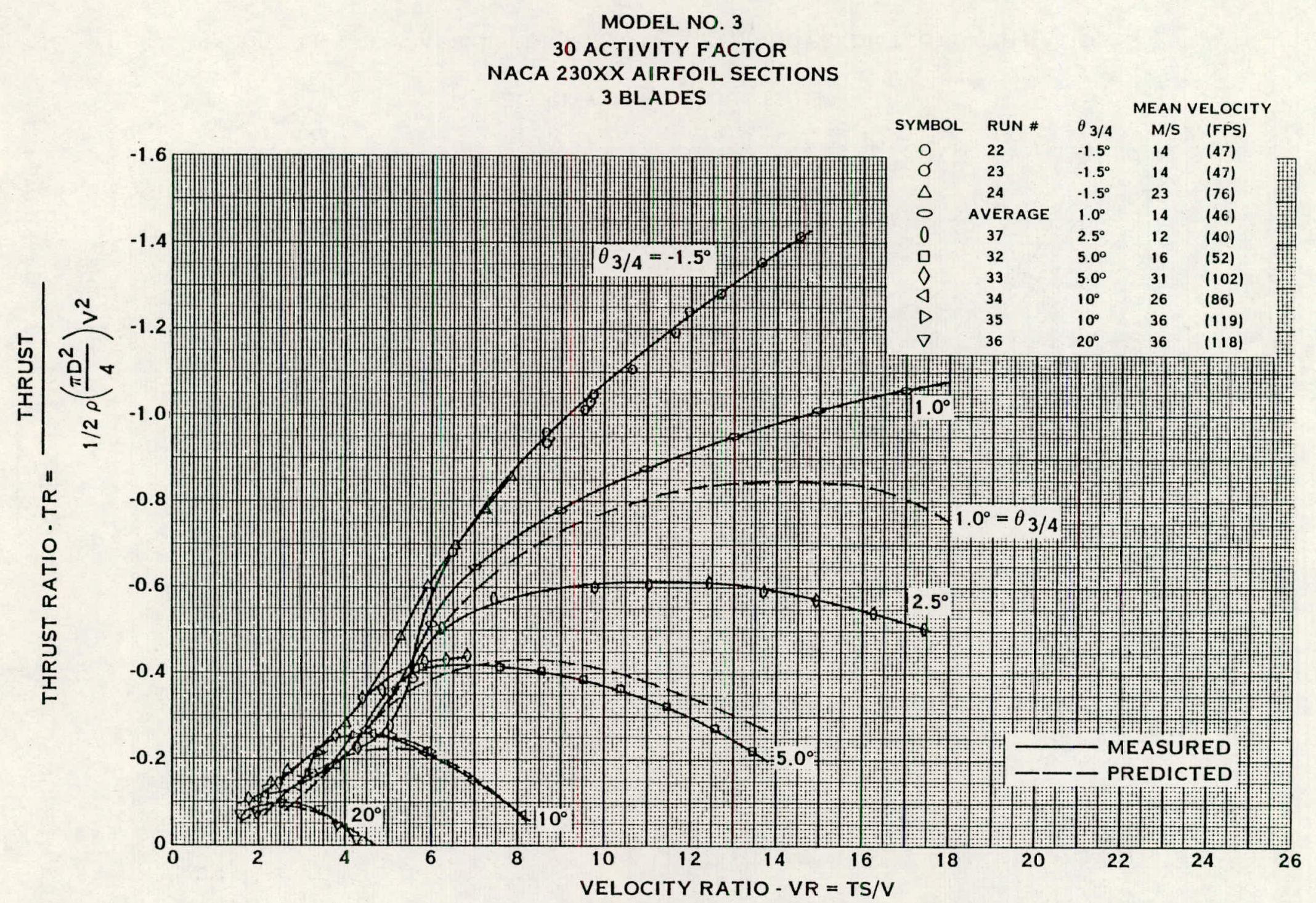

FIGURE 11-3BंCOMPARISON BETWEEN MEASURED AND PREDICTED POWER RATIO 


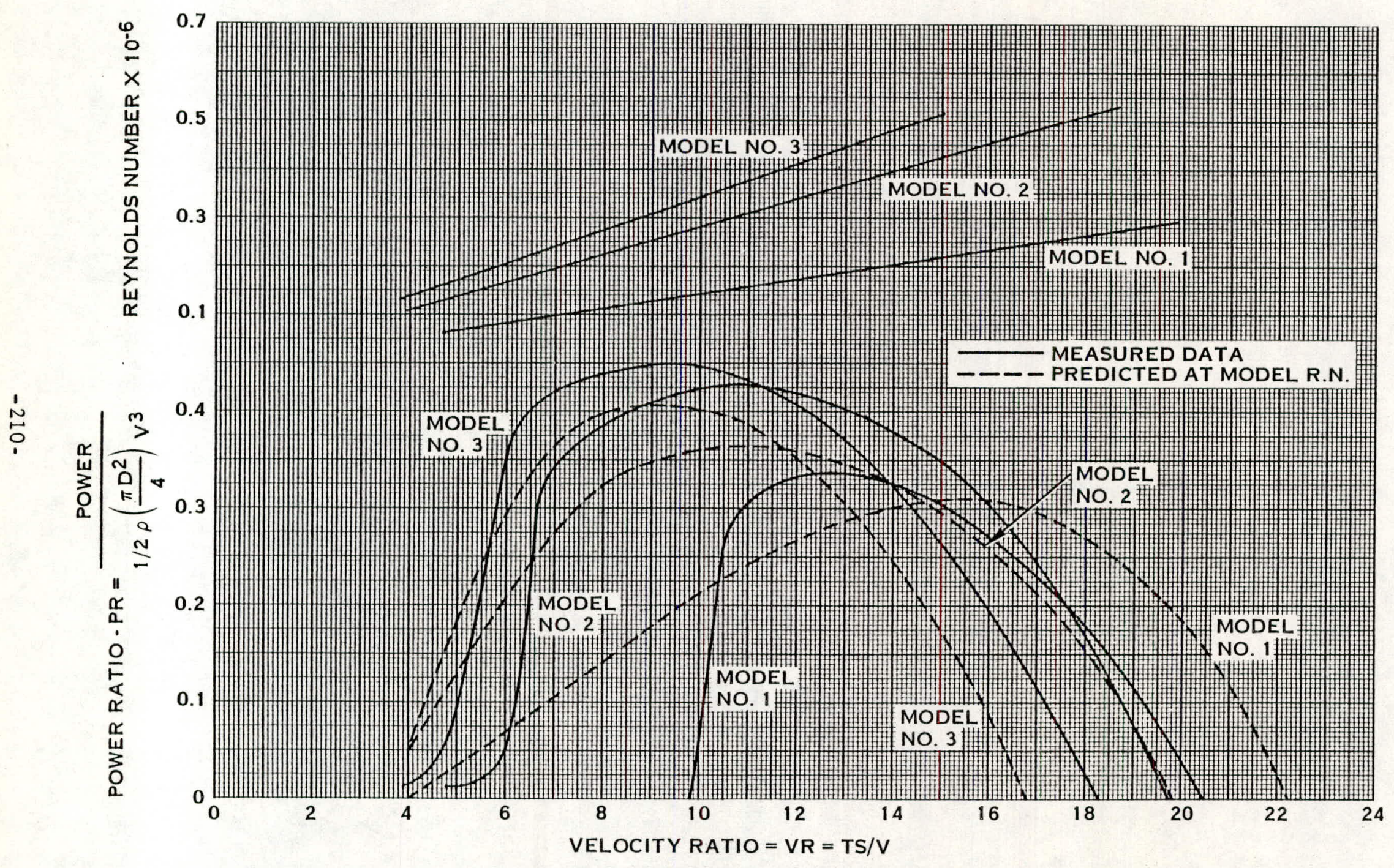

FIGURE 11-4.COMPARISON BETWEEN MEASURED AND PREDICTED POWER RATIO 


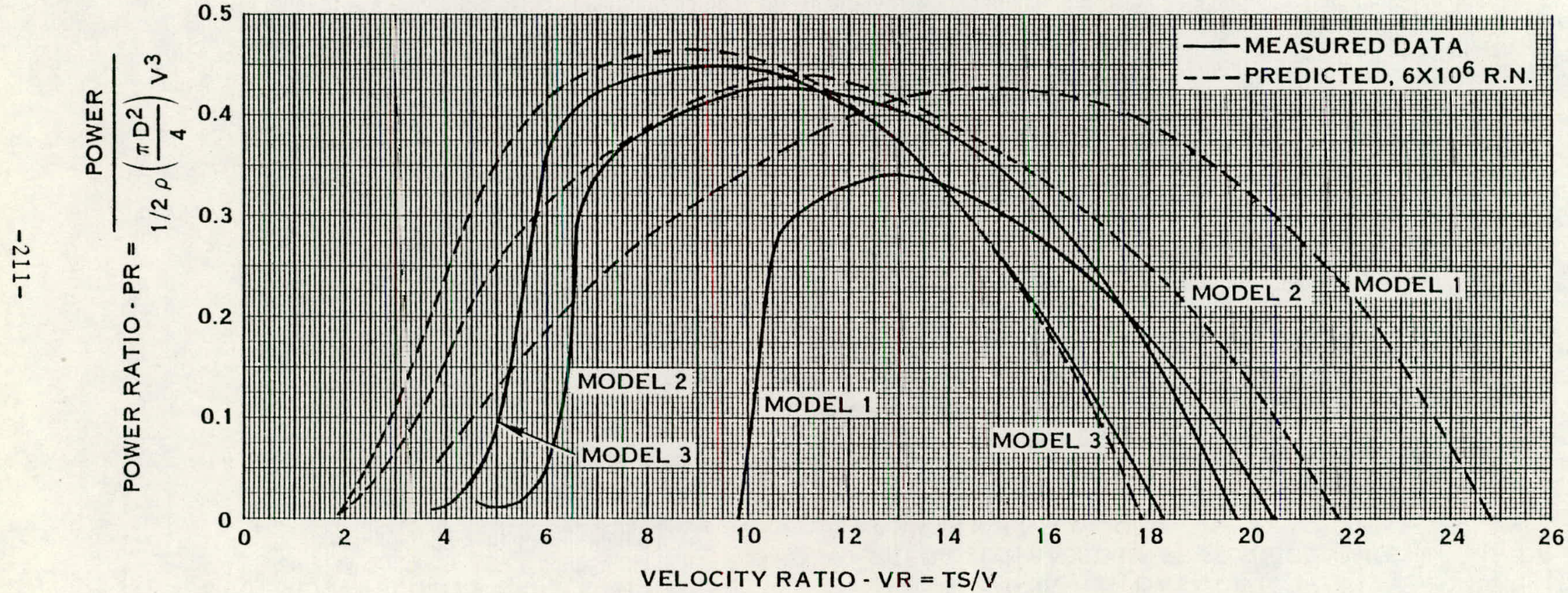

FIGURE 11-5. COMPARISON BETWEEN MEASURED MODEL AND PREDICTED FULL SCALE POWER RATIO 


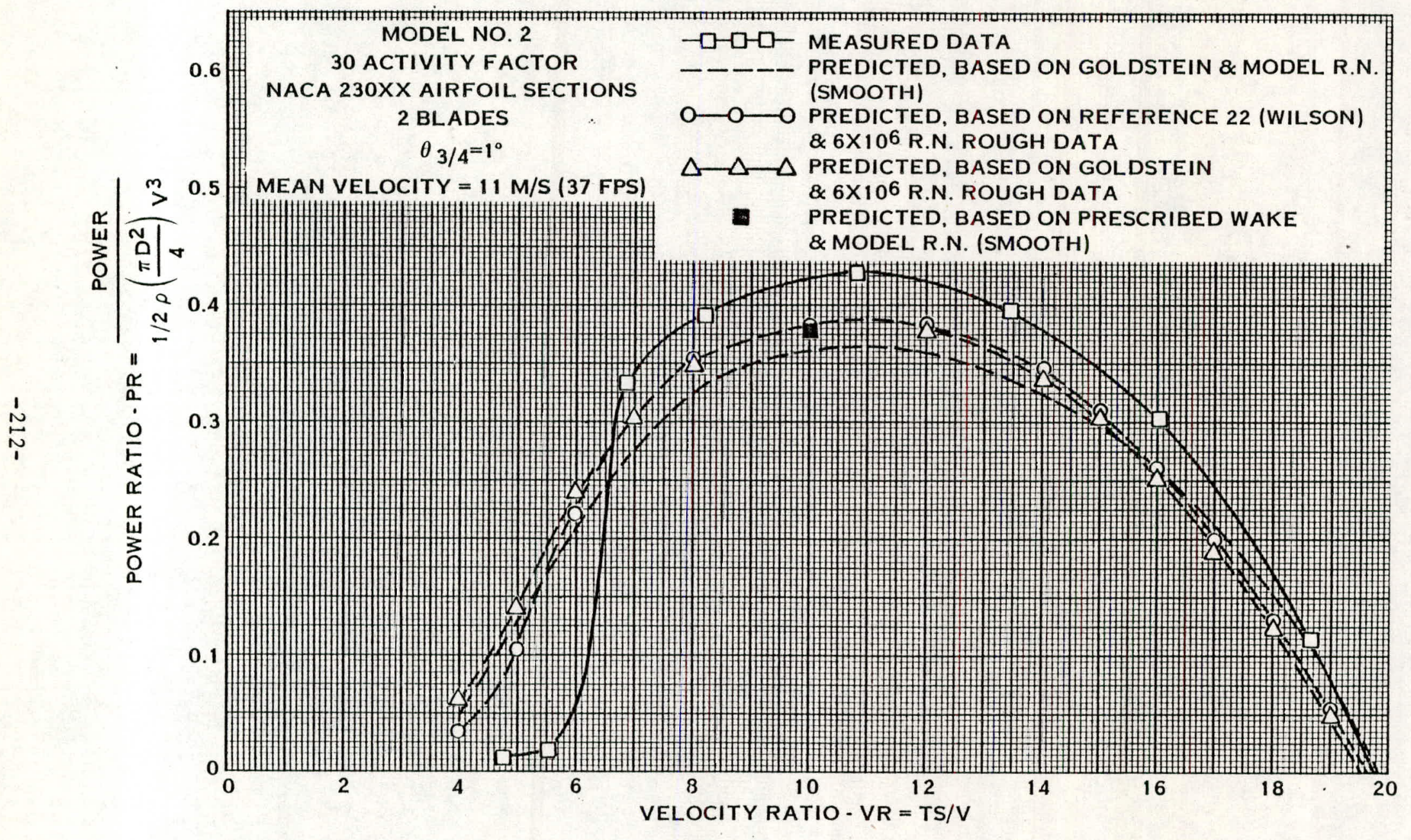

FIGURE 11-6. COMPARISON OF THE EFFECT OF DIFFERENT METHODOLOGIES ON PERFORMANCE 


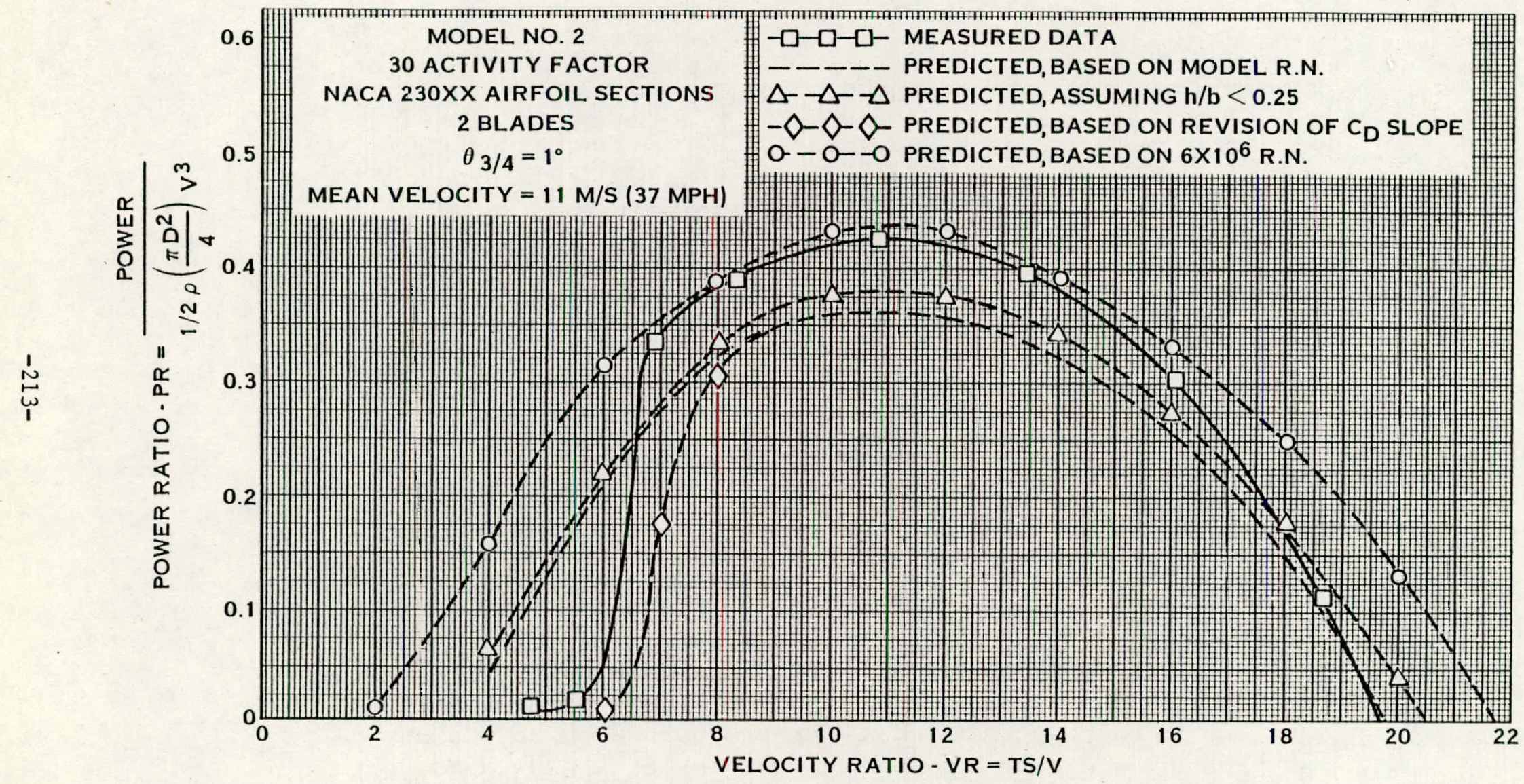

FIGURE 11-7. EFFECT OF AIRFOIL DATA ON PERFORMANCE 


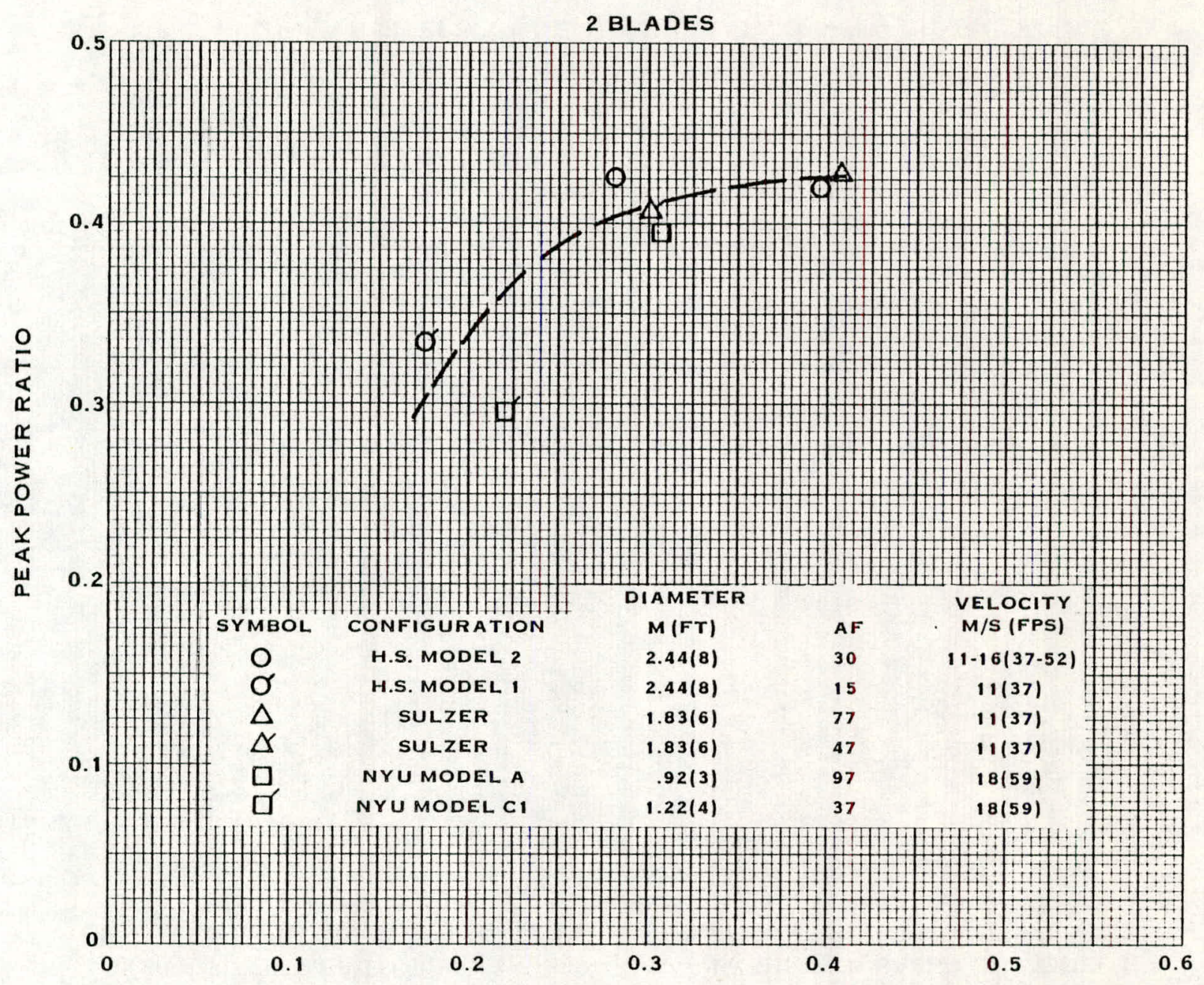

REYNOLDS NUMBER AT $3 / 4$ RADIUS X 10-6

FIGURE 11-8. MEASURED VARIATION OF POWER OUTPUT WITH REYNOLDS NUMBER 


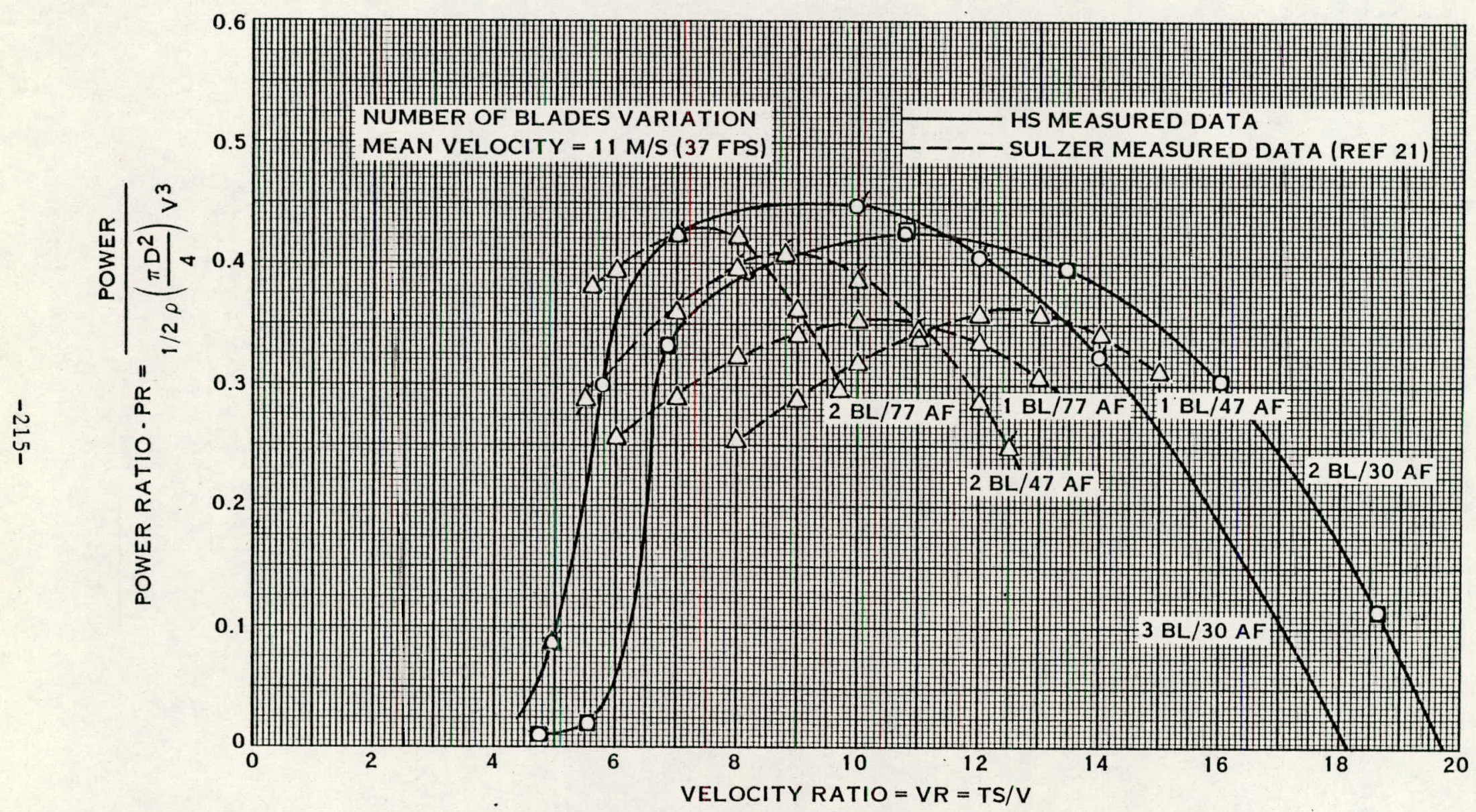

FIGURE 11-9. EFFECT OF NUMBER OF BLADES ON MEASURED POWER OUTPUT 


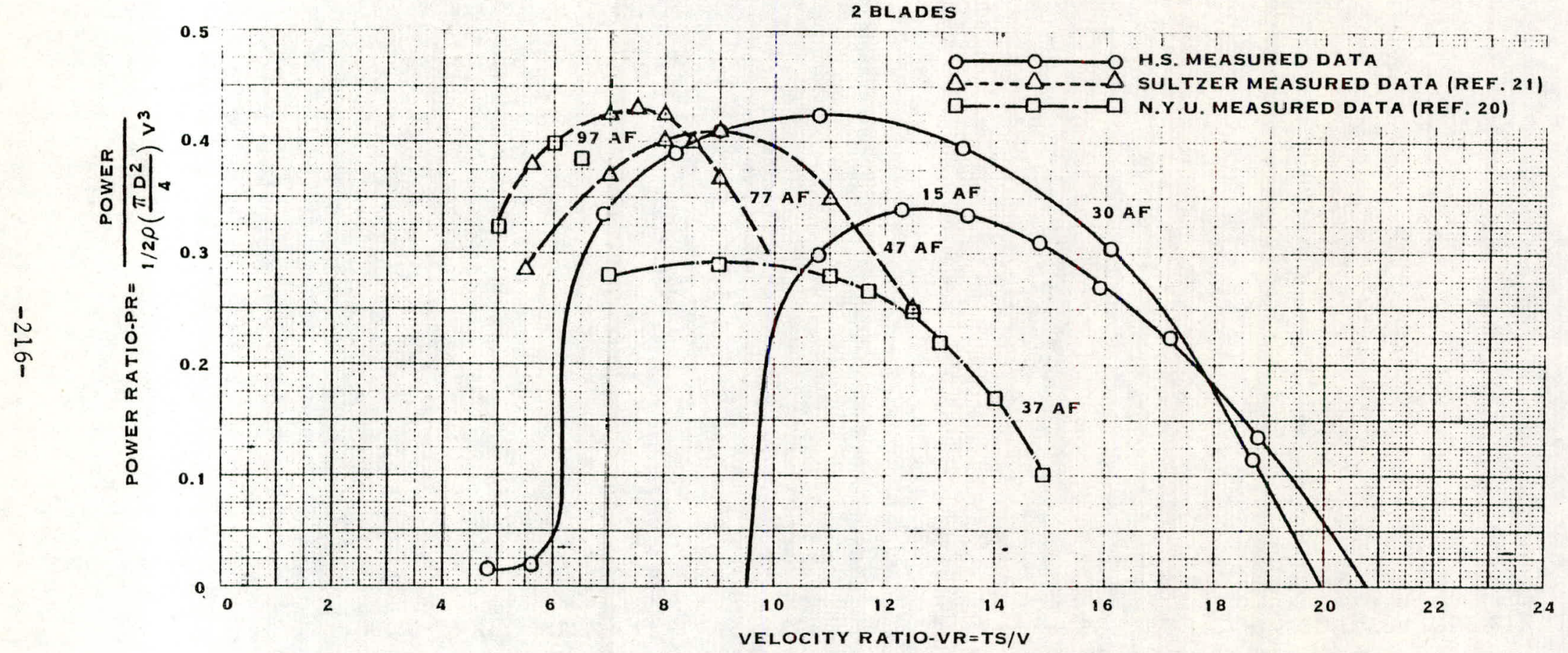

FIGURE 11-10. EFFECT OF ACTIVITY FACTOR ON MEASURED POWER OUTPUT 


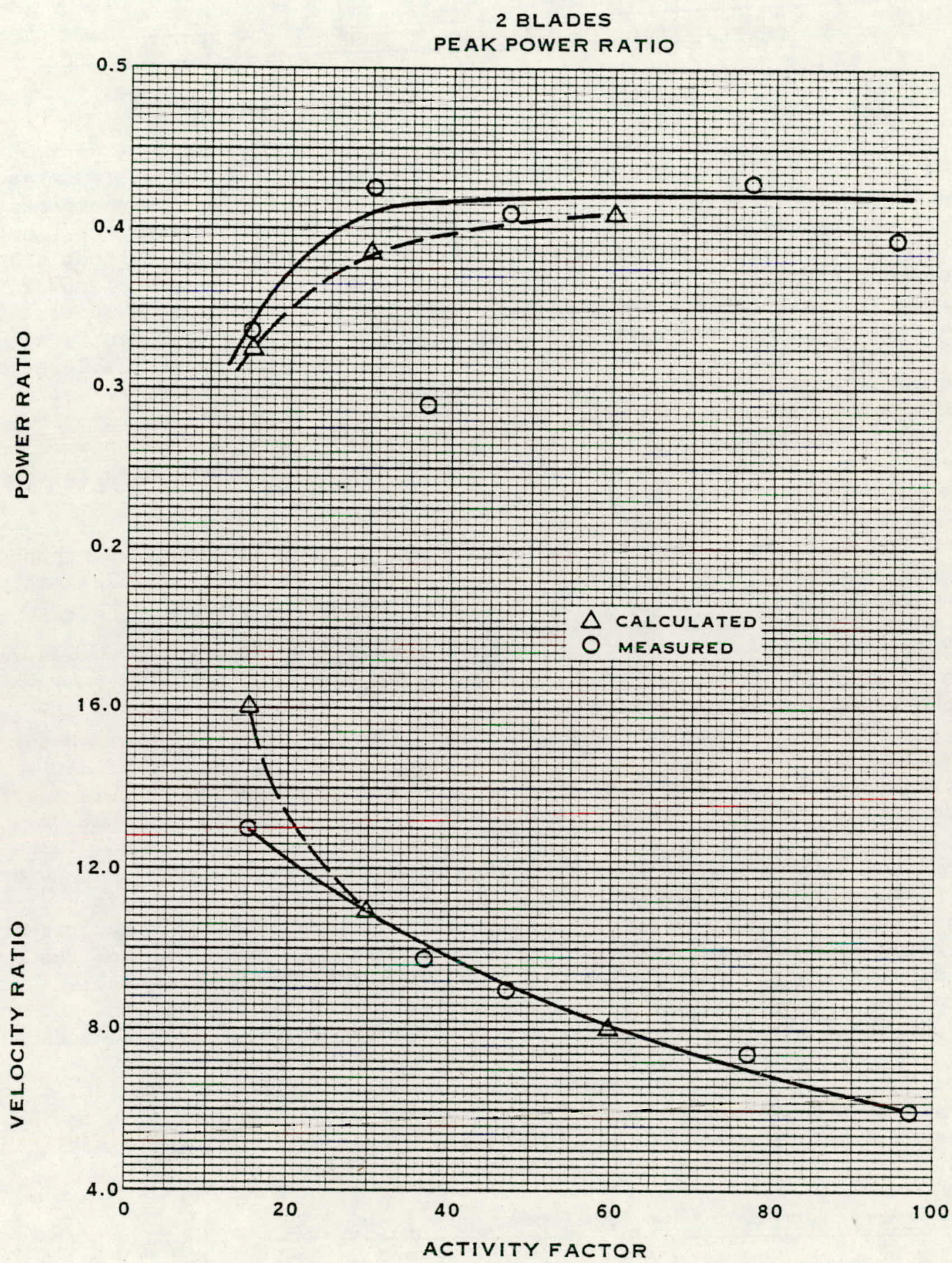

FIGURE 11-11. COMPARISON OF MEASURED AND CALCULATED EFFECT OF ACTIVITY FACTOR 
SECTION 12

\section{IDENTIFICATION OF AERODYNAMIC TECHNOLOGY NEEDS}

\subsection{INTRODUCTION}

The design of practical, cost effective wind energy conversion systems requires reliable technology in several disciplines related to the various components of the system including the aerodynamics of the wind turbine. Since the kinetic energy per unit volume of air at prevailing wind velocities throughout the world is relatively small, it follows that to produce practical levels of power, wind turbines are relatively large machines. For this reason, it is essential that the aerodynamic efficiency be as high as possible in order to minimize wind turbine dimensions and cost. Thus, there is a need to establish reliable aerodynamic technology for wind turbines. Towards this end, the Energy Research and Development Administration, ERDA, has undertaken a broad program to provide the required technology for the design of cost effective wind energy conversion systems ranging from the large sizes required for utility companies to the small sizes suited for private usage.

The experimental and analytical research program reported herein was sponsored by ERDA as one of the first steps toward the development of reliable aerodynamic technology.

The objectives of this program were to investigate, (1) the prime geometric variables of wind turbines operating over the practical range of velocity ratios, (2) the effect of inflow on wind turbine forces and moments, (3) the effect of Reynolds number of wind turbine performance, (4) the correlation of test measurements with calculated performance, (5) the potential application of new rotor aerodynamic methodology and (6) to establish from the results of this initial program, other areas of needed research.

While significant progress towards these objectives was achieved in this program as reported herein, the unfortunate early termination of the experimental phase caused by the model blade separation, prohibited much of the planned research from being accomplished. Moreover, the work which was completed and the problems encountered as well as the aerodynamic analyses performed in connection with other programs including the full scale design of the MOD-O and MOD-1 wind turbines have indicated several new areas of needed aerodynamic research and technology.

Accordingly, a recommended, follow-on research and technology program, aimed at accomplishing the original objectives presented above and including the new areas of required aerodynamic technology identified by this contractor since this program was initiated in 1975, is outlined below.

\subsection{CONTINUATION OF MODEL WIND TURBINE TEST PROGRAM}

The wind tunnel test reported herein has shown that model testing of wind turbines is feasible provided that large models are used to obtain Reynolds numbers of at least $3.5 \times 10^{5}$ and that testing is carefully controlled, particularly the tunnel velocity setting and the measuring systems are accurately calibrated to assure reliable data with a minimum of scatter. 
Accordingly, in view of the important testing to be accomplished to achieve the objectives listed above, it is recommended that the test program as originally planned be completed and that additional testing be included as outlined below with particular attention and importance being given to the added testing relating to simulation of full scale Reynolds number testing.

\subsubsection{Performance Test}

The performance testing should be continued to complete the program described in this report. The models include the 1 blade/30AF/230XX airfoils, the 2 blade/60AF/230XX airfoils and the 2 blade/60AF/44XX airfoils design. Thus with performance daca on these models and the performance data acquired on the three models reported herein, the solidity, number of blades and airfoil investigations as originally planned would be accomplished. In addition to these data the start-up characteristics of each model should be measured.

\subsubsection{Inflow Tests}

The effect of angular wind inflow on performance and on the resulting force and moment components is important in the design of the blades and the tower. Accordingly, both the 2 and 3 blade/30AF/230XX airfoil models should be tested with the plane of the turbine yawed over a range of angular from 15 to 60 degrees at the design blade angle and coverfing the velocity ratio range from start-up to shut-dows.

\subsubsection{Simulation of Full Scale Reynolds Number}

A Reynolds number simulation test is recommended on the 2 blade/15 AF, $30 \mathrm{AF}$ and $60 \mathrm{AF} / 230 \mathrm{XX}$ models by applying various coverages of grit on the leading edges in an attempt to simulate full scale Reynolds number on these models. If successful, this technique would permit wind tunnel investigations of blade geometry on relatively small, cost effective models to establish improved rotor performance for application to full scale wind turbine designs.

This same approach should be utilized to investigate the effect on performance of blade roughness due to manufacturing technique and service erosion.

\subsubsection{Flow Visualization Investigation}

The flow visualization test to define the wake characteristics of the 2 blade/ $30 \mathrm{AF} / 230 \mathrm{x}$ model should include a series of stroboscopic photographs of the wake covering both design and off-design operation, utilizing a multiple smoke jet rake to identify the tip vortex and inboard vortex sheet. This wake shapc data would be utilized to assess the prescribed wake and free wake rotor programs and to determine if the expanding. wake shape should be incorporated in the existing methodology which currently incorporates a non-expanding wake.

\subsection{WIND TURBINE PERFORMANCE COMPUTER PROGRAM}

The need exists for a reliable, complete aerodynamic design and performance prediction method which may be utilized by an emerging wind energy industry as a standard for selecting horizontal axis wind turbines. Accordingly, it is 
recommended that a computer deck be derived based on existing theory and including airfoil data packages containing generalized performance of the NACA 230XX and NACA $44 X X$ airfoil families covering a range of Reynolds number pertinent to wind turbine application. The program would be structured to permit the incorporation of wind shear, tower shadow and yawed inflow to more accurately represent the true environment. Moreover, the program would permit inputting any time-dependent, radial and circumferential, variation of the wind velocity. Thus the effect of wind variability on performance could be studied on a quasi-steady state basis.

Such a general aerodynamic performance program is not currently available and would add significantly to wind turbine design technology.

\subsection{WIND TURB INE AIRFOIL DATA}

The selection of optimum airfoil type for wind turbine blades is difficult to make in view of the limited data on thick airfoils. Very little data exists for thickness ratios above $25 \%$, although the structural design requirements lead to airfoil thickness ratios as high as $40 \%$ over the inner half of the blade. Moreover, the available data usually does not extend below 1 X $10^{6}$ Reynolds number. Thus experiments on model wind turbine cannot be adequately correlated with test data. Furthermore, because of these limited data, it is currently not possible to make direct comparisons of the various candidate airfoil types. Accordingly, two-dimensional wind tunnel tests should be conducted on airfoils such as the NACA 230XX, NACA 44XX and the new GA (W) general aviation airfoil families over an angle of attack range from -4 to $+20^{\circ}$, a Mach number range from 0.2 to 0.7 and a Reynolds number range from $.25 \times 10^{6}$ to $6 \times 10^{6}$. Each airfoil family should include a thickness ratio variation from $8 \%$ to $40 \%$. The effect of surface roughness should be established. The matrix of airfoils should be selected such that existing data need not be duplicated. These data would then be presented in generalized data packages for incorporation in wind turbine aerodynamic methodologies.

\subsection{ENVIRONMENTAL STUDIES}

Recent aerodynamic design analyses of large wind turbine rotors have indicated a significant effect of wind shear and tower shadow on the aerodynamic peformance. The effect has been to increase the diameters required to achieve a specified rated power output. Since the rotor diameter impacts heavily on wind turbine costs, it is essential that these environmental factors be investigated to accurately establish their effects on performance. To this end, it is recommended that the 2 and $3 \mathrm{bladed} / 30 \mathrm{AF} / 230 \mathrm{XX}$ models be tested in the wind tunnel in the presence of a simulated tower shadow and wind shear. The test data including both performance and stress measurements would be compared to the corresponding calculated data to assess the validity of the methodologies. 
The analytical and experimental research on wind turbines accomplished in this program provides an initial insight into the correlation of existing aerodynamic methodologies with experimental performance data and into the problems associated with wind tunnel tests on model wind turbines.

The reliability of existing theories has been briefly investigated. The calculated performance characteristics of wind turbines and the effect of the prime geometric and aerodynamic parameters have been generally confirmed by the test results. Finally, the need for developing very carefully controlled testing techniques on model wind turbines was clearly demonstrated.

These general conclusions are presented more specifically anc succinctly in the following statements covering the salient results of this program.

1. The parametric study results showed that a 2 bladed/30 AF/Trapezoidal Planform/NACA 230XX airfoils is close to an optimum configuration from both aerodynamic and structural considerations.

2. The prime blade shape parameters most affecting wind turbine performance and requiring experimental confirmation include number of blades, activity factor (solidity) and airfoil type. The prime aerodynamic parameters are Reynolds number and blade surface roughness and waviness.

3. The predicted performance of the six, $2.44 \mathrm{~m}(8-f t)$ diameter wind turbine models showed a strong effect of Reynolds number, particularly for the lowest activity factor model and for all models at low velocity ratios.

4. Reductions in calculated power output of the model wind turbines increased significantly with increases in inflow angles. Some of the trends with velocity ratio of the six force and moment components were somewhat irregular which may be associated with the airfoil performance at low Reynolds number.

5. The effect of low Reynolds number on the measured wind turbine model performance shnws an abrupt drop-uff in performance at low velocity ratios where the blade sections approach the maximum lift coefficient.

6. The experimental data indicate that essentially full scale peak power ratio levels are achieved at Reynolds numbers down to about $.35 \times 10^{6}$ compared to the predicted value of approximately $1 \times 10^{6}$.

7. The measured performance characteristics of the model wind turbines are generally as predicted. However, the reduction in power ratio level with decreasing Reynolds numbers above $3.5 \times 10^{5}$ is not as severe as predicted while the abrupt drop-off in power ratio at low velocity ratios is not predicted. 
8. Within the limits of the test data, the measured effects of activity factor and number of blades on performance is essentially as predicted, i.e. a 6 percent reduction in peak power ratio in going from 3 to 2 blades due to induced effects and a reduction in peak power ratio as activity factor falls below 30 due to Reynolds number effects. Analysis indicates no reduction in performance with activity factor below 60 at full scale Reynolds numbers.

9. The wind tunnel test demonstrated that model wind turbine testing provides an accurate measure of full scale wind turbine performance provided that Reynolds number of at least $3.5 \times 10^{-5}$ are achieved and that test wind velocities can be held within \pm .5 percent.

10. A significant portion of the discrepancy between calculation and test may be attributed to the inadequacy of the airfoil data generalization in the low Reynolds number range associated with this test program.

11. A single point evaluation of the Prescribed Wake Program incorporating a calculated, expanding, free wake for the energy extraction process improved the correlation with the test data indicating that the modified propeller theory may need further refinement.

12. The published method developed by Oregon State University yielded essentially the same performance levels as the modified Hamilton Standard method when the same airfoil data were utilized for both methods.

13. The potential for simulating full scale Reynolds numbers in tests on model wind turbines was indicated by use of a blade leading edge roughness strip to trip the boundary layer.

14. Further research should be conducted on an extensive family of model wind turbines in the wind tunnel with improved testing techniques to more completely evaluate the effect of geometric and aerodynamic parameters on performance and to fully explore the design velocity ratio range pertinent to full scale wind turbines. The program on flow visualization to define the wind turbine wake characteristics should be expanded to permit the derivation of wake geometry parameters for the Prescribed Wake Program. 
SECTION 14

REFERENCES

1. Goldstein, S., "On the Vortex Theory of Screw Propellers," Proceedings of the Royal Aeronautical Society, Series A, Vo1. 123, 1929.

2. Locke, C. N. H., "Application of Goldstein's Airscrew Theory to Design," British ARC, R\&M No. 1377, November 1930.

3. Hutter, Ulrich, "The Aerodynamic Layout of Wing Blades of Wind Turbines With High Tip Speed Ratio." Proceedings of the U.N. Conference on New Energy Sources, Vol. 7, Rome, 1961.

4. Iwasaki, M., "The Experimental and Theoretical Investigation of Windmills," Reports of Research Institute for Applied Mechanics, Kyusker University, Vol. II, No. 8, December 1953.

5. Anon, "Program for Conceptual Design, Parametric Analysis and Preliminary Design for Low Power (50-250. KW) and High Power (500-300 KW) Wind Generator Systems," Phase I Report of NASA Contract NAS3-194035, General Electric, Unpublished.

6. Rohrbach, C. and Worobel, R., "Performance Characteristics of Aerodynamically Optimum Turbines for Wind Energy Generators," Paper Preprint No. S-996, presented at the 31st Annual National Forum of the American Helicopter Society, Washington D.C., May 1975.

7. Landgrebe, A. J., "An Analytical Method for Predicting Rotor Wake Geometry," J.A.H.S., Vol. 14, No. 4, October 1969.

8. Jenney, D. S., Olson, J. R. and Landgrebe, A. J., "A Reassessment of Rotor Hovering Performance Prediction Methods," J.A.H.S., Vo1. 13, No. 2, April 1968.

9. Clark, D. R. and Leiper, A. C., "The Free Wake Analysis: A Method for the Prediction of Helicopter Rotor Hovering Performance," J.A.H.S., Vo1. 15, No. 1, January 1970.

10. Ladden, R. M., "Static Thrust Prediction Method Development," Technical Report AFFDL-TR-71-88, Vo1. II, Part 1, September 1971.

11. Glauert, H., "The Elements of Aerofoil and AlYscrew Theory," Cambridge University Press, 1937

12. Abbott, I. H., von Doenhoff, A. E. and Stevens, L. S., Jr., "Summary of Airfoil Data," NACA Report 824, March 1945.

13. Wortmann, F. X., "The Quest for High Lift," AIAA Paper No. 74-1018, Sep tember 11-13, 1974.

14. Liebeck, R. H., "A Class of Airfoils Designed for High Lift in Incompressible Flow," AIAA Paper No. 73-86, January 10-12, 1973. 
15. McGhee, R。 J. and Beasley, W。 D., "Low-Speed Aerodynamic Characteristics of 17-Percent - Thick Airfoil Sections Designed for General Aviation Applications," NASA TN D-7428, December 1973.

16. Jacobs, E. N. and Sherman, A., "Airfoil Characteristics As Affected By Variations of the Reynolds Number," NACA Report 586, 1937.

17. Braslow, A..L. and Knox, A. E.: Simplified Method for Determination of Critical Height of Distributed Roughness Particles for Boundary-Layer Transition as Mach Numbers from 0 to 5. NACA 4363, 1958.

18. Robinson, H. L., "High-Speed Investigation of Skin Wrinkles on Two NACA Airfoils," NACA Technical Note No. 1121, August 1946.

19. Pope, A., "Low Speed Wind Tunnel Testing," John Wiley and Sons, Inc., 1966 Edition.

20. N.Y.U., Final Report on the Wind Turbine. Office of Production, Research and Development, War Production Board; PB 25370, Washington, D.C., January 31, 1946.

21. Sulzer, P. Q., Unpublished Data on 1.8m (6 ft.) Wind Turbines.

22. Wilson, R. E. and Lissman, P.B.S., "Applied Aerodynamics of Wind Power Machines," Oregon State University, May 1974. 


\section{SECTION 15}

ACKNOWLEDGEMENT

The authors wish to extend their appreciation to Mr. P. Sulzer for granting permission to use his unpublished wind turbine test data. 
SECTION 16

SYMBOLS

$$
\begin{aligned}
& \text { AF - blade activity factor }=\frac{100,000}{16} \int_{\text {hub }}^{\text {tip }} \frac{b}{D}(r / R)^{3} d(r / R) . \\
& \text { b - blade section chord, m ( } f t \text { ) } \\
& C_{L}-\quad \text { lift coefficient } \\
& \text { D - wind turbine diameter, m ( } f t \text { ) } \\
& \text { h - blade section thickness, m ( } f t \text { ) } \\
& \text { L/D - ratio of } 1 \text { ift/drag } \\
& \text { MR - Horizontal Plane Moment/ }\left(\rho / 2 \cdot \frac{\pi D^{2}}{4} \cdot V^{2} \cdot \frac{D}{2}\right) \\
& \text { NR - Inplane Horizontal Force/ }\left(\rho / 2 \cdot \frac{\pi D^{2}}{4} \cdot V^{2}\right) \\
& P R-\quad \text { Power/ }\left(\rho / 2 \cdot \frac{\pi D^{2}}{4} \cdot V^{3}\right) \\
& \text { I - local blade section radius, m ( } f t) \\
& R \text { - blade tip radius, } m \text { ( } f t \text { ) } \\
& \text { R.N. - Reynolds number, } \rho \frac{V_{R}}{\mu} b \\
& S R \text { - Inplane Vertical Force/ }\left(\rho / 2 \cdot \frac{\pi D^{2}}{4} \cdot V^{2} \cdot \frac{D^{2}}{4}\right. \\
& \text { TR - Thrust/ }\left(\rho / 2 \cdot \pi D^{2} \cdot V^{2}\right) \\
& \text { TS - Wind Turbine Tip speed, } \mathrm{m} / \mathrm{s} \text { (fps). } \\
& \mathrm{V} \text { - Wind velocity, m/s (fps) } \\
& V_{R}-\quad \text { Blade sectional resultant velocity, } m / s \text { (fps) } \\
& \mathrm{VR}-\quad \text { Velocity ratio }=\mathrm{TS} / \mathrm{V} \\
& \text { YR - Vertical Plane Moment }\left(\rho / 2 \cdot \frac{\pi D^{2}}{4} \cdot V^{2} \cdot \frac{D}{2}\right) \\
& \mu \text { - Viscosity of air } N-\sec / \mathrm{m}^{2}\left(1 \mathrm{~b}-\mathrm{sec} / \mathrm{ft} \mathrm{t}^{2}\right) \\
& \rho-\quad \text { Density of air } N-\sec ^{2} / \mathrm{m}^{4}\left(1 \mathrm{~b}-\sec ^{2} / \mathrm{ft} t^{4}\right)
\end{aligned}
$$

Abbreviations :

GA(W)-1 General Aviation (Whitcomb) - number 1 


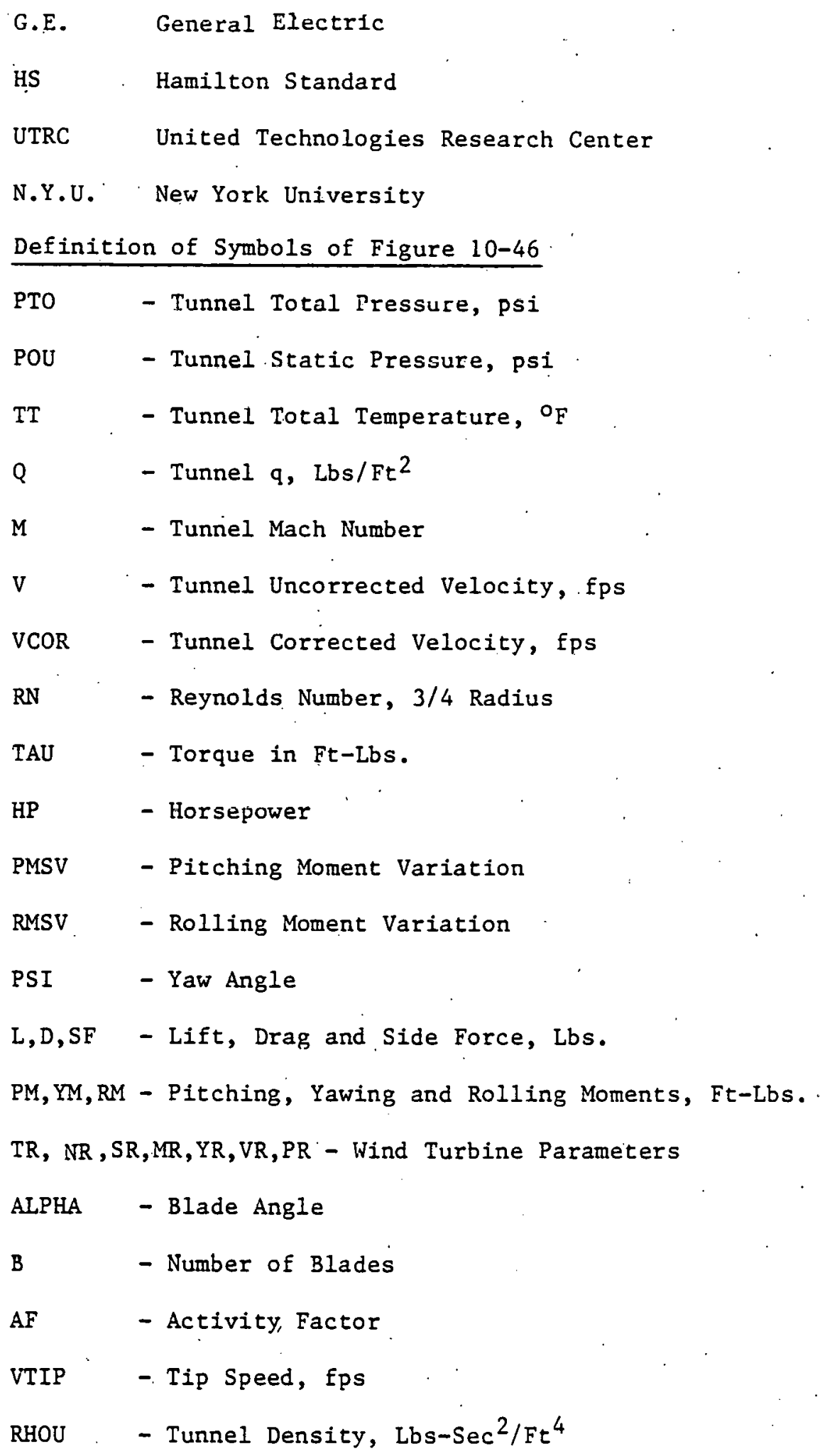


APPENDIX A

ORIGINAL TEST SCHEDULE

\section{Al GENERAL DESCRIPTION OF TEST PROGRAM}

In order to accomplish the objectives of this program, four separate two-bladed wind turbine models have been designed and fabricated. One of these models will also be tested in one and three-bladed configurations. Thus, a total of six separate wind turbine configurations will be examined. These are:

Activity Factor No. of Blades Airfoil Section

$\begin{array}{rrr}15 & 2 & 230 \mathrm{XX} \\ 30 & 2 & 230 \mathrm{XX} \\ 30 & 1 & 230 \mathrm{XX} \\ 30 & 3 & 230 \mathrm{xX} \\ 60 & 2 & 230 \mathrm{XX} \\ 60 & 2 & 44 \mathrm{XX}\end{array}$

Each of these models will be tested over a tip speed, blade angle and freestream velocity range. The ranges are:

$\begin{array}{lrl}\text { Velocity } & 35 \text { to } 5 \mathrm{~m} / \mathrm{s} & (116 \text { to } 16 \mathrm{fps}) \\ \text { Tip Speed } & 213 \text { to } 61 \mathrm{~m} / \mathrm{s} & \begin{array}{l}(700 \text { to } 200 \mathrm{fps}) \\ \text { Blade Angle }\end{array}\end{array}$

Specific increments of each of these operating variables have been selected so that the complete performance of each turbine can be defined within structural limits.

In addition to obtaining the basic performance of each turbine other specific areas of operation will also be investigated. With the 30 activity factor, 2 bladed wind turbine inflow angles of 15, 30, 45 and 60 degress will be tested while all the fozces acting on the turbine are measured.

With the 60 activity factor blades, the effect of roughness will be investigated. This will be done by sprinkling very fine carborundum dust near the leading edge of both 60 activity factor blades and noting the change in performance. Since different airfoils are used on these models, the effect of roughness on airfoil performance can be evaluated. As a final step, flow visualization photographs will be taken of the twobladed, 30 activity factor turbine while it is operating near its design operating condition. At this condition, a series of photographs will be taken to define the path of the tip vortex and the inboard vortex sheet. This will be done by the use of a multiple smoke jet rake located upstream of the wind turbine and stroboscopic lighting.

All of this testing will be conducted in the $5.5 \mathrm{~m}$ ( $18 \mathrm{ft}$.) throat of the United Technologies Research Laboratories (UTRC) subsonic wind tunnel. A special test rig (shown in figures $A-1$ and $A-2$ ) and support pylon will 
be provided. for mounting the test turbines. The rig will incorporate a turbine loading system, utilize the main tunnel six component balance for measuring moments and forces and is capable of being yawed. In this facility, the data can be automatically recorded and reduced online so that the performance of each wind turbine can be monitored as it is being obtained.

Briefly, the test program will consist of the following:

a) Basic Test

$30 \mathrm{AF} / 230 \mathrm{XX}$ airfoil turbine, tested as 1,2 and 3 blades

$15 \mathrm{AF} / 230 \mathrm{XX}$ airfoil turbine, tested as 2 blades

$60 \mathrm{AF} / 230 \mathrm{XX}$.airfoil turbine, tested as 2 blades

$60 \mathrm{AF} / 44 \mathrm{XX}$ airfoil turbine, tested as 2 blades

Thus, a total of six configurations will be investigated. Each configuration will be tested over a range of velocities and blade angles to completely define its performance. A total of 43 runs will be made in this test.

b) Reynolds Number Effect

$30 \mathrm{AF} / 230 \mathrm{XX}$ airfoil turbine, tested as 2 blades

The total number of runs specified during the Basic Test will allow the assessment of the basic effect of Reynolds number on turbine performance. A more detailed study will be made by testing the above configuration at three additional velocities for a total of 3 runs.

c) Inflow Variation

$30 \mathrm{AF} / 230 \mathrm{XX}$ airfoil turbine, tested as 2 blades

This configuration will be tested at four inflow angles, design blade angle, and two tunnel velocities. Thus, a total of 8 runs will be made to determine the effect of inflow.

d) Roughness Effect

$60 \mathrm{AF} / 230 \mathrm{XX}$ airfoil turbine, tested as 2 blades

$60 \mathrm{AF} / 44 \mathrm{XX}$ airfoil turbine, tested as 2 blades

\section{A2 TEST SCHEDURE}

Each of the models will be tested over the range of blade angles, RPM's and velocities shown in the tables below. These velocities and angles have been carefully selected to provide as much test coverage as possible while considering such parameters as Reynolds number and critical speeds. In each case, an overlapping test coverage, as shown in figure $\mathrm{A}-3$, has been provided. This will allcw the complete performance of each model to be established. 
TABLE I

Activity Factor $=15,2$ Blades

$\begin{array}{lrr}\text { Blade Angle } & \text { Velocity, m/s (fps) } & \text { RPM } \\ \text { Design Angle } & 7.13,10.4,14.3(23.4,34,47) & 1671 \\ & & 1433 \\ & & 1194 \\ & & 955 \\ & & 716 \\ 5^{\circ} & 14.3(47) & 400 \\ & & 1671 \\ & & 1433 \\ & & 119 / 1 \\ 10^{\circ} & & 955 \\ & & 716 \\ & & 400 \\ & & 955 \\ & & 716 \\ & & 400\end{array}$

Avoid continuous running between 955 and 716 RPM.

Activity Factor $=30,2$ Blades

Blade Angle

Velocity, m/s (fps)

$\underline{R P M}$

Design Angle 4.9, 7.5, 10.7, 15.2, 21.3 (16, 25, 35, 50, 70) 1671

1433

1194

955

716

477

$5^{\circ}$

$14.3(47)$

1671

1433

1194

955

716

400

$10^{\circ}$

$24.4,35.4(82,116)$

1671

1433

1194

955

716

477 
Blade Angle

Velocity, $\mathrm{m} / \mathrm{s}$ (fps)

$\underline{\mathrm{RPM}}$

$20^{\circ}$

35.4 (116)

1194

955

716

477

Continuous running between 955 and 716 RPM is to be avoided.

$\underline{\text { Activity Factor }=30,3 \text { Blades }}$

Blade Angle

Velocity, $\mathrm{m} / \mathrm{s}$.(fps)

$\underline{R P M}$

Design Angle

$13.4,21.9,30.5(44, .72,100)$

1671

1433

1194

955

716

477

$5^{\circ}$

$15.5,30.5(51,100)$

1671

1433

1194

955

716

$\gamma$

$477^{\circ}$

$10^{\circ}$

$25.9,35.4(85,116)$

1671

1433

1194

955

716

477

$20^{\circ}$

$35.4(116)$

1671

1433

1194

955

716

Continuous running between 955 and 716 RPM is to be avoided.

30 Activity Factor, 1 Blade

Blade Angle

Velocity, $\mathrm{m} / \mathrm{s}$ (fps)

$\underline{\text { RPM }}$

Design Angle

8.8 (29)

1671

1433

1194

955

716

400

Avoid continuous running between 955 and 716 RPM. 
60 Activity Factor, 2 Blades

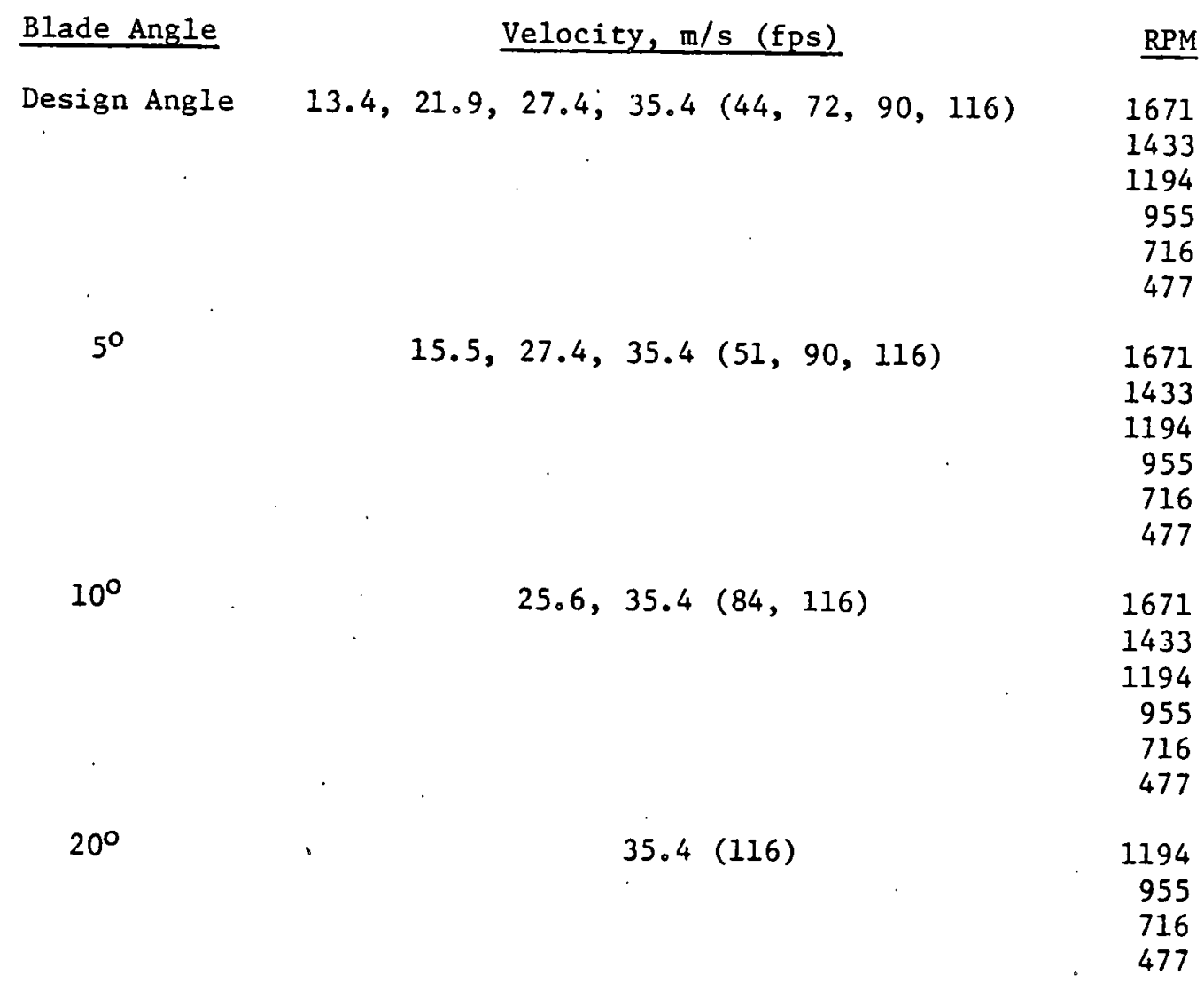

Continuous running below 477 RPM is to be avoided.

The above tables represent the run schedule for the performance portion of this test program. As test data are accumulated, it may be necessary to adjust the above schedules to provide the best test coverage.

In addition to the basic performance tests listed above, the basic 30 activity factor model of this program will be tested at inflow angles of 15, 30, 45 and 60 degrees over the same range of tip speeds as the performance tests and two forward velocities at the design blade angle. During this testing all the forces and moments acting on the wind turbine will be measured on the six-component balance. Here again, as in the case of the performance testing, the experimental data will be superimposed on the calculated results to compare trends.

In order to fully explore the effect of Reynolds number, the 30 activity factor, 2 bladed wind turbine will be tested over an extended range of velocities. It can be noted from the previous tables that this model will be tested at five velocities at the design angle. This will allow the test data to be extrapolated and interpolated over as wide a range as possible.

To investigate the effect of leading edge roughness and fixed transition on performance, the two 60 activity factor wind turbines will be tested 
with fixed leading edge transition. In order to do this, .0025 inch or larger carborundum grains will be applied to the first eight percent of the upper and lower surface of the blade leading edge. The grains will be spread to cover 5 to 10 percent of this area. It is felt that this roughness is considerably more severe than that caused by the usual manufacturing irregularities or deterioration in service.

In order to investigate this effect; the testing of the two 60 activity factor turbines will be repeated at the design angle and the previously run tip speeds and velocities. In this manner, the effect of roughness on overall performance can be evaluated. Since these two turbines incorporate different airfoil sections, the effect of roughness in airfoil section will also be evaluated.

\section{A2.1 Flow Visualization}

As the last phase of the test program, flow visualization data will be obtained for assessing the Prescribed Wake Program and Rotor Wake Geometry Program. For this testing a multiple jet smoke rake will be used to define the tip vortex and inboard sheet path of the basic 30 activity factor wind turbine model. Based on the results of the performance testing, several selected operating conditions near the peak power ratio point will be investigated.

The technnique to be used will be to deploy a multiple jet smoke rake near the wind turbine. The rake consists of multiple probes for mixing jets of anhidrous ammonia and sulphur dioxide to produce a thin smoke filament. The rake will be positioned to define the path of the tip and inboard sheet vortices-simultaneously. By stroboscopic photography the path of each vortex may be accurately defined by actual measurements of the vortex displacements on the photographs. A typical photograph of a propeller tip vortex is shown in figure A-4. Photographs are usually taken at various azimuthal positions of one blade for one revolution of the propeller. In this program, photographs will be taken at every 30 degrees of azimuth.

Roughness will be applied to each configuration in the form of carborundum dust. Each configuration will be tested at one blade angle and two velocities for a total of 4 runs.

e) Flow Visualization

$30 \mathrm{AF} / 230 \mathrm{XX}$ airfoil turbine, tested as 2 blades

Flow visualization will be used to establish the tip and inboard vortex filaments. 


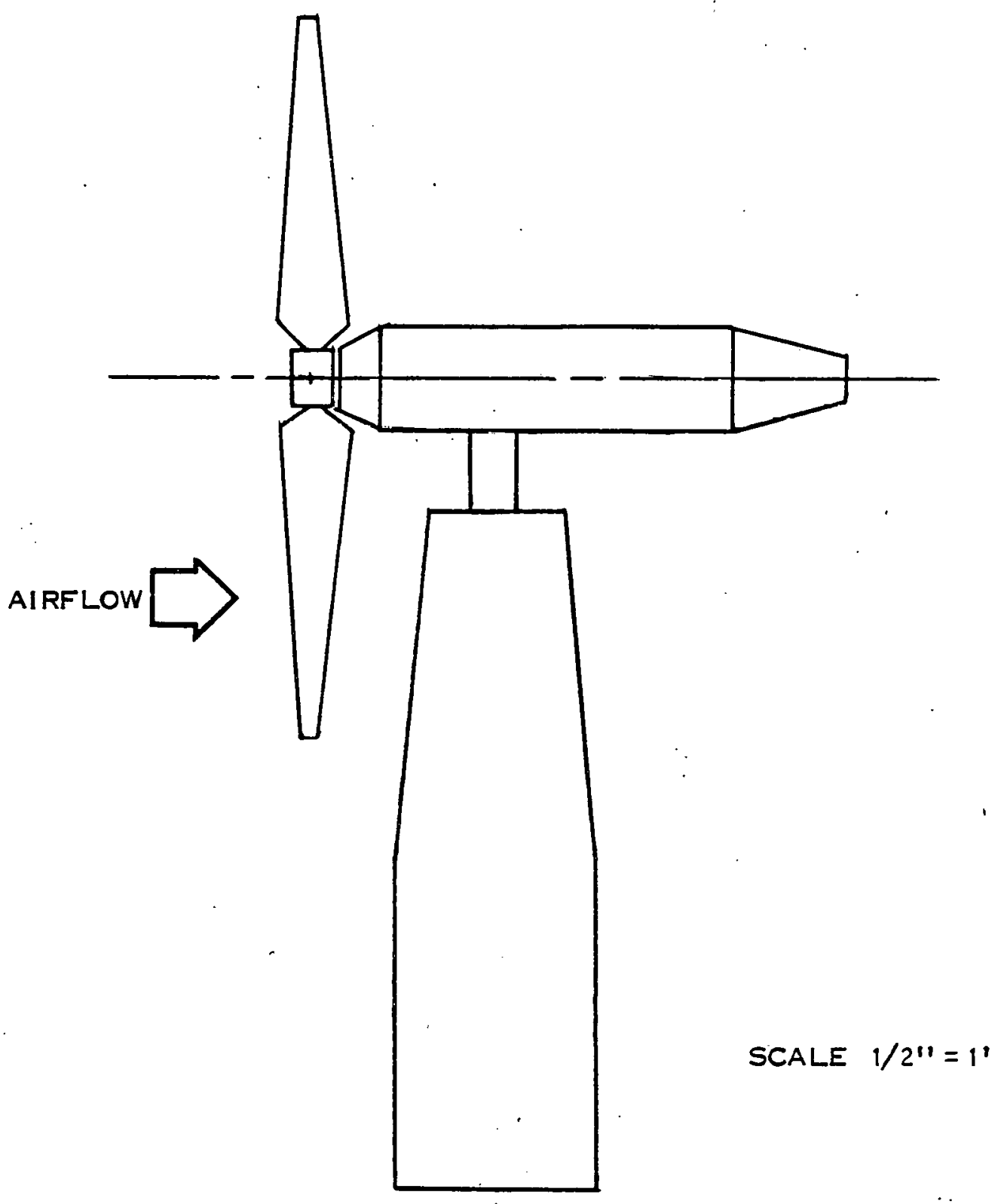

FIGURE A-1 SIDE VIEW OF TEST RIG 


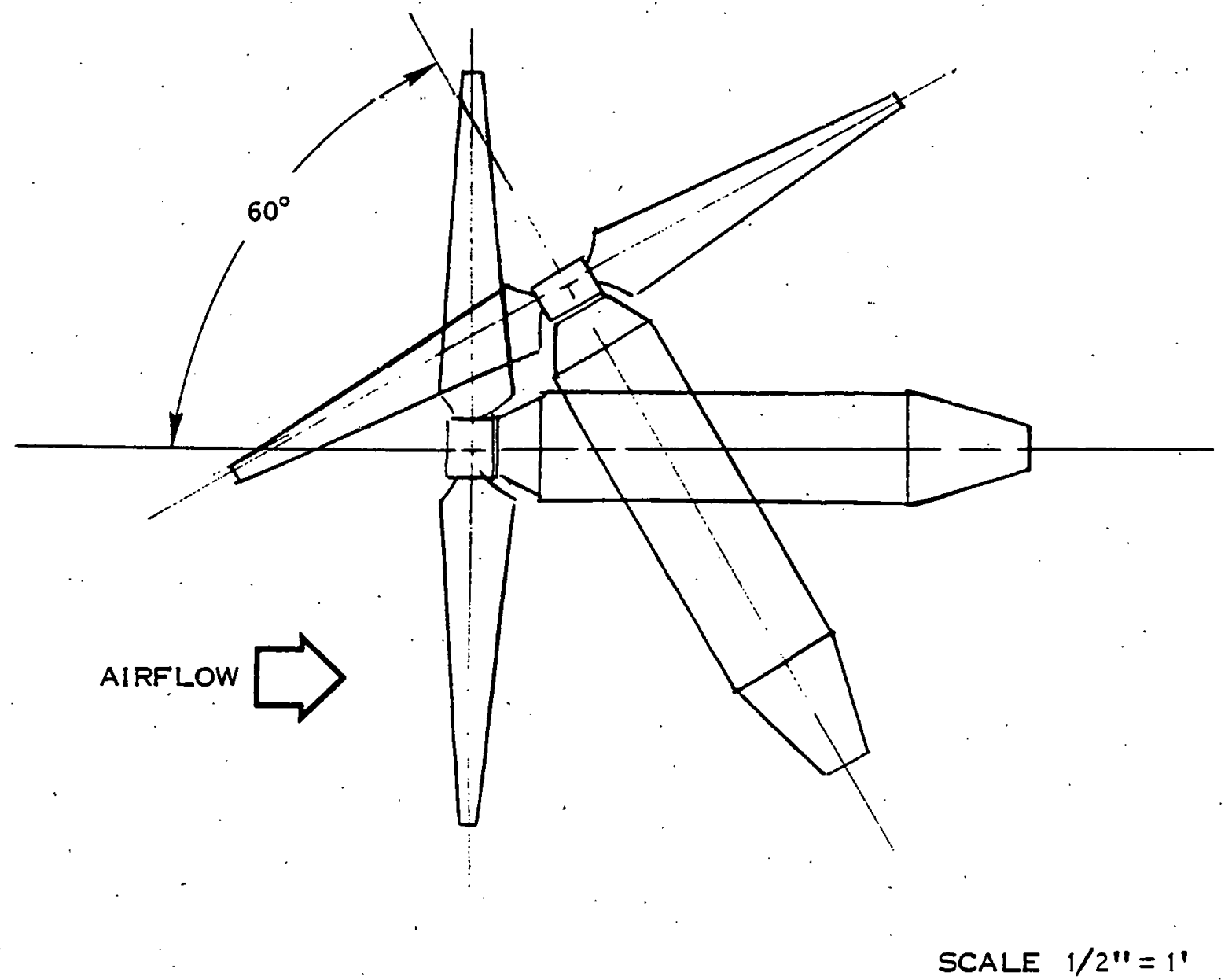

FIGURE A-2 TOP VIEW OF TEST RIG 

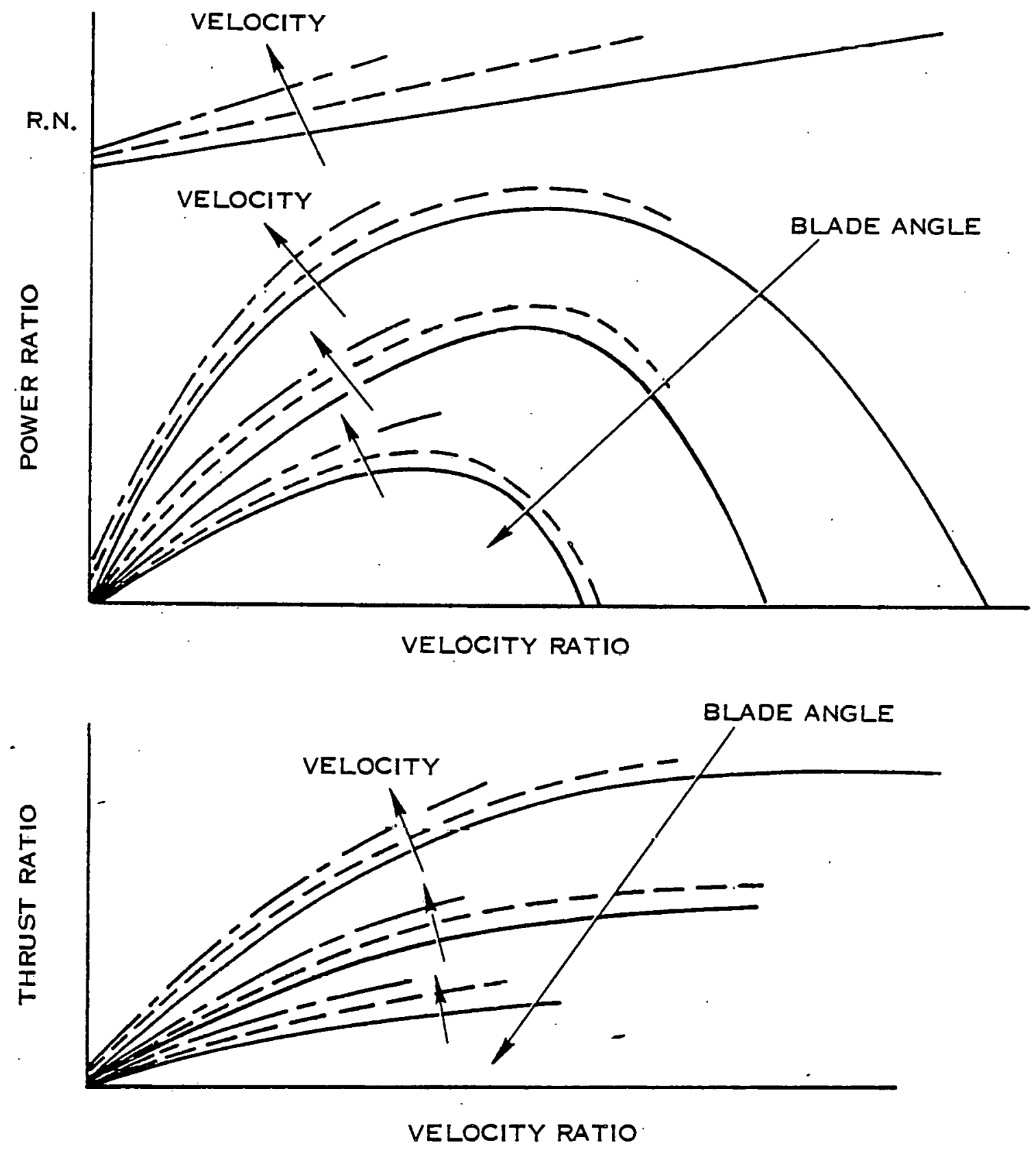

FIGURE A-3 TYPICAL DATA PRESENTATION PLOTS 


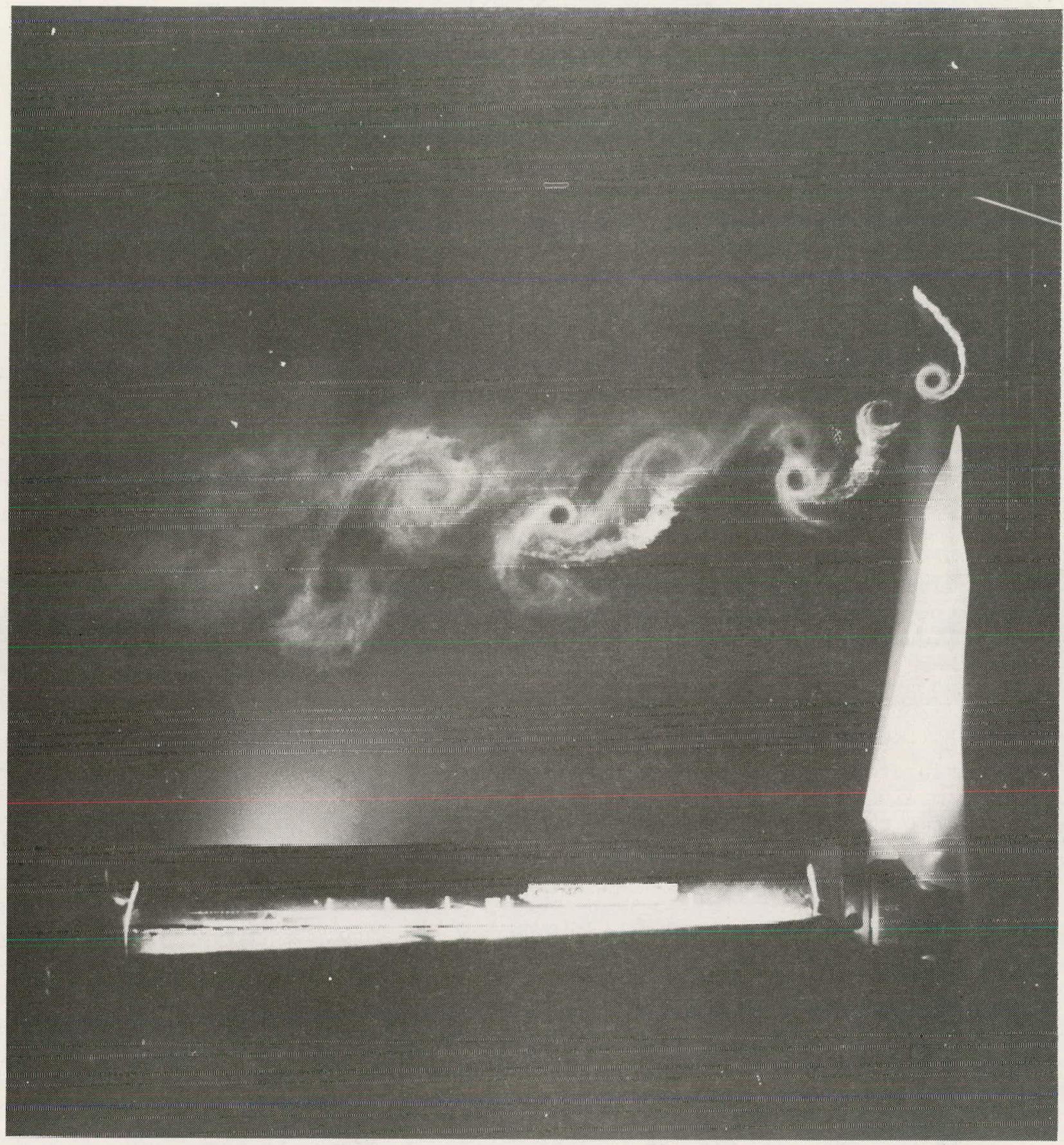

1

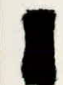

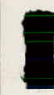

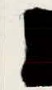

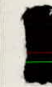

1

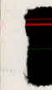

1

1

1

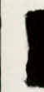

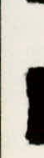

FIGURE A-4. FLOW VISUALIZATION TIP VORTEX 


\title{
APPENDIX B
}

\author{
INVESTIGATION OF THE SEPARATION OF A BLADE \\ FROM A WIND TURBINE MODEL DURING \\ WIND TUNNEL TESTING
}

\section{B1 BACKGROUND}

A test program on a series of wind turbine models was in progress at the United Technologies Research Center wind tunnel when on the night of July 27, 1976, a blade separated from one of the models and the test pod separated from the pylon which supported the pod and turbine on the tunnel balance system.

The overall test program included four types of turbine blade configurations; 15 Activity Factor (AF), $30 \mathrm{AF}$, and $60 \mathrm{AF}$ with $230 \mathrm{XX}$ airfoils and $60 \mathrm{AF}$ with $44 \mathrm{XX}$ airfoils. The 15 and $30 \mathrm{AF}$ blades were manufactured from solid aluminum. The two sets of $60 \mathrm{AF}$ blades were fabricated with a part span aluminum spar which included the blade retention area and to which was attached a laminated wood structure which formed the entire airfoil section of the blade. The $15 \mathrm{AF}$ blades were tested in a twobladed turbine and the $30 \mathrm{AF}$ blades were tested in both two and three bladed turbines. During the initial testing of the first of the $60 \mathrm{AF}$ blade designs (Fig. B-1) the wood section of one of the blades separated from the aluminum spar and the resulting turbine unbalance caused the pod containing the gearbox and generator as well as the hub and remaining blade to separate from the support pylon (Fig. B-2).

\section{B2 EVENTS PRECEDING INCIDENT}

On the initial run-up of the two-bladed $60 \mathrm{AF}$ turbine excessive rig vibratory amplitude was noted from the rig vibration pickup in the 900 to 1100 RPM range. The rig was shut down for examination which revealed that the two blade tips differed in track by approximately .13m (.5") and the two blades were at different angles. The turbine was removed from the rig to check static mass balance which was satisfactory. The turbine was reinstalled on the rig and the blades adjusted to the same angle ( 1.5 deg.). On the subsequent run-up the peak rig motion was noted at 1100 RPM and, although higher than encountered with the previous models, was considered acceptable at $\pm .004 \mathrm{~m}\left(.015^{\prime \prime}\right)$. At the maximum test RPM of 1671 the motion was \pm .002 (.0065").

The test procedure used was the same as that with the previous models. The turbine was powered up to the maximum design tip speed of $213 \mathrm{~m} / \mathrm{s}$ (700 fps) at 1671 RPM. The tunnel air speed was then brought up to the test value of $13 \mathrm{~m} / \mathrm{s}$ ( $44 \mathrm{fps}$ ) at which time the drive motor has converted to generator and is absorbing power. Data was obtained at the first test point. The load was then increased to reduce RPM to 1540 and data obtained at that point. While changing to the next point, $1433 \mathrm{RPM}$, and without any other indication, a thump was heard and it was noted that the test rig had disappeared from the observation window. At this point the turbine was producing approximately $1.5 \mathrm{KW}$ (2 HP) with $445 \mathrm{~N}$ (100 1bs.) of drag which is well below the design values of $54 \mathrm{KW}$ (73 HP) and $2362 \mathrm{~N}$ (531 lbs.) of drag. 


\section{B3 INVESTIGATION}

Inspection in the tunnel revealed that the test pod and turbine had separated at the top of the pylon and was hanging by the instrumentation and lubrication lines. One blade was still in the hub and was wedged under the trolley rail. The other blade spar was still in the hub with no wood structure attached. Various size pieces of the separated wood structure were scattered in the tunnel (Fig. B-3, B-4).

Subsequent inspection indicated that the aluminum plate attaching the pod to the pylon had fractured in three places due to severe overload. There were no indications of fatigue damage.

Examination of the separated blade and associated hardware as we11 as the other previously tested blades revealed several items which may have been contributory to the separation. These were:

1) There appeared to be a poor band between the aluminum spar and the laminated wood structure.

2) There was incomplete engagement in the wood of an aluminum pin which provided a redundant retention of the wood to the spar.

3) Masking tape had been used to hold the wood laminate butt joints in line during the assembly and bonding process which resulted in bands of unbonded surfaces in the completed assembly. Unfortunately, a series of these bands passed directly through the position of the redundant pin resulting in reduced strength at that point.

4) Markings on the blade retention fillet area indicated that there was not a good fit between the blade and hub or that the blade was not fully seated in the hub under operating conditions. Additional markings on the shank at the position of the hub outside diameter indicated that in-plane blade vibration had occurred of sufficient amplitude to result in contact with the hub.

It was concluded that the separation started at the spar bond interface which transfer the load to the pin. Because of the unbonded areas near the pin and the incomplete engagement of the pin, the laminates separated in shear tearing out a section of wood and releasing the remaining wood structure.

The markings on the blade shanks cause a suspicion that an unanticipated blade vibration condition, possibly involving a blade/rig interaction, may have been encountered which could have increased the total loadings on the wood to spar interfaces.

\section{B4 RECOMMENDATIONS}

Before additional testing is done the blades should be reworked or rebuilt to eliminate the noted discrepancies, particularly with respect to the spar bond and the location of the pin with respect to the laminate butt joints. In addition, future running should be done with strain gages installed on the blades to permit stress and frequency monitoring. Also, the rig vibration pickup readouts should be modified to permit indication of frequency. 
MODEL NO. 5

60 ACTIVITY FACTOR

NACA $230 \times X$ AIRFOIL SECTIONS

2. $4 \mathrm{M}$ (8.0FT) DIAMETER

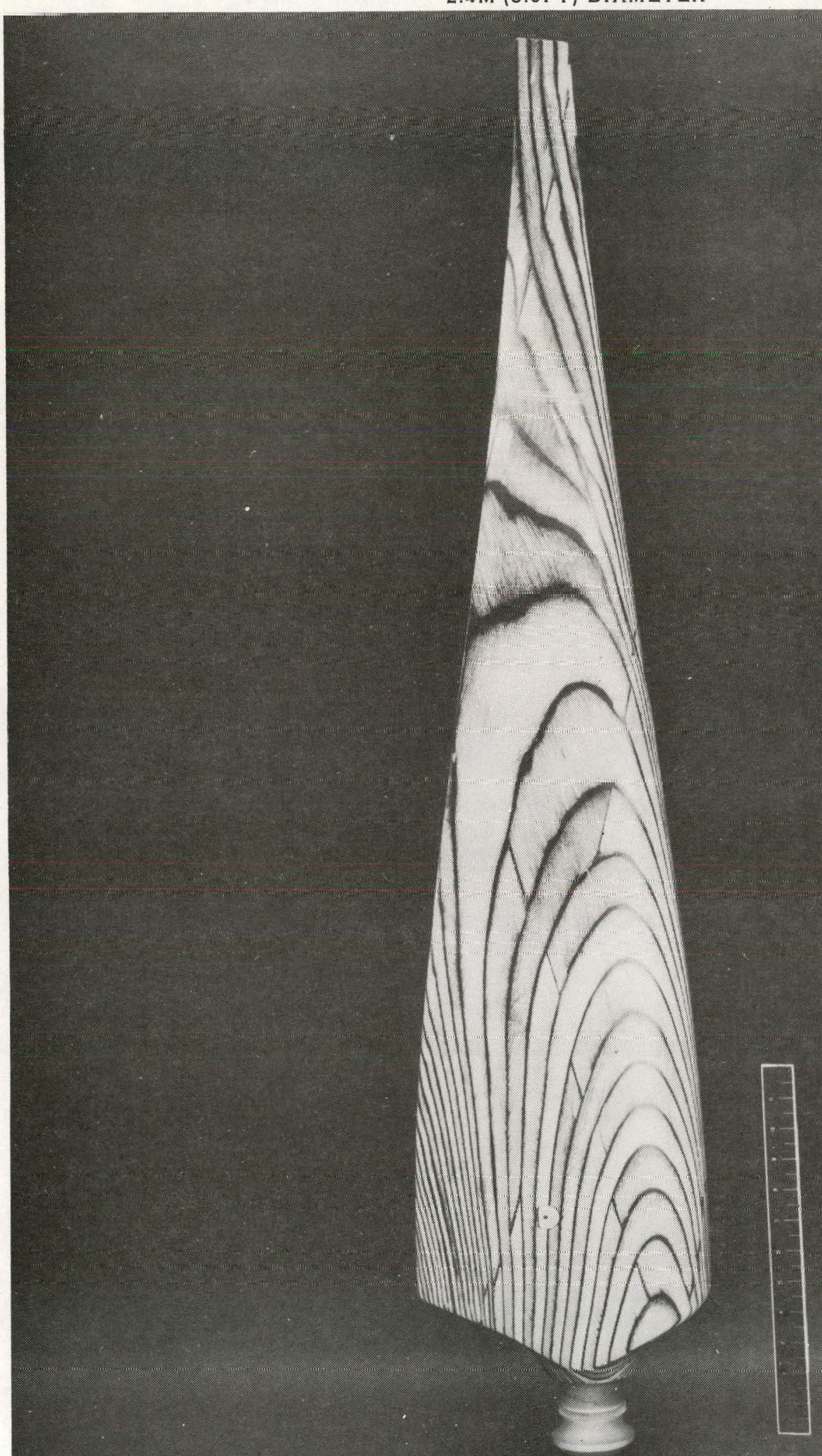

FIGURE B-1. BLADE SHAPE 


\section{$-\infty-\infty-\infty-\infty-\infty-\infty-\infty, \infty$}

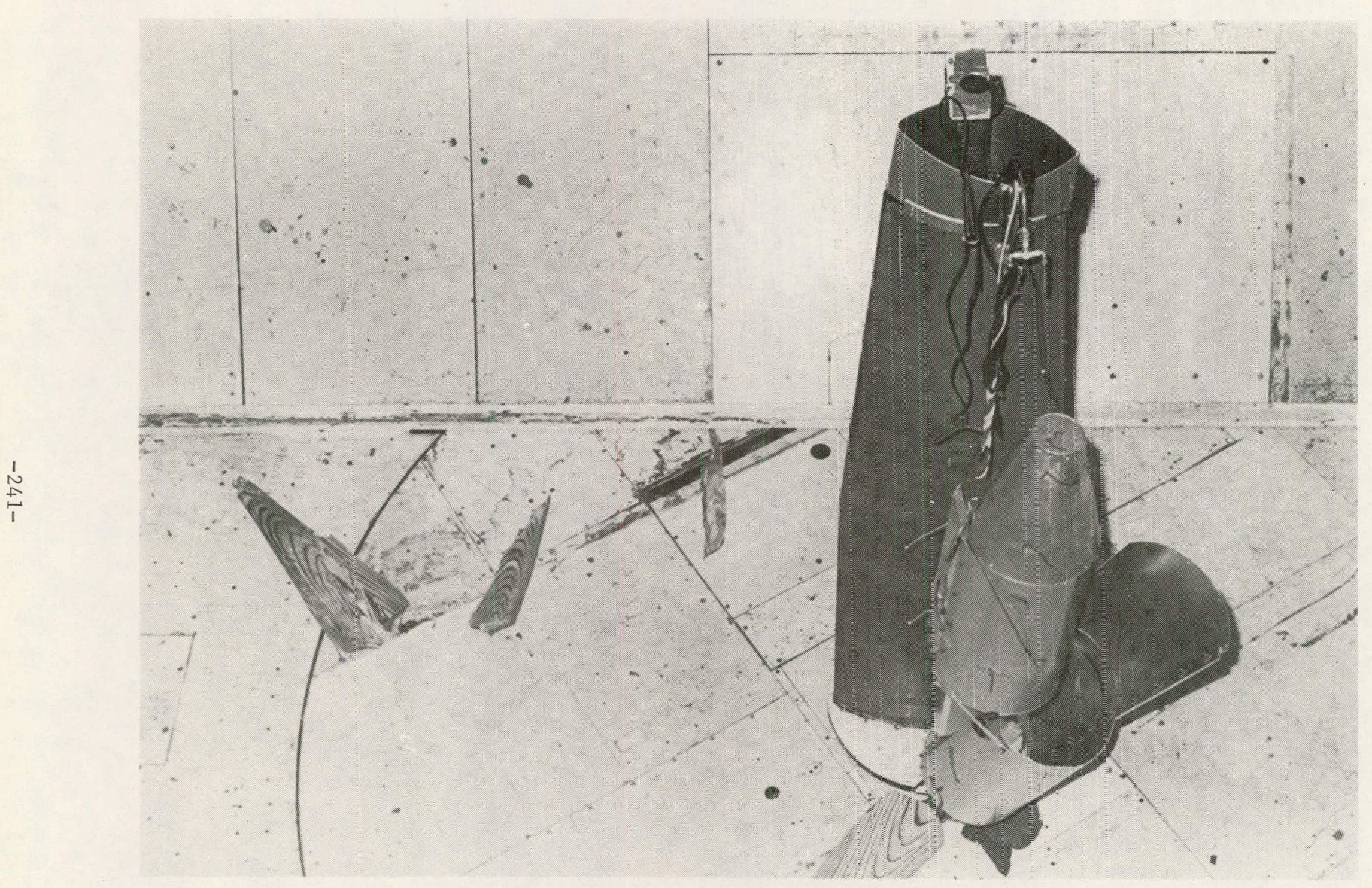

FIGURE B-2. FAILED TURBINE RIG 


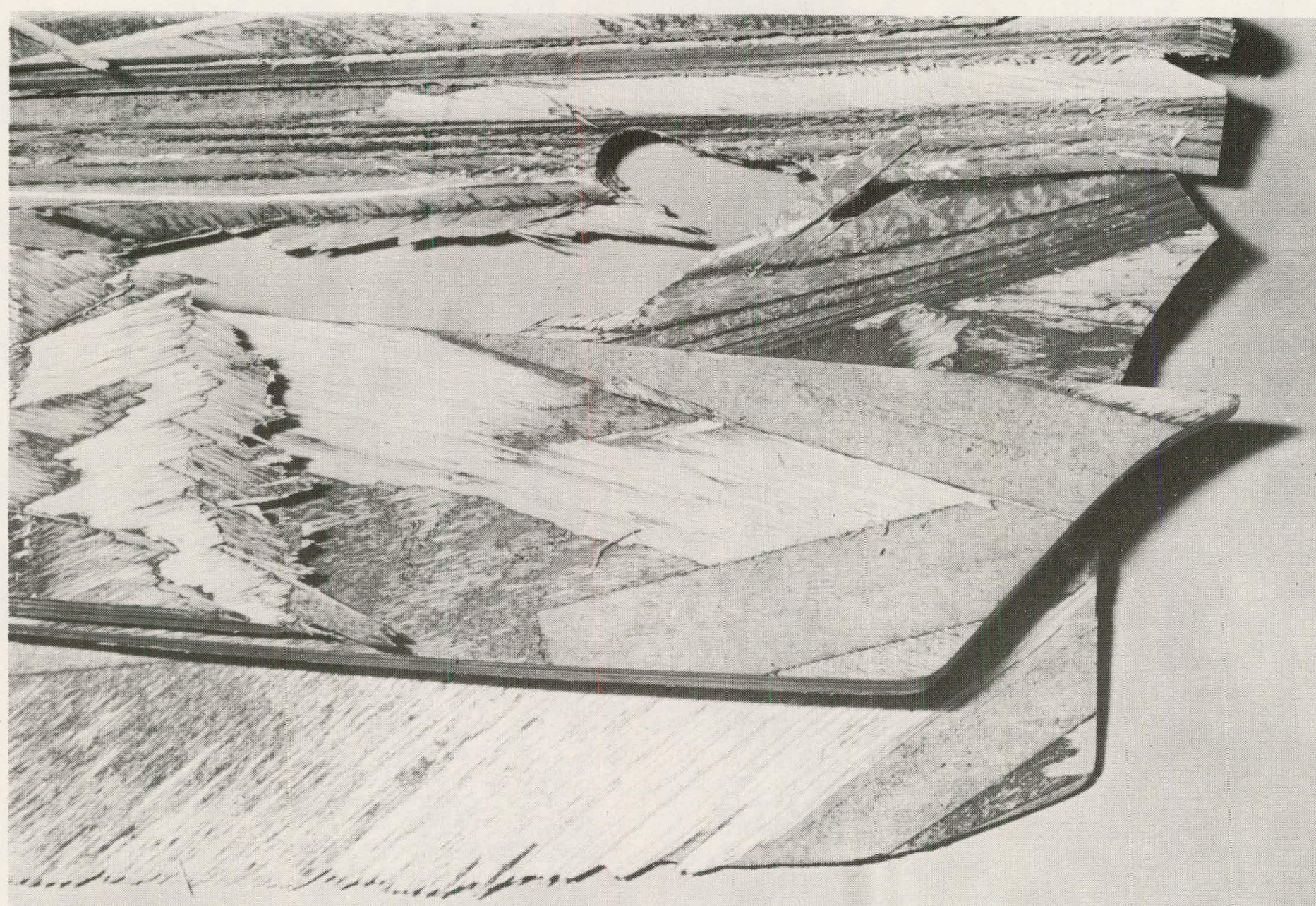




\section{APPENDIX C}

\section{C1 DATA REDUCTION EQUATIONS}

All of the measurements obtais ! during the course of the testing were recorded on magnetic tape. A computer prugram for rapid reduction of these data was developed. The equations used in this computer program are given in this section.

\section{C1. 1 Tunnel Flow Conditions}

$$
\begin{aligned}
& \mathrm{P}_{\mathrm{TO}}=\mathrm{H} \\
& \mathrm{P}_{0 \mathrm{u}}=\mathrm{H}+\left(\mathrm{P}_{0 \mathrm{u}}-\mathrm{H}\right) \\
& \mathrm{T}_{\mathrm{T} 0 \mathrm{RAN}}=\mathrm{T}_{\mathrm{T} 0}+459.7 \\
& \mathrm{M}_{\mathrm{u}}=\left[5+\left(\frac{\mathrm{P}_{\mathrm{TO}}}{\mathrm{P}_{0 \mathrm{u}}}\right)^{\frac{\gamma-1}{}}-5\right]^{1 / 2} \\
& \mathrm{~T}_{\mathrm{Ou}}=\left[1+\left(\frac{\gamma-1}{2}\right) \mathrm{M}_{\mathrm{u}}^{2}\right]^{-1} \mathrm{~T}_{\mathrm{T} 0 \mathrm{RAN}} \\
& a_{u}^{\prime}=\left[g R^{\prime} T_{0 u}\right]^{1 / 2} \\
& v_{u}=a_{u}^{\prime} M_{u} \\
& \rho=\left[1+\left(\frac{\gamma-1}{2}\right) \mathrm{M}_{\mathrm{u}}^{2}\right]-\left(\frac{1}{\gamma-1}\right) \rho_{0} \\
& \boldsymbol{\nu}=1.36 \times 10^{2}\left[\left(\frac{\mathrm{T}_{\mathrm{T} 0 \mathrm{RAN}}}{510}\right)^{5}\right]^{1 / 2}\left[\left(\mathrm{P}_{\mathrm{T} 0} \times 144\right)\left(0.555 \mathrm{~T}_{\mathrm{T} 0 \mathrm{RAN}}+120\right)\right]^{-1} \\
& \mathrm{R}_{\mathrm{Nu}}=\mathrm{XL}\left[\left(\frac{2 \mathrm{~T}_{1} \mathrm{~N} \mathrm{R}_{0}}{60}\right)^{2}+\left(\mathrm{v}_{\mathrm{u}} \cos \psi\right)^{2}\right]^{1 / 2} \nu^{-1} \\
& \mathrm{Q}_{0 \mathrm{u}}=\frac{\rho \mathrm{V}_{\mathrm{u}}^{2}}{2}
\end{aligned}
$$




\section{C1. 2 Model Load Conditions}

$$
\tau=[\text { CHANNEL } 1(\mathrm{R})-\text { CHANNEL } 1(0)] \mathrm{K}_{1}-\mathrm{T}_{\mathrm{TARE}}
$$

Correcting Balance Readings for Start Zeros

$$
\begin{aligned}
& \mathrm{L}^{\prime}=\mathrm{L}_{\mathrm{R}}-\mathrm{L}_{0} \\
& \mathrm{D}^{\prime}=\mathrm{D}_{\mathrm{R}}-\mathrm{D}_{0} \\
& \mathrm{P}_{\mathrm{MBRC}}^{\prime}=\mathrm{P}_{\mathrm{MR}}-\mathrm{P}_{\mathrm{M} 0} \\
& \mathrm{Y}_{\mathrm{MBRC}}^{\prime}=\mathrm{Y}_{\mathrm{MR}}-\mathrm{Y}_{\mathrm{M} 0} \\
& \mathrm{R}_{\mathrm{MBRC}}^{\prime}=\mathrm{R}_{\mathrm{MR}}-\mathrm{R}_{\mathrm{M} 0} \\
& \mathrm{Y}_{\mathrm{BRC}}^{\prime}=\mathrm{Y}_{\mathrm{R}}-\mathrm{Y}_{0}
\end{aligned}
$$

Transfer of Moments from the Balance to the Model Resolving Center

$$
\begin{aligned}
& \mathrm{X}=\left[\mathrm{X}_{1}+\left(\mathrm{X}_{0}^{2}+\mathrm{Z}_{0}{ }^{2}\right)^{1 / 2} \cos (\phi-\mathrm{a})\right] \cos \psi+\mathrm{Y}_{0} \sin \psi \\
& \mathrm{Y}=-\left[\mathrm{X}_{1}+\left(\mathrm{X}_{0}^{2}+\mathrm{Z}_{0}^{2}\right)^{1 / 2} \cos (\varphi-\mathrm{a})\right] \sin \psi+\mathrm{Y}_{0} \cos \psi \\
& \mathrm{Z}=\mathrm{Z}+\left(\mathrm{X}_{0}^{2}+\mathrm{Z}_{0}{ }^{2}\right)^{1 / 2} \sin (\phi-\mathrm{a}) \\
& \mathrm{P}_{\mathrm{MMRC}}^{\prime}=\mathrm{P}_{\mathrm{MBRC}}^{\prime}+\mathrm{L}_{\mathrm{X}}^{\prime} \mathrm{X}-\mathrm{D}_{\mathrm{Z}}^{\prime} \\
& \mathrm{Y}_{\mathrm{MBRC}}^{\prime}=\mathrm{Y}_{\mathrm{MBRC}}^{\prime}+\mathrm{Y}_{\mathrm{X}}^{\prime}-\mathrm{D}_{\mathrm{Y}}^{\prime} \\
& \mathrm{R}_{\text {MMRC }}^{\prime}=\mathrm{R}_{\mathrm{MBRC}}^{\prime}+\mathrm{L}_{\mathrm{Y}}^{\prime}-\mathrm{Y}_{\mathrm{Z}}^{\prime}
\end{aligned}
$$$$
\text { Aerodynamic Tare Corrections }
$$$$
L=L^{\prime}-L_{T} Q_{0 u}
$$$$
\mathrm{D}=\mathrm{D}^{\prime}-\mathrm{D}_{\mathrm{T}} \mathrm{Q}_{0 \mathrm{u}}
$$$$
\text { where: } \mathrm{L}_{\mathrm{T}}=\mathrm{P}_{\mathrm{MT}}=\mathrm{Y}_{\mathrm{MT}}=\mathrm{R}_{\mathrm{MT}}=0.0
$$$$
P_{M}=P^{\prime} \text { MMRC }-P_{M T} Q_{0 u}^{\prime}
$$$$
\mathrm{Y}_{\mathrm{M}}=\mathrm{Y}_{\mathrm{MMRC}}^{\prime}-\mathrm{Y}_{\mathrm{MT}} \mathrm{Q}_{\mathrm{Ou}}
$$$$
\mathrm{R}_{\mathrm{M}}=\mathrm{R}_{\mathrm{MMRC}}^{\prime}-\mathrm{R}_{\dot{\mathrm{MT}}} \mathrm{Q}_{\mathrm{Ou}}
$$$$
\mathrm{Y}=\mathrm{Y}^{\prime}-\mathrm{Y}_{\mathrm{T}} \mathrm{Q}_{\mathrm{Ou}}
$$ 
Conversion of Forces and Moments to Parameter Form

$$
\begin{aligned}
& \mathrm{TR}=\frac{-\mathrm{D}}{1 / 2 \rho \mathrm{R}^{2} \pi \mathrm{V}_{0}^{2}}=\text { THRUST RATIO } \\
& \mathrm{NR}=\frac{\mathrm{Y}}{1 / 2 \rho \mathrm{R}^{2} \pi \mathrm{V}_{0}^{2}}=\text { INPLANE VERTICAL FORCE COEFF. } \\
& \mathrm{SR}=\frac{\mathrm{L}}{1 / 2 \rho \mathrm{R}^{2} \pi \mathrm{V}_{0}^{2}}=\text { INPLANE HORIZONTAL FORCE COEFF } \\
& \mathrm{MR}=\frac{-\mathrm{Y}_{\mathrm{M}}}{1 / 2 \rho \mathrm{R}^{3} \pi \mathrm{V}_{0}^{2}}=\text { HORIZONTAL PLANE MOMENT COEFF. } \\
& \mathrm{YR}=\frac{\mathrm{P}_{\mathrm{M}}}{1 / 2 \rho \mathrm{R}^{3} \pi \mathrm{V}_{0}^{2}}=\text { VERTICAL PLANE MOMENT COEFF. } \\
& \mathrm{VR}=\frac{2{ }_{1} \mathrm{~N} R}{60 \mathrm{~V}_{0}}=\text { VELOCITY RATIO } \\
& \mathrm{PR}=\frac{\tau 2 \pi \mathrm{N}}{1 / 2 \rho \mathrm{R}^{2} \pi \mathrm{V}_{0}^{3} \times 60}=\text { POWER RATIO }
\end{aligned}
$$

\section{C1. 3 Wake and Solid Body Blockage Corrections}

a) Solid Body Blockage

1. Support Strut Fairing

$$
\begin{aligned}
& \epsilon_{\mathrm{SB}}=\frac{\mathrm{k}_{1} \tau_{1} \text { (Volume) }}{\mathrm{S} 3 / 2} \\
& \mathrm{k}_{1}=1.0 ; \tau_{1}=0.9 ; \text { Volume }=6.3 ; \mathrm{S}=267 . \\
& \epsilon_{\mathrm{SB}_{\mathrm{F}}}=0.0013
\end{aligned}
$$


2. Drive System Pylon

$$
\begin{aligned}
& \epsilon_{\mathrm{SB}}=\frac{\mathrm{k}_{3} \tau_{1} \text { (Volume) }}{\mathrm{S} / 2} \\
& \mathrm{k}_{3}=0.96 ; \tau_{1}=0.8 ; \text { Volume }=9.0 ; \mathrm{S}=267 \\
& \epsilon_{\mathrm{SB} \cdot \mathrm{P}}=0.003
\end{aligned}
$$

3. Total Solid Body Blockage

$$
\begin{aligned}
\epsilon_{\mathrm{SB}} & =\epsilon_{\mathrm{SB}_{\mathrm{F}}}+\epsilon_{\mathrm{SB}} \\
& =0.0043
\end{aligned}
$$

b) Turbine Wake Blockage

$$
\epsilon_{\mathrm{WB}}=\frac{\mathrm{AC}_{\mathrm{D}}}{4 \mathrm{~S}}
$$

where,

$$
C_{D}=\frac{D}{Q A}
$$

hence,

$$
\epsilon_{\mathrm{WB}}=\frac{\mathrm{D}}{4 \mathrm{SQ} \mathrm{Qu}_{\mathrm{u}}}
$$

or

$$
\epsilon_{\mathrm{WB}}=\frac{\mathrm{T}_{\mathrm{R} A \mathrm{Q}_{0 u}}}{4 \mathrm{SQ} \mathrm{Q}_{\mathrm{u}}}
$$

thus,

$$
\epsilon_{\mathrm{WB}}=0.04707 \mathrm{~T}_{\mathrm{R}}
$$

c) Support System Wake Blockage

1. Support Strut Fairing

$$
\begin{aligned}
& \epsilon_{\mathrm{WB}_{F}}=\frac{A \mathrm{C}_{\mathrm{D}}}{4 \mathrm{~S}} \\
& \mathrm{~A}=18 ; \mathrm{C}_{\mathrm{D}}=0.01 ; \mathrm{S}=267 \\
& \epsilon_{\mathrm{WB}_{\mathrm{F}}}=0.00017
\end{aligned}
$$


d) Total Blockage Correction

$$
\begin{aligned}
& \epsilon=\Sigma \epsilon_{\mathrm{SB}}+\Sigma \epsilon_{\mathrm{WB}} \\
& \mathrm{V}_{0}=\mathrm{v}_{0 \mathrm{u}}[1+\epsilon]
\end{aligned}
$$

or

$$
\mathrm{V}_{0}=\mathrm{V}_{0 \mathrm{u}}\left[1+0.00485+.0 .04707 \mathrm{~T}_{\mathrm{R}}\right]
$$


C1.4 SYMBOLS FOR DATA REDUCTION EQUATIONS

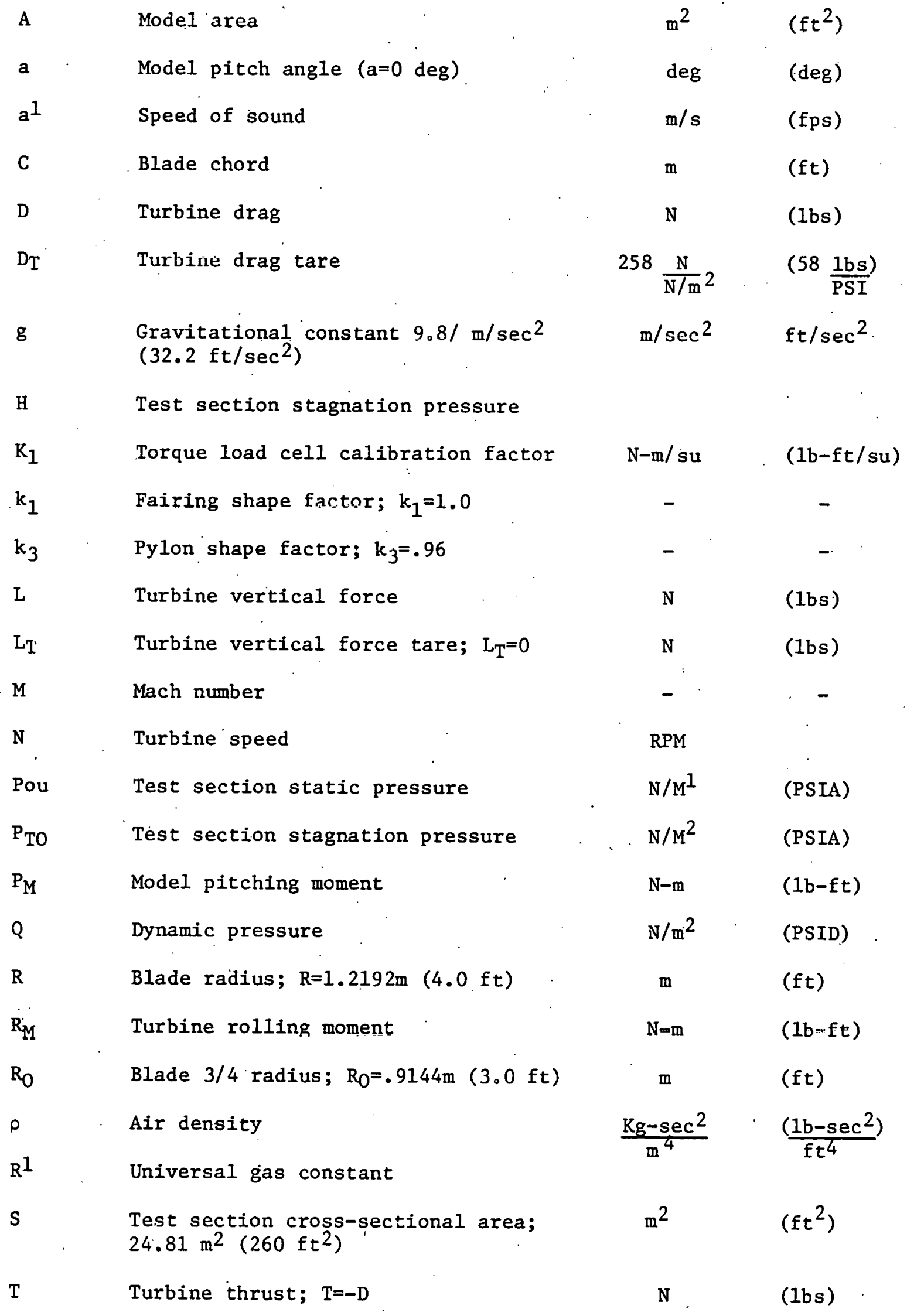




\begin{tabular}{|c|c|c|c|c|}
\hline $\mathrm{T}_{\mathrm{TO}}$ & Test section stagnation temperature & ${ }^{\circ} \mathrm{C}$ & & $\left(O^{\circ}\right)$ \\
\hline $\mathrm{v}_{0}$ & Tunnel velocity corrected for blockage & $\mathrm{m} / \mathrm{sec}$ & & $(\mathrm{ft} / \mathrm{sec})$ \\
\hline Vou & Tunnel velocity uncorrected & $\mathrm{m} / \mathrm{sec}$ & & $(\mathrm{ft} / \mathrm{sec})$ \\
\hline $\mathrm{x}$ & $\begin{array}{l}\text { Longitudinal distance from the } \\
\text { balance to the model resolving center }\end{array}$ & m & & $(f t)$ \\
\hline$x_{0}$ & $\begin{array}{l}\text { Longitudinal distance from axis of pitch } \\
\text { motion to model resolving center, at } \\
\text { zero pitch and yaw }\end{array}$ & m & & $(f t)$ \\
\hline$x_{1}$ & $\begin{array}{l}\text { Longitudinal distance, at zero yaw and } \\
\text { pitch, from balance resolving center } \\
\text { to axis of pitch motion; } x_{1}=0\end{array}$ & $\mathbf{m}$ & & $(f t)$ \\
\hline $\mathrm{XL}$ & Reference length (Chord at $3 / 4$ span) & $\mathfrak{m}$ & & $(f t)$ \\
\hline $\mathrm{Y}$ & Horizontal lateral in-plane force & $\mathbf{N}$ & & (1bs) \\
\hline $\mathrm{y}$ & $\begin{array}{l}\text { Lateral distance from the balance to } \\
\text { the model resolving center; } y=0.0\end{array}$ & m & & $(f t)$ \\
\hline$y_{0}$ & $\begin{array}{l}\text { Lateral distance, at zero pitch and } \\
\text { yaw, from the axis of yaw motion to } \\
\text { the model resolving center }\end{array}$ & m & & $(f t)$ \\
\hline $\mathrm{Y}_{\mathrm{M}}$ & Yawing moment & $\mathrm{N}-\mathrm{m}$ & $\vdots$ & $(1 b-f t)$ \\
\hline $\mathrm{z}$ & $\begin{array}{l}\text { Vertical distance from the Model to } \\
\text { the balance resolving center; } \quad \mathrm{Z}=0\end{array}$ & $\mathbf{m}$ & & $(f t)$ \\
\hline $\mathbf{z}$ & $\begin{array}{l}\text { Vertical distance, at zero yaw and } \\
\text { pitch, from the balance resolving } \\
\text { center to the axis of pitch motion; } \\
z=0.0\end{array}$ & $\mathrm{~m}$ & & $(f t)$ \\
\hline$\beta$ & Turbine blade angle & degs & & - \\
\hline$\gamma$ & Ratio of specific heats, 1.4 & & & \\
\hline$\theta$ & $\operatorname{Arctan} z / X_{0}$ & degs & & - \\
\hline$\Psi$ & Wind inflow angle & degs & & - \\
\hline \multicolumn{2}{|c|}{ Subscripts } & & & . \\
\hline $\mathrm{u}$ & $\begin{array}{l}\text { Uncorrected for solid body and wake } \\
\text { blockage }\end{array}$ & & & \\
\hline o & Stagnation conditions & & & \\
\hline
\end{tabular}

